\title{
Optical Properties of a Five-layer Slab with Long-period Gratings
}

by

\author{
Alexander Andreyuk \\ A thesis submitted to the \\ Faculty of Graduate Studies and Research \\ in partial fulfillment of the requirements for the degree of \\ Master of Applied Science \\ In Electrical Engineering
}
Ottawa-Carleton Institute for Electrical Engineering
Department of Electronics
Faculty of Engineering
Carleton University
Ottawa, Canada

(C) Alexander Andreyuk, 2006 


$\begin{array}{ll}\begin{array}{l}\text { Library and } \\ \text { Archives Canada }\end{array} & \begin{array}{l}\text { Bibliothèque et } \\ \text { Archives Canada }\end{array} \\ \begin{array}{l}\text { Published Heritage } \\ \text { Branch }\end{array} & \begin{array}{l}\text { Direction du } \\ \text { Patrimoine de l'édition }\end{array} \\ \begin{array}{l}\text { 395 Wellington Street } \\ \text { Ottawa ON K1A ON4 }\end{array} & \begin{array}{l}\text { 395, rue Wellington } \\ \text { Ottawa ON K1A ON4 } \\ \text { Canada }\end{array}\end{array}$

Your file Votre référence ISBN: 978-0-494-16451-8 Our file Notre référence ISBN: 978-0-494-16451-8

NOTICE:

The author has granted a nonexclusive license allowing Library and Archives Canada to reproduce, publish, archive, preserve, conserve, communicate to the public by telecommunication or on the Internet, loan, distribute and sell theses worldwide, for commercial or noncommercial purposes, in microform, paper, electronic and/or any other formats.

The author retains copyright ownership and moral rights in this thesis. Neither the thesis nor substantial extracts from it may be printed or otherwise reproduced without the author's permission.
AVIS:

L'auteur a accordé une licence non exclusive permettant à la Bibliothèque et Archives Canada de reproduire, publier, archiver, sauvegarder, conserver, transmettre au public par télécommunication ou par l'Internet, prêter, distribuer et vendre des thèses partout dans le monde, à des fins commerciales ou autres, sur support microforme, papier, électronique et/ou autres formats.

L'auteur conserve la propriété du droit d'auteur et des droits moraux qui protège cette thèse. $\mathrm{Ni}$ la thèse ni des extraits substantiels de celle-ci ne doivent être imprimés ou autrement reproduits sans son autorisation.
In compliance with the Canadian

Privacy Act some supporting forms may have been removed from this thesis.

While these forms may be included in the document page count, their removal does not represent any loss of content from the thesis.
Conformément à la loi canadienne sur la protection de la vie privée, quelques formulaires secondaires ont été enlevés de cette thèse.

Bien que ces formulaires aient inclus dans la pagination, il n'y aura aucun contenu manquant.

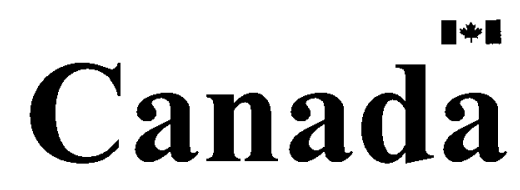




\begin{abstract}
In recent years optical devices based on fiber gratings have been widely used in telecommunication systems as well as in various precision measurements as sensing elements. They prove to be effective, robust and low cost solutions. The subject of this thesis is the design and analysis of one of such device - a planar waveguide structure with a long period grating that is sensitive to a small refractive index variation and therefore suitable for all optical switching system.

The two-channel waveguide is investigated theoretically with perturbation theory. We have found an approximate closed form expression for modal indices in that structure. This analytical solution is compared with numerical simulations from a commercial simulation tool, and we find a reasonably good agreement. Then we introduce a long period grating in our mathematical model and evaluate its spectral transmission characteristics. Under certain geometrical and material parameters, the transmission characteristics exhibit dual peak resonance, i.e. a phase matching condition is satisfied at two distinct wavelengths. By optimizing the above parameters, the dual peak resonance can be made very sensitive to small internal refractive index changes. Our numerical simulations show that the transmission spectrum of the five-layer slab with a long period grating exhibits a wavelength shift for the loss peak $\Delta \lambda=8 \mathrm{~nm}$ for a refractive index change of $\Delta n=10^{-6}$. Such a high sensitivity makes it attractive for use in all optical switching systems. We discuss possible applications and the effect of parameters fluctuations and physical constrains.
\end{abstract}




\section{Acknowledgements}

I would like to thank my supervisors professor Jacques Albert and professor Alan Steele who encourage and support me. Their regular discussions of that project were very helpful and exciting. I am grateful and deeply indebted for their patience and professional guidance throughout this research.

I also want to thank the Department of Electronics for financial support and all professors whose courses I had a chance to take. 


\section{Table of Contents}

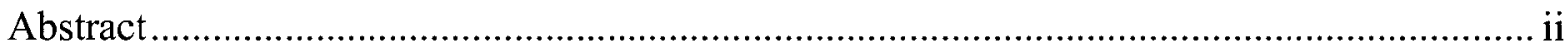

Acknowledgements..................................................................................................... ii

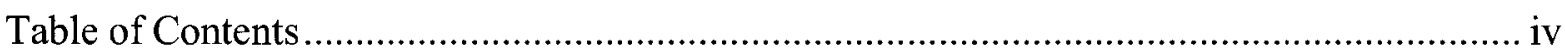

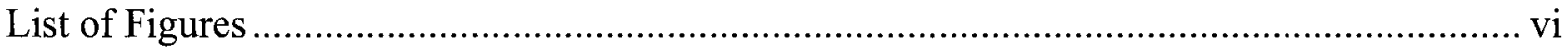

List of Symbols and Acronyms................................................................................. xiii

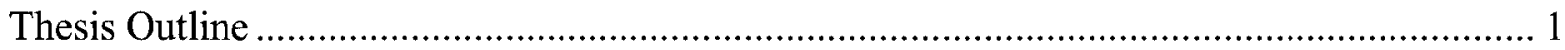

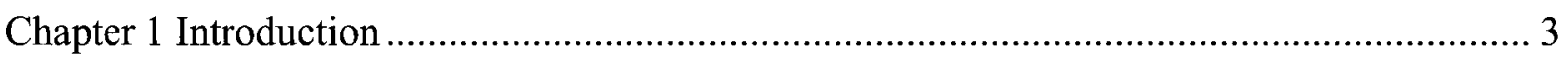

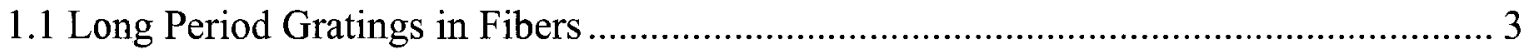

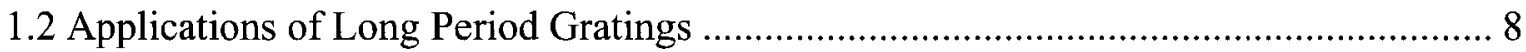

1.2.1 Rejection filter, bandpass filter, tunable filter. [1] - [4] ………………………... 8

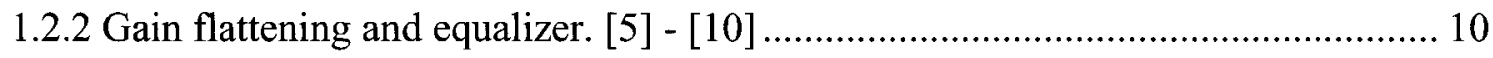

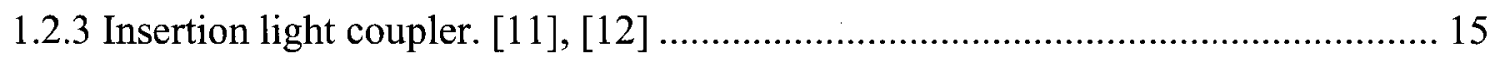

1.2.4 Dispersion compensation. [13] .................................................................. 17

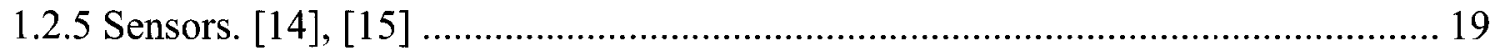

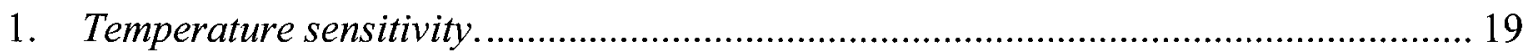

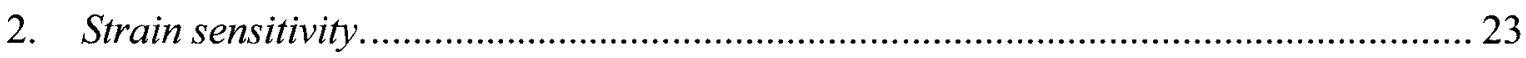

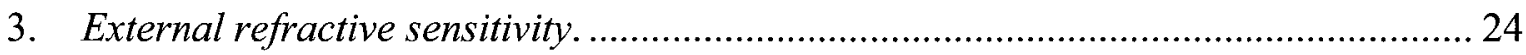

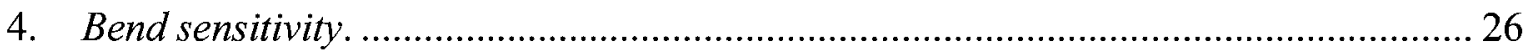

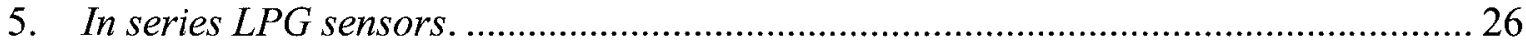

1.3 Long period gratings in planar waveguides. [17]-[22]............................................. 28

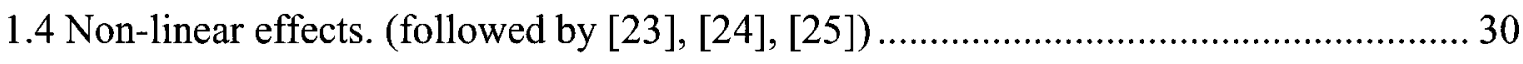

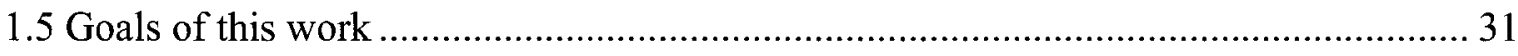

Chapter 2 Coupled mode theory and its applications ............................................................ 33

2.1 Coupled mode theory (CMT) ............................................................................... 33

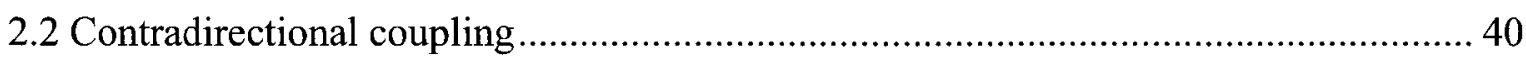

2.2.1 Simple solutions of coupled equations .................................................................. 42

2.2.2 Transfer matrix for FBG................................................................... 44

2.2.3 Analytical solution for non-uniform gratings ........................................................ 48 


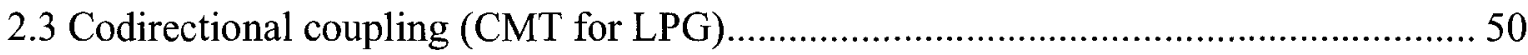

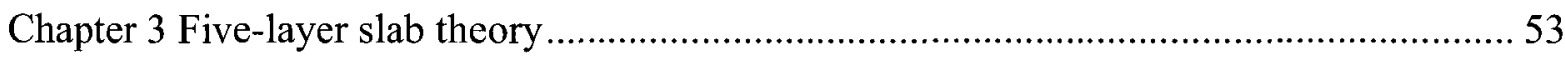

3.1 Wave equation for dielectric planar waveguide ........................................................... 54

3.2 Three-layer symmetric slab waveguide. [32], [33] …….......................................... 58

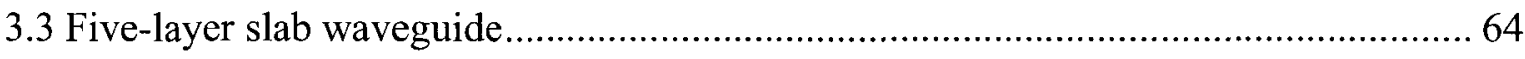

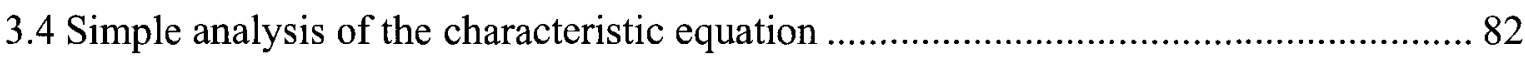

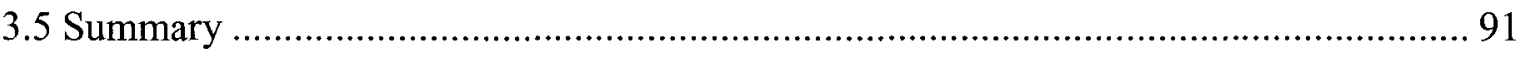

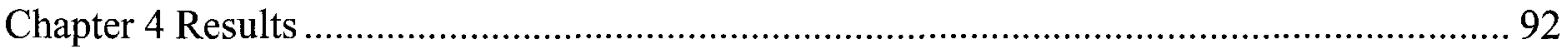

4.1 Approximate analytical modal indices for five-layer slab .......................................... 94

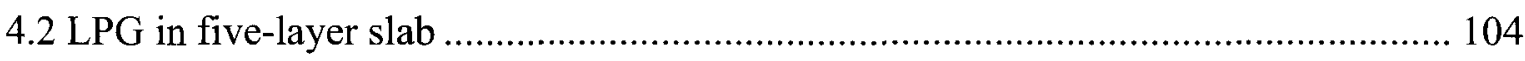

4.2.1 Influence of geometry and material choice ……………………….................. 120

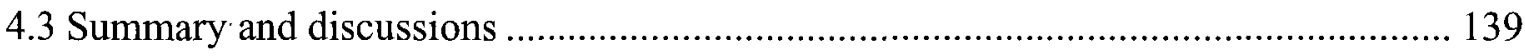

Chapter 5 Discussion and Conclusions...................................................................... 142

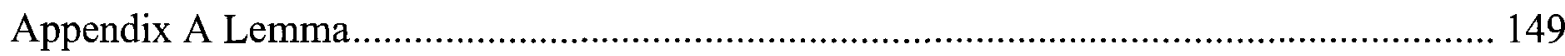

Appendix B Perturbation theory for stationary states. (Rayleigh- Schrödinger perturbation

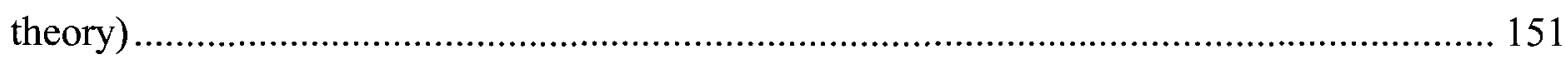

Appendix C Characteristic equation for symmetric three-layer slab waveguide ….............. 156

Appendix D Propagation constant in symmetric three-layer slab waveguide ....................... 159

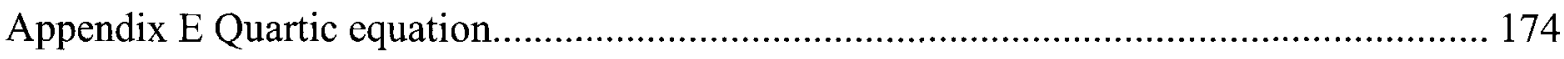

Appendix F Optical Kerr effect. (followed by [23], [24], [25]) …………........................ 177

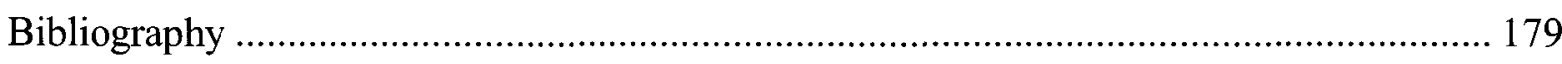




\section{List of Figures}

Fig. 1-1. Scattering off LPG in fiber. Momentum conservation in photon picture .............. 5

Fig. 1-2a. Transmission characteristic UV-induced LPG in fiber. [1] ............................ 6

Fig. 1-2b. Transmission characteristic LPG fabricated by periodic deformation of the core of the fiber using $\mathrm{CO}_{2}$ laser. [2] ....................................................................... 7

Fig. 1-3a. Band-pass filter scheme using two long period gratings in series. The core mode blocker prevents light from passing through the core. The cladding mode modulator controls the transmission through the device. [3]

Fig. 1-3b. Measured transmission spectrum of bandpass filter showing the main passband channel at $\sim 1527 \mathrm{~nm}$ and adjacent channels from different cladding modes. The insert shows that the detailed spectrum of the central peak, which has a 2-nm bandwidth at -3 dB. [3] 10

Fig. 1-4. energy levels of $\mathrm{Er}^{3+}$ ions used in EDFA. Suitable pumps are at $\sim 980 \mathrm{~nm}$ and $\sim 1480 \mathrm{~nm} . \tau_{21} \gg \tau_{32}, \tau_{21} \sim 10 \mathrm{~ms}$.

Fig. 1-5a. Transmission characteristic for different phase shifts. $L=30 \mathrm{~mm}, q=0$, $\phi=0, \pi / 4, \pi / 2,3 \pi / 4, \pi . L$ is total length of two gratings, $L=L_{1}+L_{2} \cdot q=\frac{L_{1}}{L_{1}+L_{2}}$ [8] 13

Fig. 1-5b. Transmission characteristic for different phase locations. $L=30 \mathrm{~mm}$, $q= \pm 0.30, \pm 0.32, \pm 0.35, \phi=\pi .[8]$ 14

Fig. 1-6. Schematic diagram of coupling from laser diode to fiber using hemispherical lens and LPG. [11] 16

Fig. 1-7a. Principle of operation of the wavelength-selective coupler using two longperiod gratings and a cladding-mode coupling region. [12]

Fig. 1-7b. Measured transmission outputs at port 1 and port 2 of the coupler. Side lobes can be eliminated by apodization technique. [12]

Fig. 1-8. (a) wavelength-dependent delay is produced by reflecting of the core mode at variable wavelength-dependent position in the case of Bragg grating. 
(b) wavelength-dependent delay is produced by coupling the core mode into a cladding mode, with larger group velocity, at variable wavelength-dependent position in the case

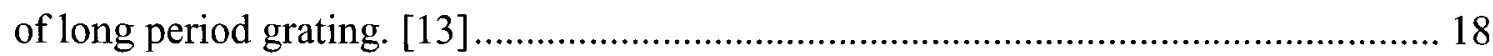

Fig. 1-9. Plot of LPG's as a function of wavelength for different cladding modes of the fiber. [14]

Fig. 1-10a. rejection band shift in wavelength for different values of LPG temperature $\left(22.7^{\circ} \mathrm{C}, 49.1^{\circ} \mathrm{C}, 74.0^{\circ} \mathrm{C}, 100.9^{\circ} \mathrm{C}, 127.3^{\circ} \mathrm{C}\right.$ and $149.7^{\circ} \mathrm{C}$ from left to right). a longperiod grating with $\Lambda=280 \mu \mathrm{m}$ was fabricated in Corning SMF-28. [15] ................... 22

Fig. 1-10b. shift of the rejection peaks with temperature. [15] 23

Fig. 1-11. Wavelength shift (a) and minimum transmission value of as a function of external refractive index. A period of grating is $400 \mu \mathrm{m}$, it is written in borongermanium co-doped fiber. [14]

Fig. 1-12. Transmission characteristics of LPG fiber. Solid curve (single peak resonance): no bend. Dashed curve (dual peak resonance): bent curvature $1.55 \mathrm{~m}^{-1}$. Grating period is

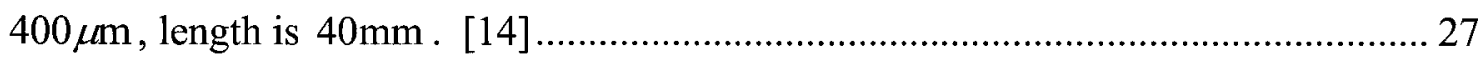

Fig. 1-13. Two LPGs in series acting as Mach-Zender interferometer. [14] .................. 27

Fig. 1-14. Transmission characteristics of two LPGs in series. (a) separation is $50 \mathrm{~mm}$, (b)

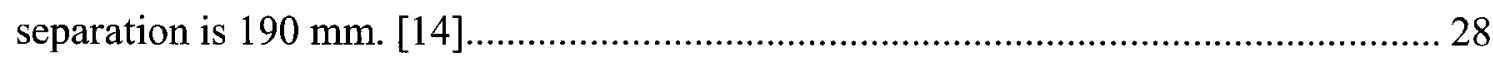

Fig. 2-1. FBG reflectivity with constant coupling coefficient. ....................................... 46

Fig. 2-2. $\quad \mathrm{M}$ is a transfer matrix for uniform grating................................................... 47

Fig. 2-3. FBG reflectivity with weak coupling coefficient. ............................................ 50

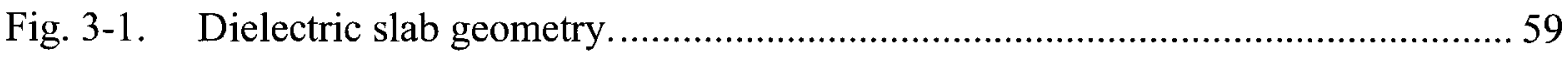

Fig. 3-2. Analogy between slab waveguide problem (Helmholtz equation) and square well potential problem (Schrödinger equation).

Fig. 3-3a. Successive perturbation approach to the 3-layer slab waveguide generates a

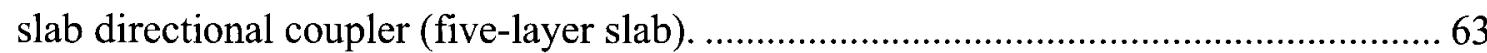

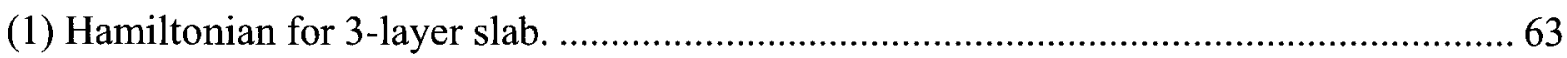

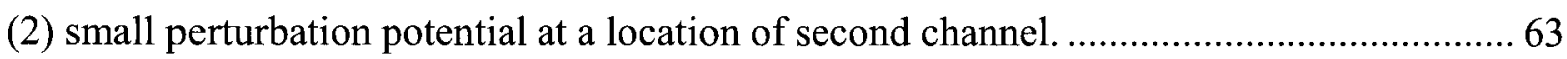


(3) total Hamiltonian combined corresponds to 5-layer slab profile with small refractive

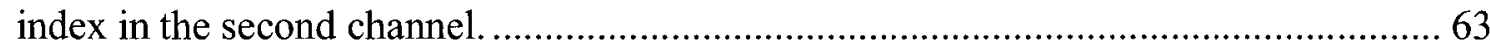

(4), (5) second step of increment results in larger refractive index in the second channel.... 63

Fig. 3-3b. By repeating perturbative increment many times, we can build up appropriate

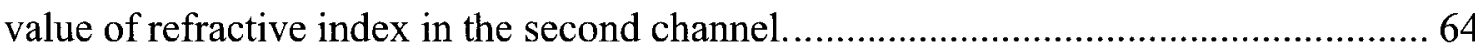

Fig. 3-4. Refractive index profile in 5-layer slab. Case 1, modes are guided in the second channel of the slab. Case 2, modes are evanescent in the second channel of the slab.... 66

Fig. 3-5. One channel of the slab. Generic characteristic equation satisfying phase consistency conditions

Fig. 3-6a. Electric field distribution between two channels for even mode.................... 77

Fig. 3-6b. Electric field distribution between two channels for odd mode. ..................... 78

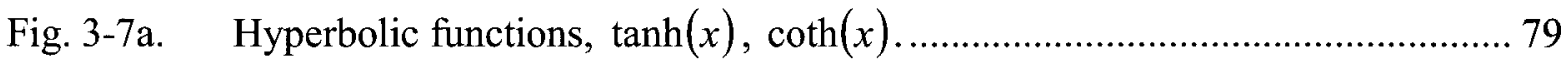

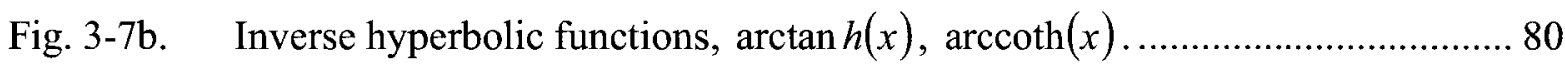

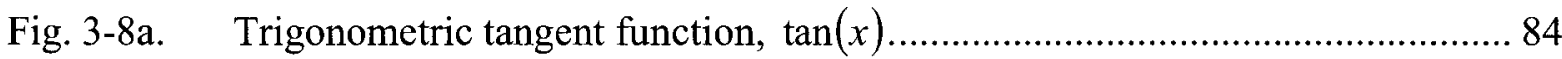

Fig. 3-8b. Proper inverse function of trigonometric tangent, $\tan ^{-1}(x)$ is multivalued

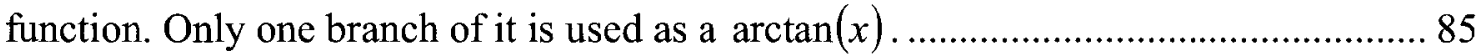

Fig. 3-9. Phase $\phi_{3}$ evolution diagram. Between $\phi_{3}=0$ and $\phi_{3}=\infty$ propagation constant, $\beta$ and modal index, $n_{\text {eff }}$ vary significantly, perturbation theory is not valid in this regime.

\section{7}

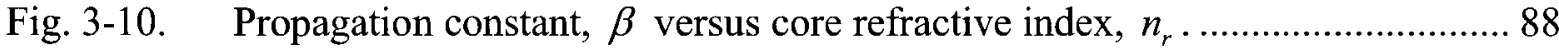

(a) perturbative calculation when $n_{r}$ increases. Before $n_{r 0}$ solution is correct. .................. 88

(b) perturbative calculation when $n_{r}$ decreases. After $n_{r 1}$ solution is correct. .................. 88

(c) case (a) and (b) combined give "hysteresis" loop, from which an approximate slope can

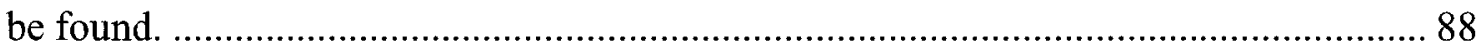

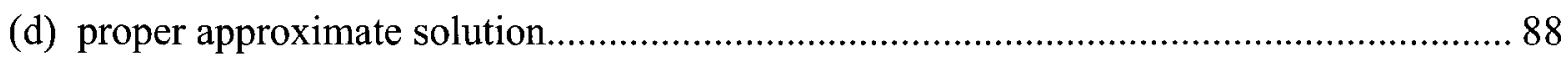

Fig. 4-1. Refractive index profile in a five-layer slab............................................. 93 
Fig. 4-2a. Approximate analytical solution. Modal indices versus core refractive index, $n_{r}$. Parameters: $\mathrm{v}=25 \mu \mathrm{m}, \mathrm{w}=5 \mu \mathrm{m}, \mathrm{s}=5 \mu \mathrm{m}, n_{c}=1.52, n_{b}=1.51, \lambda=1500 \mathrm{~nm} .97$

Fig. 4-2b. An illustration of slow and fast wave regions. When $n_{e f f}>n_{r}$ the mode velocity is smaller than phase velocity in the second channel, $\mathrm{v}_{p_{-} \text {mode }}<\mathrm{v}_{p}$. This region we call slow wave region. When $n_{e f f}<n_{r}$ the mode velocity is larger than phase velocity in the second channel, $\mathrm{v}_{p_{-} \text {mode }}>\mathrm{v}_{p}$. This region we call fast wave region.

Fig. 4-3. Phase $\phi_{B R}$ rotation at the right side boundary of the wide channel during the transition.

Fig. 4-4a, b. Modal indices versus core refractive index, $n_{r}$. Parameters: $\mathrm{v}=25 \mu \mathrm{m}$, $\mathrm{w}=5 \mu \mathrm{m}, \mathrm{s}=5 \mu \mathrm{m}, n_{c}=1.52, n_{b}=1.51, \lambda=1500 \mathrm{~nm}$

(a) Top plot is approximate analytical solution.

(b) Bottom plot is numerical simulation obtained with software package $\mathrm{C} 2 \mathrm{~V}$ 102

Fig. 4-5. Modal indices versus core refractive index $n_{r}$ for three different wavelengths.

Parameters: $\mathrm{v}=25 \mu m, \mathrm{w}=5 \mu m, \mathrm{~s}=5 \mu m, n_{c}=1.52, n_{b}=1.51$

Fig. 4-6. Surface plot $\left(n_{\text {eff } 0}-n_{\text {eff } 3}\right)$ versus core refractive index, $n_{r}$ and wavelength.

Parameters: $\mathrm{v}=25 \mu \mathrm{m}, \mathrm{w}=5 \mu \mathrm{m}, \mathrm{s}=5 \mu \mathrm{m}, n_{c}=1.52, n_{b}=1.51$

Fig. 4-7. Surface plot $\left(n_{\text {eff } 0}-n_{\text {eff } 5}\right)$ versus core refractive index, $n_{r}$ and wavelength.

Parameters: $\mathrm{v}=25 \mu \mathrm{m}, \mathrm{w}=5 \mu \mathrm{m}, \mathrm{s}=5 \mu \mathrm{m}, n_{c}=1.52, n_{b}=1.51$ 108

Fig. 4-8. Plot phase matching curves versus wavelength for three values of core refractive index. Parameters: $\mathrm{v}=25 \mu \mathrm{m}, \mathrm{w}=5 \mu \mathrm{m}, \mathrm{s}=5 \mu \mathrm{m}, n_{c}=1.52, n_{b}=1.51$.

Fig. 4-9. Plot power transmission characteristic for LPG element versus $\left(\frac{\delta}{\kappa}\right)$ 113 $y(x)=T=1-\frac{\sin ^{2}\left(0.5 \pi \sqrt{1+x^{2}}\right)}{1+x^{2}}, x=\frac{\delta}{\kappa}$. Where $\delta$ is detuning from phase matching condition, $\kappa$ is coupling coefficient. 
Fig. 4-10. Maximum values of $\delta$ for top curve should be about $+\kappa\left(\lambda_{0}\right) \cdot \sqrt{3}$ and for bottom curve about $-\kappa\left(\lambda_{0}\right) \cdot \sqrt{3}$ for transmission characteristic modulation from 0 to 1 114

Fig. 4-11. Transmission characteristic of a five-layer slab with LPG for various core refractive indeces.

Fig. 4-12. Transmission characteristics, $T(\lambda)$ of a five-layer slab with LPG. Core refractive index is modulated:

Fig. 4-13. Modal indices versus core refractive index, $n_{r}$ for various widths of the core.

Parameters: $\mathrm{v}=25 \mu \mathrm{m}, \mathrm{s}=5 \mu \mathrm{m}, n_{c}=1.52, n_{b}=1.51, \lambda=1550 \mathrm{~nm}$

Fig. 4-14. Plot $\Lambda=\frac{\lambda}{n_{\text {eff } 0}-n_{\text {eff } 3}}$ versus wavelength for various widths of the core.

Parameters: $\mathrm{v}=25 \mu \mathrm{m}, \mathrm{w}=4.5 \mu \mathrm{m}, \mathrm{s}=5 \mu \mathrm{m}, n_{c}=1.52, n_{b}=1.51$

Fig. 4-15. Plot $\Lambda=\frac{\lambda}{n_{\text {eff } 0}-n_{\text {eff } 2}}$ versus wavelength for three values of core refractive

indeces. Parameters: $\mathrm{v}=25 \mu \mathrm{m}, \mathrm{s}=5 \mu \mathrm{m}, n_{c}=1.52, n_{b}=1.51$

Fig. 4-16. Modal indices versus core refractive index $n_{r}$ for various cladding layer widths. Parameters: $\mathrm{w}=25 \mu \mathrm{m}, \mathrm{s}=5 \mu \mathrm{m}, n_{c}=1.52, n_{b}=1.51, \lambda=1550 \mathrm{~nm}$

Fig. 4-17. Plot $\Lambda=\frac{\lambda}{n_{\text {eff } 0}-n_{\text {eff }} m}$ versus wavelength for various cladding layer widths and mode coupling. Parameters:, $\mathrm{w}=5 \mu \mathrm{m}, \mathrm{s}=5 \mu \mathrm{m}, n_{c}=1.52, n_{b}=1.51$.

Fig. 4-18. Modal indices versus core refractive index $n_{r}$ for various separations.

Parameters: $\mathrm{v}=25 \mu \mathrm{m}, \mathrm{w}=5 \mu \mathrm{m}, n_{c}=1.52, n_{b}=1.51, \lambda=1550 \mathrm{~nm}$

Fig. 4-19. Plot $\Lambda=\frac{\lambda}{n_{\text {eff } 0}-n_{\text {eff } 3}}$ versus wavelength for various separations. Parameters:

$$
\mathrm{v}=25 \mu \mathrm{m}, \mathrm{W}=5 \mu \mathrm{m}, n_{\mathrm{c}}=1.52, n_{b}=1.51, \lambda=1550 \mathrm{~nm}
$$


Fig. 4-20. Plot $\Lambda=\frac{\lambda}{n_{\text {eff } 0}-n_{\text {eff } 3}}$ versus wavelength for two initial values of core refractive index. Parameters: $\mathrm{v}=25 \mu \mathrm{m}, \mathrm{w}=5 \mu \mathrm{m}, \mathrm{s}=3 \mu \mathrm{m}, n_{c}=1.52, n_{b}=1.51$. 132

Fig. 4-21. Modal indices versus core refractive index $n_{r}$ for various cladding refractive indeces $n_{c}$. Parameters: $\mathrm{v}=25 \mu \mathrm{m}, \mathrm{w}=5 \mu \mathrm{m}, \mathrm{s}=5 \mu \mathrm{m}, n_{b}=1.51, \lambda=1550 \mathrm{~nm} \ldots 134$

Fig. 4-22. Plot $\Lambda=\frac{\lambda}{n_{\text {eff } 0}-n_{\text {eff } 3}}$ versus wavelength for two initial values of core refractive index. Parameters: $\mathrm{v}=25 \mu \mathrm{m}, \mathrm{w}=5 \mu \mathrm{m}, \mathrm{s}=5 \mu \mathrm{m}, n_{c}=1.52, n_{b}=1.51$. 136

Fig. 4-23. Transmission characteristic wavelength for two initial values of core refractive index. Parameters: $\mathrm{v}=25 \mu \mathrm{m}, \mathrm{w}=5 \mu \mathrm{m}, \mathrm{s}=5 \mu \mathrm{m}, n_{c}=1.52, n_{b}=1.51$. 137

Fig. 4-24. Transmission characteristic for initial value of core refractive index 1.5191, $n_{r 0}=1.5191$. Parameters: $\mathrm{v}=25 \mu \mathrm{m}, \mathrm{w}=5 \mu \mathrm{m}, \mathrm{s}=3 \mu \mathrm{m}, n_{c}=1.52, n_{b}=1.51$, $\lambda=1550 \mathrm{~nm}$

Fig. 4-25. LPG slab made with spiral configuration. 140

Fig. 5-1a. Transversal and longitudinal section of the fiber with an additional layer deposited on the cladding. Refractive index of the deposited layer is higher than refractive index of the cladding. [42]

Fig. 5-1b. Effective index as a function of the overlay thickness of cladding modes. [42] 144

Fig. B-1. Diagram illustrates action of projection operator $P$ and operator $Q$ on a wavefunction $|\psi\rangle$.

Fig. D-1. Even modes.

top graphs, $y(Q)=\frac{2 Q^{2}-P^{2}}{P^{2}}$ and $y(Q)=\cos (Q)$

bottom graphs, $y(Q)=\frac{2 Q \sqrt{P^{2}-Q^{2}}}{P^{2}}$ and $y(Q)=\sin (Q)$

Proper solutions are marked by black dots. 
Fig. D-2. Odd modes.

top graphs, $y(Q)=\frac{P^{2}-2 Q^{2}}{P^{2}}$ and $y(Q)=\cos (Q)$

bottom graphs, $y(Q)=-\frac{2 Q \sqrt{P^{2}-Q^{2}}}{P^{2}}$ and $y(Q)=\sin (Q)$

Proper solutions are marked by black dots.

Fig. D-3. Cosine function, its second and forth order expansion.

Fig. D-4. Even and odd modes. Graphs $y(Q)=\frac{2 Q^{2}-P^{2}}{P^{2}}, y(Q)=\frac{P^{2}-2 Q^{2}}{P^{2}}$ and $y(Q)=\cos (Q)$. For even modes solutions are on negative slope of cosine, in intervals: $[0.5 \pi, \pi],[2.5 \pi, 3 \pi],[4 \pi, 4.5 \pi]$. For odd modes solutions are on positive slope of cosine, in intervals: $[1.5 \pi, 2 \pi],[3.5 \pi, 4 \pi],[5 \pi, 5.5 \pi]$

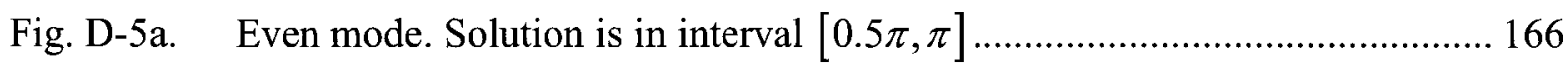

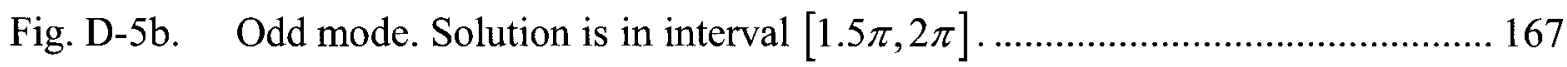

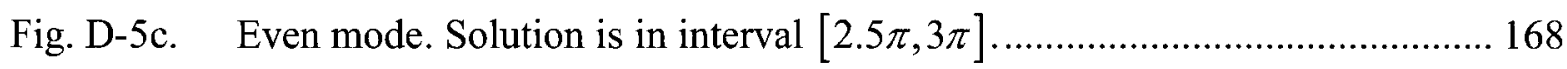

Fig. D-5d. Even mode. Solution is in interval $[4 \pi, 4.5 \pi]$. Odd mode. Solution is in interval $[3.5 \pi, 4 \pi]$

Fig. D-5e. Odd mode. Solution is in interval $[5 \pi, 5.5 \pi]$ 170 


\section{List of Symbols and Acronyms}

$\begin{array}{ll}\text { BG } & \text { Bragg grating } \\ \text { FBG } & \text { fiber Bragg grating } \\ \text { LPG } & \text { long period grating } \\ \text { RHS } & \text { right hand side } \\ \text { LHS } & \text { left hand side } \\ \text { EDFA } & \text { erbium doped fiber amplifier } \\ \text { DWDM } & \text { dense wavelength division multiplexing } \\ \text { CMT } & \text { coupled mode theory } \\ \text { PT } & \text { perturbation theory } \\ u . r . i . & \text { units of refractive index } \\ \beta & \\ \kappa & \text { propagation constant } \\ \chi & \text { coupling coefficient } \\ \lambda & \text { electric susceptibility } \\ \Lambda & \text { wavelength } \\ n & \text { grating period } \\ \vec{k} & \text { refractive index } \\ \hbar & \text { wave-vector } \\ \varepsilon_{0} & \text { Plank constant } \\ \mu_{0} & \text { permittivity of free space } \\ \vec{E} & \text { permeability of free space } \\ \vec{H} & \text { electric field amplitude } \\ \vec{D} & \text { magnetic field amplitude } \\ \vec{B} & \text { electric flux density } \\ \vec{P} & \text { magnetic flux density } \\ & \text { polarizability (of a medium) } \\ & \end{array}$

xiii 


\section{Thesis Outline}

It is a well known that the transmission characteristic of a long period fiber grating is very sensitive to environmental factors such as temperature, pressure, mechanical stress and external refractive index change [14].

Depositing an additional high refractive index layer to the cladding increases the sensitivity even further [27], [42]. Another factor that improves sensitivity is the operation near dual peak resonance, i.e. when the resonance conditions for a given grating period are satisfied for two distinct wavelengths. The wavelength separation between the two peaks exhibits a high sensitivity to bending which is 50 times higher than any previously reported sensors [55]. However, geometry and material constrains of the fiber result in functional limitations of the devices based on LPG filters. To overcome these limitations of the fiber, long-period waveguide grating (LPWG) have been proposed, [17]. Planar waveguides can be made with different shapes and sizes and different materials, which give additional control over transmission characteristics.

We want to combine all three factors above:

(a) instead of an additional layer to the cladding, we use two separate guiding channels. One channel acts as a core and another acts as an additional layer to the cladding, so the geometry is a five-layer slab structure (using the effective index approximation to transform the $3 \mathrm{D}$ real structure into its $2 \mathrm{D}$ equivalent);

(b) choose parameters in such a way as to operate near the dual peak regime. This should increase the sensitivity of the device;

(c) use a planar waveguide structure. This gives more geometrical and material parameters to control the transmission characteristics.

The aim of this work is to achieve high sensitivity to an internal index variation rather than an external one. If we succeed, the device will show high sensitivity to index changes of the order $\sim 10^{-6}$, which would be suitable for switching through nonlinear effects controlled by relatively low optical power. 
The thesis is organized as follows. Chapter 1 describes the basic properties and characteristics of the LPGs in fibers and in planar waveguides, and their various applications such as gain flattening and equalization, dispersion compensation and sensing. Then general nonlinear effects in optics and the existing possibilities to enhance them in fibers are reviewed. Based on these reviews we set our goal to design waveguide LPGs that are sensitive to small nonlinear effects and suitable for use in optical switching. Chapter 2 outlines general equations for coupled mode theory and its applications for contradirectional and codirectional couplers. The transfer matrix and transmission function for the case of codirectional coupling will be used later for the analysis of LPGs in a planar waveguide.

In chapter 3 we derive the characteristic equation for a nonsymmetrical five-layer slab for TE mode. For the sake of completeness we have started the derivation from Maxwell's equations. To find the exact propagation constant from the characteristic equations involves finding the roots of a complicated transcendental equation. Therefore an approximate estimation is desirable.

In chapter 4 , the derived characteristic equation is analyzed by applying a perturbative approach, i.e. starting from a symmetrical three-layer slab as an initial waveguide whose propagation constant is assumed to be known, we apply a small perturbation to the existing index profile to form the second channel and therefore form a five-layer slab. This chapter presents the comparison between the approximate solution and a numerical simulation. The remaining analysis of the waveguide is done numerically. We have introduced LPGs in the five-layer slab and examined its transmission characteristics. Then, the influence of geometrical parameters on transmission is studied. An optimal set of such parameters exists which makes the waveguide very sensitive to a small refractive index modulation. A summary of the results is presented in chapter 5 . The total length of the waveguide is found to be very large for any practical use. We discuss possibilities to reduce that length while keeping the transmission characteristic still sensitive to small refractive index changes. 


\section{Chapter 1 \\ Introduction}

Guided wave photonic components perform essential functions in modern optic communication systems and optical instrumentation. Among these devices a waveguide grating, i.e. an optical waveguiding structures with (quasi) periodic refractive index perturbation, offers $\lambda$-dependent transmission function over a wide range of parameters. It this chapter we go over the basic principle of operation for the long period grating and review its applications. We focus our attention on sensor properties of LPGs and on the possibility to enhance nonlinear effects in the fiber.

\subsection{Long Period Gratings in Fibers}

A long period grating (LPG) is a periodic variation, usually sinusoidal, of the waveguide refractive index with a period much larger than wavelength of the signal. For a twodimensional structure where propagation occurs along the z-axis and the waveguide crosssection along the $\mathrm{x}$-axis is otherwise invariant:

$n(x, z)=n_{0}(x)+\Delta n \cdot \sin \left(\frac{2 \pi}{\Lambda} z\right)$

$n_{0}(x)$ is the unperturbed refractive index, i.e. the index profile before the grating is introduced,

$\Delta n$ is the index modulation amplitude, $\Lambda$ is the grating period.

The typical range for the LPG period at optical wavelengths is $100 \mu m-1000 \mu m$. The main purpose of the LPG is to couple two modes propagating in the same direction. The condition for coupling to occur is called a phase matching condition, described by (1-2):

$\beta_{n}-\beta_{m}=\frac{2 \pi}{\Lambda}$

where 
$\beta_{n}, \beta_{m}$ are the propagation constants of modes $n, m$ respectively such that the mode field of mode $m$ at frequency $\omega$ is expressed by

$\varphi_{m}(x, z)=F(x) \exp \left[-j\left(\omega t-\beta_{m} z\right)\right]$

In the particle picture of light (1-1) can be viewed as a momentum conservation condition. Multiplying (1-1) by $\hbar$ (Plank constant) and keeping in mind that the propagation constant is the z-component of the wavevector $(\vec{k})$ of each mode, we have (see Fig. 1-1):

$$
\begin{aligned}
& \hbar k_{z n}=\hbar k_{z m}+\hbar \frac{2 \pi}{\Lambda} \\
& p_{z n}=p_{z m}+\hbar \frac{2 \pi}{\Lambda}
\end{aligned}
$$

Equation (1-3) tells that a photon belonging to mode $\mathrm{n}$ and having momentum $p_{z n}$ collides with and scatters off the grating. As a result of scattering, momentum $p_{z n}$ has been transferred partially to a new photon belonging to mode $m$ having momentum $p_{z m}$ and partially to the grating $\hbar \frac{2 \pi}{\Lambda}$. The role of the grating is to help in the transition $p_{z n} \rightarrow p_{z m}$. When $\Lambda$ satisfies (1-3) then the probability of such transition is the maximum possible. Strictly speaking (1-3) is the momentum conservation for the z-component only. However for propagating modes the transverse component is very small, $k_{x}<<k_{z}, k_{z} \approx|\vec{k}|$. Another assumption is that our grating is purely longitudinal (see (1-1)), i.e. it does not modify the index profile along the $\mathrm{x}$-axis. If the grating is slightly tilted, i.e. it modifies the index along the $\mathrm{x}$-axis, then momentum conservation is described by two equations,

$$
\begin{aligned}
& p_{z n}=p_{z m}+\hbar \frac{2 \pi}{\Lambda_{z}} \\
& p_{x n}=p_{x m}+\hbar \frac{2 \pi}{\Lambda_{x}}
\end{aligned}
$$




\section{before scattering off LPG}

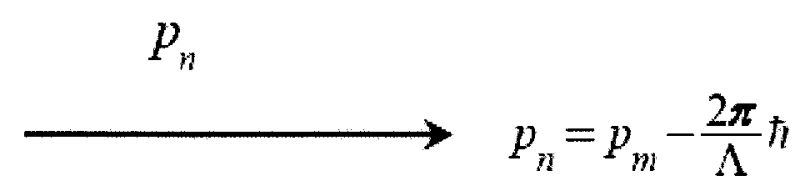

after scattering off LPG
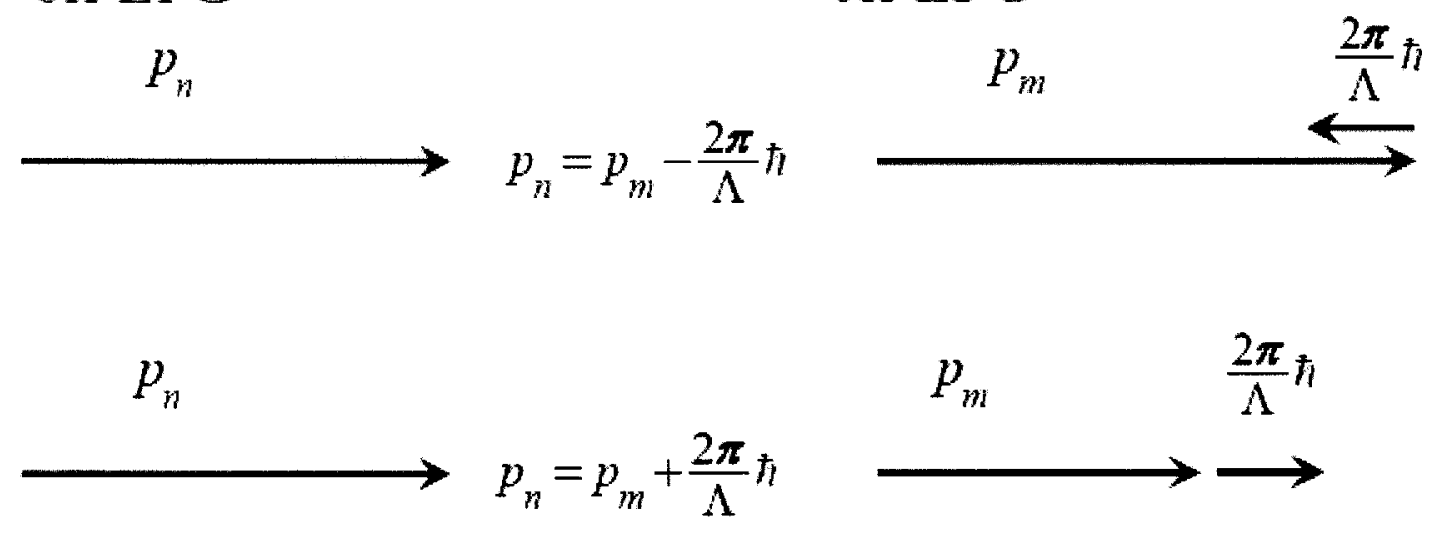

$$
\left|\vec{p}_{n}\right|=\frac{2 \pi}{\lambda_{n}} \hbar
$$

$$
\left|\vec{p}_{m}\right|=\frac{2 \pi}{\lambda_{m}} \hbar
$$
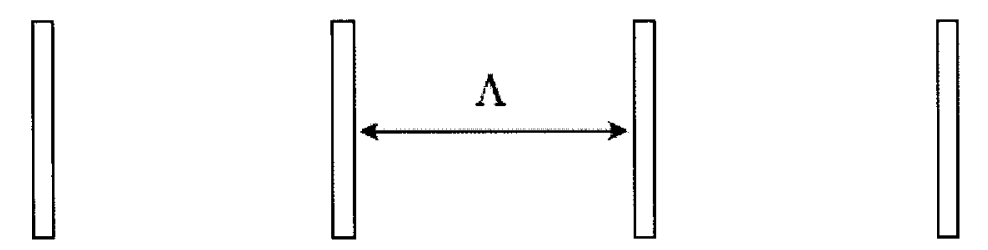

Fig. 1-1. Scattering off LPG in fiber. Momentum conservation in photon picture.

In the case when a LPG couples mode $n$, whose field is mainly distributed in the core of a waveguide, with mode $\mathrm{m}$ whose field is mainly distributed in the cladding, the transmission characteristic has rejection bands for a set of wavelengths satisfying a phase-matching condition for all possible cladding modes. This is because propagation in the cladding is characterized by higher losses, so modes coupled to the cladding eventually die out, producing a rejection band, see Fig. 1-2a, b. 


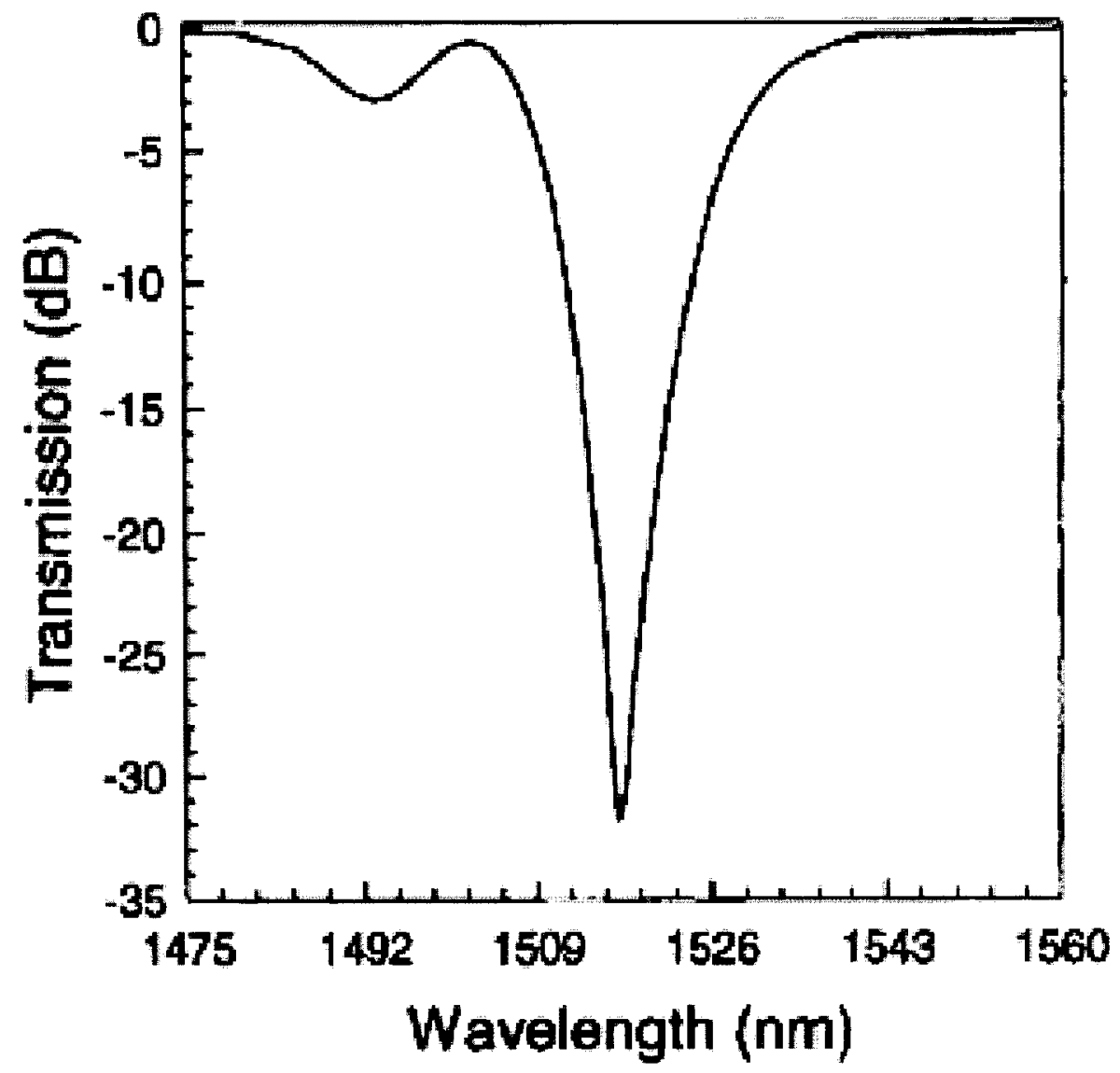

Fig. 1-2a. Transmission characteristic UV-induced LPG in fiber. [1] 


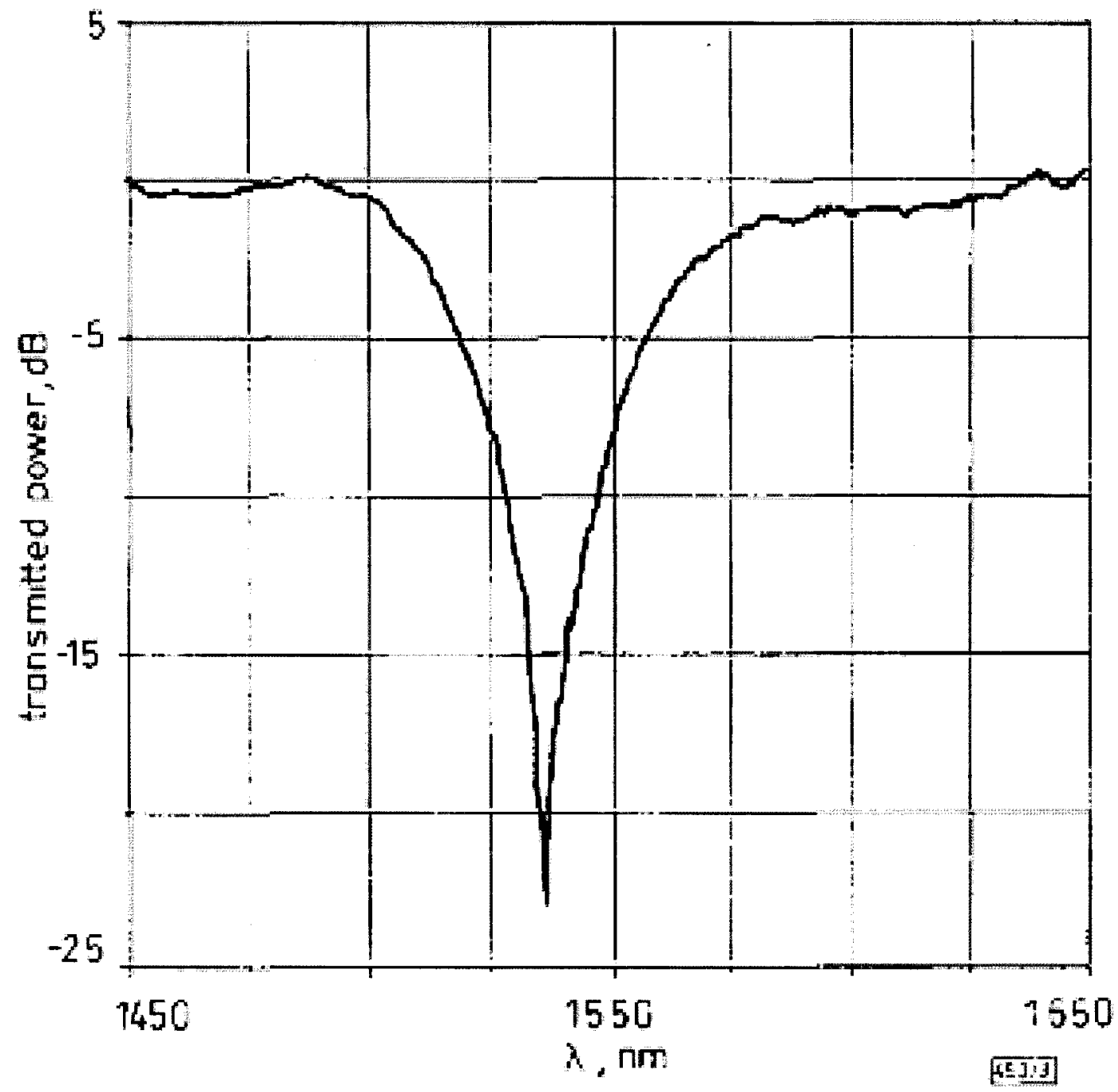

Fig. 1-2b. Transmission characteristic LPG fabricated by periodic deformation of the core of the fiber using $\mathrm{CO}_{2}$ laser. [2]

In (1-4a) the plus sign is used, however for a vector $\vec{K}_{G},\left|\vec{K}_{G}\right|=\frac{2 \pi}{\Lambda}$ there is no defined direction, so we can have both signs in the expression:

$$
p_{z n}=p_{z m} \pm \hbar \frac{2 \pi}{\Lambda_{z}}
$$


but one of the solutions, usually with + is not physically possible because then $\beta>|\vec{k}|$ in the core material.

There will be several analogies in the thesis between the wave representation of optical fields and the particle-like behavior of photons. See [56] for more details on the wave-particle duality of light fields.

\subsection{Applications of Long Period Gratings}

LPGs have found numerous applications in telecommunications including gain flattening, dispersion compensation as well as in precision measurements and monitoring as a sensor of temperature, pressure, strain, and external refractive index. Some of these applications are listed below.

\subsubsection{Rejection filter, bandpass filter, tunable filter. [1] - [4]}

LPGs were first described by Vengsarkar and his coworkers [1] as band rejection filters mentioned above. Fabricated photoinduced LPGs in fibers (with $\Delta n \sim 10^{-3}$ ) act as spectrally selective loss filters having insertion losses $<0.2 \mathrm{~dB}$, backreflections $<-80 \mathrm{~dB}$, polarization dependent losses $<0.02 \mathrm{~dB}$. The rejection bandwidths are typically large $(10-50 \mathrm{~nm})$. Another method of fabrication of LPGs is by the periodic deformation of the fiber using $\mathrm{CO}_{2}$ laser irradiation or arc fusion or mechanically induced stress was demonstrated [2]. Index modulations in this case can be pretty large $(\Delta n=0.1)$ and strong LPGs can be made with shorter lengths (few mm). For instance, a 6-mm long LPG ( $\Lambda=610 \mu m, \quad N=10)$ fabricated with $\mathrm{CO}_{2}$ laser-induced deformation has a $-23 \mathrm{~dB}$ loss peak, a 3-dB bandwidth equal to $48 \mathrm{~nm}$, and an insertion loss $<0.3 \mathrm{~dB}$. For comparison, the transmission characteristics of rejection filters fabricated with both methods are shown in Fig. 1-2a, b. A bandpass filter can be constructed from two long period gratings with a mode blocker in the core between the LPGs [3], see Fig. 1-3a. The first LPG couples light from the core to the cladding and a second LPG couples it back to the core. 
To avoid Mach-Zender interference, this device must have only one transmission path. The second possible path is blocked by the core blocker. However for wavelengths satisfying the phase-matching condition, the cladding acts as a bypass around the core mode blocker. Light transmission through the filter can also be controlled by a refractive index modulator, attached to the cladding. The transmission characteristic of this bandpass filter is shown in Fig. 1-2b.

Such filters can be made electrically tunable by using for example a metal coating, [4]. When a DC current is applied (resistive power $\sim 0.6 \mathrm{~W}$ ) to the copper coating, the attenuation characteristic of the LPG-based filters decreases and shifts by $\sim 4 \mathrm{~nm}$. This effect is due to the temperature dependence of the refractive indices of both the core and cladding.

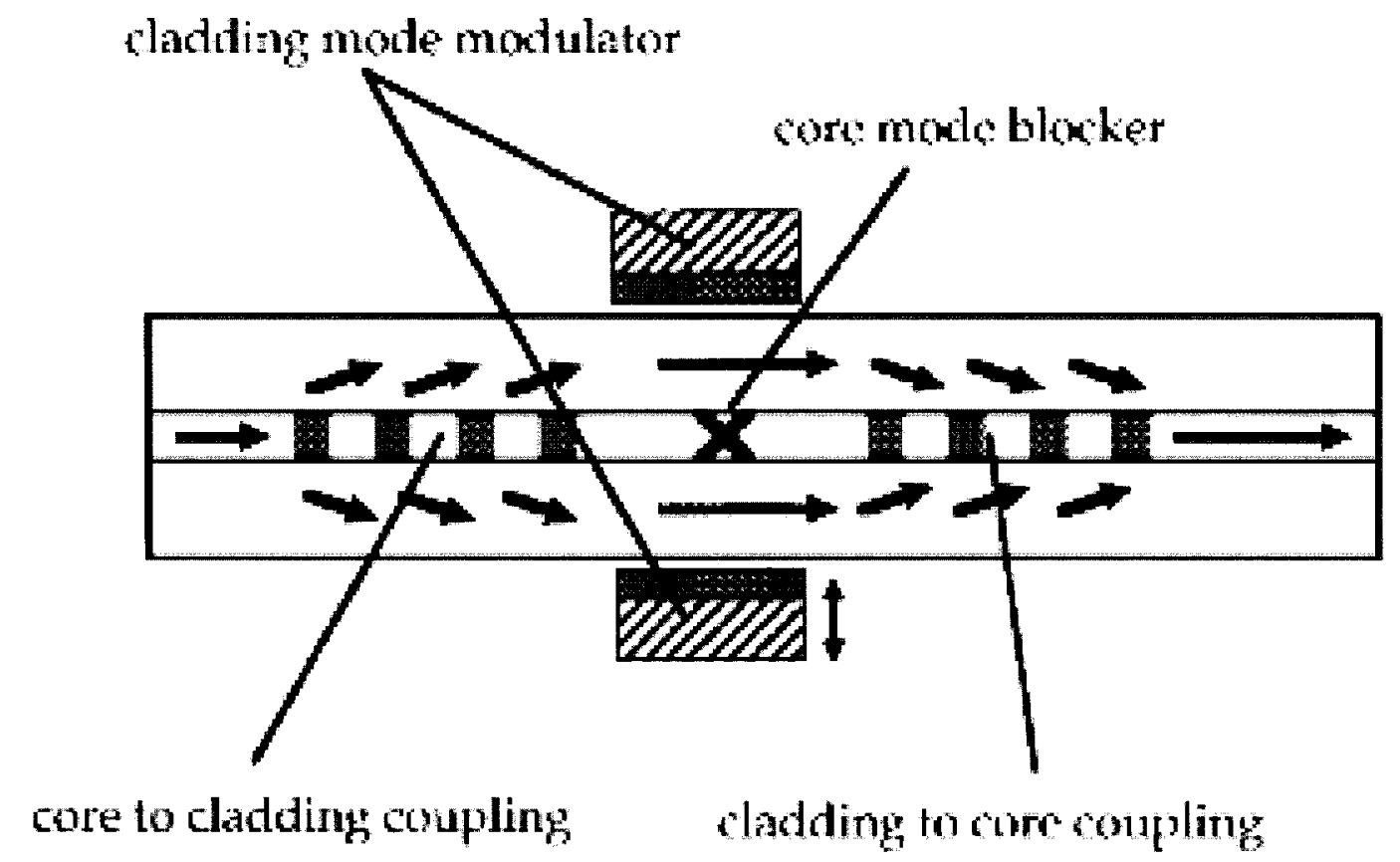

Fig. 1-3a. Band-pass filter scheme using two long period gratings in series. The core mode blocker prevents light from passing through the core. The cladding mode modulator controls the transmission through the device. [3] 


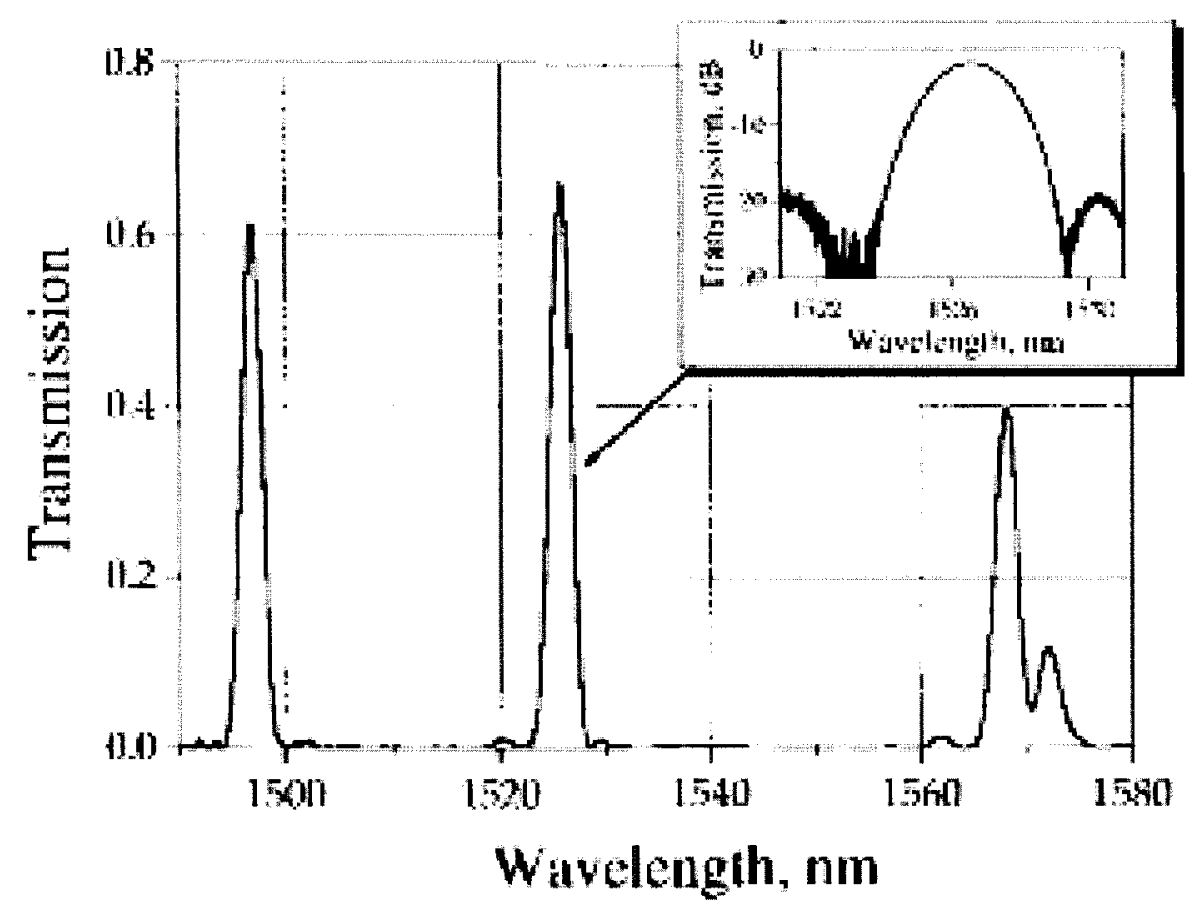

Fig. 1-3b. Measured transmission spectrum of bandpass filter showing the main passband channel at $\sim 1527 \mathrm{~nm}$ and adjacent channels from different cladding modes. The insert shows that the detailed spectrum of the central peak, which has a 2-nm bandwidth at $-3 \mathrm{~dB}$. [3]

\subsubsection{Gain flattening and equalizer. [5] - [10]}

With the fast development of dense wavelength division multiplexed (DWDM) optical communication systems many research topics have focused on the erbium doped fiber amplifier (EDFA), which is one of the main devices allowing the deployment of WDM. By pumping erbium doped fiber at $980 \mathrm{~nm}$ or $1480 \mathrm{~nm}$ with a semiconductor laser, it provides a strong wide emission band $(40 \mathrm{~nm})$ centered at $1550 \mathrm{~nm}$ [5]. The diagram of $\mathrm{Er}^{3+}$-ions energy levels, which are active in the amplifier, are shown in Fig. 1-4. The EDFA has low noise, high optical power, wide bandwidth, polarization independence and allows multi-stage amplification schemes. However, its gain spectrum is not equal over the bandwidth of the emission spectrum. 


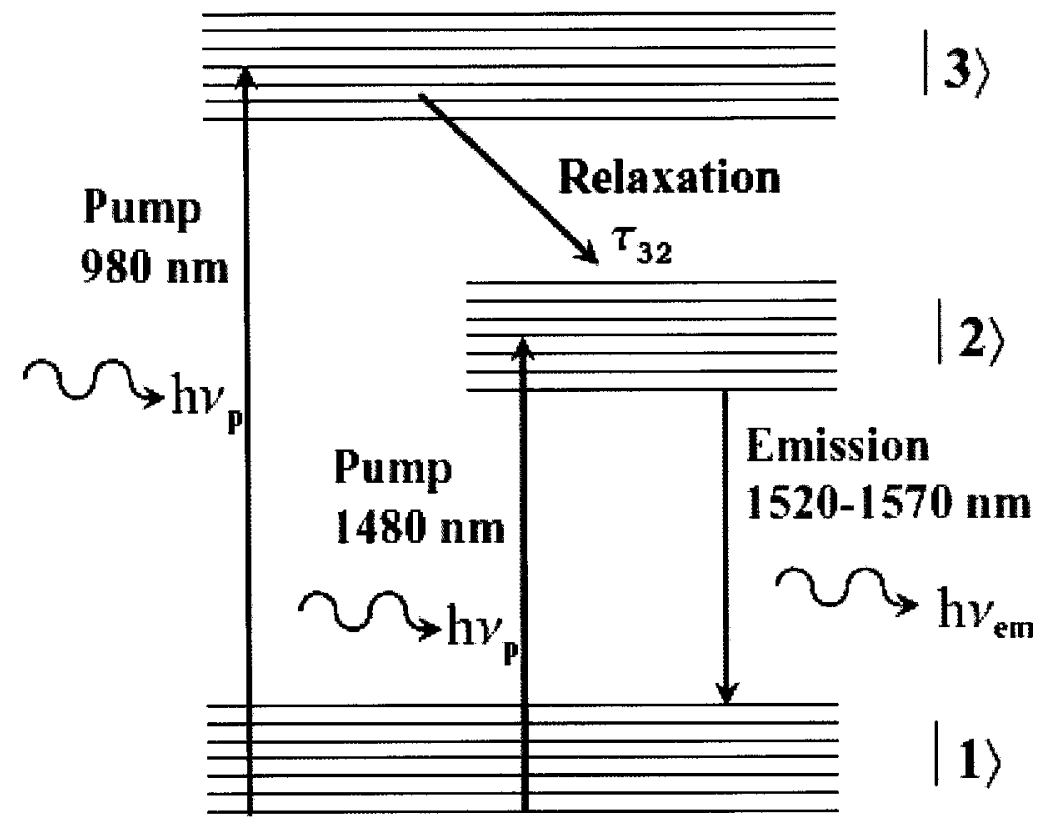

Fig. 1-4. energy levels of $\mathrm{Er}^{3+}$ ions used in EDFA. Suitable pumps are at $\sim 980 \mathrm{~nm}$ and $\sim 1480 \mathrm{~nm} . \tau_{21} \gg \tau_{32}, \tau_{21} \sim 10 \mathrm{~ms}$.

This leads to signal distortion and reduces signal to noise performance. Several methods have been proposed to equalize the EDFA gain spectrum. One is by additional doping by other ions, for example a fluoride based EDFA has better flatness of the gain curve [6]. However, the bandwidth of such co-dopant is limited to only 25-28 nm range. Another way is to use an external filter, which has a loss profile inverse to the gain of the EDFA, connected in series. It is advantageous to use a LPG for gain flattering since such a grating has small insertion loss and negligible back reflection [7]. To compensate for the peak of the EDFA gain at certain wavelengths, the LPG has to have a loss peak at the same wavelength, i.e. the period of the grating has to be chosen in such a way that the phase matching condition at that wavelength is satisfied. Then, the optical power from the core mode is coupled to the lossy cladding mode and the amount of loss is controlled by the index modulation and the total 
length of the grating. To compensate for several gain peaks, more than one LPG stage are usually required.

A single long period grating in a fiber has a peak loss unsuitable for the purpose of gain flattening, so it is highly desirable to control the width and shape of the loss peak to match the gain curve. It turns out that phase-shifted and cascaded long period gratings in a fiber allow to precisely design the spectral shape of LPG transmission characteristic [8] by choosing appropriate phase shift and separation between gratings. For example, the transmission characteristics of two typical LPGs when their separation is zero and when a phase shift is introduced in the middle of the combined LPG with values from 0 to $\pi$, is shown in Fig. 1-5a. The main loss peak is gradually displaced towards higher wavelengths with decreasing its magnitude, and simultaneously the side lobe peak is increased. At $\phi=\pi$ the main peak is split into two equal peaks. This is because destructive interference is converted into constructive interference at the phase matching wavelength due to a $\pi$-shift. Fig. $1-5 \mathrm{~b}$ shows the transmission characteristics when a $\pi$-shift is introduced at three different positions in the combined LPG. We see, that the phase shift location can turn a single sharp peak into a wide and flat response. Flattening of the gain of an EDFA with 3-dB bandwidth equal to $46 \mathrm{~nm}$ and (characteristic of the flatness) peak-to-peak ripple $0.9 \mathrm{~dB}$ has been experimentally achieved [9].

With phase shifted LPGs it is also possible to make the loss peak even sharper, which is very useful for sensor-like applications. 


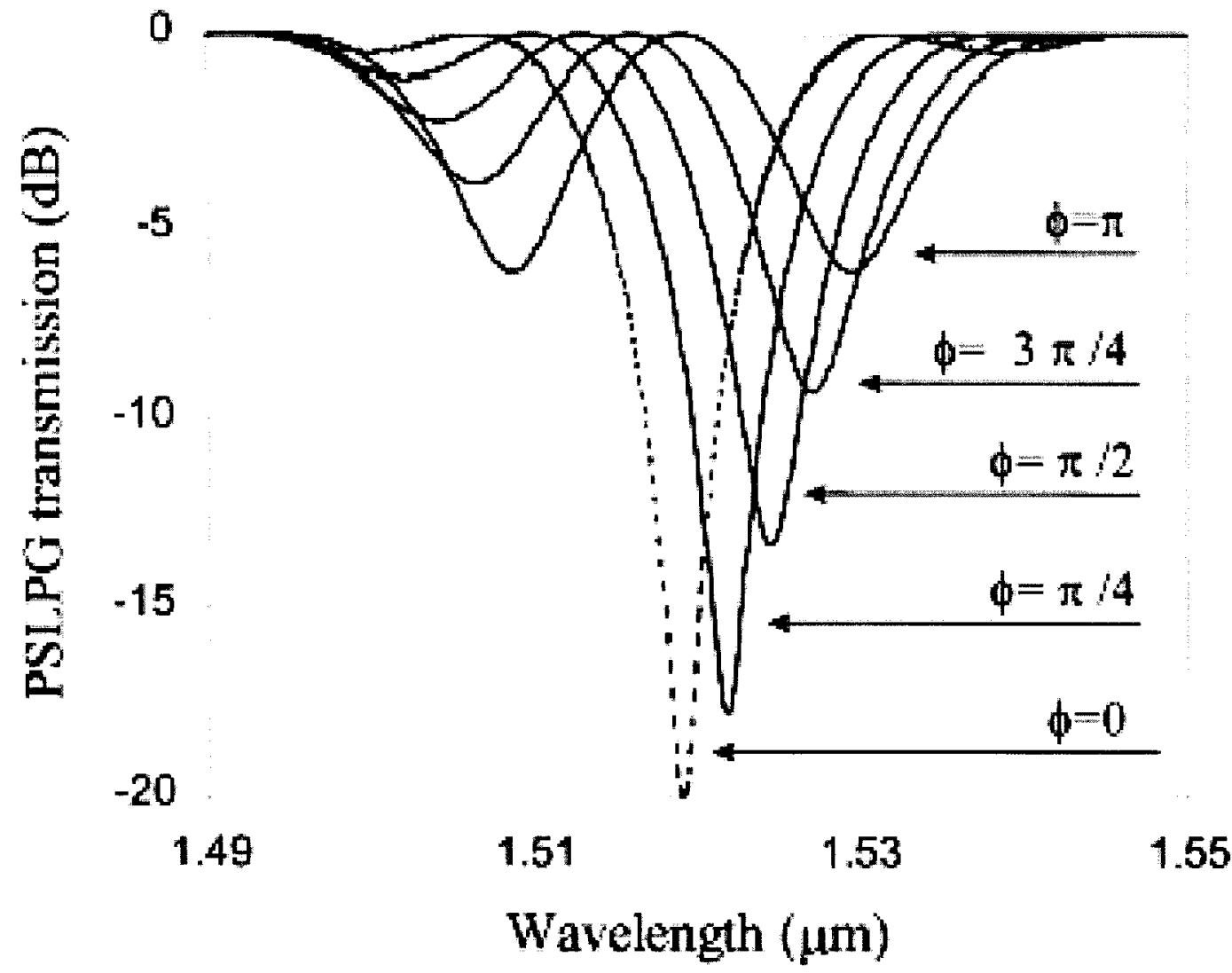

Fig. 1-5a. Transmission characteristic for different phase shifts. $L=30 \mathrm{~mm}, q=0$, $\phi=0, \pi / 4, \pi / 2,3 \pi / 4, \pi$. $L$ is total length of two gratings, $L=L_{1}+L_{2}$. $q=\frac{L_{1}}{L_{1}+L_{2}}[8]$ 


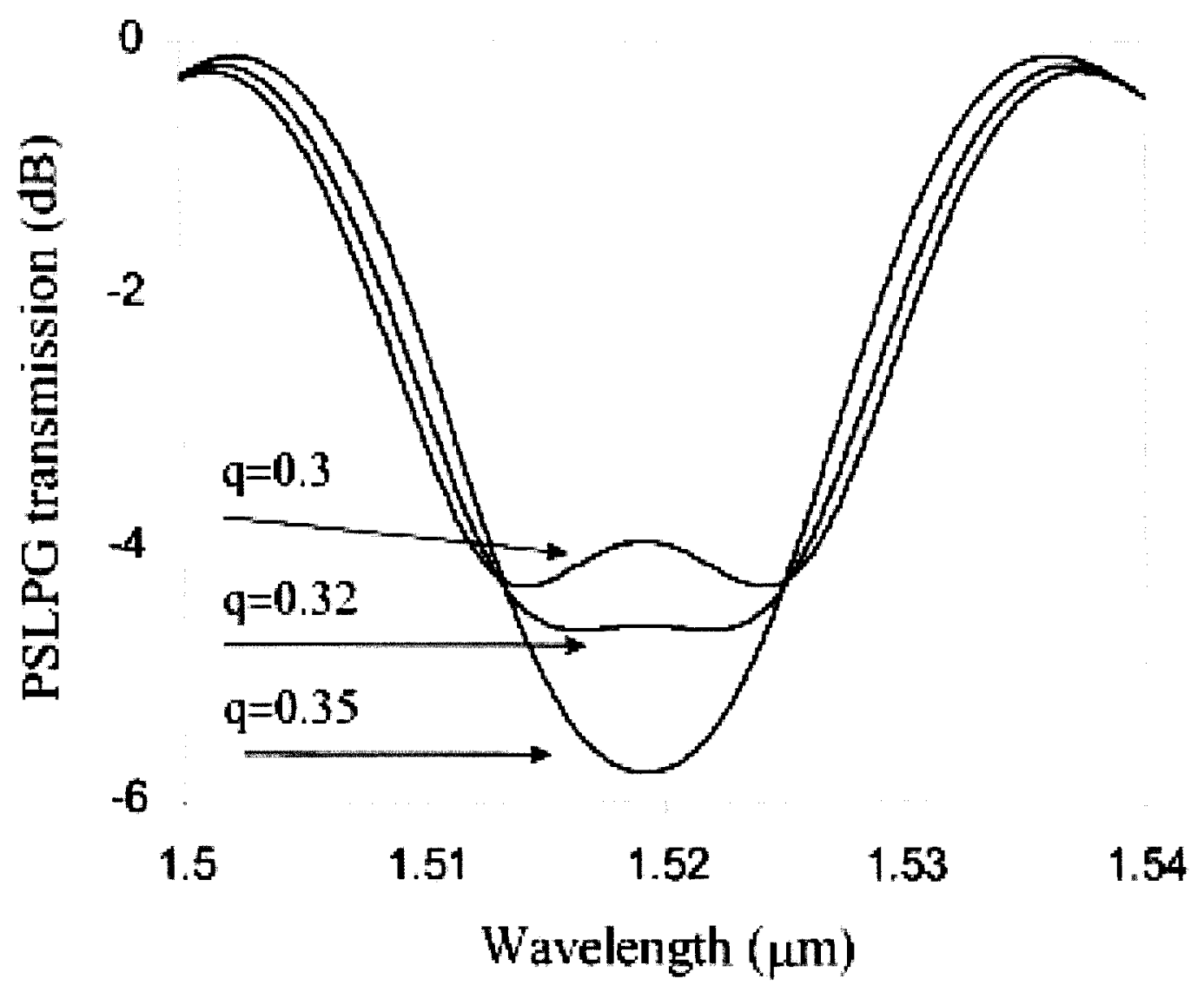

Fig. 1-5b. Transmission characteristic for different phase locations. $L=30 \mathrm{~mm}$, $q= \pm 0.30, \pm 0.32, \pm 0.35, \phi=\pi$. [8]

Recently, a novel technique was proposed to write the LPG on the EDFA itself [10]. The advantage of such novelty is that it provides not only gain flattening but also gain enhancement in $1530-1560 \mathrm{~nm}$ range. A possible explanation for this is the population inversion in the EDFA is redistributed among wavelengths. When many wavelengths propagate in EDFA, excited (due to pumping) $\mathrm{Er}^{3+}$ ions experience a transition back to the ground state while emitting light (stimulated emission). Different wavelengths actually 'compete' for the excited $\mathrm{Er}^{3+}$ ions. If for example the probability that wavelength $\lambda_{1}$ causes 
stimulated emission is higher than for wavelength $\lambda_{2}$, then the EDFA emits more light of $\lambda_{1}$ and less of $\lambda_{2}$ (this is the origin of non-flat gain profile). Now, if a long period grating is written in the body of amplifier. Light at wavelength $\lambda_{1}$ is partially coupled to the cladding, while light at $\lambda_{2}$ is not. Therefore, wavelength $\lambda_{1}$ participates less in the stimulated emission and more excited $\mathrm{Er}^{3+}$ ions are available for wavelength $\lambda_{2}$. Higher gain at $\lambda_{1}$ is slightly reduced, weaker gain at $\lambda_{2}$ is slightly enhanced. Such redistribution increases the average gain of the EDFA.

\subsubsection{Insertion light coupler. [11], [12]}

Optical coupling from a laser diode to a fiber, utilizing an integrated hemispherical lens and a long period grating has been proposed and implemented [11]. Rays emitted from an laser diode (see Fig. 1-6) at an output angle $\phi_{0}$ are coupled into the cladding of a receiving single mode fiber by a hemispherical fiber lens. Then, with the help of the LPG they are coupled into the fiber core. The advantage of this coupling scheme over others is the relatively large working distance between the lens and the laser diode, $\sim 100 \mu \mathrm{m}$, and the consequent reduction of optical backscattering up to $-60 \mathrm{~dB}$. For a conventional scheme without LPG, the working distance is $\sim 10 \mu \mathrm{m}$ and the backscattering is $-30 \mathrm{~dB}$.

A wavelength selective coupler which can be used as an add-drop multiplexer in DWDM system can also be implemented with LPGs [12], see Fig. 1-7a. The coupler consists of two closely placed fibers with LPGs on them. The input light is launched in the core of the first fiber. The first grating, LPG 1 couples the light into a cladding mode at a desired wavelength, while all other wavelengths propagate in the core.

The cladding mode propagates in the first cladding, couples to the cladding of the second fiber in coupling region (via evanescent coupling) and then through the LPG 2 into the core of the second fiber. Only selected wavelengths of the total input are coupled to port 2, and all other wavelengths are coupled to port 1 . Such add-drop multiplexer is almost free from backreflections, the three stage coupling ensures very high isolation between the channels 


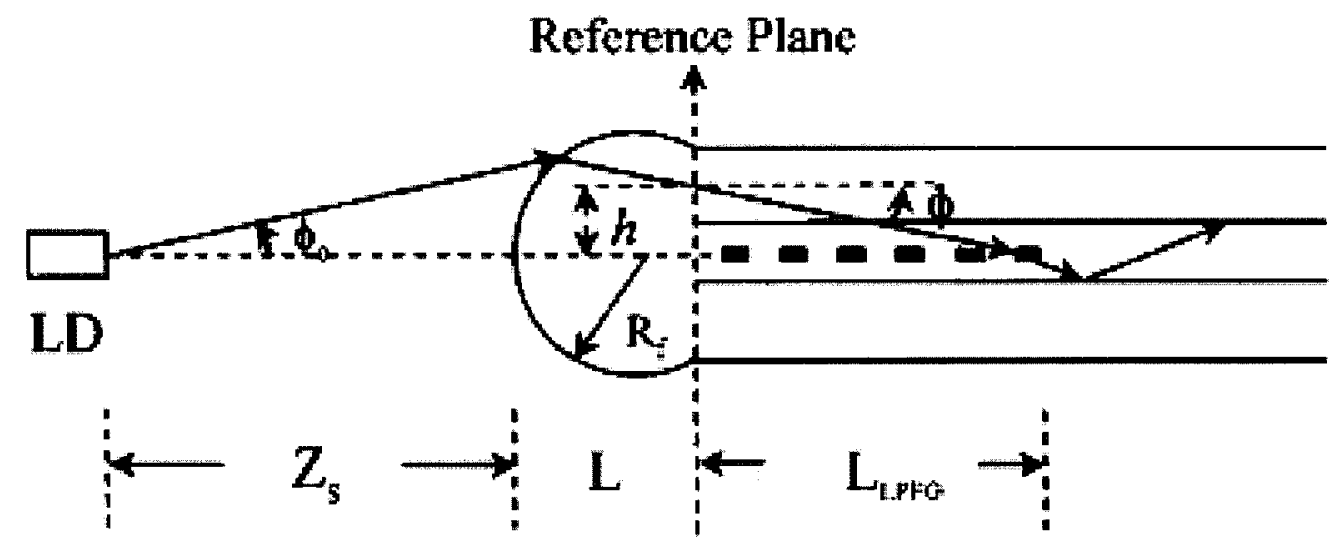

Fig. 1-6. Schematic diagram of coupling from laser diode to fiber using hemispherical lens and LPG. [11]

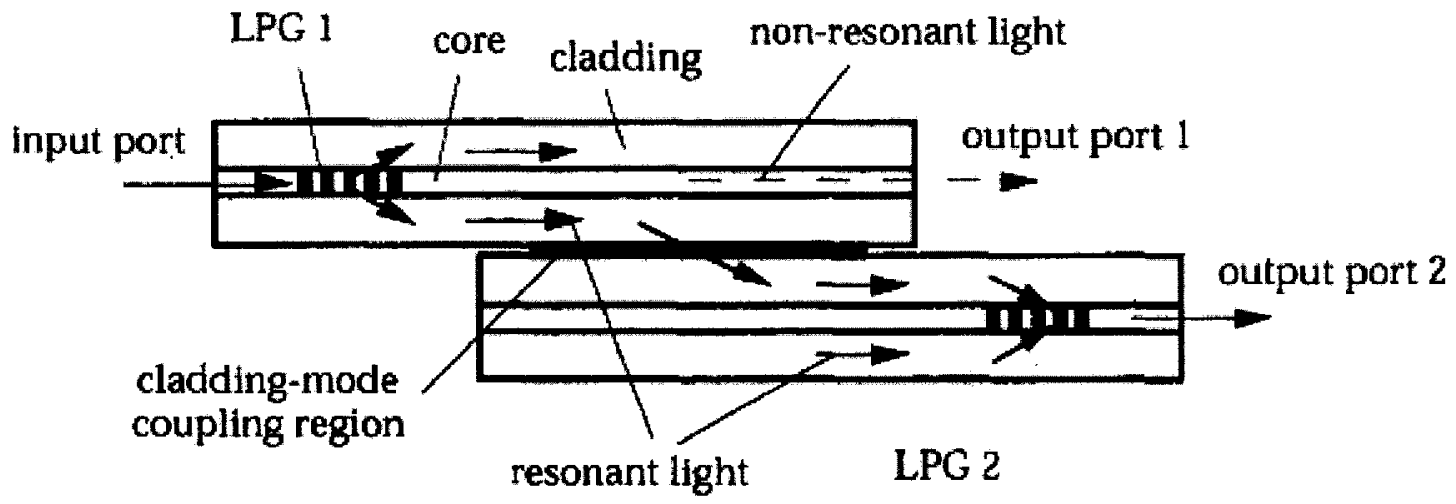

Fig. 1-7a. Principle of operation of the wavelength-selective coupler using two longperiod gratings and a cladding-mode coupling region. [12] 


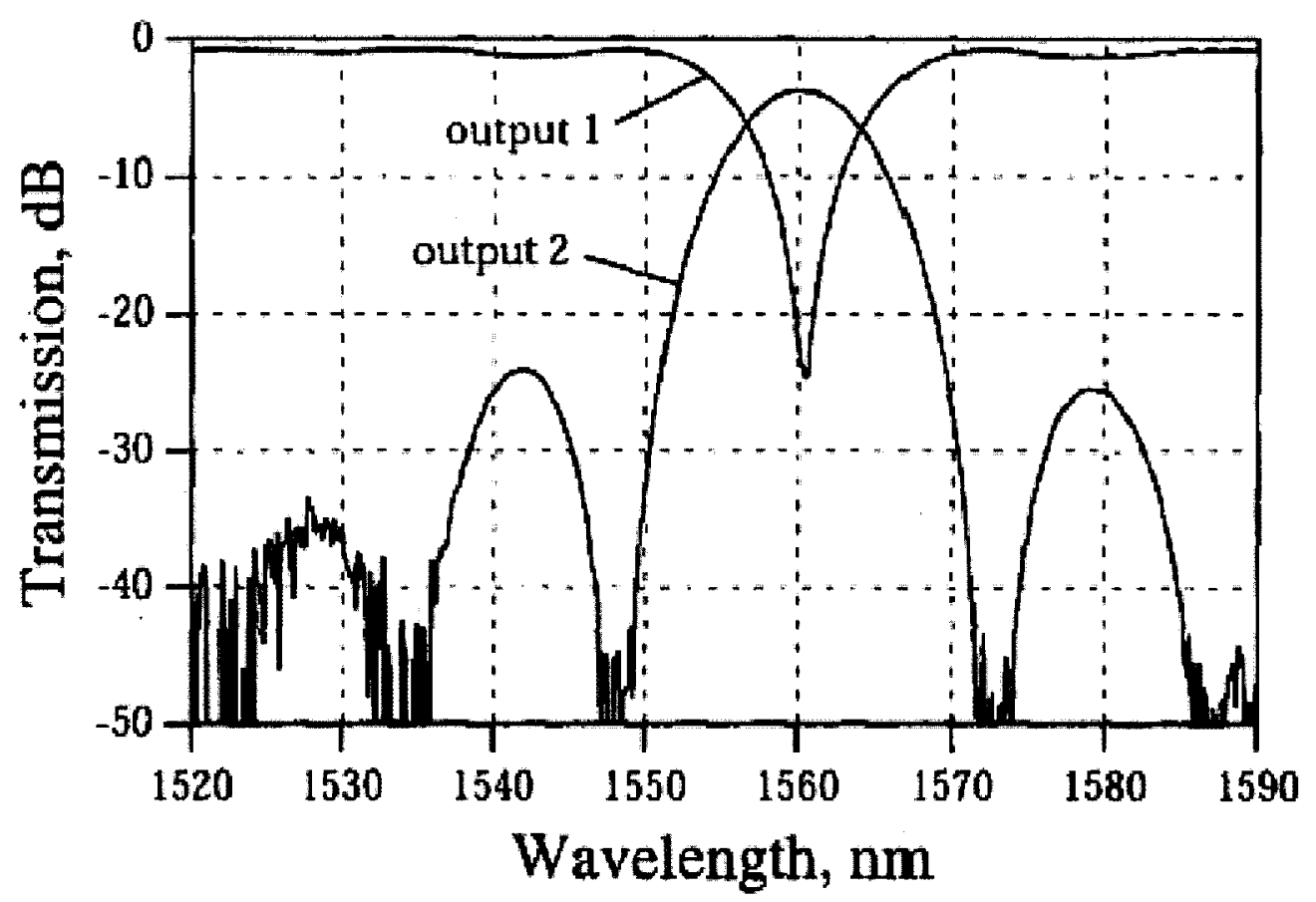

Fig. 1-7b. Measured transmission outputs at port 1 and port 2 of the coupler. Side lobes can be eliminated by apodization technique. [12]

$(>40 \mathrm{~dB})$, and overall insertion losses are very small. Those advantages make it attractive to use in DWDM system. Its spectrum is shown in Fig. 1-7b.

\subsubsection{Dispersion compensation. [13]}

Since an optical fiber is a dispersive media, different wavelength components of the signal have non-equal group velocity. After propagation over a long distance two distinct pulses can be eventually overlapped leading to lost signal. To prevent this, dispersion compensation is needed. 
Chirped fiber Bragg gratings (FBG) can compensate for dispersion as shown in Fig. 1-8a. Bragg reflection of different wavelength components of the signal takes place at different locations in the Bragg grating.

(a)
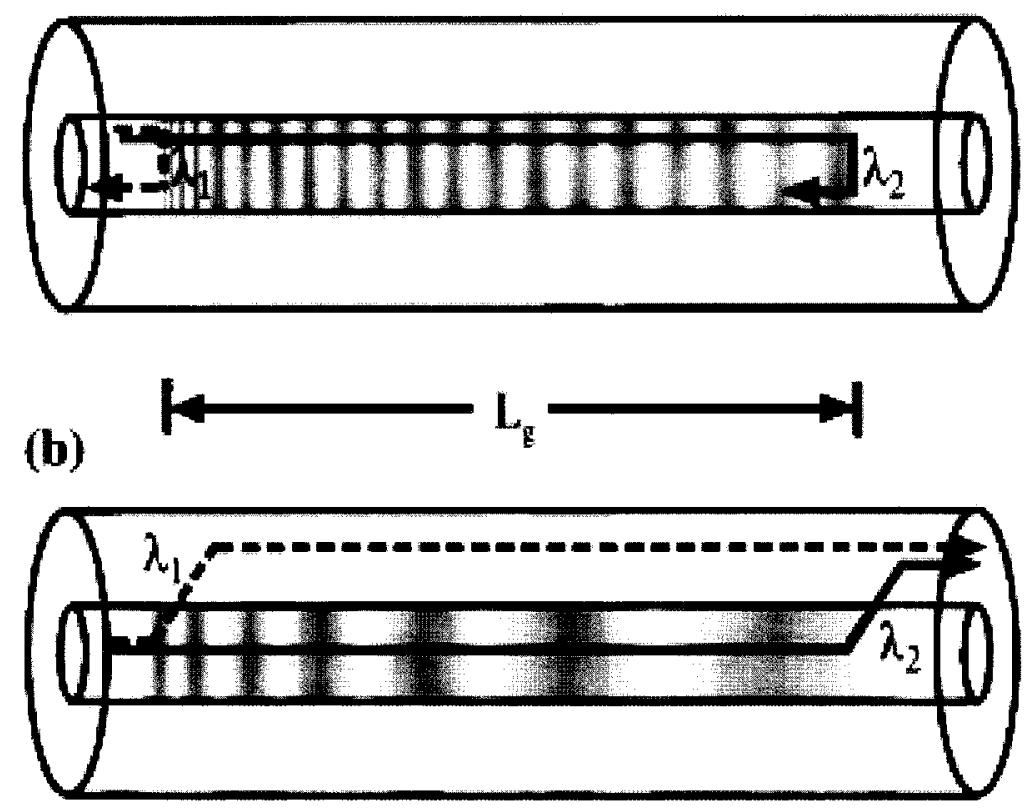

Fig. 1-8. (a) wavelength-dependent delay is produced by reflecting of the core mode at variable wavelength-dependent position in the case of Bragg grating.

(b) wavelength-dependent delay is produced by coupling the core mode into a cladding mode, with larger group velocity, at variable wavelength-dependent position in the case of long period grating. [13]

Some wavelengths propagate over a longer distance than others thereby accumulating a wavelength dependent delay. By proper design, the total dispersion of an optical link can be compensated in full. One disadvantage of this method is that it operates in reflection. Therefore it requires an additional photonic device to redirect the signal and prevent backscattering. Another disadvantage is that the dispersion spectrum is highly sensitive to 
inhomogeneities of the fiber. Small perturbation of the core refractive index along the propagation axis produces undesirable group delay ripple.

Fig. 1-8b shows that a chirped LPG can compensate dispersion as well. Because of the difference in refractive index, the group velocity of the cladding modes is larger than the group velocity of the core mode. A properly designed LPG forces faster wavelength components to propagate a larger distance in the core than slower components, thus the accumulated time delay can be reduced to zero. There is no need for an additional device in this case since there is no reflection as such. Since the LPG has a period much larger than the wavelength of the signal, it is less sensitive to waveguide inhomogeneities. However the total length of that compensator is larger than with a FBG.

\subsubsection{Sensors. [14], [15]}

The loss peak wavelengths of a LPG must satisfy the phase matching condition:

$$
\lambda=\left[n_{\text {eff }}(\lambda)-n_{\text {clad }}^{(m)}(\lambda)\right] \Lambda
$$

which is a function of the period of the $\operatorname{LPG}(\Lambda)$, of the order of the cladding mode to which the core mode couples $(m)$, and of the particular material of the fiber (through $n_{\text {eff }}, n_{\text {clad }}$ ). Therefore, the position of the rejection band on the wavelength axis can be affected by any environmental parameters through the influence on each of those three components.

\section{Temperature sensitivity.}

The temperature sensitivity can be found by evaluating the derivative of (1-5),

$$
\begin{aligned}
& \lambda=\lambda\left(\delta n_{\text {eff }}(T), \Lambda(T)\right) \\
& \frac{d \lambda}{d T}=\frac{\partial \lambda}{\partial\left(\delta n_{\text {eff }}\right)} \frac{d\left(\delta n_{\text {eff }}\right)}{d T}+\frac{\partial \lambda}{\partial \Lambda} \frac{d \Lambda}{d T} \\
& \frac{d \lambda}{d T}=\frac{\partial \lambda}{\partial\left(\delta n_{\text {eff }}\right)}\left(\frac{d n_{\text {eff }}}{d T}-\frac{d n_{\text {clad }}}{d T}\right)+\Lambda \frac{\partial \lambda}{\partial \Lambda} \frac{1}{L} \frac{d L}{d T}
\end{aligned}
$$


where $\delta n_{\text {eff }}=n_{\text {eff }}-n_{\text {clad }}, L$ is the length of LPG. Expression (1-6) can also be written in the form:

$\frac{d \lambda}{d T}=\Lambda\left(\frac{d n_{\text {eff }}}{d T}-\frac{d n_{\text {clad }}}{d T}\right)+\Lambda\left(n_{\text {eff }}-n_{\text {clad }}\right) \alpha$

where $\alpha=\frac{1}{L} \frac{d L}{d T}$.

The first term in equation (1-6) is related to the variation of difference between the effective indices of the core and cladding modes. Even though the geometry of the fiber plays the role in this effect, the main contribution is due to material parameters (fiber composition) and the order of the cladding mode. For coupling to the lower order cladding modes (by using $\Lambda>100 \mu \mathrm{m}$ ), the material parameters dominate [16]. For coupling to the higher order cladding modes (by using $\Lambda<100 \mu \mathrm{m}$ ), the material effects are negligible.

The second term is related to the variation of the resonance wavelength of a mode with respect to the LPG's period and it is strongly dependent on waveguide dispersion. The absolute value and sign of this term is strongly dependent on the cladding mode order, $m$. For low $m$, the derivative $\partial \lambda / \partial \Lambda$ is positive, and for high $m$ it is negative, see Fig. 1-9. By choosing the LPG's parameters, the temperature sensitivity can be balanced by both contributions, or be positive or negative. The same effect can be reached via careful choice of fiber materials. For telecom applications the temperature sensitivity must be negligible, while for monitoring the environment or thermally tuned filters the temperature sensitivity must be large. The rejection band shift in wavelength for different temperatures of LPG is shown in Fig. 1-10a, b. The wavelength shift is approximately linear with temperature in the range $30^{\circ} \mathrm{C}-160^{\circ} \mathrm{C}$. Temperature insensitive LPGs are those with period $<100 \mu \mathrm{m}$. In this case the core mode is coupled to the higher order cladding modes, which have low material contribution to the temperature sensitivity. By optimizing all the parameters: grating period, cladding mode and fiber components, the temperature sensitivity can as high as $275 \mathrm{~nm}$ per $100^{\circ} \mathrm{C}$. 

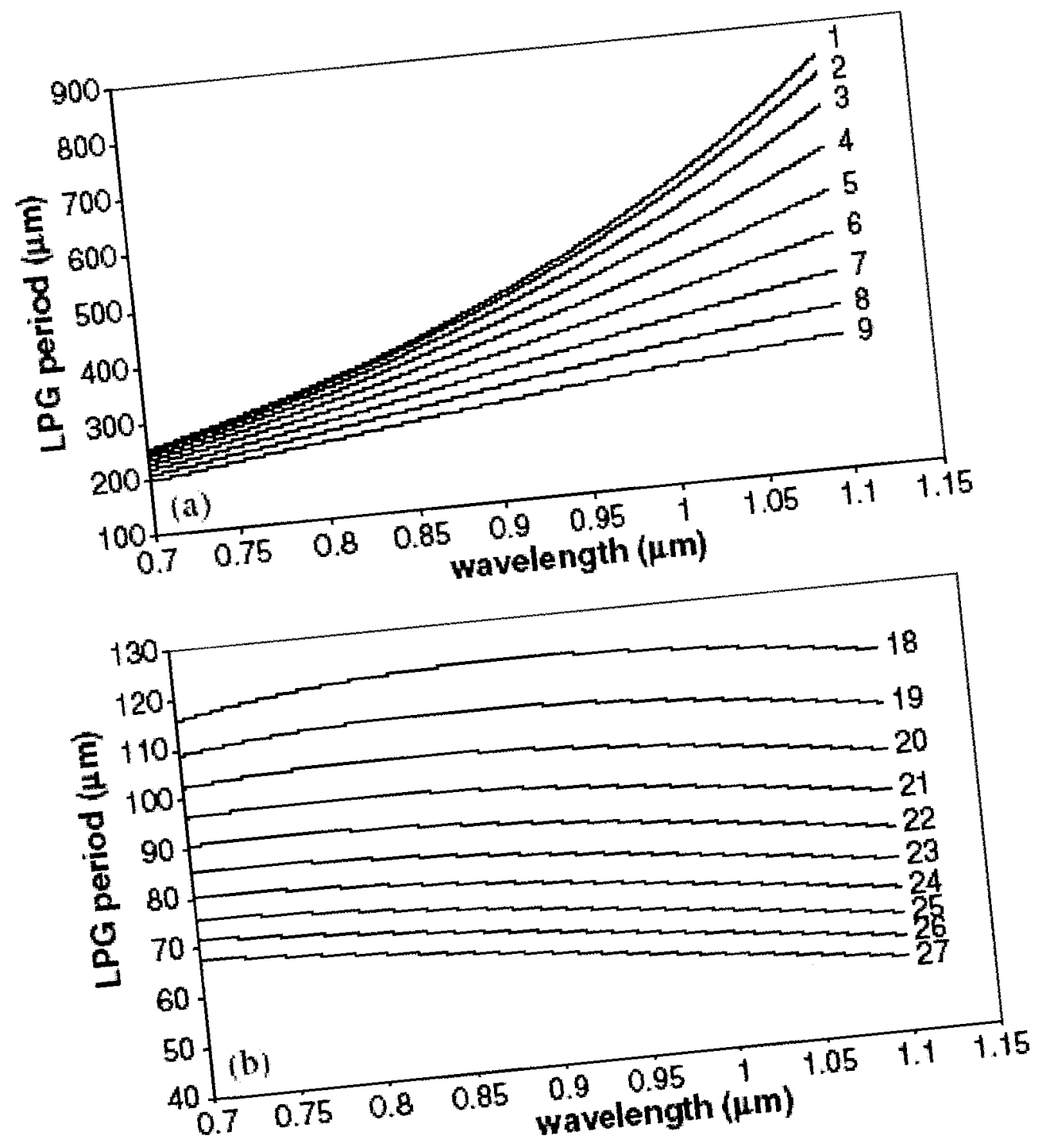

Fig. 1-9.
fiber. [14] 


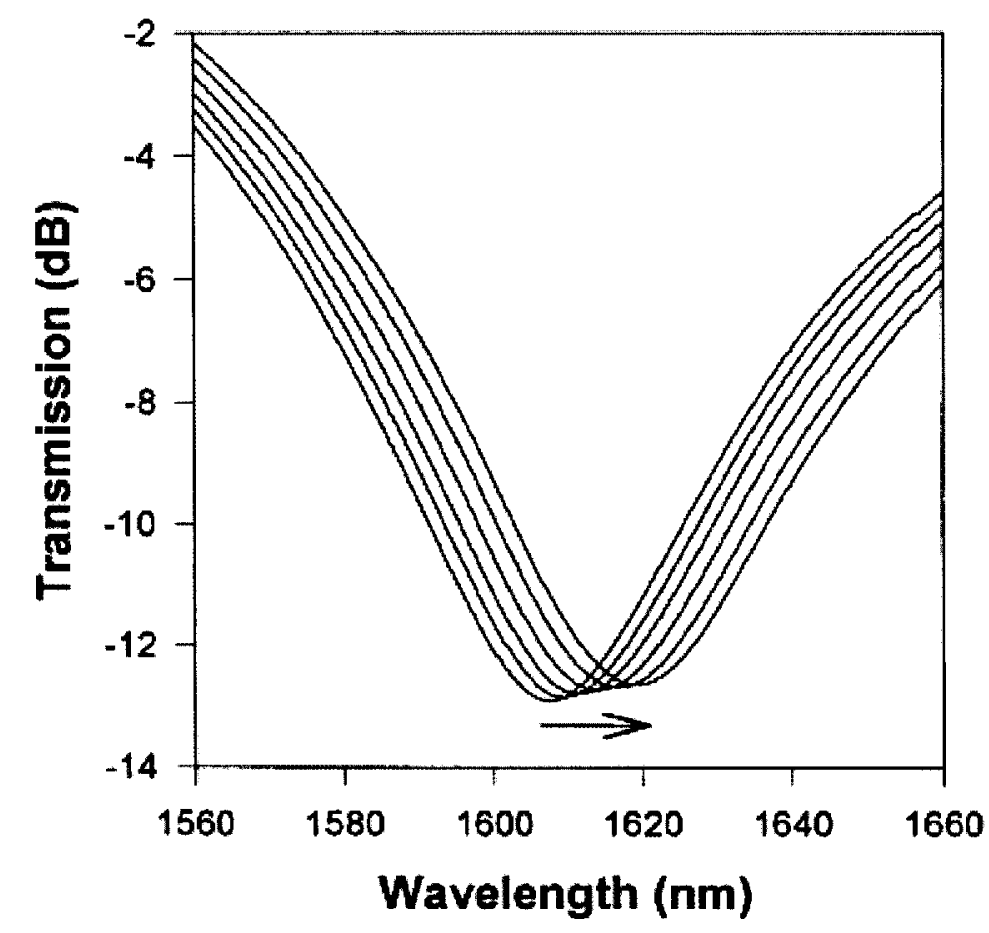

Fig. 1-10a. rejection band shift in wavelength for different values of LPG temperature $\left(22.7^{\circ} \mathrm{C}, 49.1^{\circ} \mathrm{C}, 74.0^{\circ} \mathrm{C}, 100.9^{\circ} \mathrm{C}, 127.3^{\circ} \mathrm{C}\right.$ and $149.7^{\circ} \mathrm{C}$ from left to right). a long-period grating with $\Lambda=280 \mu \mathrm{m}$ was fabricated in Corning SMF28. [15] 


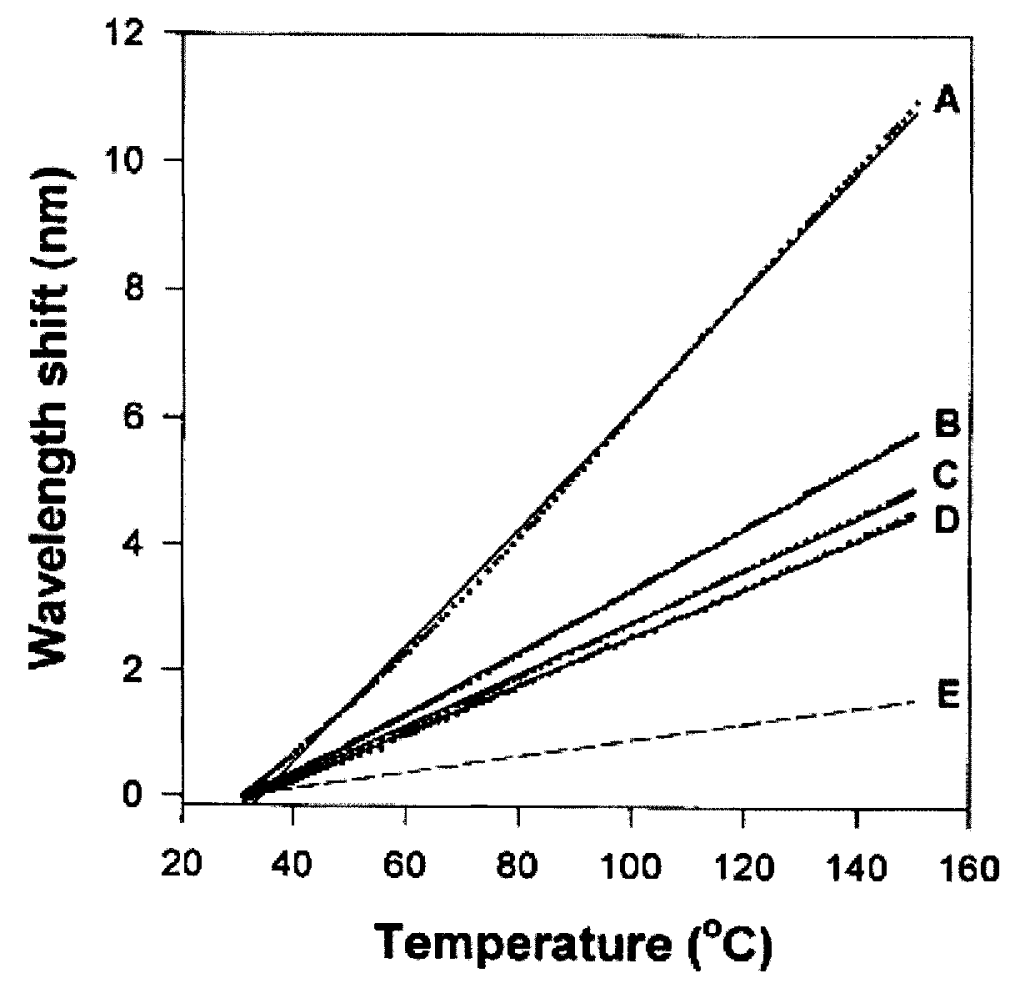

Fig. 1-10b. shift of the rejection peaks with temperature. [15]

\section{Strain sensitivity.}

The strain sensitivity along the propagation axis is evaluated in a similar fashion:

$\frac{d \lambda}{d \varepsilon}=\frac{\partial \lambda}{\partial\left(\delta n_{e f f}\right)}\left(\frac{d n_{e f f}}{d \varepsilon}-\frac{d n_{c l a d}}{d \varepsilon}\right)+\Lambda \frac{\partial \lambda}{\partial \Lambda}$

where $\Delta \varepsilon=\frac{\Delta L}{L}$. The first term is mainly due to material contributions and the second is due to waveguide contributions. For period $<100 \mu \mathrm{m}$ both contributions to the strain sensitivity are negative. For period $>100 \mu m$ they are of opposite sign thus reducing the sensitivity. Therefore, by choosing the period of the grating we can separate the response due to the temperature from the response due to the strain: when $\Lambda<100 \mu m$ LPG is temperature insensitive, when $\Lambda>100 \mu m$ LPG is strain insensitive. Typical value of LPG strain 
sensitivities written on Corning Flexcore fiber: $-2.2 \mathrm{pm} \mu \varepsilon^{-1}$ for $\Lambda=40 \mu \mathrm{m}$ and

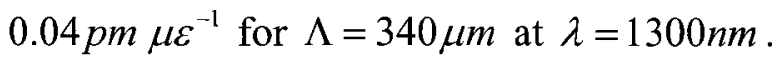

\section{External refractive sensitivity.}

The sensitivity of LPG to the external refractive index comes from the dependence of the phase matching condition upon the cladding mode effective index.

The cladding mode effective index depends not only on the actual cladding refractive index, $n_{\text {clad }}$ but on its surrounding refractive index, $n_{\text {sur }}$. Sensitivity to external index appears as a shift of central wavelength, i.e. wavelength for minimum transmission band, and as a change in attenuation. Plots of both quantities vs. external refractive index are given in Fig. 1-11. For $n_{\text {sur }}<n_{\text {clad }}$ the cladding mode is guided and its electric field concentrated mostly in the cladding region. In the ray optics approach this case is described by total internal reflection at the cladding-surrounding interface. In cases when $n_{\text {sur }} \approx n_{\text {clad }}$, cladding and surrounding are not distinguishable and the effective cladding has infinite extent. The cladding modes do not exist and rejection bands disappear. The sensitivity is the largest when this case is approached, as seen from the plots in Fig. 1-11. For $n_{\text {sur }}>n_{\text {clad }}$ the cladding mode is a leaky mode, the electric field radiates from the cladding region, and total internal reflection is replaced by complex valued Fresnel reflection. Refractive index sensitivity has to be taken into account in the process of packaging fiber LPG to ensure that adhesive materials do not change the central wavelength of the rejection band.

A refractive index sensor can be used to measure the concentration of some chemicals. For example, in binary fluid solutions of aromatic compound (xylene) in paraffin (heptane), the minimum detectible change in concentration is $0.04 \%$ corresponding to a refractive index change $\Delta n=6 \times 10^{-5}$. Such a sensor though is sensitive over limited range of indices only and is not able to distinguish two distinct elements with the same refractive index. 


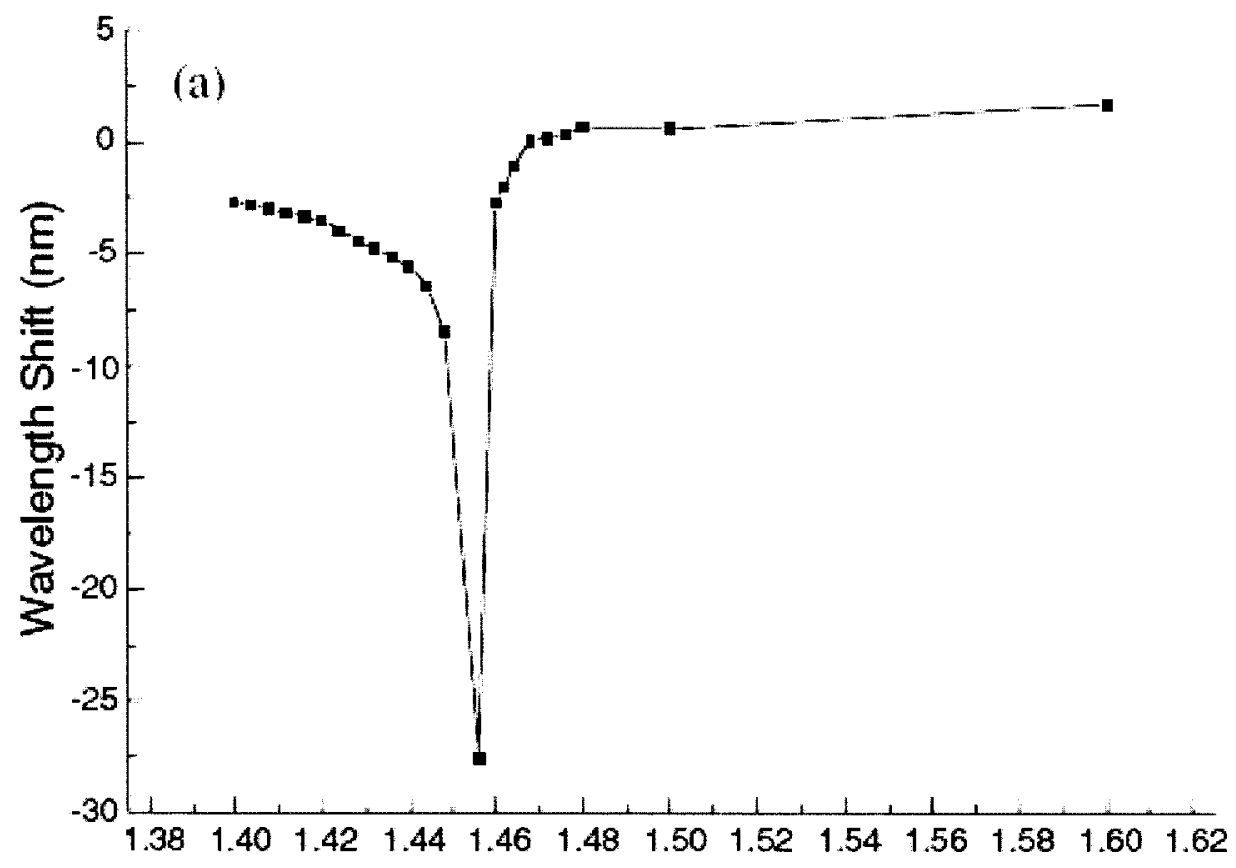

R.I.

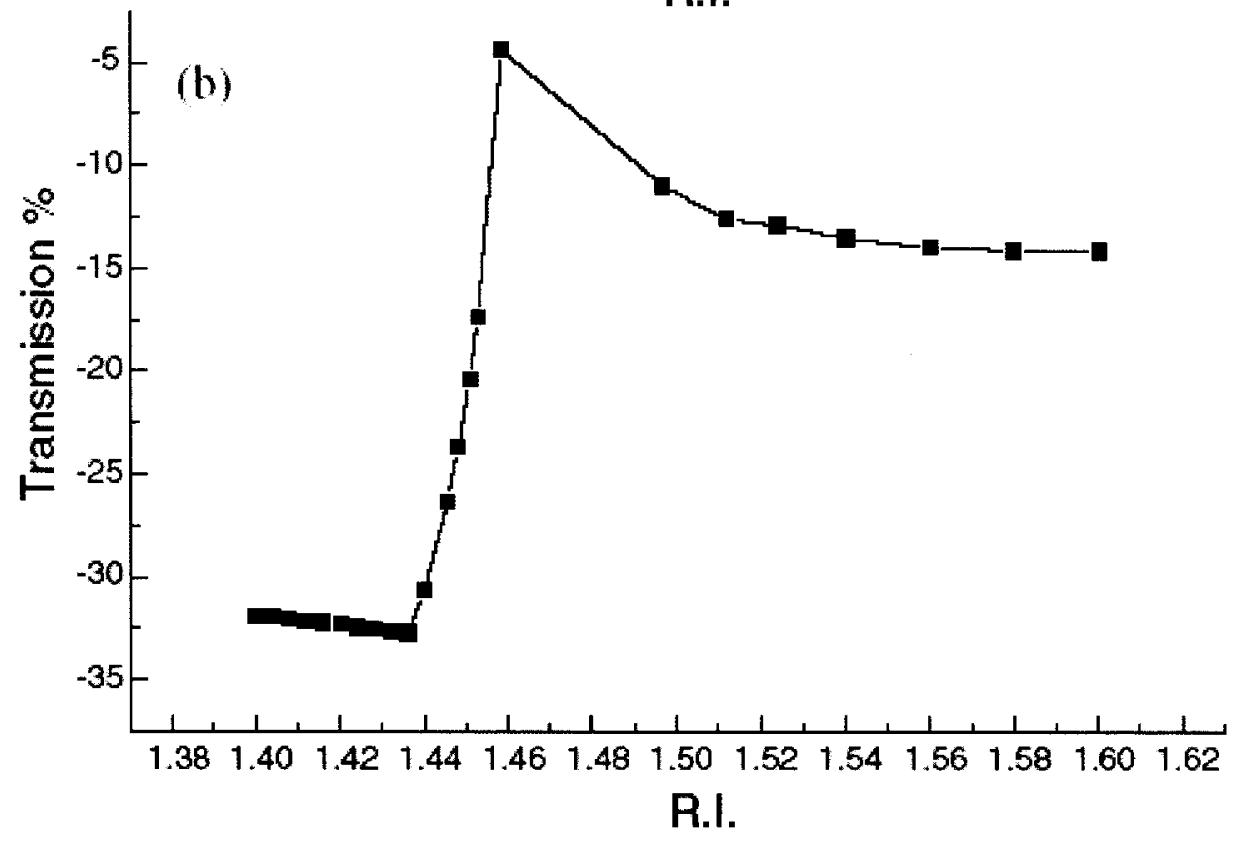

Fig. 1-11. Wavelength shift (a) and minimum transmission value of as a function of external refractive index. A period of grating is $400 \mu \mathrm{m}$, it is written in borongermanium co-doped fiber. [14] 
The sensitivity to external refractive index can modify the overall temperature sensitivity, i.e. when the LPG is surrounded by a material with large thermo-optic coefficient, then it will respond both to temperature change and to the refractive index change of the surrounding induced by temperature. When both effects shift the central wavelength in the same direction, the temperature response is increased. When they shift the central wavelength in opposite directions, the temperature response is decreased or even canceled.

For instance, surrounding the LPG with an acrylate-based polymer which has a negative thermo-optic coefficient and refractive index close to the cladding index, the temperature sensitivity increases from $5 \mathrm{~nm} / 100^{\circ} \mathrm{C}$ to $80 \mathrm{~nm} / 100^{\circ} \mathrm{C}$. Similarly, coating a LPG sensor with a material with a positive thermo-optic coefficient, the temperature sensitivity was reduced to $0.07 \mathrm{~nm} / 100^{\circ} \mathrm{C}$.

\section{Bend sensitivity.}

If a fiber LPG is bent, the rejection band shifts. Another possibility is the dual peak resonance, when a single rejection band is split in two distinct bands with a separation which depends on the bending radius. In the last case the symmetry of the system is broken and two degenerate cladding modes become non-degenerate. It is illustrated in Fig. 1-12. That effect is slightly different upon orientation of the fiber, this difference being attributed to radial non-uniform UV-exposure during grating fabrication.

As seen from Fig. 1-9 for some high-order cladding modes $(18,19)$ it is possible to couple the core mode to the cladding at two different wavelengths. Measuring the wavelength separation between the two peaks allows to increase the sensitivity of the bending measurement by 50 times over other methods.

\section{In series $L P G$ sensors.}

The arrangement of two LPGs in series can be viewed as a Mach-Zender interferometer, Fig. 1-13 describes its operation principle. Light passing in the fiber has two separate paths, one is direct through the core and another through the cladding by coupling twice in LPGs. The effective indices of the core mode and cladding modes are unequal and so are the optical 
paths. Therefore the phase difference accumulated at the end of the second LPG is non-zero resulting in an interference pattern. The transmission characteristics of two LPGs separated by 5 and $19 \mathrm{~cm}$ are shown in Fig. 1-14. Interference fringes are superimposed on top of the rejection band. The narrow band fringe pattern allows a greater measurement resolution. Such a sensor is used to sense bending, temperature and strain changes.

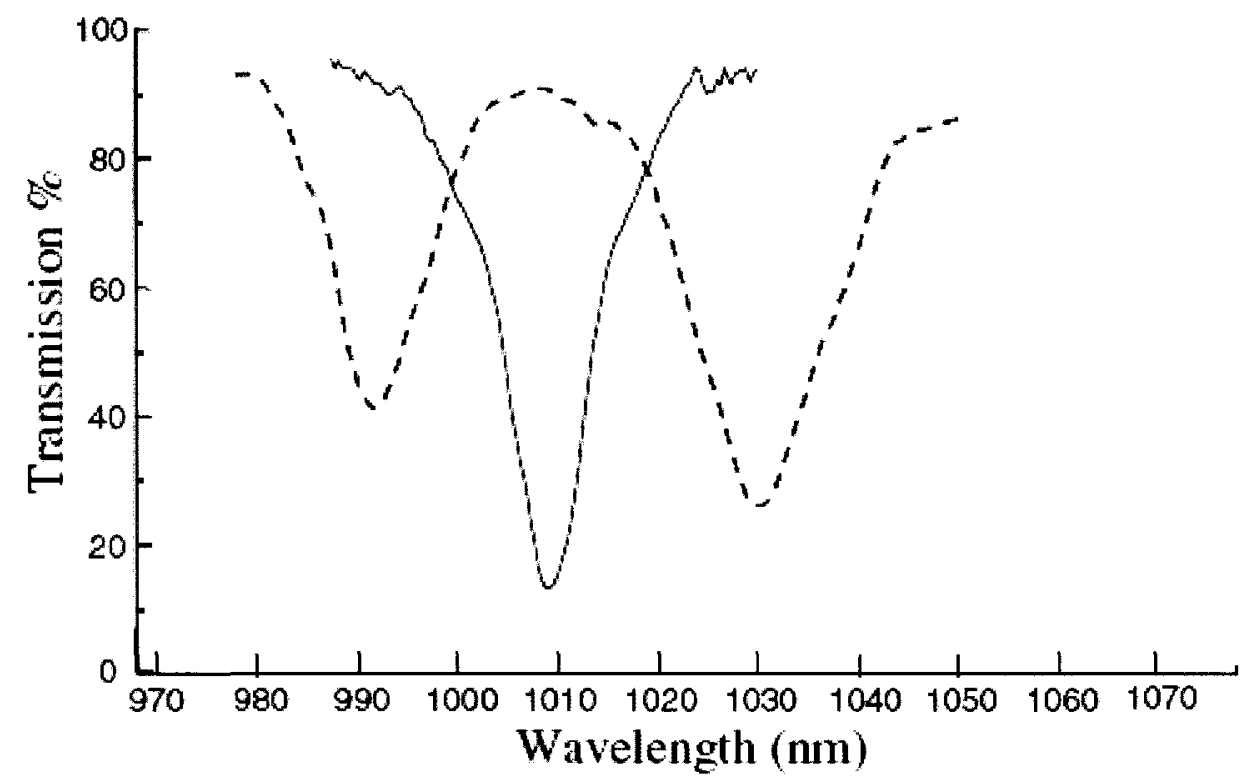

Fig. 1-12. Transmission characteristics of LPG fiber. Solid curve (single peak resonance): no bend. Dashed curve (dual peak resonance): bent curvature $1.55 \mathrm{~m}^{-1}$. Grating period is $400 \mu \mathrm{m}$, length is $40 \mathrm{~mm}$. [14]

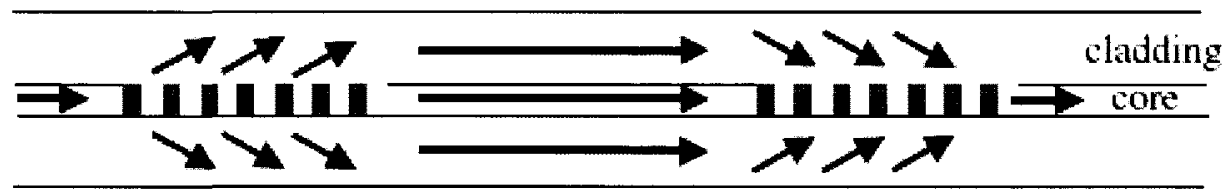

Fig. 1-13. Two LPGs in series acting as Mach-Zender interferometer. [14] 


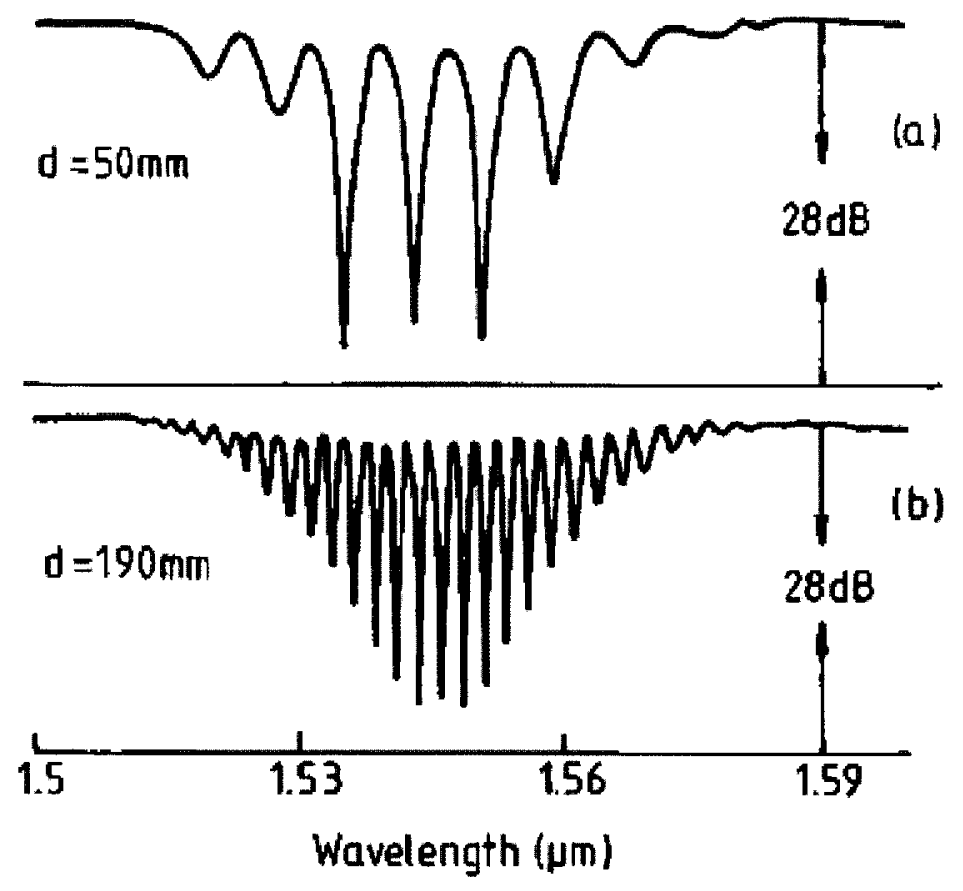

Fig. 1-14. Transmission characteristics of two LPGs in series. (a) separation is $50 \mathrm{~mm}$, (b) separation is $190 \mathrm{~mm}$. [14]

\subsection{Long period gratings in planar waveguides. [17]-[22]}

As has been shown in 1.2 long period gratings in fibers have proved to be very successful in many applications. However, the geometry and material constrains of the fiber result in functional limitations of the devices based on LPGs, especially active devices. Also, fiber is not suitable for integration. To overcome these limitations of the fiber, long-period waveguide gratings, LPWG have been proposed, [17]. Planar wave guides can be made with different shapes and sizes and different materials. This gives many more design patterns and hence an additional control over transmission characteristics. Several examples follow. An LPG in a 4-layer slab waveguide was analyzed in [18]. The spectrum of the LPWG is more sensitive to the polarization of light than in the case of fiber. However, with special choice of the geometrical parameters, a four-layer slab can be made with polarizationindependent rejection band characteristics. Also the thickness of the waveguide cladding, 
$d_{\text {clad }}$ can be made substantially smaller than in the case of fiber. This has an impact on the phase matching curves (resonance wavelength, $\lambda_{0}$ vs. grating period, $\Lambda$ ). For instance, a dual peak resonance (for a given $\Lambda$ two distinct $\lambda_{0}$ exist ) can occur even for the first order cladding mode, whereas in fiber it happens only for higher order modes.

Similarly with fibers by choosing an appropriate cladding material, the temperature sensitivity of the device can be controlled. A LPWG based on polymer-cladding ionexchanged BK7 glass was fabricated and experimentally analyzed, [19]. Due to a larger thermooptic effect of the polymer, the temperature sensitivity of the filter is almost $\sim 9.0 \mathrm{~nm} /{ }^{\circ} \mathrm{C}$, significantly higher than that in the fiber. This allows tuning the filter by over $180 \mathrm{~nm}$ with only $20^{\circ} \mathrm{C}$ of temperature change.

As discussed earlier, two parallel LPG in fibers can act as an add/drop filter, [12]. This idea can also be implemented and extended in planar waveguides, [20]. An array of six parallel single-mode channels with etched gratings was fabricated on one substrate. The separation between adjacent channels was $50 \mu \mathrm{m}$. Such wide separation prevents evanescent coupling between channels. When light is launched in one channel, it propagates along that channel and couples to all others only at the resonant wavelength. Qualitatively, light is coupled first to the cladding mode of the entire composite waveguide and then to each individual channel. This demonstration shows that a multi-channel add/drop filter having small cross-talk can be built based on LPG planar structure.

Finally, to make the transmission spectrum of the grating dynamically reconfigurable, the concept of temporarily induced grating was introduced, [21]. The grating is induced by the thermooptic effect. Such thermoptically induced grating later was experimentally implemented and analyzed, [22]. The notch filter has a single mode channel, a cladding and two buffer layers surrounding the cladding. Periodic heaters are formed in the upper buffer with period equal to desired LPG. By changing the applied power to the heaters the transmission characteristics of the device can be controlled in two ways: first by modulating the overall attenuation and second, by shifting the resonance wavelength in a desired direction. The required power is of the order of $1 \mathrm{~W}$. By applying power to the heater with a 
square-wave impulse, the form time response of the filter was measured, i.e. optical intensity of the core mode. Rising and falling edges of the optical pulses were around $\sim 40 \mathrm{msec}$.

1.4 Non-linear effects. (followed by [23], [24], [25])

The refractive index of optical materials changes in the presence of intense electric fields is given by (see Appendix F):

$n_{\text {eff }} \approx n_{0}+n_{2}\left|E_{0}\right|^{2}$

This is known as the optical Kerr effect, where $\vec{E}_{0}$ is the electric field vector of an intense light beam, $n_{0}=\sqrt{1+\chi_{\omega}{ }^{(1)}}$ is the linear (low intensity) component of the refractive index, $n_{2}=\frac{3}{8} \chi_{\omega}^{(3)} \frac{1}{n_{0}}$ is the Kerr coefficient, $\chi^{(1)}$ is the linear susceptibility and $\chi^{(3)}$ is the nonlinear third order susceptibility of the media. For fused silica $n_{2}$ has the value $0.6 \times 10^{-22}\left[\frac{\mathrm{V}}{\mathrm{m}}\right]^{-2}, n_{0}=1.46$. The relation between the optical power density in the fiber, $P$ and the electric field strength, $E$ can be approximated as: $P=\frac{n_{0}|E|^{2}}{2 Z_{0}}$, where $Z_{0}=\sqrt{\frac{\mu_{0}}{\varepsilon_{0}}} \approx 377[\Omega]$ is the impedance of free space. Then for a typical effective core area of the fiber, $\mathrm{A}=50[\mu \mathrm{m}]^{2}$ and optical power of $1[\mathrm{~W}]$, the power density is $P=\frac{1[\mathrm{~W}]}{50[\mu \mathrm{m}]^{2}}=2 \times 10^{10}\left[\frac{\mathrm{W}}{\mathrm{m}^{2}}\right]$ and the intensity-dependent component of the refractive index is: $n_{2}|E|^{2}=n_{2} \frac{2 P Z_{0}}{n_{0}}=0.6 \times 10^{-22} \frac{2 \times 2 \times 10^{10} \times 377}{1.46} \approx 0.62 \times 10^{-9}$. The optical Kerr effect is associated with an atomic transition from the ground state to a virtual state: the life time of such state is relatively short, $\sim 10^{-13}-10^{-14}[\mathrm{sec}]$. So, the modulation of the refractive index $(\Delta n)$ in the fiber due to the Kerr effect is small but very fast. In order to increase the modulation $\Delta n$, we can dope the fiber with rare earth elements, such as $E r, Y b$. They have 
strong absorption bands: $E r$ at wavelengths near $\lambda=1520-1560[\mathrm{~nm}]$ and $Y b$ at wavelengths near $\lambda=980[\mathrm{~nm}]$. A pump beam tuned to such bands strongly modifies the population of the ground state and therefore the refractive index of the doped fiber. Now atomic transitions (absorption) are associated with actual states rather than virtual. Therefore, the life time of the excited state is of the order of $\sim 1-10[n s]$. By small detuning of the pump from the actual transition, unwanted absorption is reduced, while the nonlinear refractive index change remains considerable. Theory predicts that such enhancement of nonlinear index due to a resonant dopant can be stronger by $10^{9}$ times over the pure intrinsic Kerr effect. In the laboratory, an experimental improvement factor of 140000 has been demonstrated, [25]. However, the response time of such modulation is much slower. In this last example, we evaluate the refractive index modulation as:

$$
\Delta n=140000 \times n_{2}|E|^{2}=140000 \times 0.62 \times 10^{-9} \approx 0.87 \times 10^{-4} \text {. }
$$

Based on this result, since we intend to propose using nonlinear effect to switch our LPWG, we will conservatively set the available $\Delta n$ to $10^{-6}$ to evaluate our designs.

\subsection{Goals of this work}

From the literature review we have seen that long period fiber gratings are sensitive to environmental changes including external refractive index variations. An additional layer deposited on the cladding with a higher refractive index than the cladding, increases sensitivity even further, [26], [27]. We have also seen that a long period grating in a planar waveguide offers more functionality and better performance due to an additional design parameters. That is the motivation. Our goal is to investigate planar waveguides with two guiding channels and a long period grating used to couple energy between the channels, and to investigate its sensitivity to internal refractive index changes due to nonlinear effects. Two guiding channels imitate a fiber with an additional coating layer. We hope, that the combination of two factors, an additional layer and a planar waveguide structure, will improve the sensitivity characteristics. More complicated structures, like three guiding channels, may be even better but we want to start with the simplest model possible. If we 
succeed and the device shows high sensitivity, let's say to index changes of the order $\sim 10^{-6}$, that would also mean the device is suitable for switching through the nonlinear effects controlled by relatively low optical power. We are also interested to know how fast that switching time might be. If it is relatively slow, it might be useful in areas where switching speed is not a concern, for example reconfigurable DWDM system. If switching is fast, it might be useful in a laser cavity as a Q-switch or even as a fast saturable absorber in laser modelocking. We also want to know the physical length of potential devices. For example, a compact optical switch can find an application in integrated optics, while an excessively long device is useless everywhere. In order to optimize the spectral characteristics of the device we need to know the influence of various geometrical and material parameters. In order to solve the five-layer slab problem, we have access to a commercial software package named $\mathrm{C} 2 \mathrm{~V}$ and will do simulations and optimizations numerically. However it is always nice to find a closed form analytical solution for the problem. When a function is known analytically, optimal values can be found faster and more accurately. To estimate an analytical solution we will use perturbation theory applied to a related system with known analytical solutions. 


\section{Chapter 2 \\ Coupled mode theory and its applications}

In this chapter we derive the transfer matrix and transmission function of a codirectional coupler which will be used later for calculating the spectral characteristics of our device. One standard approach of finding the transmission function of a coupler is by applying a coupled mode theory, a powerful tool to analyze various optical devices. We present it here and its applications to contradirectional and codirectional couplers which can be implemented with Bragg gratings and long period gratings respectively.

\subsection{Coupled mode theory (CMT)}

Coupled mode theory was analyzed in textbooks, see for example [28], [29], [30] and article [31]. We outline here the main steps of coupled mode theory and focus our attention on assumptions made.

Assume that a waveguide is homogeneous along its z-axis, i.e. its permittivity $\boldsymbol{\varepsilon}(x, y)$ does not depend on $\mathrm{z}$ but may depend on $\mathrm{x}$ and $\mathrm{y}$. Then its modes are orthogonal to each other,

$$
\iint_{x, y} E_{k} \cdot E_{m}^{*} \cdot d x d y=\left\{\begin{array}{ccc}
\neq 0 & \text { if } & m=k \\
0 & \text { if } & m \neq k
\end{array}\right.
$$

We assumed that modes are orthogonal but not necessary normalized to unit power. The electric field of each mode has the form

$E_{m}(x, y) \exp \left[j\left(\omega t-\beta_{m} z\right)\right]$

An arbitrary electric field in the waveguide is a linear superposition of modal fields,

$$
E=\sum_{m}\left\{A_{m} E_{m}(x, y) \cdot \exp \left[j\left(\omega t-\beta_{m} z\right)\right]\right\}
$$


The coefficients $A_{m}$ do not depend on $z$. This means that the contribution of each mode to the field, launched in the waveguide, stays the same during its propagation. $A_{m}$ represents a mode field propagating in the forward direction.

The electric field satisfies the wave equation:

$$
\left[\frac{\partial^{2}}{\partial x^{2}}+\frac{\partial^{2}}{\partial y^{2}}+\frac{\partial^{2}}{\partial z^{2}}+\omega^{2} \mu \varepsilon(x, y)\right] \cdot E=0
$$

Each of the modal fields also satisfies the wave equation,

$$
\left[\frac{\partial^{2}}{\partial x^{2}}+\frac{\partial^{2}}{\partial y^{2}}+\omega^{2} \mu \varepsilon(x, y)-\beta_{m}^{2}\right] \cdot E_{m}=0
$$

Now an inhomogeneous disturbance along the z-axis is introduced as a small perturbation to the waveguide, so the permittivity of the perturbed waveguide can be written in the form, $\varepsilon_{\text {per }}(x, y, z)=\varepsilon(x, y)+\Delta \varepsilon(x, y, z)$.

The permittivity disturbance $\Delta \varepsilon$ generates a polarization disturbance $\Delta P$ as seen from the constitutive relation:

$$
\begin{aligned}
& D=\varepsilon E=\varepsilon_{0} E+P \\
& D_{p e r}=\varepsilon_{p e r} E=(\varepsilon+\Delta \varepsilon) E=\varepsilon E+\Delta \varepsilon E=D+\Delta P \\
& \Delta P=\Delta \varepsilon \cdot E
\end{aligned}
$$

the presence of a polarization disturbance $\Delta P$ modifies the wave equation:

$$
\left[\frac{\partial^{2}}{\partial x^{2}}+\frac{\partial^{2}}{\partial y^{2}}+\frac{\partial^{2}}{\partial z^{2}}+\omega^{2} \mu \varepsilon(x, y)\right] \cdot E=-\omega^{2} \mu \cdot \Delta P
$$

Where $-\omega^{2} \mu \cdot \Delta P$ is acting as a source term. The wave equations for different modes are coupled now through the polarization disturbance $\Delta P$ and this leads to the energy exchange between different modes. They are no longer orthogonal. It also means that if an arbitrary field propagates in the waveguide it can still be viewed as a superposition of the modal fields, however the contribution of each mode now varies with propagation, i.e. coefficients $A_{m}$ are no longer constant, they become z-dependent. 


$$
E=\sum_{m}\left\{A_{m}(z) \cdot E_{m}(x, y) \cdot \exp \left[j\left(\omega t-\beta_{m} z\right)\right]\right\}
$$

where $\beta_{m}$ is a propagation constant, it is positive for a forward mode and negative for a backward mode. Substitution (2-10) into wave equation (2-9) gives:

$$
\sum_{m}\left[\frac{d^{2} A_{m}}{d z^{2}}-j 2 \beta_{m} \frac{d A_{m}}{d z}\right] \cdot E_{m}(x, y) \cdot \exp \left[j\left(\omega t-\beta_{m} z\right)\right]=-\omega^{2} \mu \cdot \Delta P
$$

where we have used equation (2-4).

With a slowly varying envelope approximation:

$$
\left|\frac{d^{2} A_{m}}{d z^{2}}\right| \ll<\left|2 \beta_{m} \frac{d A_{m}}{d z}\right| \text {, }
$$

equation (2-11) reduces to

$$
\sum_{m} j 2 \beta_{m} \frac{d A_{m}}{d z} \cdot E_{m}(x, y) \cdot \exp \left[j\left(\omega t-\beta_{m} z\right)\right]=\omega^{2} \mu \cdot \Delta P
$$

Now we use the orthogonality property of unperturbed modes, i.e. we multiply (2-13) by $E_{m}^{*}$ and integrate over the $x-y$ plane.

$$
j 2 \beta_{m} \frac{d A_{m}}{d z} \exp \left[j\left(\omega t-\beta_{m} z\right)\right] \cdot \int_{-\infty}^{\infty} \int_{-\infty}^{\infty} E_{m} \cdot E_{m}^{*} \cdot d x d y=\omega^{2} \mu \cdot \int_{-\infty}^{\infty} \int_{-\infty}^{\infty} \Delta P \cdot E_{m}^{*} \cdot d x d y
$$

inserting (2-8) and (2-10)

$$
\begin{gathered}
j 2 \beta_{m} \frac{d A_{m}}{d z} \exp \left[-j \beta_{m} z\right] \int_{-\infty}^{\infty} \int_{-\infty}^{\infty} E_{m} E_{m}^{*} d x d y=\omega^{2} \mu \sum_{l} A_{l} \exp \left[-j \beta_{l} z\right] \int_{-\infty}^{\infty} \int_{-\infty}^{\infty} E_{l} \Delta \varepsilon E_{m}^{*} d x d y \\
\ldots(2-15) \\
\frac{d A_{m}}{d z}=-j \frac{\omega^{2} \mu}{2 \beta_{m}} \frac{1}{\int_{-\infty}^{\infty} \int_{-\infty}^{\infty} E_{m} E_{m}^{*} d x d y} \sum_{l} A_{l} \exp \left[-j\left(\beta_{l}-\beta_{m}\right) z\right] \int_{-\infty}^{\infty} \int_{-\infty}^{\infty} E_{l} \Delta \varepsilon E_{m}^{*} d x d y
\end{gathered}
$$


$\frac{d A_{m}}{d z}=-j \frac{\left|\beta_{m}\right|}{\beta_{m}} \sum_{l} A_{l} \cdot C_{l m} \exp \left[j\left(\beta_{m}-\beta_{l}\right) z\right]$

where $C_{l m}=\frac{\omega^{2} \mu}{2\left|\beta_{m}\right|} \frac{\int_{-\infty}^{\infty} \int_{-\infty}^{\infty} E_{l} \Delta \varepsilon E_{m}^{*} d x d y}{\int_{-\infty}^{\infty} \int_{-\infty}^{\infty} E_{m} E_{m}^{*} d x d y}$

is the coupling coefficient between modes $l$ and $m$.

(2-17) is the system of coupled mode equations between different modes in the waveguide.

Now we look closer under what condition it is valid. The unperturbed waveguide was inhomogeneous along the $\mathrm{x}$ and $\mathrm{y}$ axes, $\varepsilon(x, y)$. Under this condition wave equations (2-3) and (2-4) are not exact, we have neglected term involving $\nabla \varepsilon$. We have used a perturbation approach, which implies that perturbation disturbance is relatively small and does not modify significantly the modes of the waveguide. Otherwise the expansion (2-10) is not valid (even though coefficients $A_{m}(z)$ are z-dependent). Then we have used the slow amplitude approximation, ( [30] p.182):

$$
\left|\frac{d^{2} A_{m}}{d z^{2}}\right| \ll\left|2 \beta_{m} \frac{d A_{m}}{d z}\right|
$$

which can be simplified as:

$\left|\frac{d f}{d z}\right| \ll\left|2 \beta_{m} f\right|,\left|\frac{d f}{d z}\right| \ll\left|\frac{4 \pi}{\lambda} n_{e f f}^{(m)} f\right|,\left|\frac{d f}{f}\right| \ll\left|\frac{4 \pi d z}{\lambda} n_{e f f}^{(m)}\right|,\left|\frac{d f}{f}\right| \ll\left|4 \pi \cdot n_{e f f}^{(m)}\right|$ for $d z \approx \lambda$ where $f=\frac{d A_{m}}{d z}$

The meaning of that approximation is that the relative change of first derivative, $\frac{d A_{m}}{d z}$ over the distance of one wavelength should much less than $4 \pi \cdot n_{\text {eff }}^{(m)}$. We have assumed a TE mode only. To summarize, the assumptions are:

1. $\nabla \varepsilon(x, y)<<1$

2. $\Delta \varepsilon(x, y, z)<<\varepsilon(x, y)$ 
3. $\left|\frac{d f}{f}\right| \ll\left|4 \pi \cdot n_{e f f}^{(m)}\right|, f=\frac{d A_{m}}{d z}$

4. TE mode

We restrict our perturbation disturbance to a sinusoidal function with period $\Lambda$,

$$
\Delta \varepsilon(x, y, z)=\varepsilon_{p}(x, y) \cdot \cos \left(j \frac{2 \pi}{\Lambda} z\right)=\varepsilon_{p} \frac{1}{2}\left\{\exp \left[j \frac{2 \pi}{\Lambda} z\right]+\exp \left[-j \frac{2 \pi}{\Lambda} z\right]\right\}
$$

If $\Delta \varepsilon(x, y, z)$ is an arbitrary periodic function, then a Fourier series expansion has to be used. Then system (2-17) becomes:

$$
\frac{d A_{m}}{d z}=-j \frac{\left|\beta_{m}\right|}{\beta_{m}}\left\{A_{m} \cdot C_{m m}+\sum_{l, l \neq m} A_{l} \cdot C_{l m} \exp \left[j\left(\beta_{m}-\beta_{l}\right) z\right]\right\}
$$

A periodic perturbation is applied to a certain portion of the waveguide cross-section $S_{P E R}$, therefore the corresponding overlap integral should be taken over that portion only,

$$
\begin{aligned}
& C_{l m}=\frac{1}{2}\left\{\exp \left[j \frac{2 \pi}{\Lambda} z\right]+\exp \left[-j \frac{2 \pi}{\Lambda} z\right]\right\} \frac{\omega^{2} \mu}{2\left|\beta_{m}\right|} \frac{\iint_{P E R} E_{l} \cdot \varepsilon_{p} \cdot E_{m}^{*} \cdot d s}{\int_{-\infty}^{\infty} \int_{-\infty}^{\infty} E_{m} E_{m}^{*} d x d y}= \\
& =\left\{\exp \left[j \frac{2 \pi}{\Lambda} z\right]+\exp \left[-j \frac{2 \pi}{\Lambda} z\right]\right\} D_{l m} \\
& D_{l m}=\frac{\omega^{2} \mu}{4\left|\beta_{m}\right|} \iint_{S_{P E R}} E_{l} \cdot \varepsilon_{p} \cdot E_{m}^{*} \cdot d s \\
& \int_{-\infty}^{\infty} \int_{-\infty}^{\infty} E_{m} E_{m}^{*} d x d y
\end{aligned}
$$

In addition we will use one more approximation, so called phase matching. We keep only those terms that have similar spatial frequency. This approximation can be viewed as a continuation of the slowly wave approximation. The LHS has very low frequency. The first term of the RHS of (2-21) varies along z with frequency $f_{Z}=j \frac{2 \pi}{\Lambda}$ due to factor $C_{m m}$, while 
the second term varies with frequency $f_{Z}=\beta_{m}-\beta_{l}-\frac{2 \pi}{\Lambda}$ due to factor

$C_{l m} \exp \left[j\left(\beta_{m}-\beta_{l}\right) z\right]$. At the resonance condition, the second term oscillates at a zero-

frequency: $f_{Z}=\beta_{m}-\beta_{l}-\frac{2 \pi}{\Lambda}=0$. Assuming that for a given period $\Lambda$ the resonance condition (phase matching condition) is satisfied for mode $m$ and mode $l$ only, all other terms in the sum will have non-zero spatial frequencies because they are far from the resonance. Therefore we will keep only the LHS and one component of the second term of the RHS in our final equation:

$$
\begin{aligned}
& \frac{d A_{m}}{d z}=-j \frac{\left|\beta_{m}\right|}{\beta_{m}}\left\{A_{l} \cdot C_{l m} \exp \left[j\left(\beta_{m}-\beta_{l}\right) z\right]\right\}= \\
& =-j \frac{\left|\beta_{m}\right|}{\beta_{m}}\left\{A_{l} \cdot D_{l m} \exp \left[j\left(\beta_{m}-\beta_{l}-\frac{2 \pi}{\Lambda}\right) z\right]\right\}
\end{aligned}
$$

In a similar fashion we can write equation for mode $l$ :

$$
\frac{d A_{l}}{d z}=-j \frac{\left|\beta_{l}\right|}{\beta_{l}}\left\{A_{m} \cdot D_{m l} \exp \left[-j\left(\beta_{m}-\beta_{l}-\frac{2 \pi}{\Lambda}\right) z\right]\right\}
$$

One more assumption we did so far is a non-degeneracy, i.e. that each mode has a distinct electric field distribution $E_{m}(x, y)$. However, there is a situation when two different modes have the same electric field distribution. This happens when one mode propagates forward with propagation constant $+\left|\beta_{m}\right|$ and another mode propagates backward with propagation constant $-\left|\beta_{m}\right|$. Equations (2-24), (2-25) cover coupling between two modes for the following cases:

- mode $m$ and mode $l$ are propagating in the same direction (and their field distribution are distinct)

- mode $m$ and mode $l$ are propagating in the opposite direction (and their field distribution are distinct)

One more case is left to cover when mode $m$ and mode $l$ are propagating in the opposite direction and their field distribution are identical, i.e. case when $l=-m$. 
Assuming that the waveguide can support simultaneously forward and backward modes including degenerate case, the general electric field can be expressed as:

$$
E=\sum_{m}\left\{A_{m} E_{m}(x, y) \cdot \exp \left[j\left(\omega t-\beta_{m} z\right)\right]+B_{-m} E_{m}(x, y) \cdot \exp \left[j\left(\omega t+\beta_{m} z\right)\right]\right\}
$$

where $A_{m}$ represents a forward mode and $B_{-m}$ represents a backward mode. We can repeat all the steps above from (2-1) to (2-17). In this case the coupled mode equation (analogue to (2-17)) becomes:

$$
\frac{d A_{m}}{d z} \exp \left[-j \beta_{m} z\right]-\frac{d B_{-m}}{d z} \exp \left[j \beta_{m} z\right]=-j \sum_{l} C_{l m}\left\{A_{l} \exp \left[-j \beta_{l} z\right]+B_{-l} \exp \left[j \beta_{l} z\right]\right\}
$$

where two terms now remain on the LHS of (2-27). Coupling coefficient $C_{l m}$ is defined as:

$$
C_{l m}=\frac{\omega^{2} \mu}{2 \beta_{m}} \frac{\int_{-\infty}^{\infty} \int_{-\infty}^{\infty} E_{l} \Delta \varepsilon E_{m}^{*} d x d y}{\int_{-\infty}^{\infty} \int_{-\infty}^{\infty} E_{m} E_{m}^{*} d x d y}
$$

Again, we consider coupling between two modes only, $A_{m}$ and $B_{-m}$. Coupling between other modes is ignored because the phase matching condition for them is not satisfied.

$$
\begin{aligned}
& \frac{d A_{m}}{d z} \exp \left[-j \beta_{m} z\right]-\frac{d B_{-m}}{d z} \exp \left[j \beta_{m} z\right]= \\
& =-j C_{m m}\left(A_{m} \exp \left[-j \beta_{m} z\right]+B_{-m} \exp \left[j \beta_{m} z\right]\right)-j \sum_{l, l \neq m} C_{m l}\left(A_{l} \exp \left[-j \beta_{m} z\right]+B_{-l} \exp \left[j \beta_{l} z\right]\right)
\end{aligned}
$$

For $l \neq m$ the phase matching condition is not satisfied, $\pm \beta_{m} \pm \beta_{l} \pm \frac{2 \pi}{\Lambda} \neq 0$, then two terms only remain in the RHS,

$$
\frac{d A_{m}}{d z} \exp \left[-j \beta_{m} z\right]-\frac{d B_{-m}}{d z} \exp \left[j \beta_{m} z\right]=-j C_{m m}\left(A_{l} \exp \left[-j \beta_{m} z\right]+B_{-m} \exp \left[j \beta_{m} z\right]\right)
$$


And this equation can be separated into two independent equations because again of phase matching. Now we want to identify terms with similar spatial frequencies near the resonance conditions,

$$
\begin{array}{ll}
\frac{d A_{m}}{d z} \exp \left[-j \beta_{m} z\right]: & f_{Z}=-\beta_{m} \\
\frac{d B_{-m}}{d z} \exp \left[j \beta_{m} z\right]: & f_{Z}=\beta_{m}
\end{array}
$$

Phase matching with $\frac{d A_{m}}{d z} \exp \left[-j \beta_{m} z\right]$ requires $f_{Z}^{R H S}=-\beta_{m}=\beta_{m} \pm \frac{2 \pi}{\Lambda}$

$$
\begin{aligned}
& C_{m m} A_{m} \exp \left[-j \beta_{m} z\right]: \quad f_{Z}=-\beta_{m} \pm \frac{2 \pi}{\Lambda} \rightarrow \beta_{m} \quad \text { if } \quad \frac{2 \pi}{\Lambda}=2 \beta_{m} \\
& C_{m m} B_{-m} \exp \left[j \beta_{m} z\right]: \quad f_{Z}=\beta_{m} \pm \frac{2 \pi}{\Lambda} \rightarrow-\beta_{m} \quad \text { if } \quad \frac{2 \pi}{\Lambda}=-2 \beta_{m}
\end{aligned}
$$

Therefore by using phase matching we can break equation (2-28) into two:

$$
\begin{aligned}
& \frac{d A_{m}}{d z} \exp \left[-j \beta_{m} z\right]=-j C_{m m} B_{-m} \exp \left[j \beta_{m} z\right] \\
& \frac{d B_{-m}}{d z} \exp \left[j \beta_{m} z\right]=j C_{m m} A_{m} \exp \left[-j \beta_{m} z\right] \\
& \frac{d A_{m}}{d z}=-j D_{m m} B_{-m} \exp \left[j\left(2 \beta_{m}-\frac{2 \pi}{\Lambda}\right) z\right] \\
& \frac{d B_{-m}}{d z}=j D_{m m} A_{m} \exp \left[-j\left(2 \beta_{m}-\frac{2 \pi}{\Lambda}\right) z\right]
\end{aligned}
$$

The coupled mode equations (2-26) describe codirectional and contradirectional coupling between two modes with distinct field distribution $(l, m \quad l \neq m, \quad l \neq-m)$.Equations (2-31) describe contradirectional coupling between two modes with identical field distribution.

\subsection{Contradirectional coupling}

Contradirectional coupling between two modes, propagating forward, $A_{m}$ and backward, $B_{-m}$ is described by equations (2-31). 
$\frac{d A_{m}}{d z}=B_{-m} q^{*} \exp [-j 2 \delta z]$

$\frac{d B_{-m}}{d z}=A_{m} q \exp [j 2 \delta z]$

where $q^{*}=-j D_{m m}, q=j D_{m m}, \delta=\frac{\pi}{\Lambda}-\beta_{m}$.

With the substitution,

$b_{F}=A_{m} \exp [j \delta z], b_{B}=B_{-m} \exp [-j \delta z]$ the above equations transformed in the form,

$\frac{d b_{F}}{d z}=j \delta b_{F}+q^{*} b_{B}$

$\frac{d b_{B}}{d z}=-j \delta b_{B}+q b_{F}$

$\Delta \varepsilon=\varepsilon_{0} \Delta \varepsilon_{r}=\varepsilon_{0}\left(n_{p e r}^{2}-n^{2}\right)=\varepsilon_{0}\left((n+\Delta n)^{2}-n^{2}\right) \approx \varepsilon_{0} 2 n \Delta n=\varepsilon_{0} 2 n \Delta n_{0} \cos \left(\frac{2 \pi}{\Lambda} z\right)$

$\Delta \varepsilon=\varepsilon_{p} \cos \left(\frac{2 \pi}{\Lambda} z\right)$

$\varepsilon_{p}=\varepsilon_{0} 2 n \Delta n_{0}$

A periodic perturbation is applied to a certain portion of the waveguide cross-section, $S_{P E R}$ :

$$
\begin{aligned}
& D_{m m}=\frac{\omega^{2} \mu}{4 \beta_{m}} \frac{\iint_{S_{P E R}} E_{m} \cdot \varepsilon_{p} \cdot E_{m}^{*} \cdot d s}{\iint_{-\infty}^{\infty} \int_{-\infty}^{\infty} E_{m} E_{m}^{*} \cdot d x d y}=\frac{\omega^{2} \mu \varepsilon_{p}}{4\left|\beta_{m}\right|} \frac{\iint_{S_{P E R}} E_{m} \cdot E_{m}^{*} \cdot d s}{\int_{-\infty}^{\infty} \int_{-\infty}^{\infty} E_{m} E_{m}^{*} \cdot d x d y}=\frac{\omega^{2} \mu \varepsilon_{p}}{4 \beta_{m}} \cdot \eta= \\
& =\frac{k^{2} 2 n \Delta n_{0}}{4 k n n_{e f f}} \cdot \eta \approx \frac{k \Delta n_{0}}{2} \cdot \eta \\
& \eta=\frac{\iint_{S_{P E R}} E_{m} \cdot E_{m}^{*} \cdot d s}{\int_{-\infty}^{\infty} \int_{-\infty}^{\infty} E_{m} E_{m}^{*} \cdot d x d y} \\
& q=j \frac{k \Delta n_{0}}{2} \cdot \eta
\end{aligned}
$$


where we have assumed a weakly guiding waveguide, i.e. $n_{\text {core }} \approx n_{\text {clad }} \approx n_{\text {eff }}$.

\subsubsection{Simple solutions of coupled equations}

When the coupling coefficient, $q(z)$ is constant it is possible to solve (2-33) analytically. After differentiation of (2-33a) and (2-33b):

$$
\begin{aligned}
& \frac{d^{2} b_{F}}{d z^{2}}=\gamma^{2} b_{F} \\
& \frac{d^{2} b_{B}}{d z^{2}}=\gamma^{2} b_{B}
\end{aligned}
$$

where $\gamma^{2}=|q|^{2}-\delta^{2}$. Solution of $(2-37 \mathrm{a}),(2-37 \mathrm{~b})$ is:

$$
\begin{aligned}
& b_{F}(z)=A_{11} \cdot \exp [\gamma \cdot z]+A_{12} \cdot \exp [-\gamma \cdot z] \\
& b_{B}(z)=A_{21} \cdot \exp [\gamma \cdot z]+A_{22} \cdot \exp [-\gamma \cdot z]
\end{aligned}
$$

Coefficients $A_{11}, A_{12}, A_{21}, A_{22}$ can be found from (2-33a), (2-33b) using boundary conditions of the grating:

$$
b_{F}(z=0)=1 \text { and } b_{B}(z=L)=0,
$$

where $L$ is the length of FBG, i.e. at the beginning of the grating a forward incident mode is launched, normalized to one; whereas at the other end of the grating a backward mode is not present. The values of coefficients are obtained as follows:

$$
\begin{aligned}
& A_{11}=\frac{\gamma+i \delta}{2} \frac{\exp [-\gamma L]}{\cosh (\gamma L)-i \delta \sinh (\gamma L)} \\
& A_{12}=\frac{\gamma-i \delta}{2} \frac{\exp [\gamma L]}{\cosh (\gamma L)-i \delta \sinh (\gamma L)} \\
& A_{21}=\frac{q^{*}}{2} \frac{\exp [-\gamma L]}{\cosh (\gamma L)-i \delta \sinh (\gamma L)} \\
& A_{22}=\frac{-q^{*}}{2} \frac{\exp [\gamma L]}{\cosh (\gamma L)-i \delta \sinh (\gamma L)}
\end{aligned}
$$

Equations (2-38a), (2-38b) can be written in the matrix form: 
$\left[\begin{array}{l}b_{F}(z) \\ b_{B}(z)\end{array}\right]=A \cdot\left[\begin{array}{c}\exp [\gamma \cdot z] \\ \exp [-\gamma \cdot z]\end{array}\right]$

From (2-38a), (2-38b) we can find $b_{F}(z+\Delta), b_{B}(z+\Delta)$ :

$b_{F}(z+\Delta)=A_{11} \cdot \exp [\gamma \cdot \Delta] \cdot \exp [\gamma \cdot z]+A_{12} \cdot \exp [-\gamma \cdot \Delta] \cdot \exp [-\gamma \cdot z]$
$b_{B}(z+\Delta)=A_{21} \cdot \exp [\gamma \cdot \Delta] \cdot \exp [\gamma \cdot z]+A_{22} \cdot \exp [-\gamma \cdot \Delta] \cdot \exp [-\gamma \cdot z]$

or,

$\left[\begin{array}{l}b_{F}(z+\Delta) \\ b_{B}(z+\Delta)\end{array}\right]=\left[\begin{array}{ll}A_{11} \cdot \exp [\gamma \cdot \Delta] & A_{12} \cdot \exp [-\gamma \cdot \Delta] \\ A_{21} \cdot \exp [\gamma \cdot \Delta] & A_{22} \cdot \exp [-\gamma \cdot \Delta]\end{array}\right]\left[\begin{array}{c}\exp [\gamma \cdot z] \\ \exp [-\gamma \cdot z]\end{array}\right]=B \cdot\left[\begin{array}{c}\exp [\gamma \cdot z] \\ \exp [-\gamma \cdot z]\end{array}\right]$ $\ldots(2-43)$

where matrix $B$ is defined as,

$$
B=A \cdot\left[\begin{array}{cc}
\exp [\gamma \cdot \Delta] & 0 \\
0 & \exp [-\gamma \cdot \Delta]
\end{array}\right]
$$

However, it is advantageous to express $b_{F}(z+\Delta), b_{B}(z+\Delta)$ in the basis of functions $b_{F}(z), b_{B}(z)$ through the matrix transformation:

$$
\begin{aligned}
& {\left[\begin{array}{l}
b_{F}(z+\Delta) \\
b_{B}(z+\Delta)
\end{array}\right]=A \cdot\left[\begin{array}{c}
\exp [\gamma(z+\Delta)] \\
\exp [-\gamma(z+\Delta)]
\end{array}\right]=A \cdot\left[\begin{array}{cc}
\exp [\gamma \cdot \Delta] & 0 \\
0 & \exp [-\gamma \cdot \Delta]
\end{array}\right] \cdot\left[\begin{array}{c}
\exp [\gamma \cdot z] \\
\exp [-\gamma \cdot z]
\end{array}\right]=} \\
& =A \cdot\left[\begin{array}{cc}
\exp [\gamma \cdot \Delta] & 0 \\
0 & \exp [-\gamma \cdot \Delta]
\end{array}\right] \cdot A^{-1} \cdot A \cdot\left[\begin{array}{c}
\exp [\gamma \cdot z] \\
\exp [-\gamma \cdot z]
\end{array}\right]= \\
& =A \cdot\left[\begin{array}{cc}
\exp [\gamma \cdot \Delta] & 0 \\
0 & \exp [-\gamma \cdot \Delta]
\end{array}\right] \cdot A^{-1} \cdot\left[\begin{array}{l}
b_{F}(z) \\
b_{B}(z)
\end{array}\right]= \\
& =M \cdot\left[\begin{array}{cc}
b_{F}(z) \\
b_{B}(z)
\end{array}\right] \\
& M=A \cdot\left[\begin{array}{cc}
\exp [\gamma \cdot \Delta] & 0 \\
0 & \exp [-\gamma \cdot \Delta]
\end{array}\right] \cdot A^{-1}
\end{aligned}
$$

$M$ is called the transfer matrix, its elements are:

$$
M_{11}=\cosh (\gamma \cdot \Delta)+i \frac{\delta}{\gamma} \sinh (\gamma \cdot \Delta)
$$


$M_{12}=i \frac{q}{\gamma} \sinh (\gamma \cdot \Delta)$

$M_{21}=i \frac{q^{*}}{\gamma} \sinh (\gamma \cdot \Delta)$

$M_{22}=\cosh (\gamma \cdot \Delta)-i \frac{\delta}{\gamma} \sinh (\gamma \cdot \Delta)$

\subsubsection{Transfer matrix for FBG}

$M=\left[\begin{array}{cc}\cosh (\gamma \cdot \Delta)+i \frac{\delta}{\gamma} \sinh (\gamma \cdot \Delta) & i \frac{q^{*}}{\gamma} \sinh (\gamma \cdot \Delta) \\ i \frac{q}{\gamma} \sinh (\gamma \cdot \Delta) & \cosh (\gamma \cdot \Delta)-i \frac{\delta}{\gamma} \sinh (\gamma \cdot \Delta)\end{array}\right]$

With the help of transfer matrix elements we can find the amplitude reflection coefficient of uniform grating:

$r(\delta)=\frac{b_{B}(z=0, \delta)}{b_{F}(z=0, \delta)}=-\frac{M_{21}}{M_{22}}$
$r(\delta)=-\frac{i \frac{q}{\gamma} \sinh (\gamma \cdot L)}{\cosh (\gamma \cdot L)-i \frac{\delta}{\gamma} \sinh (\gamma \cdot L)}$

again, with boundary conditions:

$b_{F}(z=0, \delta)=1, b_{B}(z=L, \delta)=0$

In Fig. 2-1 a few graphs are illustrating the spectral reflectivities for different values of the product $q L$ when the grating is uniform.

In many cases it is useful to know the inverse of the transfer matrix, i.e. matrix that relates $b_{F}(z), b_{B}(z)$ through $b_{F}(z+\Delta), b_{B}(z+\Delta)$ : 
$\left[\begin{array}{l}b_{F}(z+\Delta) \\ b_{B}(z+\Delta)\end{array}\right]=M \cdot\left[\begin{array}{l}b_{F}(z) \\ b_{B}(z)\end{array}\right]$

$\left[\begin{array}{l}b_{F}(z) \\ b_{B}(z)\end{array}\right]=M^{-1} \cdot\left[\begin{array}{l}b_{F}(z+\Delta) \\ b_{B}(z+\Delta)\end{array}\right]$

The coefficients of $M^{-1}$ are easy to find:

$$
M^{-1}=\left[\begin{array}{cc}
\cosh (\gamma \cdot \Delta)-i \frac{\delta}{\gamma} \sinh (\gamma \cdot \Delta) & -i \frac{q^{*}}{\gamma} \sinh (\gamma \cdot \Delta) \\
-i \frac{q}{\gamma} \sinh (\gamma \cdot \Delta) & \cosh (\gamma \cdot \Delta)+i \frac{\delta}{\gamma} \sinh (\gamma \cdot \Delta)
\end{array}\right]
$$

The matrix formulation is useful in calculating the response of non-uniform gratings as a concatenation of uniform segments.

$\left[\begin{array}{l}b_{F}\left(z_{N}\right) \\ b_{B}\left(z_{N}\right)\end{array}\right]=M_{N} \cdot M_{N-1} \cdot \ldots \cdot M_{2} \cdot M_{1} \cdot\left[\begin{array}{l}b_{F}\left(z_{1}\right) \\ b_{B}\left(z_{1}\right)\end{array}\right]$

Then the transfer matrix of the whole grating is equal to the product of transfer matrices of each section of the grating. That is shown in Fig. 2-2. 


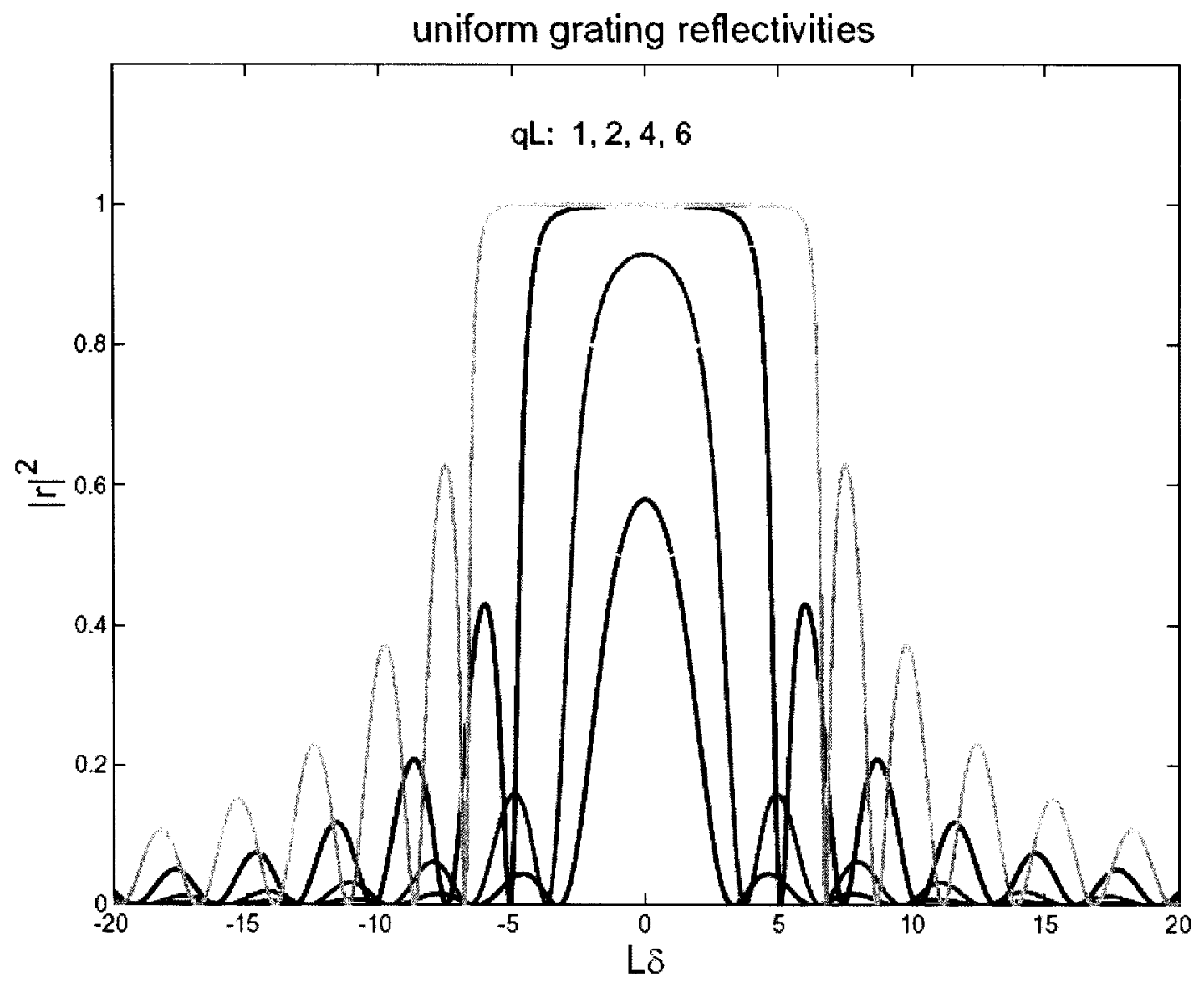

Fig. 2-1. FBG reflectivity with constant coupling coefficient. 


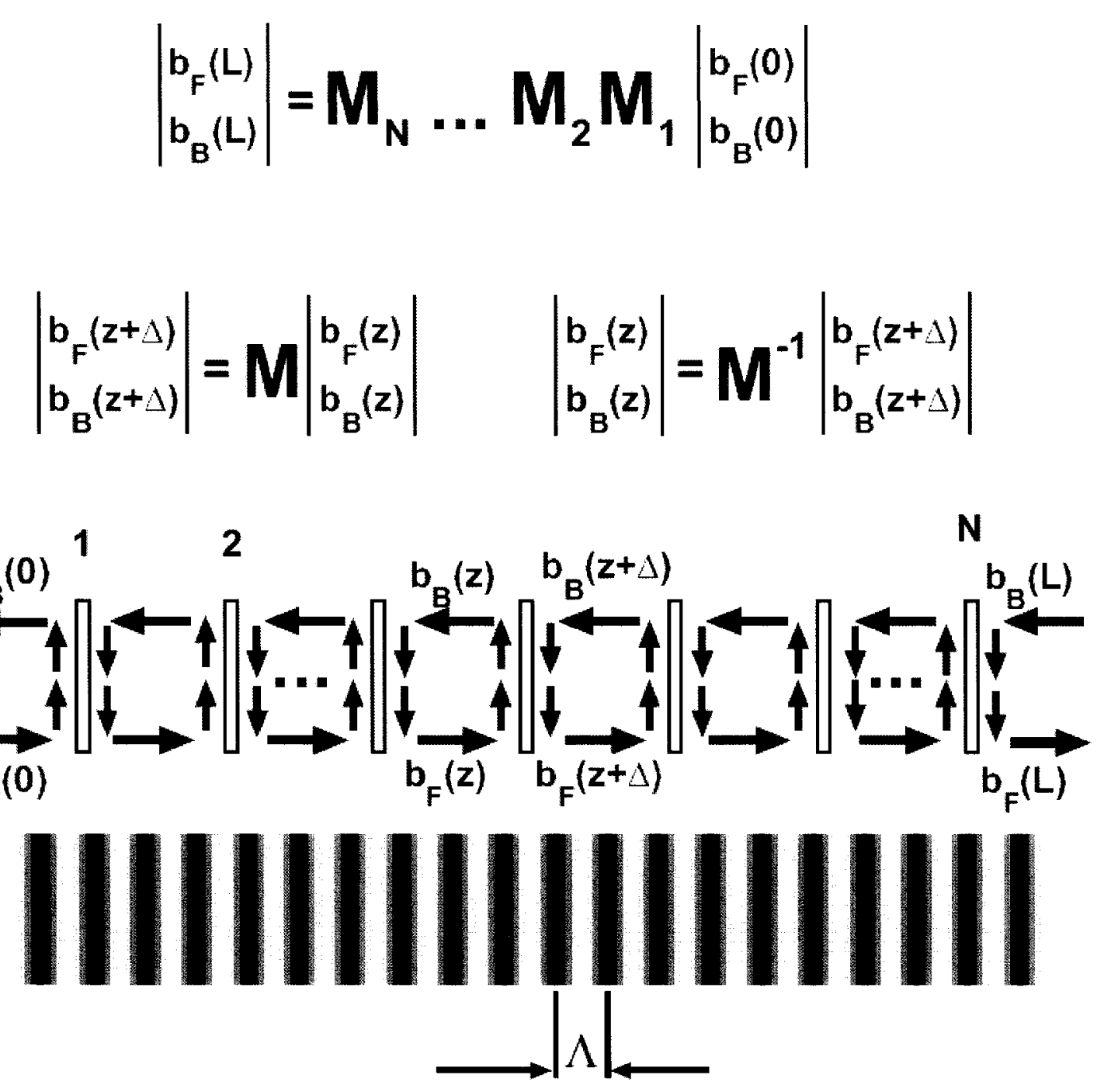

Fig. 2-2. $\quad M$ is a transfer matrix for uniform grating. 


\subsubsection{Analytical solution for non-uniform gratings}

The accuracy of this matrix multiplication method is limited by the required discretization of the grating into a set of uniform gratings. Furthermore, it requires numerical calculations. When the grating coupling is weak, an approximate simple relation between coupling and reflection can also be found analytically.

Define the local reflectivity, $r(z, \delta)$ as:

$$
r(z, \delta)=\frac{b_{B}(z, \delta)}{b_{F}(z, \delta)}
$$

and define:

$$
R(z, \delta)=r(z, \delta) \cdot \exp [i \cdot 2 \delta z]
$$

We can physically measure the reflectivity of FBG only at $z=0$

$$
R(0, \delta)=r(0, \delta)=r(\delta)
$$

After taking the derivative of (2-55):

$$
\frac{d R}{d z}=\left(\frac{1}{b_{B}} \frac{d b_{B}}{d z}-\frac{1}{b_{F}} \frac{d b_{F}}{d z}+i \cdot 2 \delta\right) \frac{b_{B}}{b_{F}} \exp [i \cdot 2 \delta z]
$$

Combining the last result with (2-56), (2-33a) and (2-33b) we get:

$$
\frac{d R}{d z}=q^{*} \exp [i \cdot 2 \delta z]-q R^{2} \exp [-i \cdot 2 \delta z]
$$

This is known as the Ricatti equation. Neglecting the second term, since $R^{2} \ll<$, the equation becomes:

$$
\frac{d R}{d z} \approx q^{*} \exp [i \cdot 2 \delta z]
$$

This can be integrated analytically:

$$
R(L, \delta)-R(0, \delta) \approx \int_{0}^{L} q^{*} \exp [i \cdot 2 \delta z] d z
$$

From the boundary conditions, $b_{F}(0, \delta)=1$ and $b_{B}(L, \delta)=0,($ hence $R(L, \delta)=0)$. 
Finally, the spectral response at the beginning of the FBG can be related to grating coupling as:

$$
r(\delta) \approx-\int_{0}^{L} q^{*}(z) \exp [i \cdot 2 \delta z] d z
$$

We see that in this regime the spectral response is given by the Fourier transform of the coupling coefficient of the grating.

This also helps in solving the inverse problem of finding the required coupling coefficient to achieve a given reflectivity profile distribution along $z$. The inverse problem can be solved relatively easy, just by taking the inverse Fourier transform from the spectral response.

When $q^{*}(z)$ is constant, the reflectivity from (2-62) is:

$r(\delta) \approx-q^{*} \frac{\sin (\delta L)}{\delta} \exp (i \delta L)$

In Fig. 2-3 are a few graphs illustrating this relation for different values of the product $q L$. Limitations of this approach: since the Fourier transform is a linear transformation, if a given function is multiplied by a constant factor, the Fourier image-function is multiplied by the same factor. From that observation we see that simple expression (2-62) can not be used in a general case: the spectral response $r(\delta)$ can not exceed 1 by definition, however the $(q L)$ coupling coefficient of the grating has no limit in general. This implies that as $q(z)$ gets higher, the simple relation (2-62) is no longer valid.

In cases of strong coupling, the design problem of finding a proper coupling coefficient $q(z)$ for a given spectral response $r(\delta)$ has to be solved numerically. 


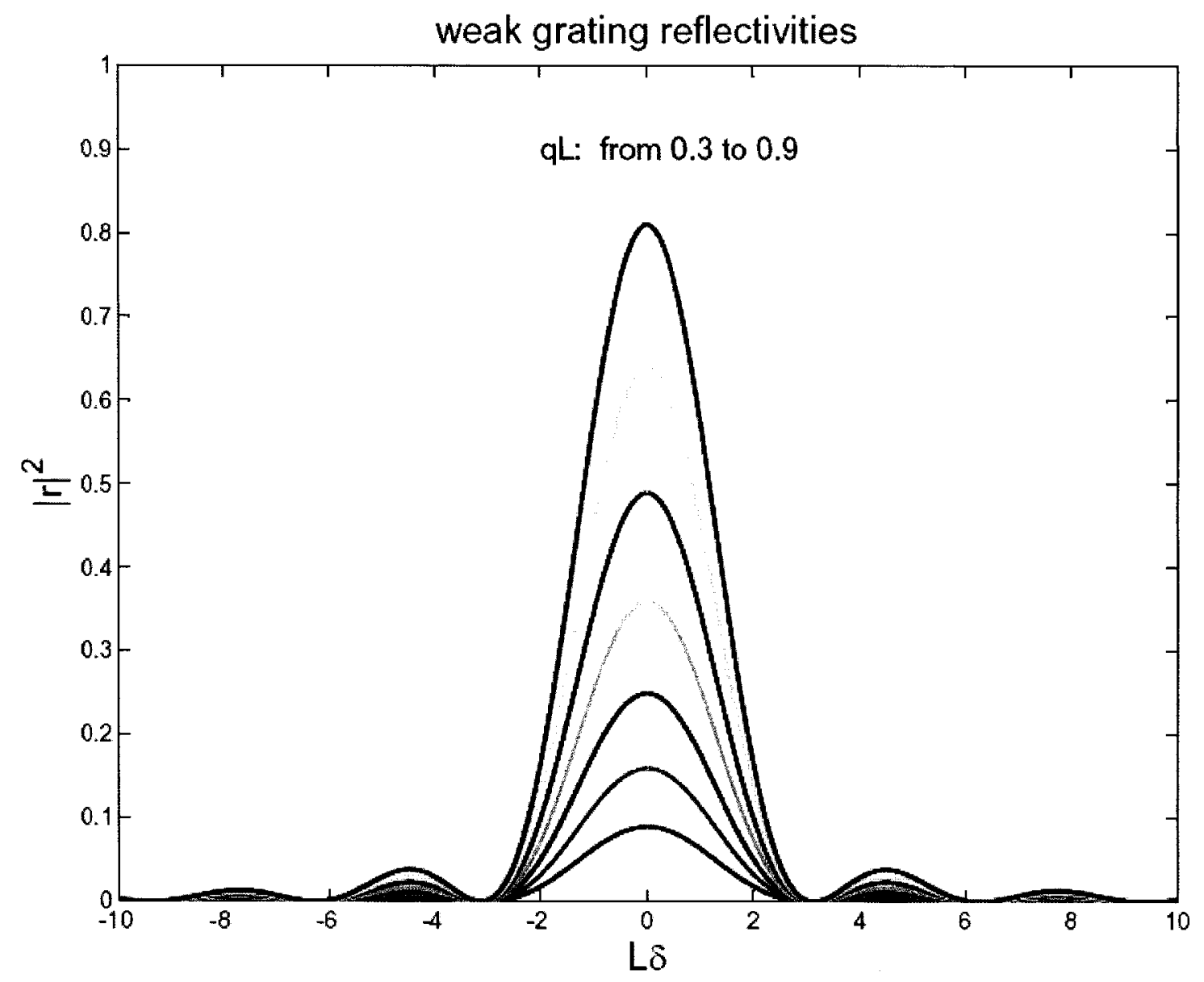

Fig. 2-3. $\quad$ FBG reflectivity with weak coupling coefficient.

\subsection{Codirectional coupling (CMT for LPG)}

Codirectional coupling between two modes, propagating forward, is described by equations (2-26).

$$
\begin{aligned}
& \frac{d A}{d z}=-j B \kappa \exp [j 2 \delta z] \\
& \frac{d A_{l}}{d z}=-j A_{m} \kappa^{*} \exp [-j 2 \delta z]
\end{aligned}
$$


where $\kappa=D_{i m}$,

$\delta=\frac{1}{2}\left(\beta_{m}-\beta_{l}\right)-\frac{\pi}{\Lambda}$.

With the substitution,

$R=A_{m} \exp [-j \delta z]$,

$S=A_{l} \exp [j \delta z]$

the above equations become,

$\frac{d R}{d z}=-j \delta R-j \kappa S$

$\frac{d S}{d z}=j \delta S-j \kappa^{*} R$

They can be solved in a similar fashion as the equations for codirectional coupling. We present the result for the transfer matrix below, ([31] p. 1286):

$M_{C O}=\left[\begin{array}{cc}\cos (\gamma \cdot \Delta)+i \frac{\delta}{\gamma} \sin (\gamma \cdot \Delta) & i \frac{\kappa^{*}}{\gamma} \sin (\gamma \cdot \Delta) \\ i \frac{\kappa}{\gamma} \sinh (\gamma \cdot \Delta) & \cos (\gamma \cdot \Delta)-i \frac{\delta}{\gamma} \sin (\gamma \cdot \Delta)\end{array}\right]$

where $\gamma^{2}=\kappa^{2}+\delta^{2}$.

Usually this type of coupling is used to couple a core mode to a cladding mode. Over a distance $\Delta$ we get:

$\left|\begin{array}{l}E_{C L A D}(z+\Delta) \\ E_{C O R E}(z+\Delta)\end{array}\right|=M_{C O} \cdot\left|\begin{array}{l}E_{C L A D}(z) \\ E_{C O R E}(z)\end{array}\right|$

and if $E_{C L A D}(z)=0$ and $E_{C O R E}(z)=1$,

then the transmission characteristic for the electric field is given by:

$T_{E}=\frac{E_{\text {CORE }}(z+\Delta)}{E_{\text {CORE }}(z)}=M_{22}$

The power transmission characteristic of the codirectional coupler and therefore LPG is: 


$$
\begin{aligned}
& T_{P}=\left|M_{22}\right|^{2}=\cos ^{2}(\gamma \cdot \Delta)+\frac{\delta^{2}}{\gamma^{2}} \sin ^{2}(\gamma \cdot \Delta)=1-\sin ^{2}(\gamma \cdot \Delta)+\frac{\delta^{2}}{\gamma^{2}} \sin ^{2}(\gamma \cdot \Delta)= \\
& =1-\left(1-\frac{\delta^{2}}{\gamma^{2}}\right) \sin ^{2}(\gamma \cdot \Delta)=1-\frac{\kappa^{2}}{\gamma^{2}} \sin ^{2}(\gamma \cdot \Delta)=1-\frac{\sin ^{2}\left(\kappa \cdot \Delta \cdot \sqrt{1+\frac{\delta^{2}}{\kappa^{2}}}\right)}{1+\frac{\delta^{2}}{\kappa^{2}}} \\
& T_{P_{-} L P G}=1-\frac{\sin ^{2}\left(\kappa \cdot \Delta \cdot \sqrt{1+\frac{\delta^{2}}{\kappa^{2}}}\right)}{1+\frac{\delta^{2}}{\kappa^{2}}}
\end{aligned}
$$




\section{Chapter 3}

\section{Five-layer slab theory}

The goal of this chapter is to provide modal solutions (fields and effective indices) for the waveguiding structures considered in this work. Planar dielectric waveguides are treated in many standard textbooks, see for example [32], [33].

In section 3.1 we start with Maxwell's equation for dielectric media and derive the general form of the Helmholtz wave equation for transverse electric (TE) mode, which is valid for a slab waveguide with multiple layers. In section 3.2 the wave equation is applied specifically to a three-layer symmetric waveguide problem and the propagation constant is derived in Appendix D. The solution for the three-layer symmetric waveguide is a starting point for our next problem: the five-layer waveguide, considered in section 3.3. We treat the five-layer waveguide as a three-layer waveguide with an additional second guided channel. That second channel is built up gradually, first its refractive index is considered to be equal to the background (i.e. there is no channel yet), then its refractive index starts to 'slowly' increase forming the actual channel. Such an approach, 'slowly' building up a second channel on a three-layer slab, allows us to use perturbation theory and finally get a solution for the fivelayer slab based on a known solution for the three-layer slab.

Why are we concerned with a five-layer slab, and why is this geometry structure important? From the literature survey (see chapter 1) we know that fiber with an additional layer deposited on the cladding possesses very high sensitivity to external refractive index variations. Such a fiber is similar to a structure with two channels (core and deposited layer) separated by a cladding, it is equivalent to a five-layer slab waveguide. We are looking for a structure that will be sensitive to an internal refractive index variation and therefore suitable for a nonlinear optical switching application. 


\subsection{Wave equation for dielectric planar waveguide}

Maxwell's equations are treated in numerous textbooks, see for example [32], [33]. The general form of Maxwell's equations for any media is given by,

$\nabla \times \vec{E}=-\frac{\partial \vec{B}}{\partial t}$

$\nabla \times \vec{H}=\frac{\partial \vec{D}}{\partial t}+\vec{J}$

$\nabla \cdot \vec{D}=\rho$

$\nabla \cdot \vec{B}=0$

where,

$\vec{E}$ is electric field amplitude in $\left[\mathrm{V} \cdot \mathrm{m}^{-1}\right]$, volts per meter.

$\vec{H}$ is magnetic field amplitude in $\left[\mathrm{A} \cdot \mathrm{m}^{-1}\right]$, amps per meter.

$\vec{D}$ is electric flux density in $\left[\mathrm{C} \cdot \mathrm{m}^{-2}\right]$, Coulombs per meter square.

$\vec{B}$ is magnetic flux density in $\left[\mathrm{Wb} \cdot \mathrm{m}^{-2}\right]$, Webers per meter square.

$\vec{D}$ is electric flux density in $\left[\mathrm{C} \cdot \mathrm{m}^{-2}\right]$, Coulombs per meter square.

$\vec{J}$ is current density in $\left[\mathrm{A} \cdot \mathrm{m}^{-2}\right]$, amps per meter square.

$\rho$ is free charge density in $\left[\mathrm{C} \cdot \mathrm{m}^{-3}\right]$, Coulombs per meter cube.

Applying equations (3-1), (3-2), (3-3), (3-4) to dielectric media which do not have free charges and currents, $\rho=0, \vec{J}=0$ and assuming time dependence as $\exp [j \omega t]$ for $\vec{B}, \vec{D}$ we get:

$\nabla \times \vec{E}=-j \omega \vec{B}$

$\nabla \times \vec{H}=j \omega \vec{D}$

$\nabla \cdot \vec{D}=\rho$

$\nabla \cdot \vec{B}=0$

In addition we have constitutive relations between $\vec{D}$ and $\vec{E}$, and between $\vec{B}$ and $\vec{H}$, 
$\vec{D}=\varepsilon \vec{E}$

$\vec{B}=\mu \vec{H}$

where

$\varepsilon=\varepsilon_{0} \varepsilon_{r}$

$\varepsilon_{0}$ permittivity of free space in $\left[\frac{\mathrm{F}}{\mathrm{m}}\right]$, Farads per meter. $\varepsilon_{0}=8.854 \cdot 10^{-12}\left[\frac{\mathrm{F}}{\mathrm{m}}\right]$

$\varepsilon_{r}$ relative permittivity of the media, dimensionless.

$\mu=\mu_{0} \mu_{r}$

$\mu_{0}$ permeability of free space in $\left[\frac{\mathrm{H}}{\mathrm{m}}\right]$, Henrys per meter. $\mu_{0}=4 \pi \cdot 10^{-7}\left[\frac{\mathrm{H}}{\mathrm{m}}\right]$

$\mu_{r}$ permeability of the media, dimensionless.

Eliminating $\vec{D}$ and $\vec{B}$ from (3-1a), (3-2a):

$\nabla \times \vec{E}=-j \omega \mu \vec{H}$

$\nabla \times \vec{H}=j \omega \varepsilon \vec{E}$

$\nabla \times \vec{E}=\left|\begin{array}{ccc}\hat{x} & \hat{y} & \hat{z} \\ \frac{\partial}{\partial x} & \frac{\partial}{\partial y} & \frac{\partial}{\partial z} \\ E_{x} & E_{y} & E_{z}\end{array}\right|=\hat{x}\left(\frac{\partial}{\partial y} E_{z}-\frac{\partial}{\partial z} E_{y}\right)+\hat{y}\left(\frac{\partial}{\partial z} E_{x}-\frac{\partial}{\partial x} E_{z}\right)+\hat{z}\left(\frac{\partial}{\partial x} E_{y}-\frac{\partial}{\partial y} E_{x}\right)$

$-j \omega \mu H_{x}=\frac{\partial}{\partial y} E_{z}-\frac{\partial}{\partial z} E_{y}$

$-j \omega \mu H_{y}=\frac{\partial}{\partial z} E_{x}-\frac{\partial}{\partial x} E_{z}$

$-j \omega \mu H_{z}=\frac{\partial}{\partial x} E_{y}-\frac{\partial}{\partial y} E_{x}$ 
$\nabla \times \vec{H}=\left|\begin{array}{ccc}\hat{x} & \hat{y} & \hat{z} \\ \frac{\partial}{\partial x} & \frac{\partial}{\partial y} & \frac{\partial}{\partial z} \\ H_{x} & H_{y} & H_{z}\end{array}\right|=\hat{x}\left(\frac{\partial}{\partial y} H_{z}-\frac{\partial}{\partial z} H_{y}\right)+\hat{y}\left(\frac{\partial}{\partial z} H_{x}-\frac{\partial}{\partial x} H_{z}\right)+\hat{z}\left(\frac{\partial}{\partial x} H_{y}-\frac{\partial}{\partial y} H_{x}\right)$

$j \omega \varepsilon E_{x}=\frac{\partial}{\partial y} H_{z}-\frac{\partial}{\partial z} H_{y}$

$j \omega \varepsilon E_{y}=\frac{\partial}{\partial z} H_{x}-\frac{\partial}{\partial x} H_{z}$

$j \omega \varepsilon E_{z}=\frac{\partial}{\partial x} H_{y}-\frac{\partial}{\partial y} H_{x}$

Assuming the dielectric media is homogeneous along z-axis, then all field components have $\exp (-j \beta z)$ dependence along $z$-axis and operation $\frac{\partial}{\partial z}$ is equivalent to $(-j \beta), \frac{\partial}{\partial z} \Leftrightarrow-j \beta$

$-j \omega \mu H_{x}=\frac{\partial}{\partial y} E_{z}+j \beta E_{y}$

$-j \omega \mu H_{y}=-j \beta E_{x}-\frac{\partial}{\partial x} E_{z}$

$-j \omega \mu H_{z}=\frac{\partial}{\partial x} E_{y}-\frac{\partial}{\partial y} E_{x}$

$j \omega \varepsilon E_{x}=\frac{\partial}{\partial y} H_{z}+j \beta H_{y}$

$j \omega \varepsilon E_{y}=-j \beta H_{x}-\frac{\partial}{\partial x} H_{z}$

$j \omega \varepsilon E_{z}=\frac{\partial}{\partial x} H_{y}-\frac{\partial}{\partial y} H_{x}$

Applying the curl operation to equation $(3-1 b): \nabla \times(3-1 b)$

$\nabla \times(\nabla \times \vec{E})=-j \omega \mu \nabla \times \vec{H}$

Using the vector identity: $\nabla \times(\nabla \times \vec{E})=\nabla \cdot(\nabla \cdot \vec{E})-\nabla^{2} \vec{E}$ and (3-3a),(3-5): 
$-\nabla^{2} \vec{E}=-j \omega \mu \nabla \times \vec{H}$

Using (3-2b):

$\nabla^{2} \vec{E}+\omega^{2} \mu \varepsilon \vec{E}=0$

The above equation is called Helmholtz wave equation.

Similarly, by applying the curl operator to equation (3-2b) we get wave equation for magnetic field $\vec{H}$ :

$\nabla^{2} \vec{H}+\omega^{2} \mu \varepsilon \vec{H}=0$

Our media is a planar waveguide that is homogeneous along the y-axis, so any quantity

considered will be constant along y-axis, therefore $\frac{\partial}{\partial y}=0$. Assuming, we are dealing only

with TE mode, i.e. $E_{z}=0$. Then,

$-j \omega \mu H_{x}=j \beta E_{y}$

$-j \omega \mu H_{y}=-j \beta E_{x}$

$-j \omega \mu H_{z}=\frac{\partial}{\partial x} E_{y}$

$j \omega \varepsilon E_{x}=j \beta H_{y}$

$j \omega \varepsilon E_{y}=-j \beta H_{x}-\frac{\partial}{\partial x} H_{z}$

$j \omega \varepsilon E_{z}=\frac{\partial}{\partial x} H_{y}=0$

$\Rightarrow H_{y}=\operatorname{const}(x) \Rightarrow H_{y}=0$ this follows from physical considerations, $H_{y}$ cannot be a nonzero constant at $x=+\infty$, therefore that constant must be zero. Then,

$-j \omega \mu H_{x}=j \beta E_{y}$

$H_{y}=0$

$-j \omega \mu H_{z}=\frac{\partial}{\partial x} E_{y}$

$E_{x}=0$ 
$j \omega \varepsilon E_{y}=-j \beta H_{x}-\frac{\partial}{\partial x} H_{z}$

$E_{z}=0$

\subsection{Three-layer symmetric slab waveguide. [32], [33]}

Consider a three-layer symmetric slab waveguide shown in Fig. 3-1. Tangential fields components (tangential to the interfaces layer-to-layer, each layer is parallel to the plane $x=$ const as in Fig. 3-1) are: $E_{y} \neq 0, E_{z}=0, H_{y}=0, H_{z} \neq 0$.

The wave equation for $E_{y}$ in a slab guide:

$$
\begin{aligned}
& \left(\frac{\partial^{2}}{\partial x^{2}}+\frac{\partial^{2}}{\partial z^{2}}\right) E_{y}+\omega^{2} \mu \varepsilon E_{y}=0 \\
& E_{y}(x, y, z)=e_{y}(x) \cdot \exp [-j \beta z] \\
& H_{z}(x, y, z)=h_{z}(x) \cdot \exp [-j \beta z] \\
& \omega^{2} \mu \varepsilon=\frac{\omega^{2}}{v^{2}}=\frac{\omega^{2} n^{2}}{c^{2}}=k^{2} n^{2}=\left(\frac{2 \pi}{\lambda}\right)^{2} n^{2} \\
& \frac{d^{2}}{d x^{2}} e_{y}+\left(k^{2} n^{2}-\beta^{2}\right) e_{y}=0
\end{aligned}
$$

The solution of this equation for the eigenmode $e_{y}$ and corresponding eigenvalue $\beta$ depends on relation $k n$.

$$
\begin{aligned}
& h_{z}=\frac{j}{\omega \mu} e_{y} \\
& k^{2} n^{2}(x)=\left\{\begin{array}{cc}
k^{2} n_{b}^{2} & x<0 \\
k^{2} n_{c}^{2} & 0 \leq x \leq \mathrm{v} \\
k^{2} n_{b}^{2} & \mathrm{x}>\mathrm{v}
\end{array}\right.
\end{aligned}
$$




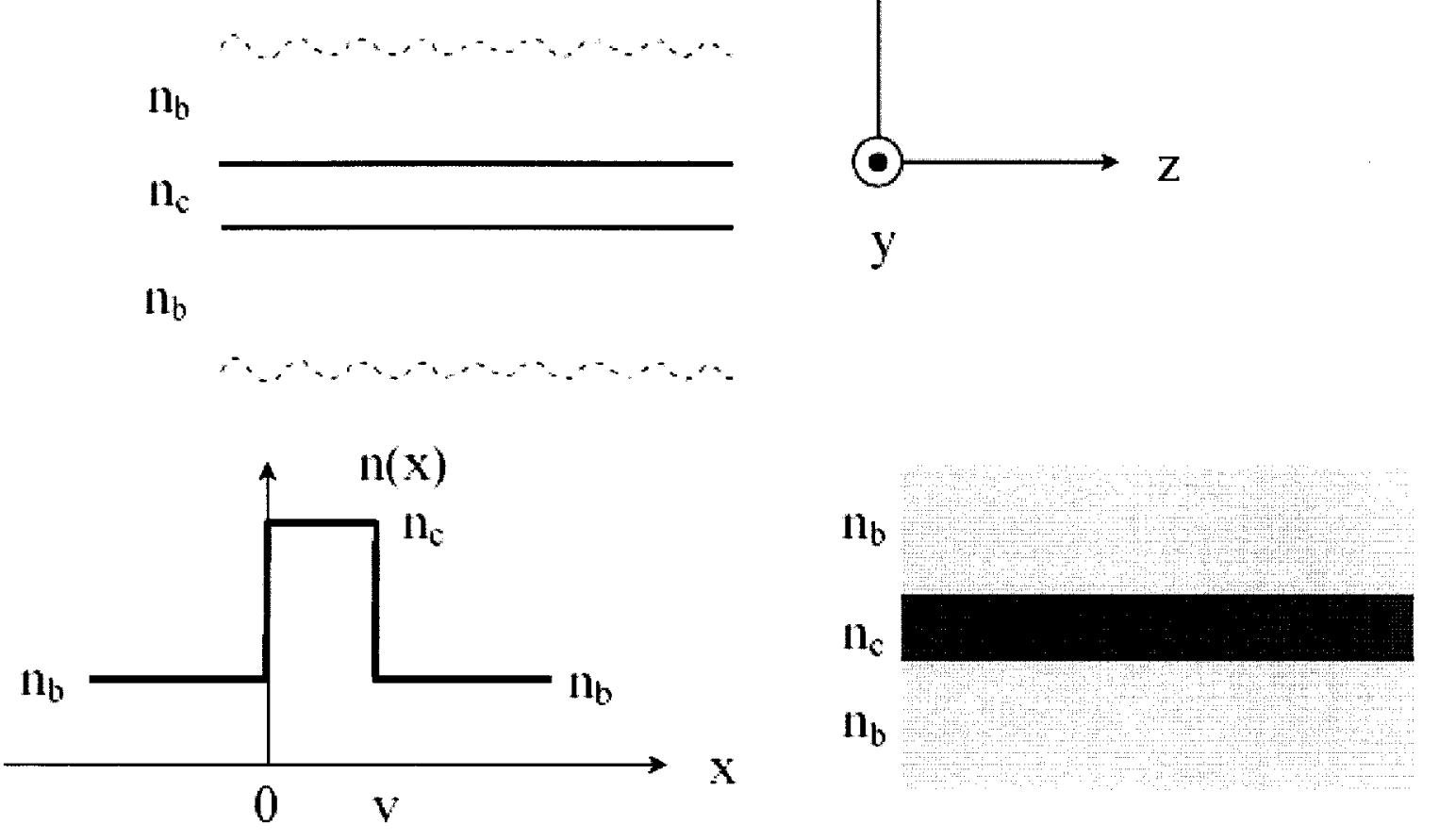

Fig. 3-1. Dielectric slab geometry.

The boundary condition requires that tangential field components be continuous across each interface, i.e. $E_{y}$ and $H_{z}$ must be continuous: 
$e_{y}\left(x=0^{-}\right)=e_{y}\left(x=0^{+}\right)$

$\frac{d}{d x} e_{y}\left(x=0^{-}\right)=\frac{d}{d x} e_{y}\left(x=0^{+}\right)$

$e_{y}\left(x=\mathrm{v}^{-}\right)=e_{y}\left(x=\mathrm{v}^{+}\right)$

$\frac{d}{d x} e_{y}\left(x=\mathrm{v}^{-}\right)=\frac{d}{d x} e_{y}\left(x=\mathrm{v}^{+}\right)$

The scalar Helmholtz wave equation (3-22) with refractive index profile given by (3-24) and boundary conditions for the TE mode $(3-25 a, b)$ is equivalent to the stationary onedimensional Schrödinger equation

$\frac{d^{2}}{d x^{2}} \psi(x, E)-\frac{2 m}{\hbar^{2}}(U(x)-E) \cdot \psi(x, E)=0$

for a particle of mass $m$ in potential energy profile $U(x)$, with $E$ being the energy levels corresponding to eigenfunctions $\psi(x, E)$.

The modal propagation constant corresponds to the energy eigenvalue, $\beta^{2} \leftrightarrow \frac{2 m}{\hbar^{2}} E$, while the refractive index profile corresponds to the potential energy profile, $k^{2} n^{2}(x) \leftrightarrow \frac{2 m}{\hbar^{2}} U(x)$. The difference is the reversed sign in the second term of the equations. The solution of the Helmholtz wave equation for the slab $\left(e_{y}(x, \beta)\right)$ is identical to the wavefunction of the particle in square potential well (see Fig. 3-2).

This analogy suggests that we can use tools developed in quantum mechanics to solve some optical waveguide problems. One of these tools is perturbation theory for stationary states (see Appendix B for details). We apply a perturbative approach to the three-layer slab in order to find the propagation constant for a five-layer slab, assuming that the propagation constant for a three-layer slab is already known (the solution of the propagation constant for a three-layer slab is derived in Appendix D). Fig. 3-3a, b illustrate this approach. We start with the index profile of the three-layer slab then apply a small index perturbation to the position of the second channel and use perturbation theory to evaluate propagation constant for that index profile. Then we can in principle apply a perturbation approach again for a 
slightly larger increment in the second channel. After a couple of steps the refractive index in the second channel can reach an appropriate value.

The dispersion equation for the three-layer slab is:

$\left(u_{c}^{2}-u_{b}^{2}\right) \sin \left(u_{c} \mathrm{v}\right)-2 u_{c} u_{b} \cos \left(u_{c} \mathrm{v}\right)=0$

(see Appendix C for details) 

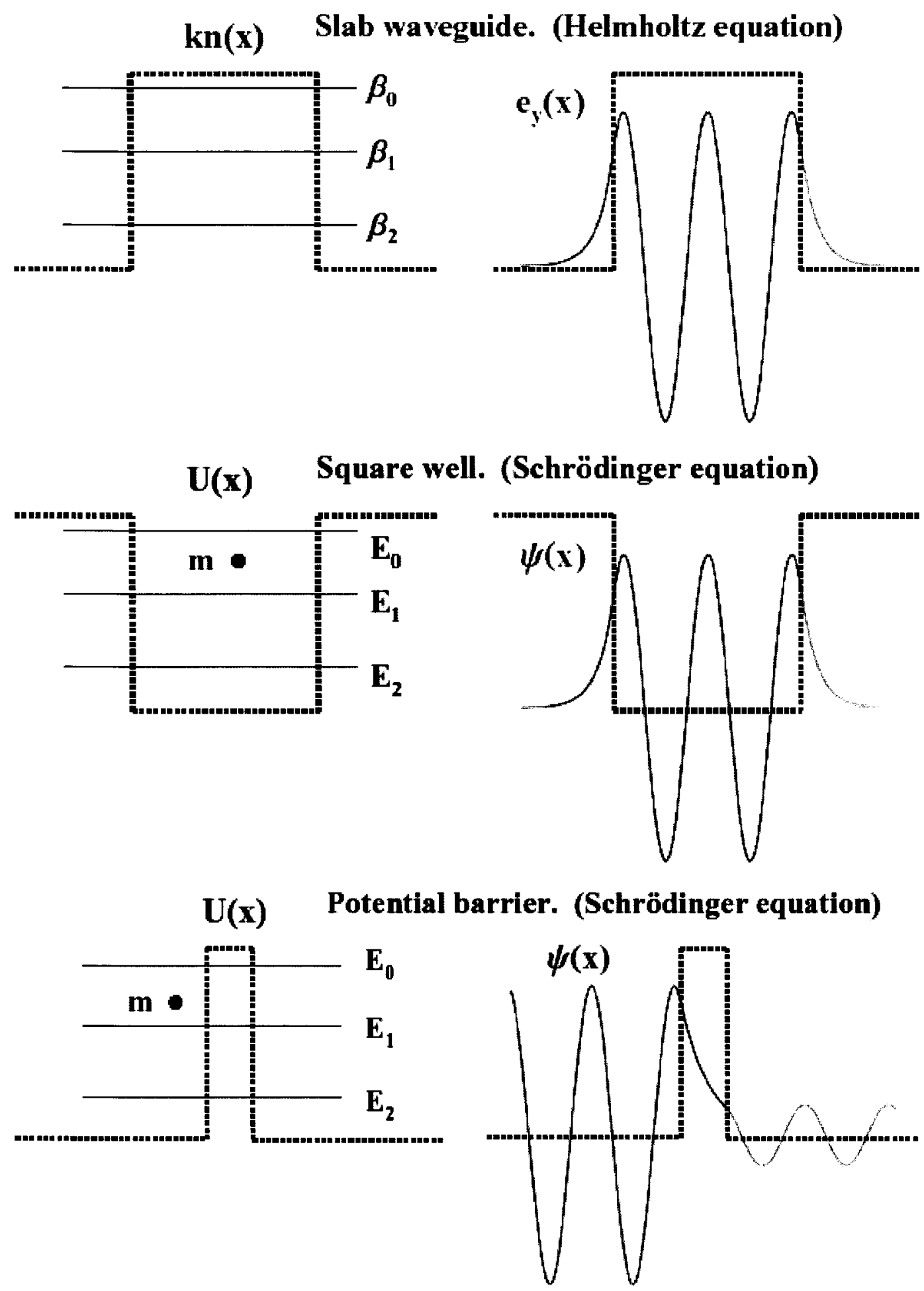

Fig. 3-2. Analogy between slab waveguide problem (Helmholtz equation) and square well potential problem (Schrödinger equation). 

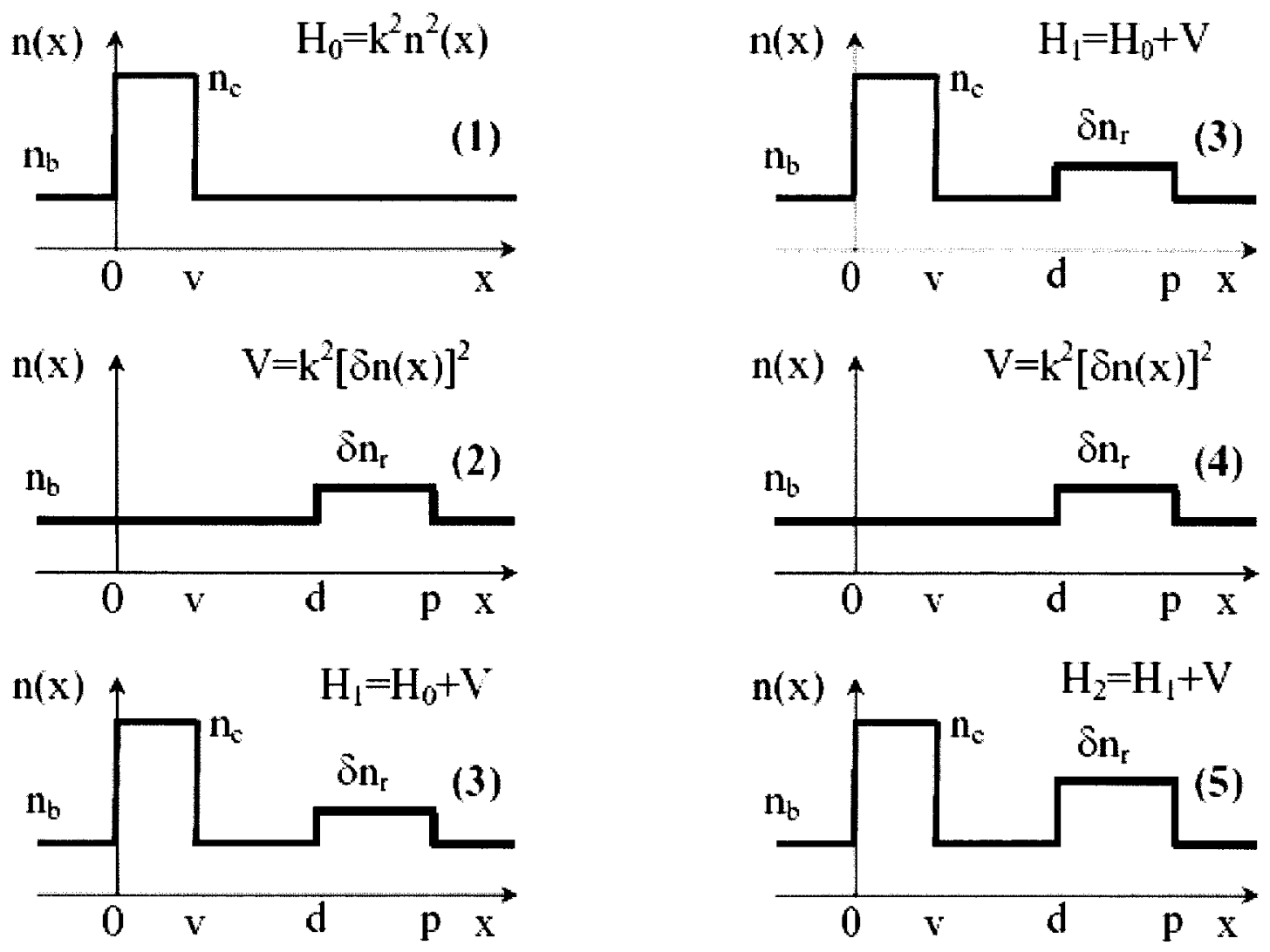

Fig. 3-3a. Successive perturbation approach to the 3-layer slab waveguide generates a slab directional coupler (five-layer slab).

(1) Hamiltonian for 3-layer slab.

(2) small perturbation potential at a location of second channel.

(3) total Hamiltonian combined corresponds to 5-layer slab profile with small refractive index in the second channel.

(4), (5) second step of increment results in larger refractive index in the second channel. 


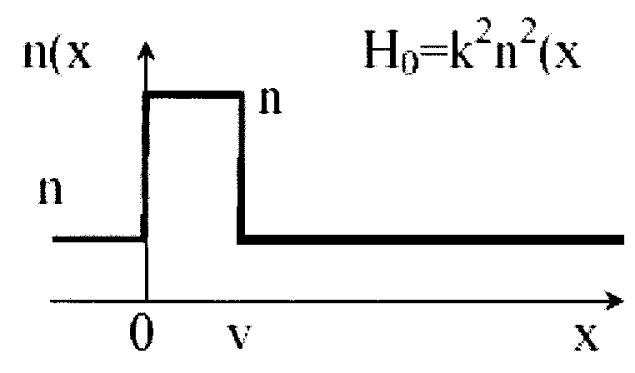

$$
\mathrm{H}_{\mathrm{n}}=\mathrm{H}_{0}+\mathrm{n}
$$
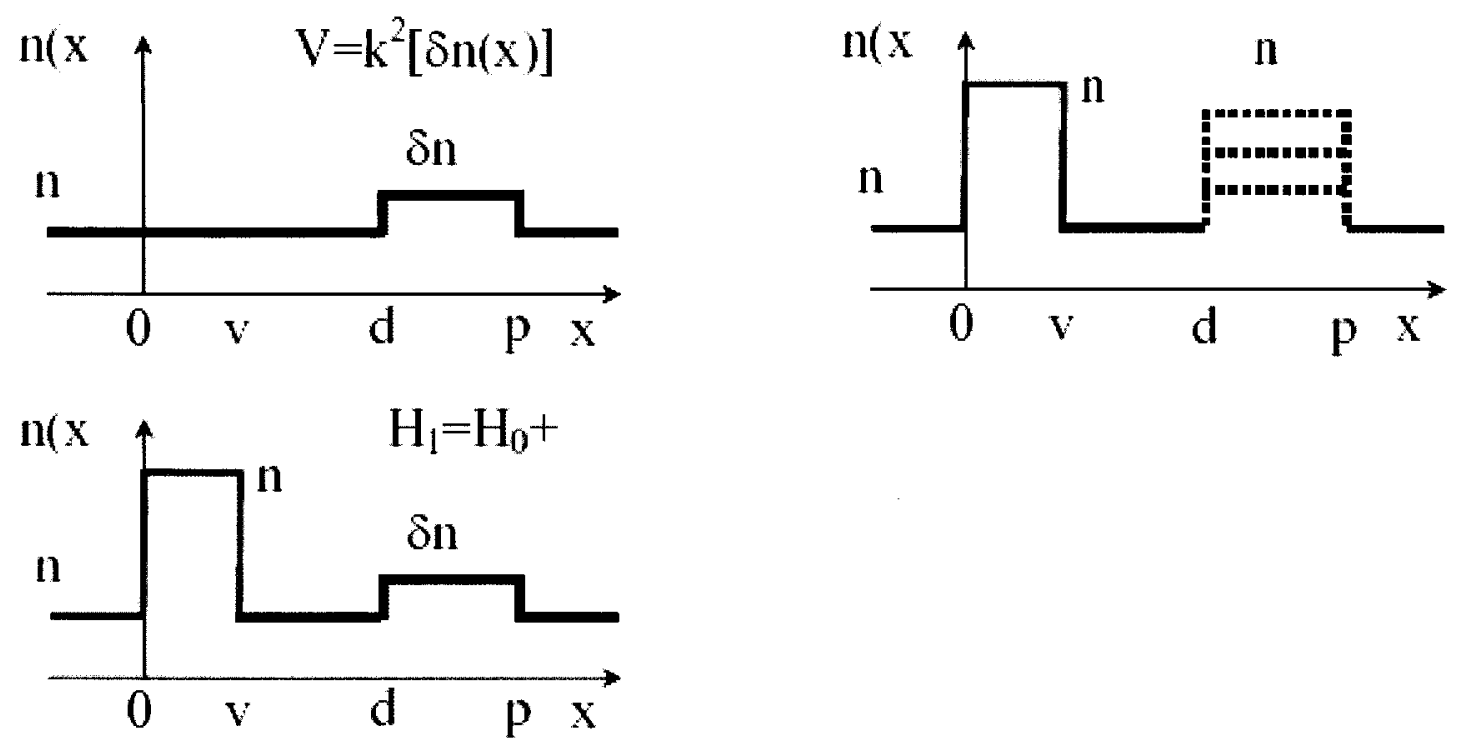

Fig. 3-3b. By repeating perturbative increment many times, we can build up appropriate value of refractive index in the second channel.

\subsection{Five-layer slab waveguide}

The five-layer W-type of symmetric slab is treated in detail in reference [33]. More general, but still symmetric, structures are presented in [35] for both TE and TM modes. We will follow somewhat closely references [36] and [37] where multilayer nonsymmetrical slab 
waveguides were studied, and their field distribution and an explicit eigenvalue equation for the propagation constant were obtained.

In this thesis, we will develop a formalism which allows us to find mode profile and propagation constants as a function of the five-layer slab parameters. In particular, a methodology for obtaining effective mode indices as a function of the refractive index of the five-layer slab will be given.

The electric field in a waveguide with refractive index $n$ can be represented by a superposition of plane waves of the form $E_{0} \exp [j(\vec{k} \bullet \vec{r})]$.

$$
\begin{aligned}
& E_{0} \exp [j(\vec{k} \cdot \vec{r})]=E_{0} \exp \left[j\left(k_{x} x+\beta z\right)\right]=E_{0} \exp \left[j\left(k_{x} x\right)\right] \exp [j(\beta z)]= \\
& =E_{0} \exp \left[j\left(\sqrt{(n k)^{2}-\beta^{2}}\right) x\right] \exp [j(\beta z)]
\end{aligned}
$$

If condition $\beta \leq k n$ is satisfied, then $k_{x}=\sqrt{(k n)^{2}-\beta^{2}}$ is a real value and each plane wave component is guided by waveguide, since factor $\exp \left[j\left(\sqrt{(n k)^{2}-\beta^{2}}\right) x\right]=\exp \left[j\left|k_{x}\right| x\right]$ is trigonometric function. If $\beta>k n$, then $k_{x}=\sqrt{(k n)^{2}-\beta^{2}}=\operatorname{Im}\left(\left|k_{x}\right|\right)$ is a imaginary value and factor $\exp \left[j\left(\sqrt{(n k)^{2}-\beta^{2}}\right) x\right]=\exp \left[-\left|k_{x}\right| x\right]$ represents decaying field or evanescent field along $\mathrm{x}$.

The index profile of a general five-layer slab is shown in Fig. 3-4. Channel one of the slab has refractive index $n_{c}$, channel two has refractive index $n_{r}, n_{c} \geq n_{r}$. All possible modes are guided modes in the first channel, since $\beta \leq k n_{c}$. From the point of view of the second channel however some modes are guided, $\beta \leq k n_{r}$ (case 1 in Fig. 3-4) and others are evanescent, $\beta>k n_{r}$ (case 2 in Fig. 3-4).

By analogy to the three-layer case (ref. [33]) we consider first channel only to develop the generic characteristic equation for propagation modes in this channel (and therefore in the whole slab). Two types of waves are present simultaneously, the one which has done one 
round trip already (round trip along width of the channel, along $\mathrm{x}$-axis) and the one which did not yet (see Fig. 3-5).

$$
\text { Case } 1 k n_{b} \leq \boldsymbol{\beta} \leq k n_{r}, k n_{C}
$$

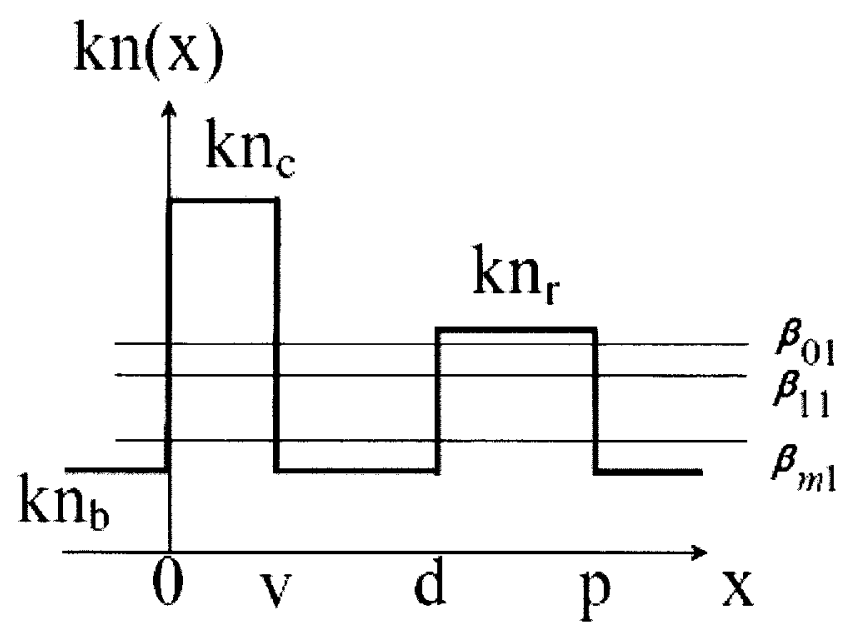

Case $2 k n_{b}, k n_{r} \leq \boldsymbol{\beta} \leq k n_{C}$

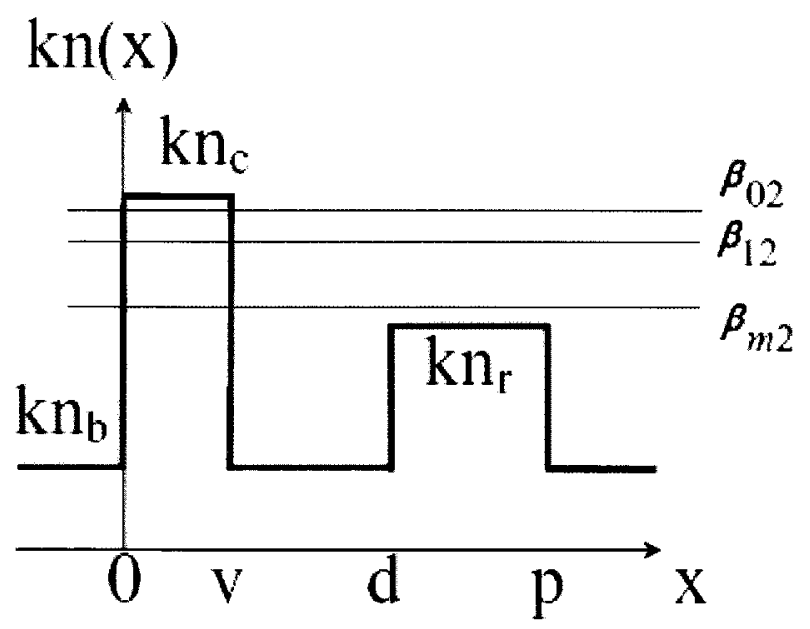

Fig. 3-4. Refractive index profile in 5-layer slab. Case 1, modes are guided in the second channel of the slab. Case 2 , modes are evanescent in the second channel of the slab. 


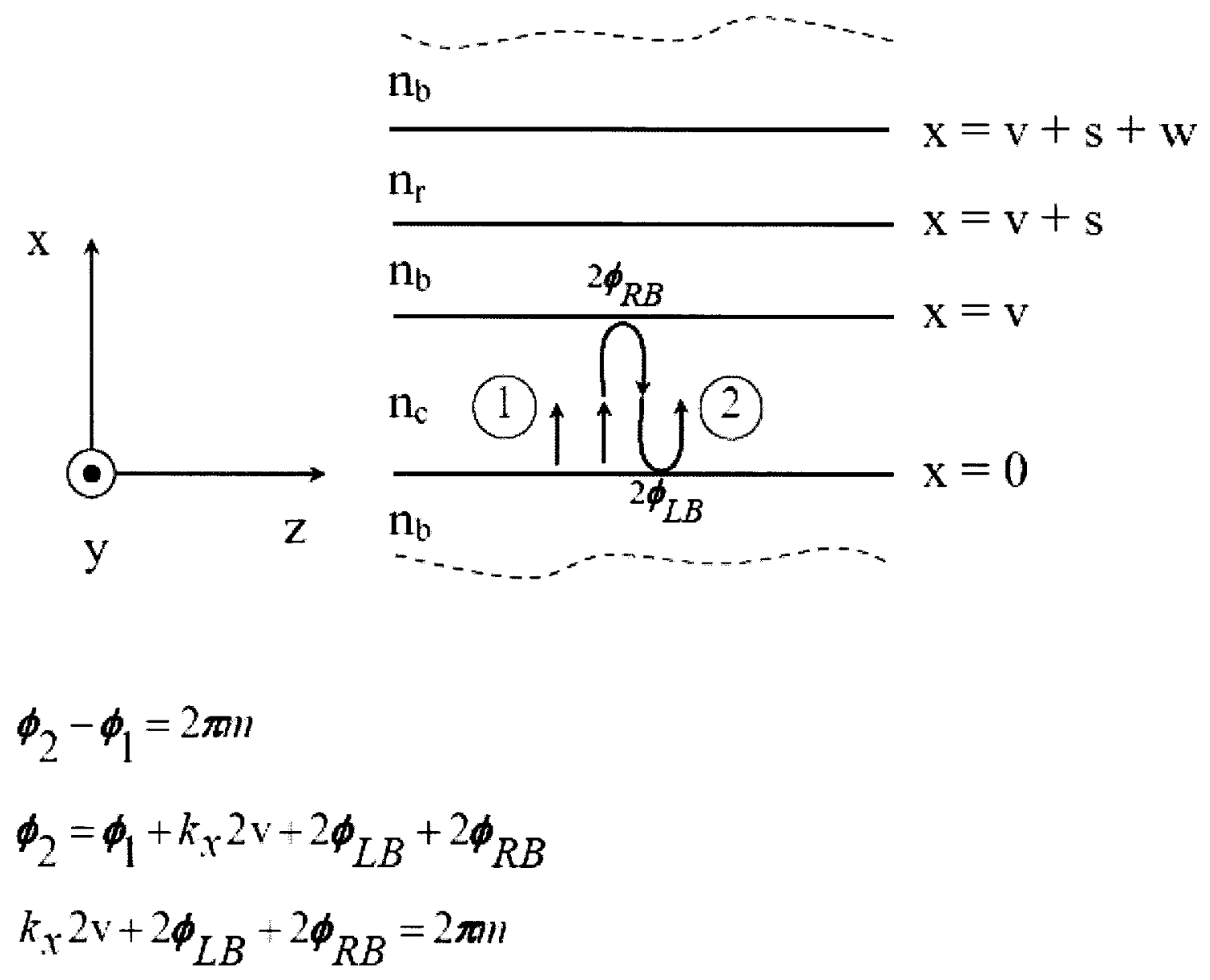

Fig. 3-5. One channel of the slab. Generic characteristic equation satisfying phase consistency conditions.

In order to be a guided mode, both types of waves have to interfere constructively. Therefore, the phase evolution corresponding to one round trip has to be a multiple of $2 \pi$. Based on such arguments, the characteristic equation is:

$k_{x} 2 \mathrm{v}+2 \phi_{L B}+2 \phi_{R B}=2 \pi m$ 
The left hand side of this equation is the total phase which mode acquired after one round trip in the channel. The first term, $k_{x} 2 \mathrm{v}$ is the phase evolution due to propagation distance $2 \mathrm{v}$ along $\mathrm{x}$-axis. $\phi_{L B}$ is the half phase shift introduced at the left boundary upon reflection and $\phi_{R B}$ is the half phase shift introduced at the right boundary upon reflection. Unlike the threelayer case, where $\phi_{R B}$ and $\phi_{L B}$ are the well known Goos-Hanschen shifts, here they depend on the refractive index profile of the whole structure including indices of each layer. We define an effective index $n_{\text {eff }}$ to characterize the phase velocity of the mode along $\vec{z}$ :

$k_{z}=\beta=k n_{e f f}$

$k_{x}^{2}+k_{z}^{2}=\left(k n_{c}\right)^{2}$

$k_{x}=k \sqrt{n_{c}^{2}-n_{e f f}^{2}}$

$k \mathrm{v} \sqrt{n_{c}^{2}-n_{e f f}^{2}}+\phi_{L B}+\phi_{R B}=m \pi$

With appropriate substitution, (in order to simplify further derivations)

$2 \phi_{L B} \rightarrow 2 \pi-2 \phi_{L B}$

$2 \phi_{R B} \rightarrow 2 \pi-2 \phi_{R B}$

$m \rightarrow m+2$

the equation (3-27) can be brought into the form:

$k_{x} \mathrm{v}=\phi_{L B}+\phi_{R B}+\pi m$

The above equation is the eigenvalue equation for a five-layer slab waveguide where the actual functional dependence for the phase shifts, $\phi_{R B}=\phi_{R B}(\beta)$ and $\phi_{L B}=\phi_{L B}(\beta)$ depend on all the parameters of the five-layer structure. Consider each case in details.

Case 1, the mode is guided in both channels:

$k n_{b} \leq \beta \leq k n_{r}, k n_{c}$

Define the effective refractive index (modal refractive index) as:

$\beta=k n_{\text {eff }}$

Introduce new variables: 
$u_{c}^{2}=k^{2} n_{c}^{2}-\beta^{2}=k^{2}\left(n_{c}^{2}-n_{e f f}^{2}\right) \geq 0$

$u_{r}^{2}=k^{2} n_{r}^{2}-\beta^{2}=k^{2}\left(n_{r}^{2}-n_{e f f}^{2}\right) \geq 0$

$u_{b}^{2}=\beta^{2}-k^{2} n_{b}^{2}=k^{2}\left(n_{e f f}^{2}-n_{b}^{2}\right) \geq 0$

Then, in each region of the slab coupler the general solution is as follows:

1) $x<0$

$$
e_{y}=A_{1} \exp \left[u_{b} x\right]
$$

since $e_{y}(x \rightarrow-\infty) \rightarrow 0$

$$
h_{z}=\frac{j}{\omega \mu} u_{b} A_{1} \exp \left[u_{b} x\right]
$$

2) $0<x<v$

We express fields in this region through the phase at the left boundary, $\phi_{L B}$ or separately through the phase at the right boundary, $\phi_{R B}$

$$
\begin{aligned}
& e_{y}=A_{2} \cos \left(u_{c} x-\phi_{L B}\right) \\
& h_{z}=\frac{j}{\omega \mu}(-1) u_{c} A_{2} \sin \left(u_{c} x-\phi_{L B}\right) \\
& e_{y}=A_{2} \cos \left(u_{c}(x-\mathrm{v})+\phi_{R B}\right) \\
& h_{z}=\frac{j}{\omega \mu}(-1) u_{c} A_{2} \sin \left(u_{c}(x-\mathrm{v})+\phi_{R B}\right)
\end{aligned}
$$

3) $\mathrm{v}<x<\mathrm{d}$

$$
\begin{aligned}
& e_{y}=A_{3} \cosh \left[u_{b}(x-\mathrm{v})+\phi_{3}\right] \\
& h_{z}=\frac{j}{\omega \mu} u_{b} A_{3} \sinh \left[u_{b}(x-\mathrm{v})+\phi_{3}\right]
\end{aligned}
$$

that was for even modes, and

$$
e_{y}=A_{3} \sinh \left[u_{b}(x-\mathrm{v})+\phi_{3}\right]
$$


$h_{z}=\frac{j}{\omega \mu} u_{b} A_{3} \cosh \left[u_{b}(x-\mathrm{v})+\phi_{3}\right]$

for odd modes

4) $\mathrm{d}<x<\mathrm{p}$

$$
\begin{aligned}
& e_{y}=A_{4} \cos \left(u_{r}(x-\mathrm{d})+\phi_{4}\right) \\
& h_{z}=\frac{j}{\omega \mu}(-1) u_{r} A_{4} \sin \left(u_{r}(x-\mathrm{d})+\phi_{4}\right)
\end{aligned}
$$

5) $x>\mathrm{p}$

$$
\begin{aligned}
& e_{y}=A_{5} \exp \left[-u_{b}(x-\mathrm{p}) x\right] \\
& h_{z}=\frac{j}{\omega \mu}(-1) u_{b} A_{5} \exp \left[-u_{b}(x-\mathrm{p})\right]
\end{aligned}
$$

There are 9 unknowns $\left(A_{1}, A_{2}, A_{3}, A_{4}, A_{5}, \beta, \phi_{L B}, \phi_{3}, \phi_{4}\right)$ and 8 equations from the 4 boundary conditions of the 2 tangential fields. The last unknown, for instance $A_{1}$ is related to the total power currying by the mode. Dispersion equation is additional equation for $\phi_{R B}$ :

$$
\phi_{R B}=\phi_{R B}\left(\phi_{L B}, \beta\right)
$$

At the each interface of the adjacent layers the tangential field components are continuous: $x=0$

$$
\begin{aligned}
& e_{y}: A_{1}=A_{2} \cos \left(-\phi_{L B}\right) \\
& h_{z}: u_{b} A_{1}=(-1) u_{c} A_{2} \sin \left(-\phi_{L B}\right)
\end{aligned}
$$

$x=\mathrm{v}$ for even modes:

$$
\begin{aligned}
& e_{y}: A_{2} \cos \left(\phi_{R B}\right)=A_{3} \cosh \left[\phi_{3}\right] \\
& h_{z}:-u_{c} A_{2} \sin \left(\phi_{R B}\right)=u_{b} A_{3} \sinh \left[\phi_{3}\right]
\end{aligned}
$$

for odd modes:

$$
\begin{aligned}
& e_{y}: A_{2} \cos \left(\phi_{R B}\right)=A_{3} \sinh \left[\phi_{3}\right] \\
& h_{z}:-u_{c} A_{2} \sin \left(\phi_{R B}\right)=u_{b} A_{3} \cosh \left[\phi_{3}\right]
\end{aligned}
$$


$x=\mathrm{d}$ for even modes:

$$
\begin{aligned}
& e_{y}: A_{3} \cosh \left[u_{b} \mathrm{~s}+\phi_{3}\right]=A_{4} \cos \left(\phi_{4}\right) \\
& h_{z}: u_{b} A_{3} \sinh \left[u_{b} \mathrm{~s}+\phi_{3}\right]=-u_{r} A_{4} \sin \left(\phi_{4}\right)
\end{aligned}
$$

where $\mathrm{s}=\mathrm{d}-\mathrm{v}$

for odd modes:

$$
\begin{aligned}
& e_{y}: A_{3} \sinh \left[u_{b} \mathrm{~s}+\phi_{3}\right]=A_{4} \cos \left(\phi_{4}\right) \\
& h_{z}: u_{b} A_{3} \cosh \left[u_{b} \mathrm{~s}+\phi_{3}\right]=-u_{r} A_{4} \sin \left(\phi_{4}\right)
\end{aligned}
$$

$x=\mathrm{p}$

$$
\begin{aligned}
& e_{y}: A_{4} \cos \left(u_{r} \mathrm{w}+\phi_{4}\right)=A_{5} \\
& h_{z}:-u_{r} A_{4} \sin \left(u_{r} \mathrm{w}+\phi_{4}\right)=-u_{b} A_{5}
\end{aligned}
$$

where $w=p-d$

By equating fields expressed through the phase $\phi_{L B}$ and $\phi_{R B}$ in region $0<x<\mathrm{v}$ we get characteristic equation for propagation modes:

$$
\begin{array}{ll}
e_{y}: & A_{0} \cos \left(u_{c} x-\phi_{L B}\right)=A_{0} \cos \left(u_{c}(x-\mathrm{v})+\phi_{R B}\right) \\
h_{z}:(-1) u_{c} A_{0} \sin \left(u_{c} x-\phi_{L B}\right)=(-1) u_{c} A_{0} \sin \left(u_{c}(x-\mathrm{v})+\phi_{R B}\right) \\
x=\mathrm{v} \quad \\
\quad \cos \left(u_{c} \mathrm{v}-\phi_{L B}\right)=\cos \left(\phi_{R B}\right) \\
\quad \sin \left(u_{c} \mathrm{v}-\phi_{L B}\right)=\sin \left(\phi_{R B}\right) \\
\Rightarrow \quad \tan \left(u_{c} \mathrm{v}-\phi_{L B}\right)=\tan \left(\phi_{R B}\right) \\
u_{c} \mathrm{v}=\phi_{L B}+\phi_{R B}+m \pi
\end{array}
$$

where $m=0,1,2, \cdots$ is the mode number.

After eliminating $A_{1}, A_{2}$ from (3-52), (3-53) we get condition for phase $\phi_{L B}$ : 
$u_{b}=u_{c} \tan \left(\phi_{L B}\right)$

$\phi_{L B}=\tan ^{-1}\left(\frac{u_{b}}{u_{c}}\right)$

Solving (3-54a, b), (3-55a, b) for phase $\phi_{R B}$ :

Even modes, $\tan \left(\phi_{R B}\right)=-\frac{u_{b}}{u_{c}} \tanh \left[\phi_{3}\right]$

Odd modes, $\tan \left(\phi_{R B}\right)=-\frac{u_{b}}{u_{c}} \operatorname{coth}\left[\phi_{3}\right]$

Solving (3-56a, b), (3-57a, b) for phase $\phi_{3}$ :

Even modes, $\tanh \left[u_{b} s+\phi_{3}\right]=-\frac{u_{r}}{u_{b}} \tan \left(\phi_{4}\right)$

Odd modes, $\operatorname{coth}\left[u_{b} s+\phi_{3}\right]=-\frac{u_{r}}{u_{b}} \tan \left(\phi_{4}\right)$

Solving (3-58), (3-59) for phase $\phi_{4}$ :

$\tan \left(u_{r} \mathrm{w}+\phi_{4}\right)=\frac{u_{b}}{u_{r}}$

Finally, we solve (3-68a, b), (3-69a, b), (3-70) for $\phi_{R B}$ and use it in (3-65) to get the characteristic equation involving only the propagation constant:

- for even modes,

$$
u_{c} \mathrm{v}=\tan ^{-1}\left(\frac{u_{b}}{u_{c}}\right)+\tan ^{-1}\left\{\frac{u_{b}}{u_{c}} \tanh \left[\tanh ^{-1}\left\{\frac{u_{r}}{u_{b}} \tan \left(\tan ^{-1}\left\{\frac{u_{b}}{u_{r}}\right\}-u_{r} \mathrm{w}\right)\right\}+u_{b} \mathrm{~s}\right]\right\}+m \pi
$$

where $u_{c}$ can be expressed in terms of $n_{\text {eff }}$ through: 
$n_{\text {eff }}=\sqrt{n_{c}^{2}-\left(\frac{u_{c}}{k}\right)^{2}}=\sqrt{n_{c}^{2}-\left(\frac{u_{c} \mathrm{v}}{k \mathrm{v}}\right)^{2}}$

$n_{e f f}=\sqrt{n_{c}^{2}-\left(\frac{\tan ^{-1}\left(\frac{u_{b}}{u_{c}}\right)+\tan ^{-1}\left\{\frac{u_{b}}{u_{c}} \tanh \left[\tanh ^{-1}\left\{\frac{u_{r}}{u_{b}} \tan \left(\tan ^{-1}\left\{\frac{u_{b}}{u_{r}}\right\}-u_{r} \mathrm{~W}\right)\right\}+u_{b} \mathrm{~s}\right]\right\}+m \pi}{k \mathrm{v}}\right)^{2}}$

$n_{\text {eff }}=G_{\text {even }}\left(n_{\text {eff }}, n_{r}, n_{c}, n_{b}, \mathrm{v}, \mathrm{w}, \mathrm{s}, \lambda\right)=G_{\text {even }}\left(n_{\text {eff }}, n_{r}, p\right)$

where,

$p$ is the set of parameters $n_{c}, n_{b}, \mathrm{v}, \mathrm{w}, \mathrm{s}, \lambda$

$G_{\text {even }}\left(n_{\text {eff }}, n_{r}, p\right)=\operatorname{RHS}(3-73)$

- for odd modes,

$u_{c} \mathrm{~V}=\tan ^{-1}\left(\frac{u_{b}}{u_{c}}\right)+\tan ^{-1}\left\{\frac{u_{b}}{u_{c}} \operatorname{coth}\left[\operatorname{coth}^{-1}\left\{\frac{u_{r}}{u_{b}} \tan \left(\tan ^{-1}\left\{\frac{u_{b}}{u_{r}}\right\}-u_{r} \mathrm{w}\right)\right\}+u_{b} \mathrm{~s}\right]\right\}+m \pi$

$n_{e f f}=\sqrt{n_{c}^{2}-\left(\frac{\tan ^{-1}\left(\frac{u_{b}}{u_{c}}\right)+\tan ^{-1}\left\{\frac{u_{b}}{u_{c}} \operatorname{coth}\left[\operatorname{coth}^{-1}\left\{\frac{u_{r}}{u_{b}} \tan \left(\tan ^{-1}\left\{\frac{u_{b}}{u_{r}}\right\}-u_{r} \mathrm{~W}\right)\right\}+u_{b} \mathrm{~s}\right]\right\}+m \pi}{k \mathrm{v}}\right)^{2}}$

$n_{\text {eff }}=G_{\text {odd }}\left(n_{\text {eff }}, n_{r}, p\right)$

where,

$G_{o d d}\left(n_{\text {eff }}, n_{r}, p\right)=\operatorname{RHS}(3-76)$ 
Case 2, the mode is evanescent in the second channel:

$$
k n_{b}, k n_{r} \leq \beta \leq k n_{c}
$$

compared to the previous case the variable $u_{r}$ becomes now complex:

$u_{r}^{2}=k^{2} n_{r}^{2}-\beta^{2}=k^{2}\left(n_{r}^{2}-n_{e f f}^{2}\right) \leq 0$

$u_{r}=j \gamma$, where $\gamma$ is real.

The fields are different from case 1 only in the second channel of the slab coupler: $\mathrm{d}<x<\mathrm{p}$

$$
\begin{aligned}
& e_{y}=A_{4} \cosh \left[\gamma(x-\mathrm{d})+\psi_{4}\right] \\
& h_{z}=\frac{j}{\omega \mu} \gamma A_{4} \sinh \left[\gamma(x-\mathrm{d})+\psi_{4}\right]
\end{aligned}
$$

Now we are going to work through several simplifications of these equations.

Because of the following identities:

$$
\begin{aligned}
& \cos (j \theta)=\cosh [\theta] \\
& \sin (j \theta)=j \sinh [\theta] \\
& \tan (j \theta)=j \tanh [\theta] \\
& \tan ^{-1}(j \theta)=j \tanh ^{-1}[\theta] \\
& \cosh [j \theta]=\cos (\theta) \\
& \sinh [j \theta]=j \sin (\theta) \\
& \tanh [j \theta]=j \tan (\theta) \\
& \tanh { }^{-1}[j \theta]=j \tan ^{-1}(\theta),
\end{aligned}
$$

all equations of case 1 can be used without any modification since when the variable $u_{r}$ becomes complex, the fields in the second channel automatically convert into their appropriate form:

$e_{y}=A_{4} \cos \left(u_{r}(x-\mathrm{d})+\phi_{4}\right)=A_{4} \cos \left(j \gamma(x-\mathrm{d})+j \psi_{4}\right)=$ 


$$
\begin{aligned}
& =A_{4} \cosh \left[\gamma(x-\mathrm{d})+\psi_{4}\right] \\
& h_{z}=\frac{j}{\omega \mu}(-1) u_{r} A_{4} \sin \left(u_{r}(x-\mathrm{d})+\phi_{4}\right)=\frac{j}{\omega \mu}(-1) j \gamma A_{4} \sin \left(j \gamma(x-\mathrm{d})+j \psi_{4}\right)= \\
& =\frac{j}{\omega \mu}(-1) j \gamma A_{4} j \sinh \left[\gamma(x-\mathrm{d})+\psi_{4}\right]=\frac{j}{\omega \mu} \gamma A_{4} \sinh \left[\gamma(x-\mathrm{d})+\psi_{4}\right] \\
& u_{c} \mathrm{v}=\tan ^{-1}\left(\frac{u_{b}}{u_{c}}\right)+\tan ^{-1}\left\{\frac{u_{b}}{u_{c}} \tanh \left[\tanh ^{-1}\left\{\frac{\gamma}{u_{b}} \tanh \left(\tanh ^{-1}\left\{\frac{u_{b}}{\gamma}\right\}+\gamma \mathrm{w}\right)\right\}+u_{b} \mathrm{~s}\right]\right\}+m \pi
\end{aligned}
$$

Therefore, if we use the textual substitution, $u_{r}=j \gamma$ in (3-71) and the above identities, we get exactly the same result:

$$
\begin{aligned}
& u_{c} \mathrm{v}=\tan ^{-1}\left(\frac{u_{b}}{u_{c}}\right)+\tan ^{-1}\left\{\frac{u_{b}}{u_{c}} \tanh \left[\tanh ^{-1}\left\{\frac{u_{r}}{u_{b}} \tan \left(\tan ^{-1}\left\{\frac{u_{b}}{u_{r}}\right\}-u_{r} \mathrm{w}\right)\right\}+u_{b} \mathrm{~s}\right]\right\}+m \pi \\
& =\tan ^{-1}\left(\frac{u_{b}}{u_{c}}\right)+\tan ^{-1}\left\{\frac{u_{b}}{u_{c}} \tanh \left[\tanh ^{-1}\left\{\frac{j \gamma}{u_{b}} \tan \left(\tan ^{-1}\left\{\frac{u_{b}}{j \gamma}\right\}-j \gamma \mathrm{w}\right)\right\}+u_{b} \mathrm{~s}\right]\right\}+m \pi \\
& =\tan ^{-1}\left(\frac{u_{b}}{u_{c}}\right)+\tan ^{-1}\left\{\frac{u_{b}}{u_{c}} \tanh \left[\tanh ^{-1}\left\{\frac{j \gamma}{u_{b}} \tan \left(\tan ^{-1}\left\{-j \frac{u_{b}}{\gamma}\right\}-j \gamma \mathrm{w}\right)\right\}+u_{b} \mathrm{~s}\right]\right\}+m \pi \\
& =\tan ^{-1}\left(\frac{u_{b}}{u_{c}}\right)+\tan ^{-1}\left\{\frac{u_{b}}{u_{c}} \tanh \left[\tanh ^{-1}\left\{\frac{j \gamma}{u_{b}} \tan \left(-j \tanh ^{-1}\left\{\frac{u_{b}}{\gamma}\right\}-j \gamma \mathrm{w}\right)\right\}+u_{b} \mathrm{~s}\right]\right\}+m \pi \\
& =\tan ^{-1}\left(\frac{u_{b}}{u_{c}}\right)+\tan ^{-1}\left\{\frac{u_{b}}{u_{c}} \tanh \left[\tanh ^{-1}\left\{\frac{j \gamma}{u_{b}}(-j) \tanh \left(\tanh ^{-1}\left\{\frac{u_{b}}{\gamma}\right\}+\gamma \mathrm{w}\right)\right\}+u_{b} \mathrm{~s}\right]\right\}+m \pi \\
& =\tan ^{-1}\left(\frac{u_{b}}{u_{c}}\right)+\tan ^{-1}\left\{\frac{u_{b}}{u_{c}} \tanh \left[\tanh ^{-1}\left\{\frac{\gamma}{u_{b}} \tanh \left(\tanh ^{-1}\left\{\frac{u_{b}}{\gamma}\right\}+\gamma \mathrm{w}\right)\right\}+u_{b} \mathrm{~s}\right]\right\}+m \pi
\end{aligned}
$$

This means that the characteristic equations (3-71), (3-75) actually covers both cases: 
case $1 k n_{b} \leq \beta \leq k n_{r}, k n_{c}$ and

case $2 k n_{b}, k n_{r} \leq \beta \leq k n_{c}$. We do not have to distinguish them. When $n_{r}$ varies from $n_{b}$ to $n_{c}$, the variable $u_{r}$ as defined above varies from pure imaginary to pure real, it never has both parts (real and imaginary) simultaneously.

Now, we investigate a difference between the even and the odd modes. When the second channel is a guiding channel $\left(k n_{b} \leq \beta \leq k n_{r}, k n_{c}\right)$ there are two possibilities: the field in the first channel at $x=\mathrm{v}$ and field in the second channel at $x=\mathrm{d}$ can be of the same sign or of opposite sign. Both possibilities are illustrated in Fig. 3-6a, b.

When signs are the same:

$$
\left\{\begin{array} { l } 
{ E ( x = \mathrm { v } ) > 0 } \\
{ E ( x = \mathrm { d } ) > 0 }
\end{array} \quad \text { or } \quad \left\{\begin{array}{l}
E(x=\mathrm{v})<0 \\
E(x=\mathrm{d})<0
\end{array}\right.\right.
$$

the evanescent field in between the two channels has no crossing zero-point and therefore must be described by a hyperbolic cosine function, $\cosh (x)$.

When signs are opposite:

$\left\{\begin{array}{l}E(x=\mathrm{v})<0 \\ E(x=\mathrm{d})>0\end{array} \quad\right.$ or $\quad\left\{\begin{array}{l}E(x=\mathrm{v})>0 \\ E(x=\mathrm{d})<0\end{array}\right.$

the evanescent field in between two channels does have a crossing zero-point and therefore must be described by hyperbolic sine function, $\sinh (x)$.

We can combine the even and the odd modes into a single unified expression. Consider equation (3-69a). The right hand side in general can be greater than unity, however the left hand side cannot (see graph $\tanh (x)$ in Fig. 3-7). If that happens, then $\tanh (x)$ should be replaced by $\frac{1}{\tanh (x)}$, i.e. by $\operatorname{coth}(x)$. The last one is in fact equation (3-69b). 


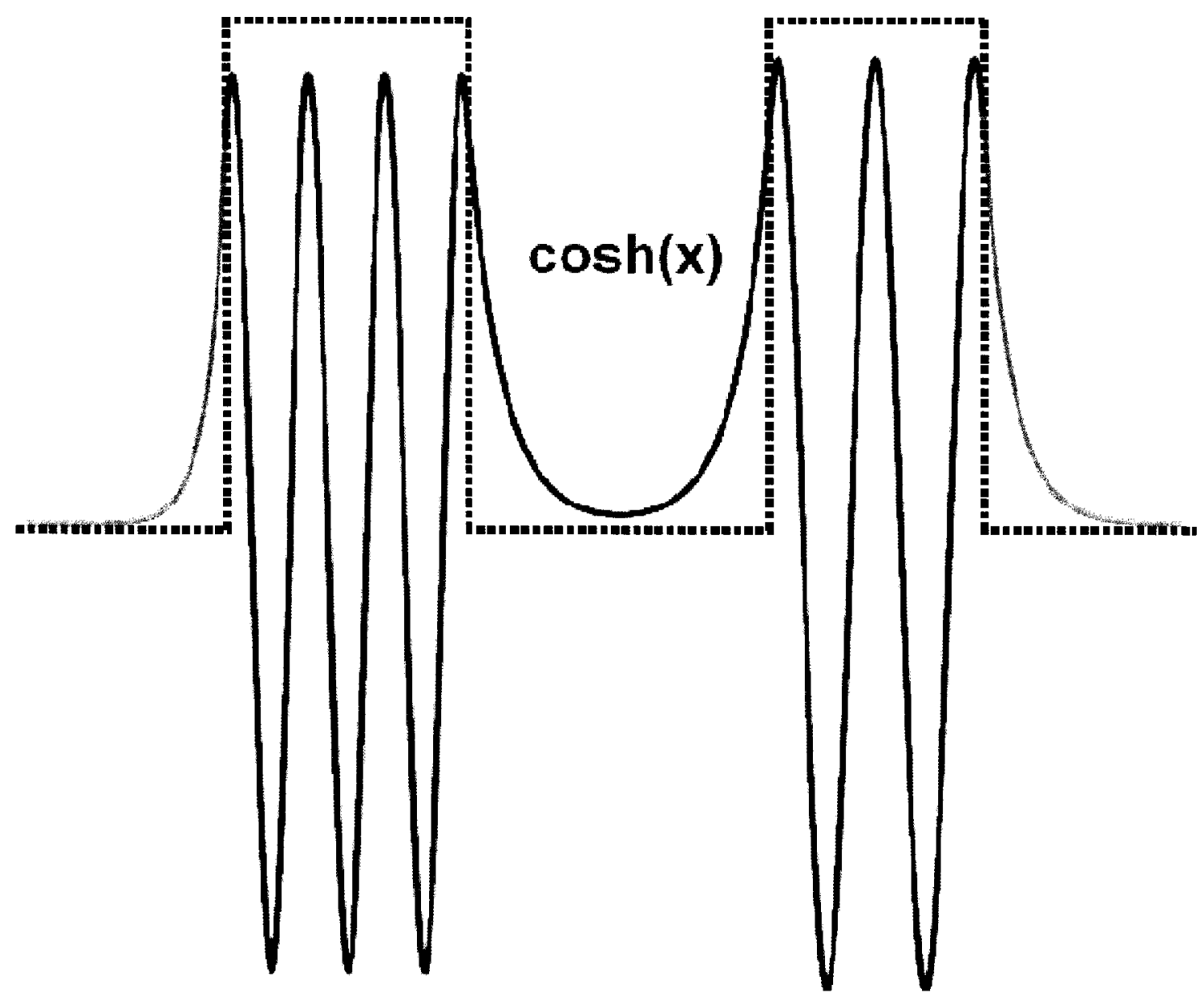

Fig. 3-6a. Electric field distribution between two channels for even mode. 


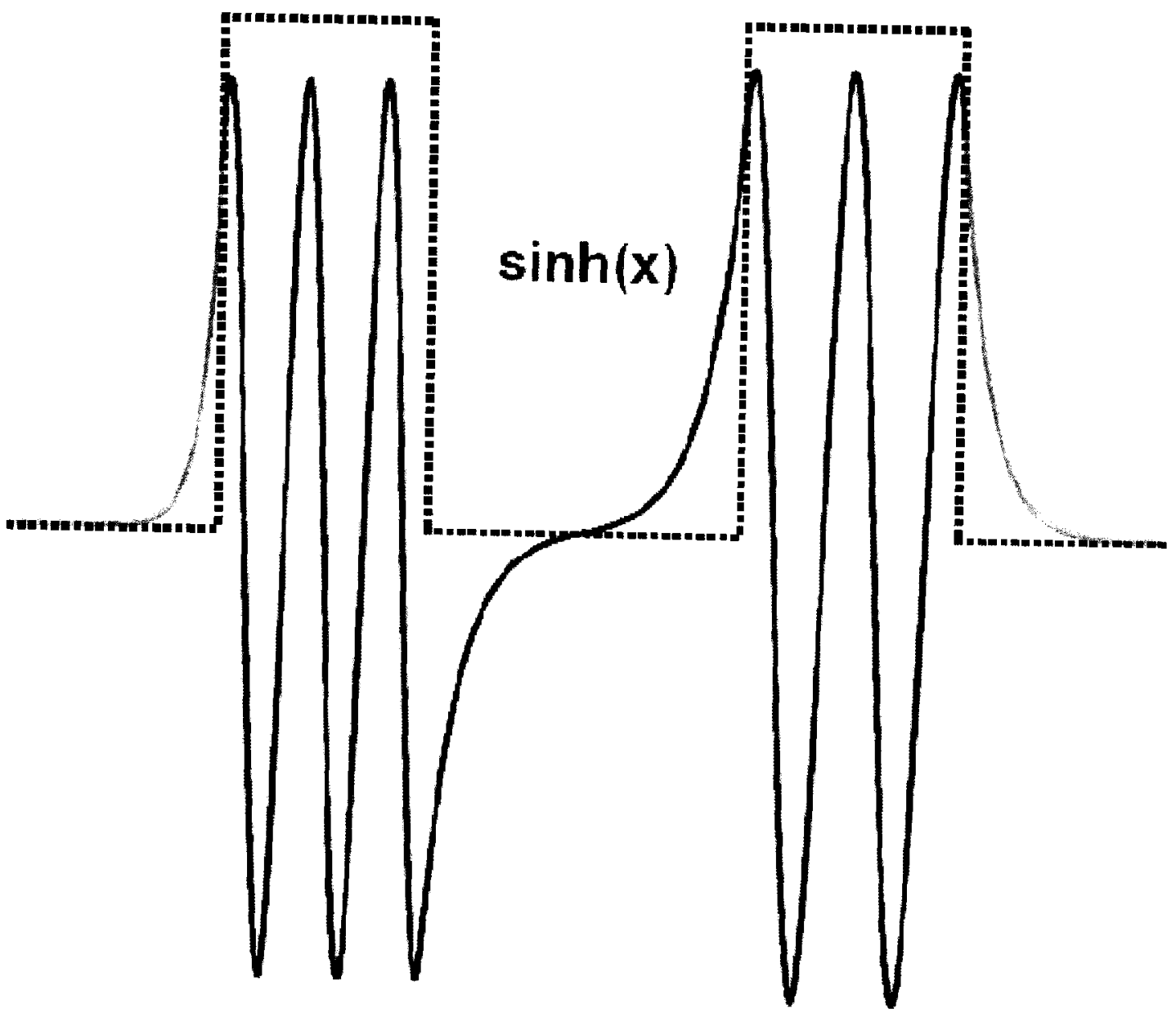

Fig. 3-6b. Electric field distribution between two channels for odd mode. 


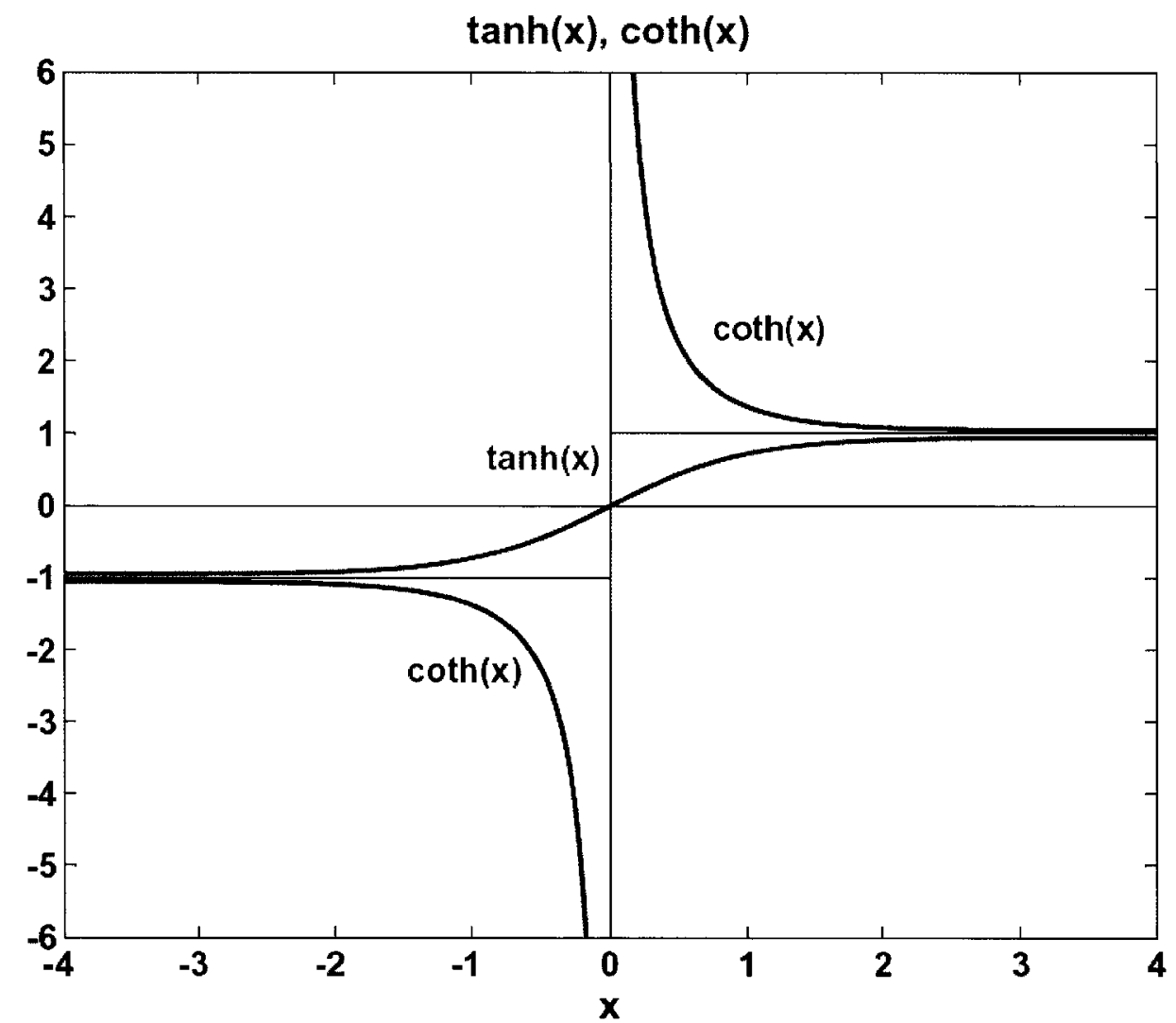

Fig. 3-7a. Hyperbolic functions, $\tanh (x), \operatorname{coth}(x)$. 


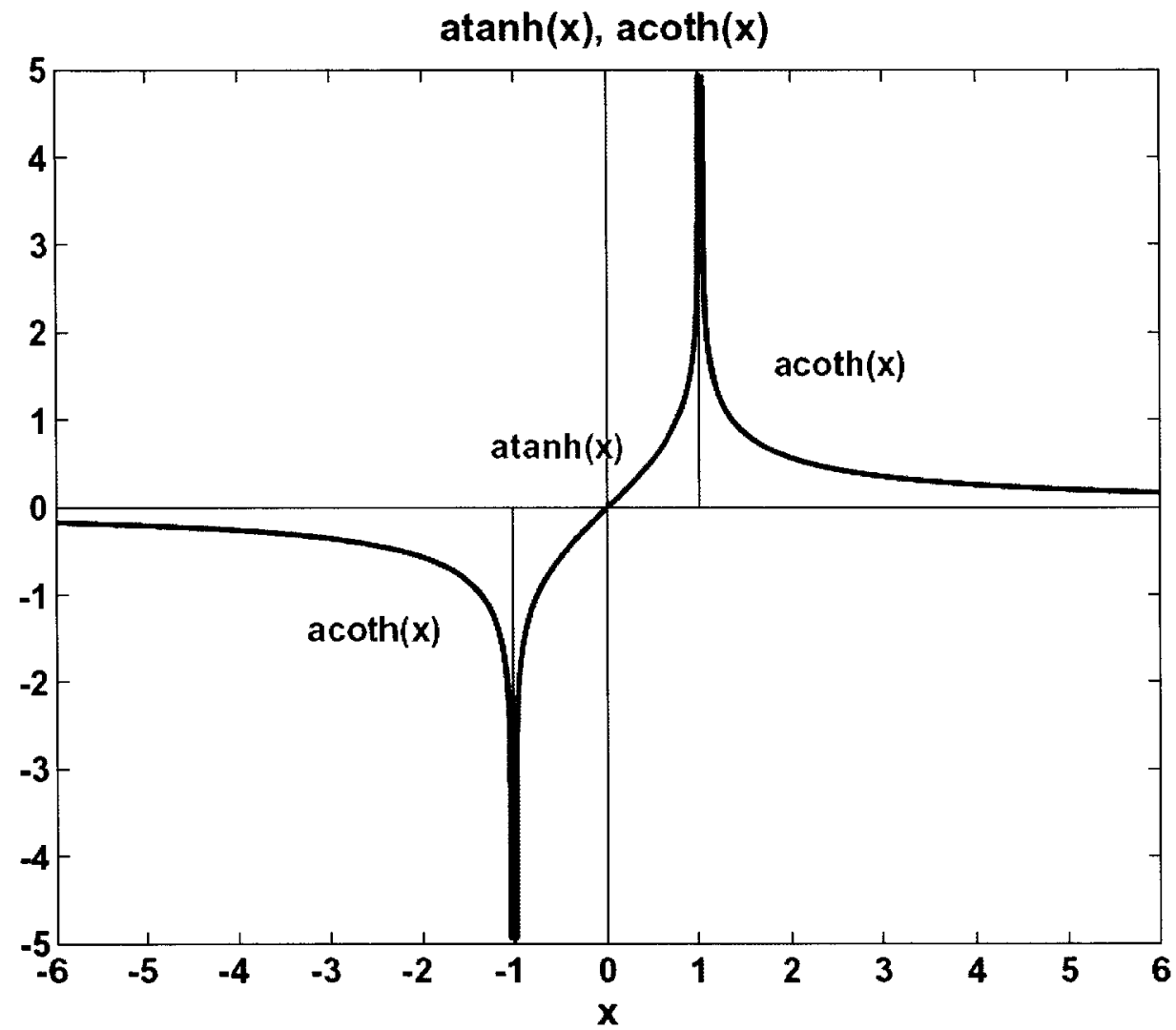

Fig. 3-7b. Inverse hyperbolic functions, $\arctan h(x), \operatorname{arccoth}(x)$.

To formally combine case when mode is even and case when mode is odd we can simply eliminate $\phi_{3}$ in $(3-68 \mathrm{a}, \mathrm{b}),(3-69 \mathrm{a}, \mathrm{b})$ :

1) even modes:

$$
\begin{aligned}
& \tanh \left[u_{b} s+\phi_{3}\right]=-\frac{u_{r}}{u_{b}} \tan \left(\phi_{4}\right) \\
& \frac{\tanh \left[u_{b} s\right]+\tanh \left[\phi_{3}\right]}{1+\tanh \left[u_{b} s\right] \tanh \left[\phi_{3}\right]}=-\frac{u_{r}}{u_{b}} \tan \left(\phi_{4}\right)
\end{aligned}
$$


$\tanh \left[\phi_{3}\right]=\frac{-\frac{u_{r}}{u_{b}} \tan \left(\phi_{4}\right)-\tanh \left[u_{b} s\right]}{1+\frac{u_{r}}{u_{b}} \tan \left(\phi_{4}\right) \tanh \left[u_{b} s\right]}$

$\tan \left(\phi_{R B}\right)=-\frac{u_{b}}{u_{c}} \tanh \left[\phi_{3}\right]=\frac{u_{b}}{u_{c}} \cdot \frac{\frac{u_{r}}{u_{b}} \tan \left(\phi_{4}\right)+\tanh \left[u_{b} s\right]}{1+\frac{u_{r}}{u_{b}} \tan \left(\phi_{4}\right) \tanh \left[u_{b} s\right]}$

2) odd modes:

$$
\begin{aligned}
& \operatorname{coth}\left[u_{b} s+\phi_{3}\right]=-\frac{u_{r}}{u_{b}} \tan \left(\phi_{4}\right) \\
& \frac{1+\tanh \left[u_{b} s\right] \tanh \left[\phi_{3}\right]}{\tanh \left[u_{b} s\right]+\tanh \left[\phi_{3}\right]}=-\frac{u_{r}}{u_{b}} \tan \left(\phi_{4}\right) \\
& \tanh \left[\phi_{3}\right]=\frac{1+\frac{u_{r}}{u_{b}} \tan \left(\phi_{4}\right) \tanh \left[u_{b} s\right]}{-\frac{u_{r}}{u_{b}} \tan \left(\phi_{4}\right)-\tanh \left[u_{b} s\right]} \\
& \tan \left(\phi_{R B}\right)=-\frac{u_{b}}{u_{c}} \operatorname{coth}\left[\phi_{3}\right]=\frac{u_{b}}{u_{c}} \cdot \frac{u_{r}}{1+\frac{u_{b}}{u_{r}} \tan \left(\phi_{4}\right)+\tanh \left[\phi_{b} s\right] \tanh \left[u_{b} s\right]}
\end{aligned}
$$

So, after eliminating $\phi_{3}$ both cases are described by the same formula.

We can also eliminate $\tan \left(\phi_{4}\right)$ in (3-97), (3-101) with the help of (3-70) and obtain a universal formula for $\tan \left(\phi_{R B}\right)$,

$$
\tan \left[\phi_{4}\right]=\frac{\frac{u_{b}}{u_{r}}-\tan \left[u_{r} \mathrm{w}\right]}{1+\frac{u_{b}}{u_{r}} \tan \left[u_{r} \mathrm{w}\right]}
$$


$\tan \left[\phi_{R B}\right]=\frac{\frac{u_{b}}{u_{c}}-\frac{u_{r}}{u_{c}} \tan \left[u_{r} \mathrm{w}\right]+\frac{u_{b}}{u_{c}}\left(1+\frac{u_{b}}{u_{r}} \tan \left[u_{r} \mathrm{w}\right]\right) \tanh \left[u_{b} \mathrm{~s}\right]}{1+\frac{u_{b}}{u_{r}} \tan \left[u_{r} \mathrm{w}\right]+\left(1-\frac{u_{r}}{u_{b}} \tan \left[u_{r} \mathrm{w}\right]\right) \tanh \left[u_{b} \mathrm{~s}\right]}$

$n_{e f f}=\sqrt{n_{c}^{2}-\left(\frac{u_{c} \mathrm{v}}{k \mathrm{v}}\right)^{2}}=\sqrt{n_{c}^{2}-\left(\frac{\phi_{L B}+\phi_{R B}+m \pi}{k \mathrm{v}}\right)^{2}}$

$n_{\text {eff }}=G\left(n_{\text {eff }}\right)$

where,

$G\left(n_{\text {eff }}\right)=\operatorname{RHS}(3-104)$ is our 'simplified' transcendental equation for $n_{\text {eff }}$ of the modes of the five-layer slab, and $\phi_{R B}$ is found from (3-103), while $\phi_{L B}$ is found from (3-67).

\subsection{Simple analysis of the characteristic equation}

Using derived formulas (3-67) - (3-70) and graphs of hyperbolic and trigonometric functions (tanh, coth, atanh, acoth) we can predict the behavior of the propagation constant versus $n_{r}$. First we note from (3-71) that the phase factor due to reflection at the left border in the main channel, $\phi_{L B}=\tan ^{-1}\left(\frac{u_{b}}{u_{c}}\right)$ has no $n_{r}$-dependence, therefore $u_{c} \mathrm{v}$ depends on $n_{r}$ only through phase factor at the right border, $\phi_{R B}$. The function $\tan \left(\phi_{R B}\right)$ is periodic with poles at $\phi_{R B}= \pm \frac{\pi}{2}, \pm \frac{3 \pi}{2} \ldots$ When $\phi_{R B}$ can actually cross those poles ? If a mode is even, then (3-68a) is valid, its RHS (right hand side) proportional to $\tanh \left(\phi_{3}\right)$. It is bounded by values \pm 1 , $\left|\tanh \left(\phi_{3}\right)\right| \leq 1$, therefore $\tan \left(\phi_{R B}\right)$ can not reach $\pm \infty$ and can not go through the poles for even modes. For odd modes (3-75) is valid, the RHS of which is proportional to $\operatorname{coth}\left(\phi_{3}\right)$. That function has a pole at $\phi_{3}=0$ (graph is shown in the Fig. 3-7). Whenever $\operatorname{coth}\left(\phi_{3}\right)$ going through the pole $\phi_{3}=0, \tan \left(\phi_{R B}\right)$ crossing its own pole at $\phi_{R B}= \pm \frac{\pi}{2}, \pm \frac{3 \pi}{2} \ldots$ At this point 
we have to keep in mind that $\tan \left(\phi_{R B}\right)$ is periodic, so its proper inverse function must be multi-valued function (Fig. 3-8b). However, standard conventional inverse function of $\tan \left(\phi_{R B}\right)=y, \arctan (y)$ is single-valued, basically only one possible branch is used. That means, if $\tan \left(\phi_{R B}\right)$ going from $-\infty$ to $+\infty$, the standard $\arctan (y)=\phi_{R B}$ will experience a non-physical 'jump'. It is obvious, $\phi_{R B}$ must be continuous function, this is also seen from the multi-valued $\arctan (y)=\phi_{R B}$. To cancel that 'jump' we have to add $\pi$ every time $\tan \left(\phi_{R B}\right)$ crosses the pole.

Now we look closer at $n_{r}$-dependence. If $n_{r}$ is increasing, $n_{\text {eff }}$ is monotonically increasing (see Appendix A for details). Then $u_{c} \mathrm{v}=k \mathrm{v} \sqrt{n_{c}^{2}-n_{e f f}^{2}}$ is monotonically decreasing. As seen from (3-65) $\phi_{R B}$ is decreasing as well. For even modes, from (3-68a) $\phi_{3}$ must be increasing. When $\phi_{3}$ crosses zero, the derivative $\frac{d}{d \phi_{3}} \tanh \left(\phi_{3}\right)$ is the largest possible (see graph $\tanh (\mathrm{x})$ in Fig. 3-7). Therefore, if value $n_{r}$ is such that $\phi_{3}$ is near zero, then a small deviation $n_{r}$ from that value causes significant variation of $\tanh \left(\phi_{3}\right)$ and consequently of $\tan \left(\phi_{R B}\right), u_{c} \mathrm{v}$ and $n_{\text {eff }}$. That point (value $n_{r}$ ) is very important to identify, because it marks validity of perturbation approach. Before, and up until that point, perturbation theory is valid ( $n_{\text {eff }}$ grows slowly, i.e. $\frac{d n_{e f f}}{d n_{r}} \ll<1$, in the Fig. 3-10 it is a region before $\left.n_{r 0}\right)$. After that point it breaks down ( $n_{\text {eff }}$ grows fast, i.e. condition $\frac{d n_{\text {eff }}}{d n_{r}} \ll<1$ is not valid any more, in the Fig. $3-10$ it is a region between $n_{r 0}$ and $n_{r 1}$ ).

Now examine the odd mode, equation (3-68b). From the graph $\operatorname{cotanh}(x)$ we see that when $\phi_{3}$ crossing zero the derivative, $\frac{d}{d \phi_{3}} \operatorname{cotanh}\left(\phi_{3}\right)$ is large. Therefore, the corresponding value 
$n_{r}$, which makes $\phi_{3}$ equal to zero for the odd mode, is also a special point from perturbation theory approach.

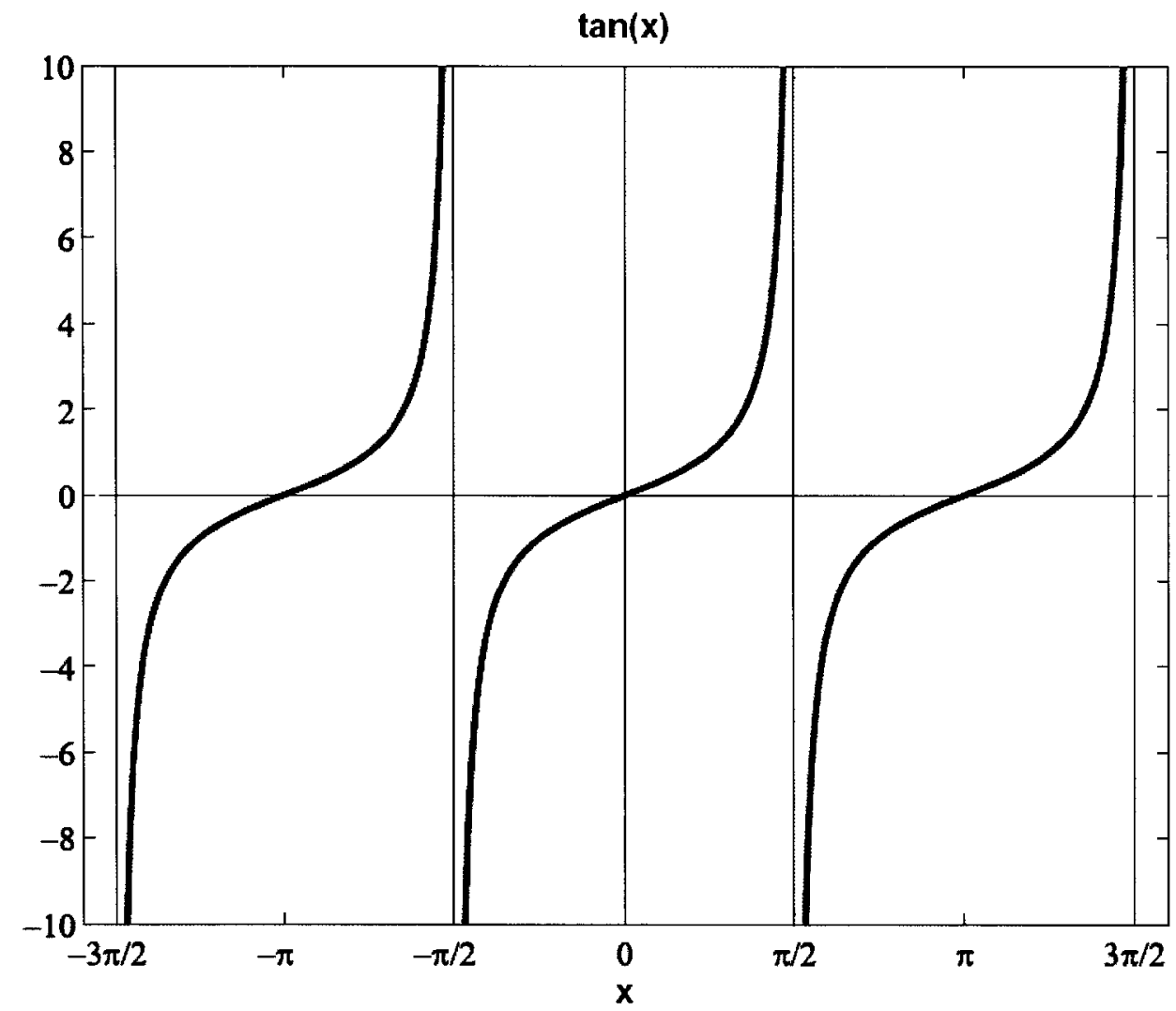

Fig. 3-8a. Trigonometric tangent function, $\tan (x)$. 


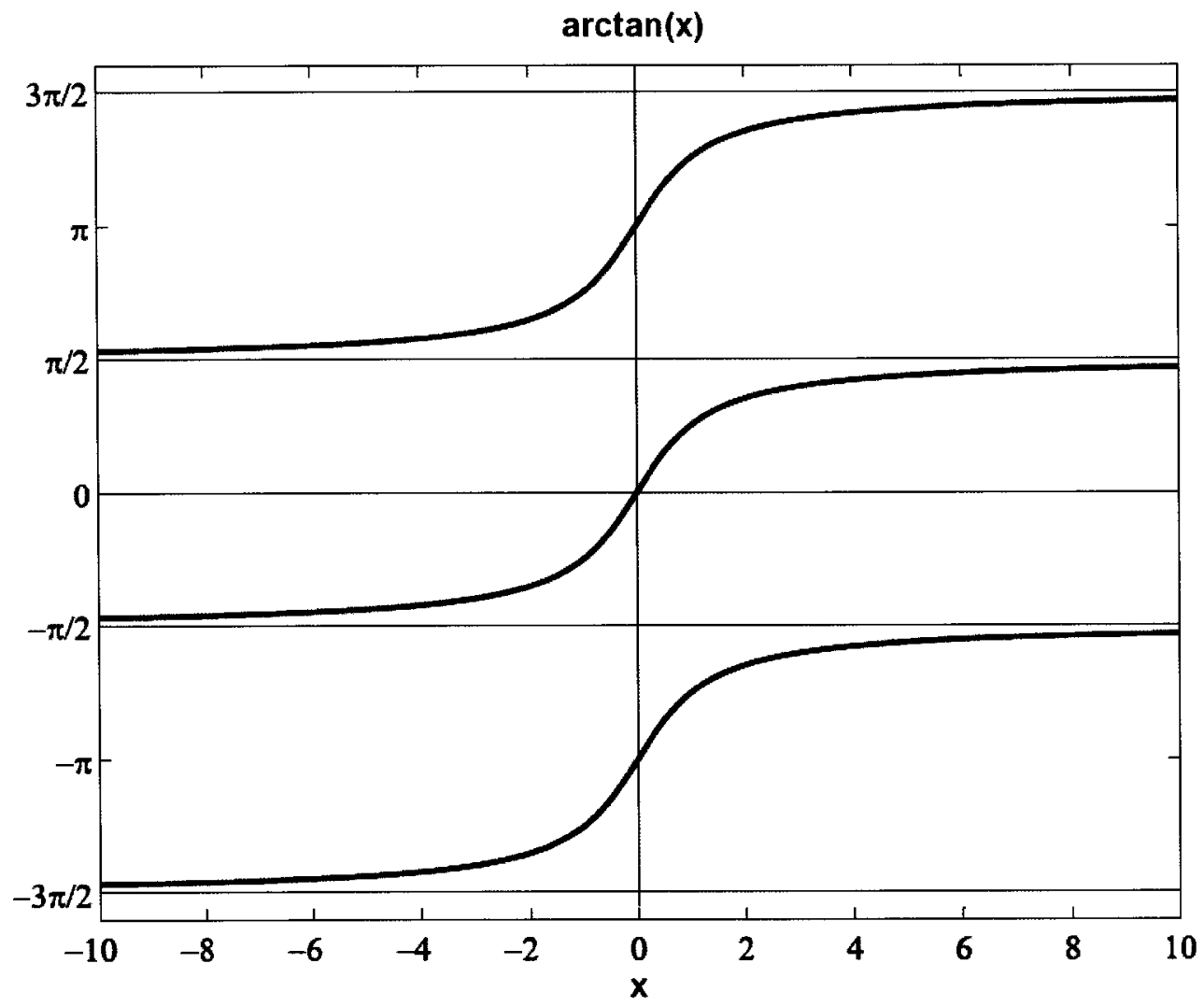

Fig. 3-8b. Proper inverse function of trigonometric tangent, $\tan ^{-1}(x)$ is multivalued function. Only one branch of it is used as a $\arctan (x)$. 
What quantity is responsible for switching between even and odd modes? The RHS of the equations (3-69a), (3-69b) is the same: $-\frac{u_{r}}{u_{b}} \tan \left(\phi_{4}\right)$. If its magnitude is greater than 1 , $\tanh \left(u_{b} s+\phi_{3}\right)$ does not exist and the even mode does not exist. So, condition for odd mode is $\left|-\frac{u_{r}}{u_{b}} \tan \left(\phi_{4}\right)\right|>1$. When $\left|-\frac{u_{r}}{u_{b}} \tan \left(\phi_{4}\right)\right|<1$, then $\operatorname{cotanh}\left(u_{b} \mathrm{~s}+\phi_{3}\right)$ does not exist, that condition for even mode. When $\left|-\frac{u_{r}}{u_{b}} \tan \left(\phi_{4}\right)\right|=1$, this is condition for switching. At this condition magnitude of $\phi_{3}$ is very large.

We see from (3-70), that phase factor $\phi_{4}$ depends on $n_{r}$ relatively slowly: $\phi_{4}=\arctan \left(\frac{u_{b}}{u_{r}}\right)-u_{r} \mathrm{w}$, that function does not have peaks or poles, this is what is meant by slowly.

We can draw a diagram which helps to visualize various conditions, see Fig. 3-9. Assume we started at even mode. When $\phi_{3}$ going towards zero, point 1 , propagation constant and $n_{\text {eff }}$ start to grow quickly. $\phi_{3}$ grows quickly as well. Then it reaches infinity, point 2 . At this point the switching condition is satisfied. The mode switches from even to odd. Now $\phi_{3}$ is dropping quickly. When it reaches zero, point 3 , it still continues to fall, but not so fast. Propagation constant and $n_{\text {eff }}$ changing slowly again. After point 3 perturbation theory is valid, as well as before point 1.

From this simple analysis we see, that special points to watch for are those when $\phi_{3}=0$ for even and odd modes.

Considering even mode and $\phi_{3}=0$, then (3-69a) becomes,

$$
\tanh \left[u_{b} s\right]=-\frac{u_{r}}{u_{b}} \tan \left(\phi_{4}\right)
$$

Find $\tan \left(\phi_{4}\right)$ from $(3-70)$, 


$$
\begin{gathered}
\tan \left(\phi_{4}\right)=\frac{\frac{u_{b}}{u_{r}}-\tan \left(u_{r} \mathrm{w}\right)}{1+\frac{u_{b}}{u_{r}} \tan \left(u_{r} \mathrm{w}\right)} \\
\tanh \left[u_{b} \mathrm{~s}\right]=\frac{\frac{u_{r}}{u_{b}} \tan \left(u_{r} \mathrm{w}\right)-1}{\frac{u_{b}}{u_{r}} \tan \left(u_{r} \mathrm{w}\right)+1}
\end{gathered}
$$

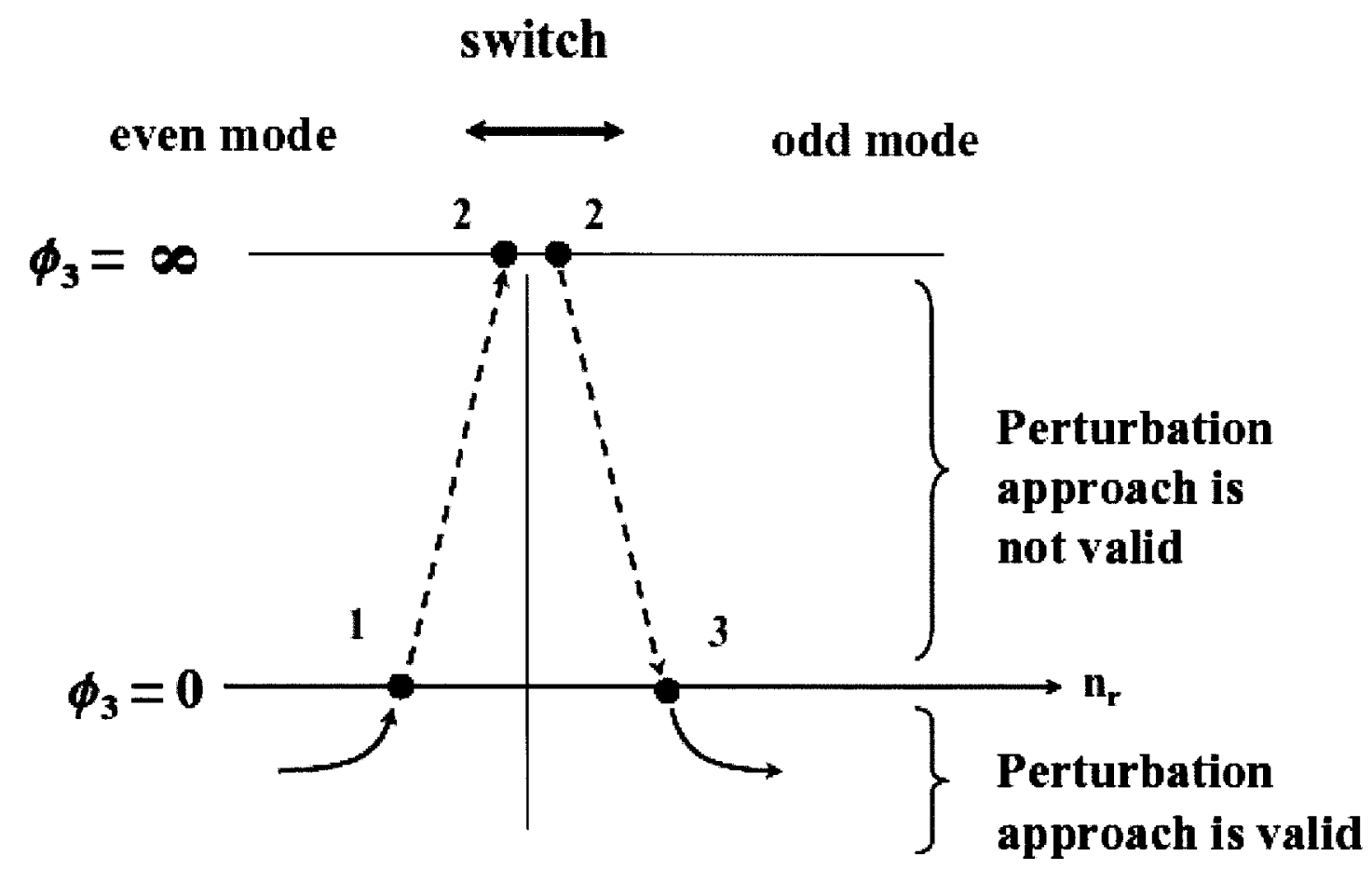

Fig. 3-9. $\quad$ Phase $\phi_{3}$ evolution diagram. Between $\phi_{3}=0$ and $\phi_{3}=\infty$ propagation constant, $\beta$ and modal index, $n_{\text {eff }}$ vary significantly, perturbation theory is not valid in this regime. 


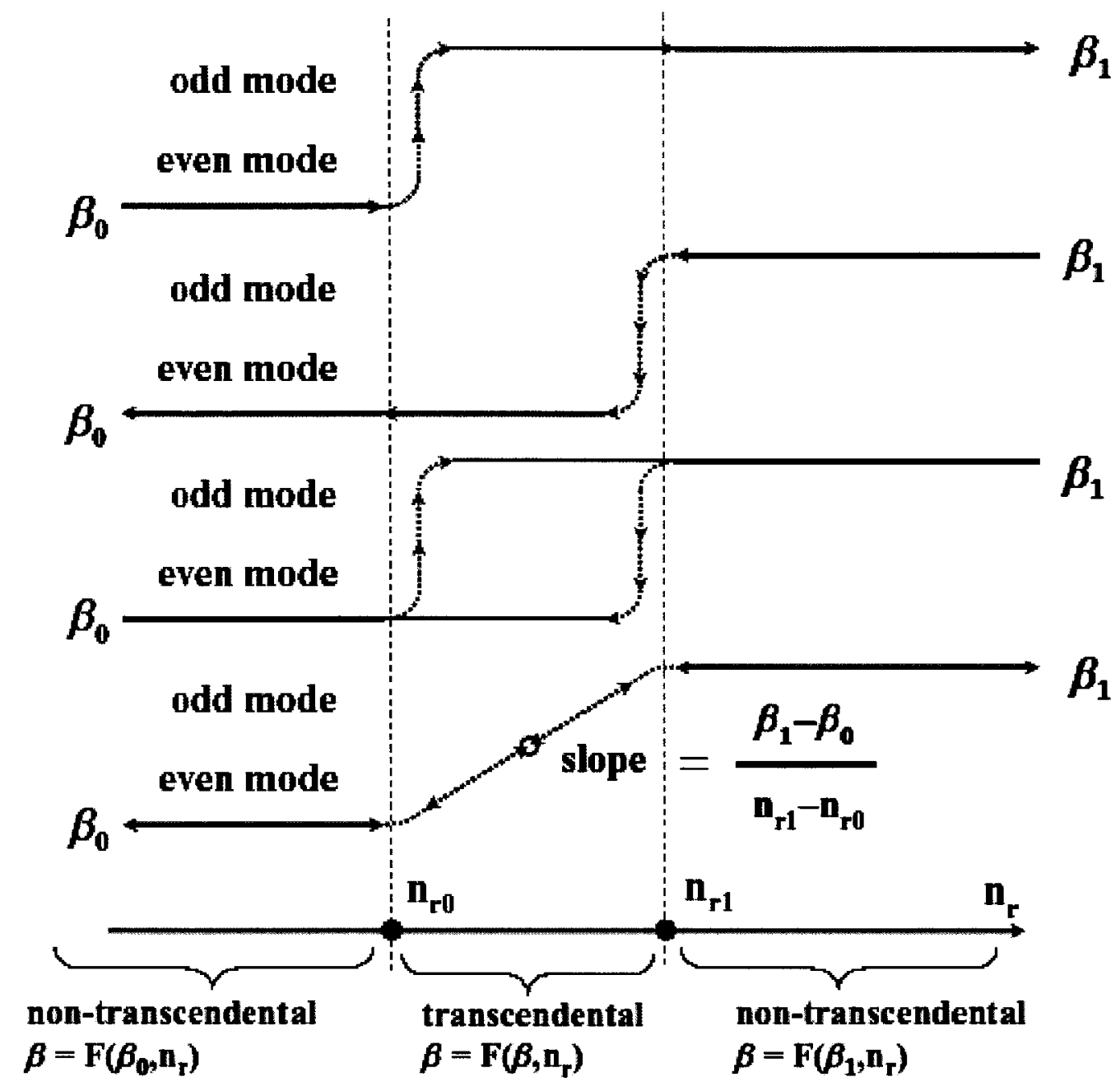

(a)

(b)

(c)

Fig. 3-10. Propagation constant, $\beta$ versus core refractive index, $n_{r}$.

(a) perturbative calculation when $n_{r}$ increases. Before $n_{r 0}$ solution is correct.

(b) perturbative calculation when $n_{r}$ decreases. After $n_{r 1}$ solution is correct.

(c) case (a) and (b) combined give "hysteresis" loop, from which an approximate slope can be found.

(d) proper approximate solution. 
Considering odd mode and $\phi_{3}=0$, then (3-69b) becomes,

$$
\begin{gathered}
\operatorname{coth}\left[u_{b} \mathrm{~s}\right]=-\frac{u_{r}}{u_{b}} \tan \left(\phi_{4}\right) \\
\operatorname{coth}\left[u_{b} s\right]=\frac{\frac{u_{r}}{u_{b}} \tan \left(u_{r} \mathrm{w}\right)-1}{\frac{u_{b}}{u_{r}} \tan \left(u_{r} \mathrm{w}\right)+1}
\end{gathered}
$$

Conditions (3-108), (3-110) involves solving transcendental equations. Which can be done numerically. Alternatively, we can use Taylor series expansion for $\tan \left(u_{r} \mathrm{w}\right)$ and solve it analytically. (4-th order algebraic equation is solvable in terms of radicals, its general solutions are presented in Appendix E ).

Equation (3-105) is quite a complicated transcendental equations for $n_{\text {eff }}$, to solve it analytically is a highly non-trivial task. Therefore, we will try to obtain an approximate analytical solution. In [38] a perturbation technique based on iterative procedure was applied to obtain approximate analytical solution of transcendental eigenvalue equation.

We will not use perturbation theory (PT) directly. The reason is that we know at this stage more information than PT assumes. Namely, we know the perturbed wavefunction, $\psi\left(n_{r 1}\right)$ $\left(e_{y}\left(n_{r 1}\right)\right)$, whereas PT takes unperturbed wavefunction, $\psi\left(n_{r 0}\right)\left(e_{y}\left(n_{r 0}\right)\right)$. And second, we know already characteristic equation (eigenvalue equation). So, applying PT directly would be like taking one step back. However, we will use the result obtained by PT (Lemma) and main assumption of PT (assumption: small perturbation does not affect propagation constant significantly).

We will use an iteration procedure to solve characteristic equation (eigenvalue equation). The characteristic equation for a five-layer slab is:

$\beta=F\left(\beta, n_{r}, P\right)$ 
where $F=k G, G$ is defined as in (3-105), $n_{r}$ is refractive index of the second channel, $P$ is the set of other parameters (widths, separation, indices of other layers) and $\beta$ is a propagation constant. To find $\beta$ we have to find the roots of equation $\beta-F\left(\beta, n_{r}, P\right)=0$

We know that $F$ is a non-decreasing function of $n_{r}$. When $n_{r}=0$, then five-layer slab is degenerated in a three-layer slab whose propagation constant $\beta_{0}$ is known. Characteristic equation for this case is:

$\beta_{0}=F\left(\beta_{0}, n_{r}=0, P\right)$

Now we introduce a small perturbation, $n_{r} \neq 0$ such that it does not significantly modifies modes of the waveguide and propagation constant. Then new propagation constant can be approximately calculated from the equation,

$\beta \cong F\left(\beta_{0}, n_{r}, P\right)$

which can be viewed as the first order iteration (or first order perturbation)

$\boldsymbol{\beta}_{1} \cong F\left(\boldsymbol{\beta}_{0}, n_{r}, P\right)$

$\boldsymbol{\beta}\left(n_{r}\right) \cong \boldsymbol{\beta}(0)+\Delta \boldsymbol{\beta}$

Results from (3-114) are valid for all values of $n_{r}$ that guarantee small deviation from unperturbed system, i.e. $n_{r}: \Delta \beta \ll \beta(0)$. When this condition start to break, the first order perturbation is not good any more. Then for such values of $n_{r}$ we can try higher orders:

$n_{r}: \Delta \beta \lll \beta(0)$

Second order

$\beta_{2} \cong F\left(\beta_{1}, n_{r}, P\right)=F\left(F\left(\beta_{0}, n_{r}, P\right), n_{r}, P\right)$

Third order

$\beta_{3} \cong F\left(\beta_{2}, n_{r}, P\right)=F\left(F\left(\beta_{1}, n_{r}, P\right), n_{r}, P\right)=F\left(F\left(F\left(\beta_{0}, n_{r}, P\right), n_{r}, P\right), n_{r}, P\right)$ 
n-th order

$$
\beta_{n} \cong F\left(\beta_{n-1}, n_{r}, P\right)=F\left(F\left(\ldots F\left(\beta_{0}, n_{r}, P\right), \ldots, n_{r}, P\right), n_{r}, P\right)
$$

When $n$ is large, then equation (3-120) is close to the original transcendental equation:

$$
\begin{gathered}
n \gg 1 \Rightarrow n-1 \approx n \Rightarrow \beta_{n-1} \approx \beta_{n} \\
\beta_{n} \cong F\left(\beta_{n-1}, n_{r}, P\right) \Rightarrow \beta_{n} \cong F\left(\beta_{n}, n_{r}, P\right)
\end{gathered}
$$

\subsection{Summary}

In this chapter we have derived a characteristic (eigenmode) equation for non-symmetric five-layer slab valid for TE mode starting from Maxwell's equations. Since that equation is transcendental it is impossible to extract a close form solution. We are searching for an alternative approximate solution in order to be able to optimize the device performance by quickly exploring different parameter sets. A close look reveals that there are certain regions in the computational domain (i.e. over the core refractive index $n_{r}$ ) where modal indices vary relatively 'slowly'. This suggests that we can use an iterative procedure and therefore turn the original transcendental equation in an ordinary algebraic equation with the right hand side containing modal indices from a previous iteration and left hand side containing the modal indices of current iteration. In the extreme conditions, when the derivative $\frac{d n_{e f f}}{d n_{r}}<<1$, we do not really need to update modal indices on the right hand side of the equation. Under such conditions finding approximate solution, $n_{e f f}\left(n_{r}\right)$ become a straightforward task. 


\section{Chapter 4}

\section{Results}

This chapter focuses on long period gratings written in a five-layer slab as shown in Fig. 4-1. We obtain various characteristics including the phase matching curves and transmission spectra of our waveguides and the required physical length for maximum switching. In section 4.1 we find an analytical expression for approximating the modal indices for fivelayer slab. Alternatively, we find modal indices numerically with software package called $\mathrm{C} 2 \mathrm{~V}$ (mode solver in $\mathrm{C} 2 \mathrm{~V}$ is based on one of the following calculation methods: transfer matrix method, Cauchy Integral method, method of Usievich or method of Bakhtazad). We then compare both results. A closed form analytical expression for modal indices is useful when we need an optimization of modal indices (or a function of them ) with respect to various parameters. This is the case if we search for the optimal parameters for a long period grating, since it is a function of modal indices, $\Lambda=\frac{\lambda}{n_{e f f}^{(n)}-n_{e f f}^{(m)}}$. An optimization can be done numerically. However, if the function has two or more arguments (in our case geometrical parameters and material refractive indices of the layers) the knowledge of the gradient of the function analytically can greatly speed up the optimization procedure.

The LPG in a five-layer slab, whose modal indices are already known, is examined in section 4.2. Under certain conditions the phase matching curves of the LPG exhibit a dual peak resonance, first observed in the fiber for higher order cladding modes, [39]. We discuss those specific conditions. We also obtain transmission spectra and a physical length for optimum devices. It turns out that the length is too large for any practical purposes. In section 4.3 we summarize results and outline possible approaches to reduce the total length of the device. 

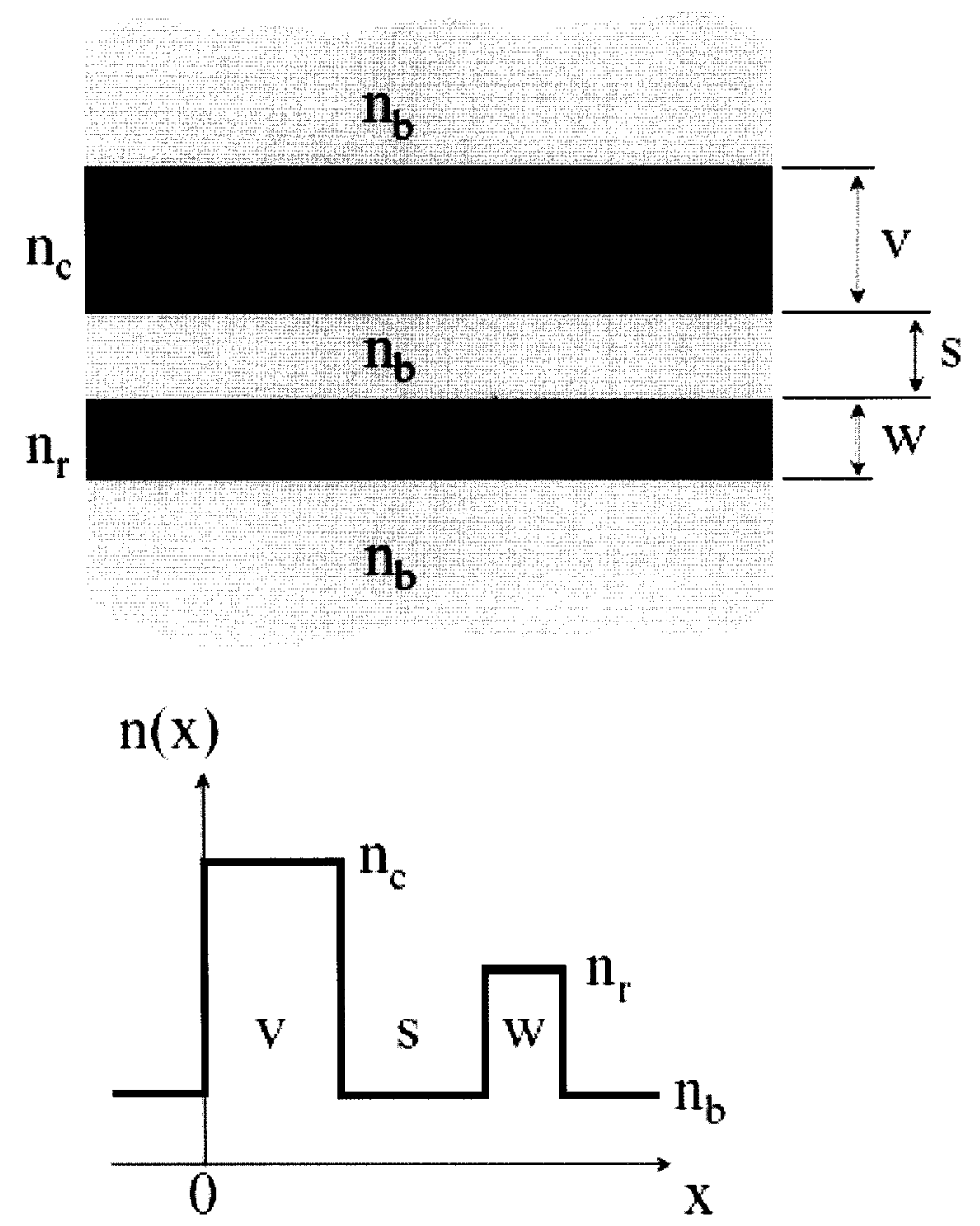

Fig. 4-1. Refractive index profile in a five-layer slab. 


\subsection{Approximate analytical modal indices for five-layer slab}

At this point we are ready to apply the analysis of the previous chapter to the actual problem. An illustration is presented in Fig. 3-10. Assume initial conditions (geometrical parameters and core refractive index $n_{r}$ ) such that the mode is even. To calculate the propagation constant we use the non-transcendental (perturbative) equation,

$\beta=F\left(\beta_{0}, n_{r}, P\right)$

This equation is valid up to a 'turning' point, i.e. up to the value of $n_{r 0}$ which satisfies conditions (3-108). After this, strictly speaking, we have to use the transcendental (nonperturbative) equation,

$\beta=F\left(\beta, n_{r}, P\right)$

however we do not use it, because we do not know how to solve it analytically. We continue to apply (4-1) even after $n_{r 0}$. The propagation constant $\beta$ does an abrupt but continuous transition, finally it arrives at a new steady level. The value for the new steady level for $\beta$ is correct, even though we did not use the proper equation (4-2). What is not correct is exactly when $\beta$ arrives there, i.e. the value $n_{r 1}$, the second 'turning' point, is not correct. To see why the new steady level is correct we can look at equations (3-65), (3-101).

$$
\begin{aligned}
& u_{c} \mathrm{v}=\phi_{L B}+\phi_{R B}+m \pi \\
& \tan \left(\phi_{R B}\right)=-\frac{u_{b}}{u_{c}} \operatorname{coth}\left[\phi_{3}\right] \\
& \Rightarrow u_{c} \mathrm{v}=\phi_{L B}+\arctan \left(-\frac{u_{b}}{u_{c}} \operatorname{coth}\left[\phi_{3}\left(n_{r}\right)\right]\right)+m \pi
\end{aligned}
$$

$u_{c} \mathrm{v}$ depends on $\arctan \left(f\left(n_{r}\right)\right)$, while the half phase shift at the left boundary, $\phi_{L B}$ does not depend on $n_{r}$. The function $\arctan (x)$ 'saturates' when its argument is large enough, i.e. at $\phi_{3}\left(n_{r}\right)$ near zero. This suggests that as a result of one single transition, the $u_{c} \mathrm{~V}$ acquires increment of about $\pi,(\arctan (\infty)-\arctan (-\infty)=\pi)$, no matter if the argument $\phi_{R B}$ changes a little bit faster or a little bit slower. (When the perturbation approach is valid then $\phi_{R B}$ is 
function of $n_{r}$ only. When the perturbation approach is not valid then $\phi_{R B}$ is function of $n_{r}$ and $\beta$, and this time the variation is due to $n_{r}$ and due to $\beta$ ). Now the question is how to find the correct second 'turning' point $n_{r 1}$. We start with value $n_{r}$ well above $n_{r 0}$ and decrease $n_{r}$ (negative perturbation). In the graph (Fig. 3-10b) it is seen as moving backwards in the $n_{r}$-axis. We also supply in the RHS of the equation (4-1) the propagation constant $\beta_{1}$ corresponding to the steady level calculated previously. Now the condition for the perturbative approach is satisfied since $\beta_{1}$ is steady and therefore the reaction of the system is small. This implies that the non-transcendental equation is applicable,

$\beta=F\left(\beta_{1}, n_{r}, P\right)$

The propagation constant slowly decreases towards the 'turning' point $n_{r 1}$, then it does a transition and reaches a steady state level, which is almost equal to $\beta_{0}$. Again, the exact value of $n_{r}$ when $\beta$ becomes $\approx \beta_{0}$ is not correct. However, we have calculated that value already. Now we can construct approximately the proper path of the actual transition, which otherwise would require solving the transcendental equation (4-2). The propagation constant $\beta$ before $n_{r}=n_{r 0}$ is known from (4-1), after $n_{r 1}$ it is known from (4-3). In between, according to the lemma (see Appendix A), $\beta$ must be a non-decreasing (monotonic) function. In the first order approximation, we assume it has constant slope, slope $=\frac{\beta_{1}-\beta_{0}}{n_{r 1}-n_{r 0}}$.

We have estimated analytically the behavior of $\beta$ over the range of $n_{r}$ (including the transition range) without solving the transcendental equation. This is illustrated in Fig. 3-10d. Observation. In the range $n_{b}<n_{r}<n_{r 0}$ the perturbation potential $\left(k^{2} n_{r}^{2}\right)$ may rise from 0 to $\sim 30 \%$ of the unperturbed potential, yet we are using over the whole range an old value of $\beta$ obtained from the three-layer slab. The calculation is reasonably accurate because the main assumption is valid: the perturbed system varies little from the unperturbed one (i.e. small reaction). In the range after $n_{r 0}$ the perturbed system starts to vary significantly, and the 
main assumption breaks down, the perturbative approach is not valid. This suggests that the true criteria which defines when a perturbation calculation is valid and when it is not valid is the 'reaction' of the system rather than the 'height' of the perturbation potential $\left(k^{2} n_{r}^{2}\right)$.

One more observation. The effect of the transcendental equation from $n_{r 0}$ to $n_{r 1}$ is the same as if feedback is introduced: what is obtained as a result, $\beta$ (the output), is substituted as an input in $F$. Solution with the feedback, $\beta=F\left(\beta, n_{r}, P\right)$ is slower than otherwise $\left(\beta=F\left(\beta_{0}, n_{r}, P\right)\right)$. For example, compare the sharp transition near $n_{r 0}$ in the graph from Fig. 3-10a and the slow gradual transition in the graph from Fig. 3-10d. Therefore the feedback performed by the function $F\left(\beta, n_{r}, P\right)$ is a negative feedback.

\section{Cut-off condition}

For the cut-off condition we have $n_{e f f}=n_{b}, \beta=k n_{b}$ and the previously defined quantities $u_{b}$, $u_{c}$ and $u_{r}$ now become $U_{b}, U_{c}$ and $U_{r}$ respectively:

$u_{c}^{2}=k^{2} n_{c}^{2}-\beta^{2}=k^{2}\left(n_{c}^{2}-n_{b}^{2}\right)=U_{c}^{2}$

$u_{r}^{2}=k^{2} n_{r}^{2}-\beta^{2}=k^{2}\left(n_{r}^{2}-n_{b}^{2}\right)=U_{r}^{2}$

$u_{b}^{2}=\beta^{2}-k^{2} n_{b}^{2}=k^{2}\left(n_{b}^{2}-n_{b}^{2}\right)=U_{b}^{2}=0$

We rewrite equation (3-68a) replacing $u_{b}$ and $u_{r}$ by $U_{b}$ and $U_{r}$ :

$U_{b} \tanh \left[U_{b} s+\phi_{3}\right]=-U_{r} \tan \left(\phi_{4}\right)$

$U_{b}=0 \Rightarrow \tan \left(\phi_{4}\right)=0$

The same result is valid for (3-68b). From (3-70) we have:

$$
\begin{aligned}
& U_{r} \tan \left(U_{r} \mathrm{w}+\phi_{4}\right)=U_{b}=0 \\
& \tan \left(U_{r} \mathrm{w}\right)+\tan \left(\phi_{4}\right)=0 \\
& \tan \left(U_{r} \mathrm{w}\right)=0 \\
& \Rightarrow U_{r} \mathrm{w}=m \pi, \quad m=0,1,2 \ldots
\end{aligned}
$$


$n_{\text {rcut-off }}=\sqrt{\left(\frac{m \pi}{k \mathrm{w}}\right)^{2}+n_{b}^{2}}$

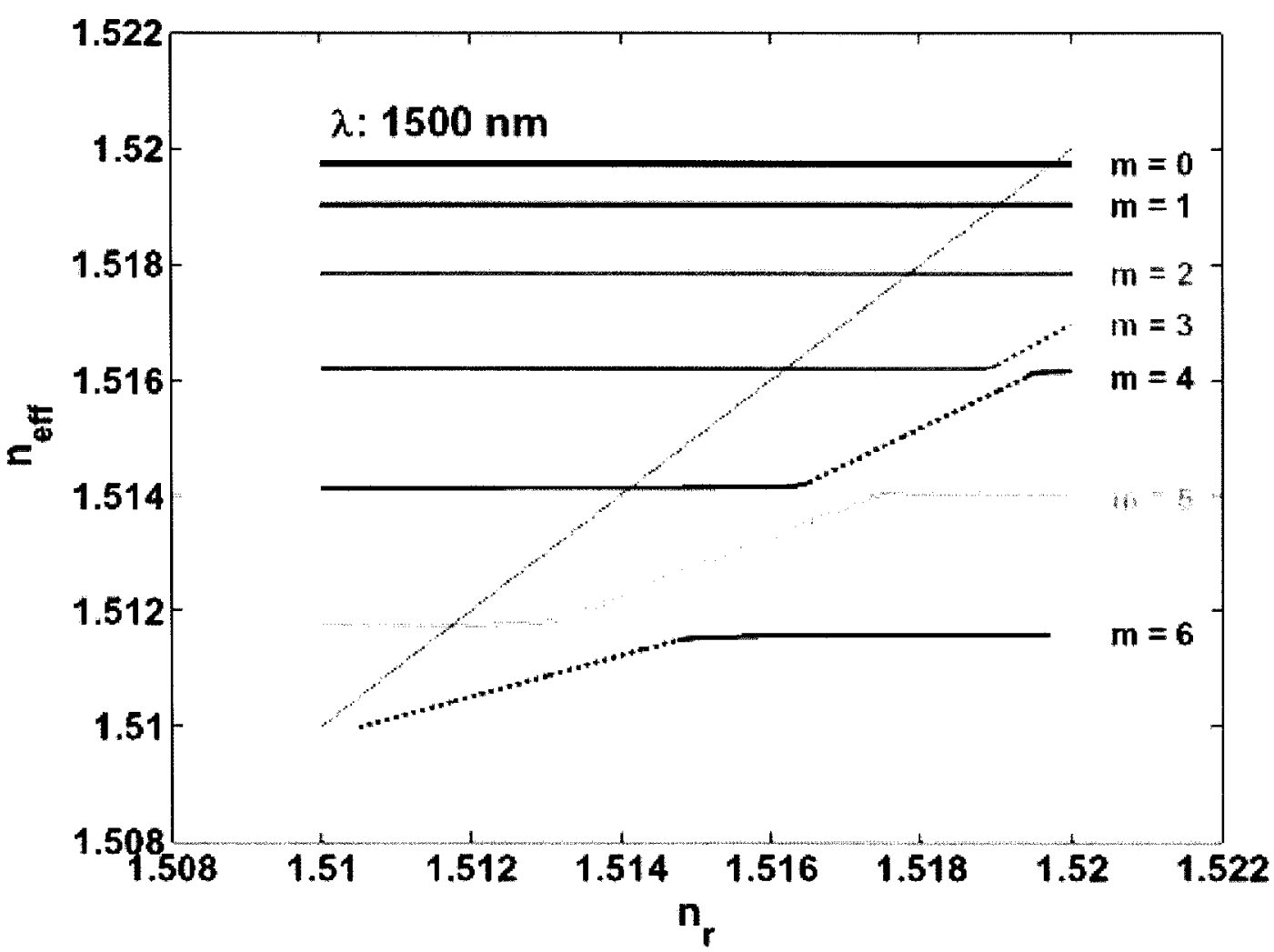

Fig. 4-2a. Approximate analytical solution. Modal indices versus core refractive index, $n_{r}$. Parameters: $\mathrm{v}=25 \mu \mathrm{m}, \mathrm{w}=5 \mu \mathrm{m}, \mathrm{s}=5 \mu \mathrm{m}, n_{\mathrm{c}}=1.52, n_{b}=1.51$, $\lambda=1500 \mathrm{~nm}$

Fig. 4-2a shows the modal indices as functions of $n_{r}$ obtained for an example with parameters $\mathrm{v}=25 \mu m, \mathrm{~s}=5 \mu \mathrm{m}, \mathrm{w}=5 \mu m, n_{b}=1.51, n_{c}=1.52$. We are using here typical parameters 
for ion-exchanged channel waveguides in glass [57]. The straight line $n_{\text {eff }}=n_{r}$ divides the graph in two partitions: $n_{e f f}>n_{r}$ represents a fast wave $\left(v_{p_{-} \text {mode }}>v_{p}\right.$, case 1 of Fig. 3.4$)$ and $n_{e f f}<n_{r}$ represents a slow wave $\left(v_{p_{-} \text {mode }}<v_{p}\right.$, case 2 of Fig. 3.4). We define slow and fast wave regions as follows. Let us call the modal phase velocity quantity $v_{p_{-} \text {mode }}=\frac{\omega}{\beta}=\frac{\omega}{k n_{e f f}}$ and the material phase velocity $v_{p}=\frac{\omega}{k_{r}}=\frac{\omega}{k n_{r}}$.

When the modal index is greater than the refractive index of the second channel, $n_{e f f}>n_{r}$ the modal phase velocity is smaller than the phase velocity, $v_{p_{-} \text {mode }}<v_{p}$. For this reason we call it the slow wave region. Similar, when $n_{e f f}<n_{r}$ the modal phase velocity is larger than the phase velocity in the second channel, $v_{p_{-} \text {mode }}>v_{p}$. This is the fast wave region. It is shown in Fig. 4-2b. 


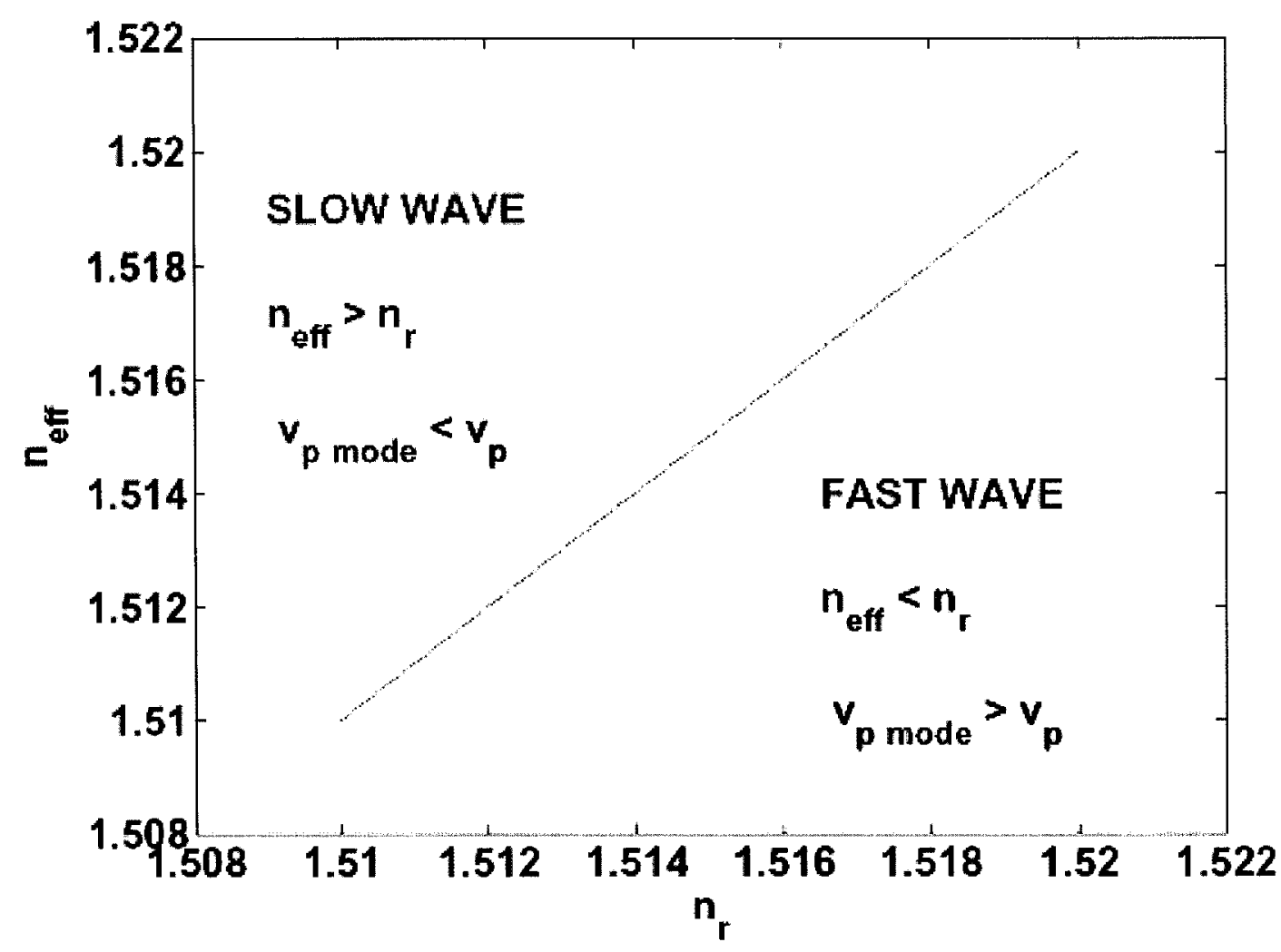

Fig. 4-2b. An illustration of slow and fast wave regions. When $n_{\text {eff }}>n_{r}$ the mode velocity is smaller than phase velocity in the second channel, $\mathrm{v}_{p_{-} \text {mode }}<\mathrm{v}_{p}$. This region we call slow wave region. When $n_{\text {eff }}<n_{r}$ the mode velocity is larger than phase velocity in the second channel, $\mathrm{v}_{p_{-} \text {mode }}>\mathrm{v}_{p}$. This region we call fast wave region.

\section{Physical picture, phase rotation}

From (3-65) we see that when the second channel is introduced and its refractive index starts to grow, it causes the half phase shift at the right side boundary, $\phi_{R B}$ (an equivalent to GoosHanschen phase shift) to rotate by $\pi$ during one transition (see Fig. 4-3). 


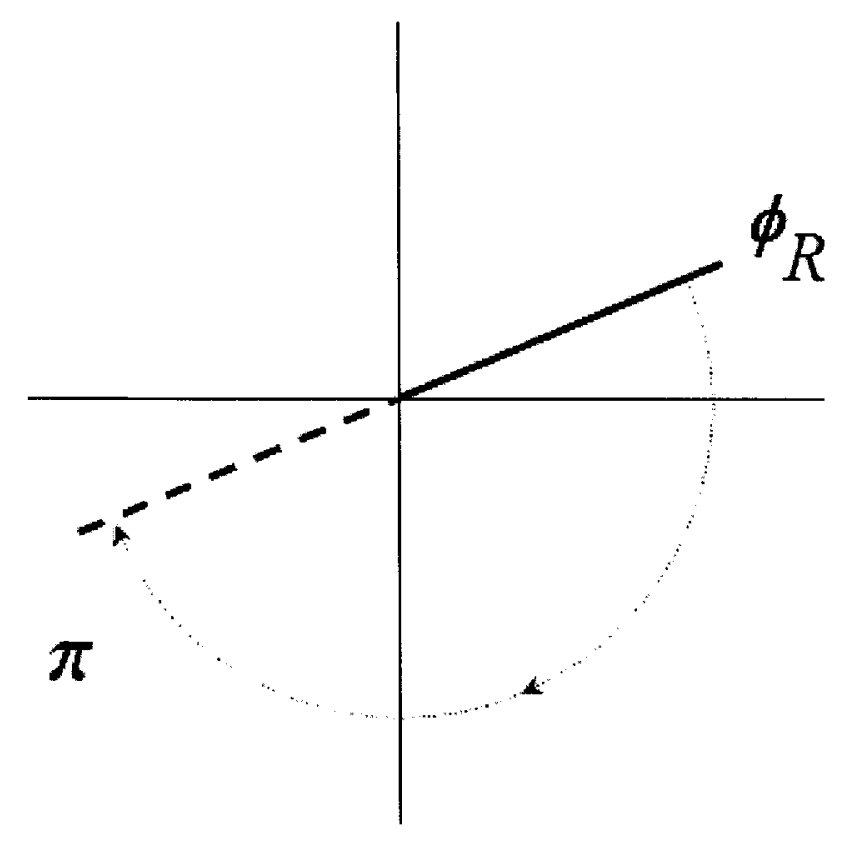

Fig. 4-3. Phase $\phi_{B R}$ rotation at the right side boundary of the wide channel during the transition.

The corresponding modal index rises up to the value equal to the next modal index. Since two distinct modes can not have equal modal index, whenever that condition is about to meet, the next modal index starts to make its transition, while the previous one saturates. Significant points for the calculation are those just before and just after the transition region for each mode. We have to evaluate them beforehand with (3-108), (3-110).

From the calculation point of view the value when $n_{e f f}=n_{r}$ has no special significance, there is no need to break the calculation domain in parts $n_{\text {eff }}>n_{r}$ and $n_{\text {eff }}<n_{r}$, therefore there is no need to find the value $n_{r}$ which divides two domains: $n_{r}=n_{\text {eff }}$. This saves a lot of calculations since $n_{r}=n_{\text {eff }}$ is a transcendental equation. 
What the perturbative approach fails to show properly is the appearance of new modes near cut-off. From (4-15) a new mode should start from $n_{\text {rcut-off }}=n_{b}=1.51$, for $m=0$ and $n_{\text {rcut }- \text { off }}=1.5174$ for $m=1$; corresponding to modal indices $n_{e f f}=n_{b}$. Calculation however shows that the new mode $m=0$ starts from $n_{\text {rcut-off }} \approx 1.51045$ (when $1.51<n_{r}<1.51045$ the modal index takes a value $n_{\text {eff }}<n_{b}$, which is nonsense ). Another new mode $m=1$ does not show up at all (its value again erroneous $n_{e f f}<n_{b}$ ). This is a limitation of the perturbative calculation.

An approximate analytical calculation for the modal indices can be useful whenever we have to optimize values $n_{e f f}$ (or its functions like $L P G=\frac{\lambda}{n_{e f f}^{(N)}-n_{e f f}^{(M)}}$ ) over certain parameters. An analytical expression allows one to evaluate the derivative analytically. This is more valuable than the numerical derivative especially when we search for $\mathrm{min} / \mathrm{max}$ over two or more parameters. (see [40]) Found this way, the optimal value can be used as a guess value for a further accurate numerical evaluation.

The approximate analytical solution agrees reasonably well with numerical results obtained by the commercially available software C2V. (see Fig. 4-4a, b) Fig. 4-4b presents a numerical simulation for our example obtained with $\mathrm{C} 2 \mathrm{~V}$. Starting values for modal indices calculated analytically are close to the ones calculated numerically. Upper modal indices ( $m=0,1,2,3,4)$ over the whole range of $n_{r}$ are close as well. Lower modal indices $(m=5,6)$ calculated numerically have relatively smooth transition regions compare to the sharp ones calculated analytically. Our assumption that the transition region can be approximated with a constant slope breaks down here. In fact this slope varies. 

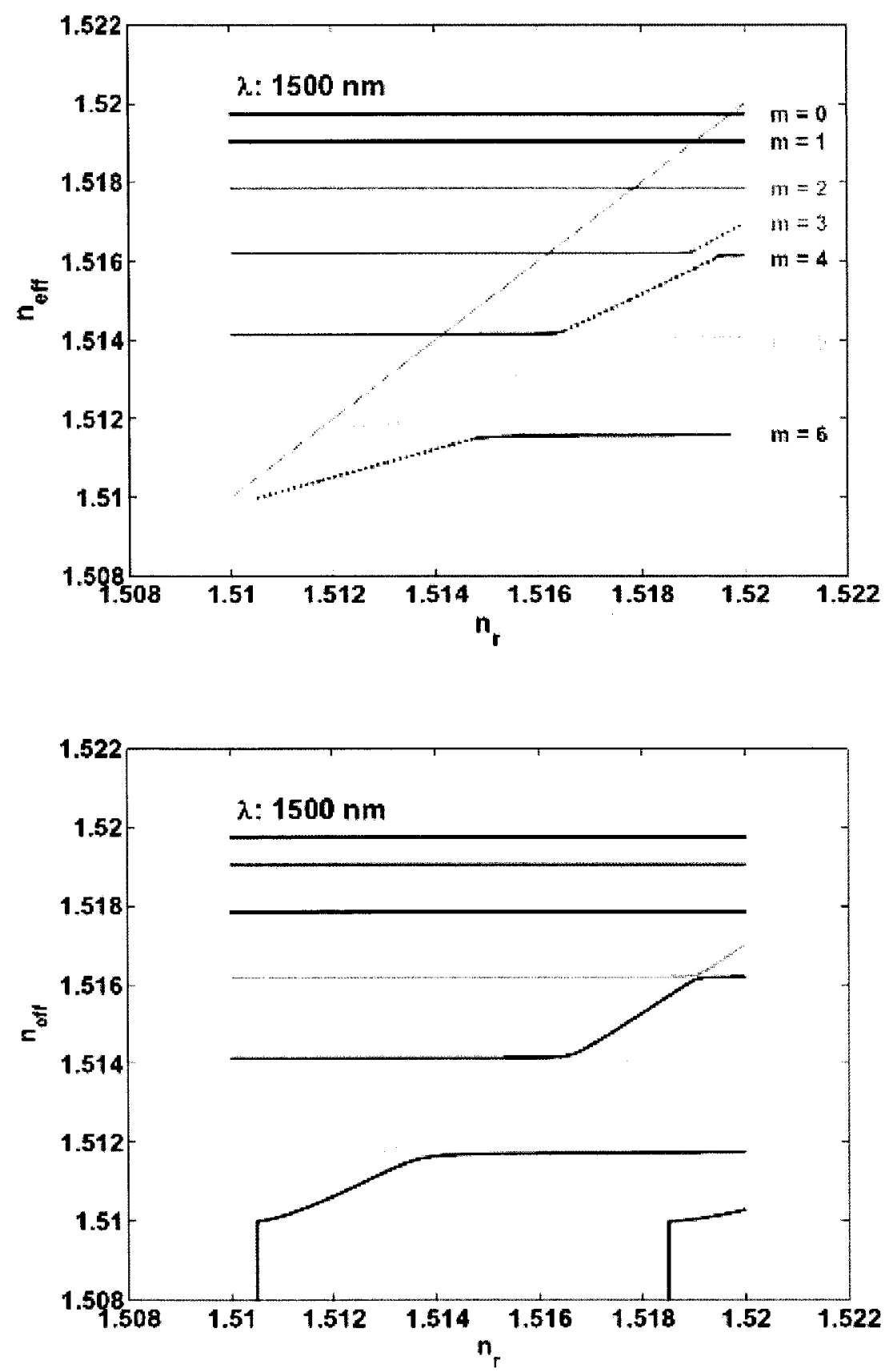

Fig. 4-4a, b. Modal indices versus core refractive index, $n_{r}$. Parameters: $\mathrm{v}=25 \mu \mathrm{m}$, $\mathrm{w}=5 \mu \mathrm{m}, \mathrm{s}=5 \mu \mathrm{m}, n_{c}=1.52, n_{b}=1.51, \lambda=1500 \mathrm{~nm}$.

(a) Top plot is approximate analytical solution.

(b) Bottom plot is numerical simulation obtained with software package C2V. 


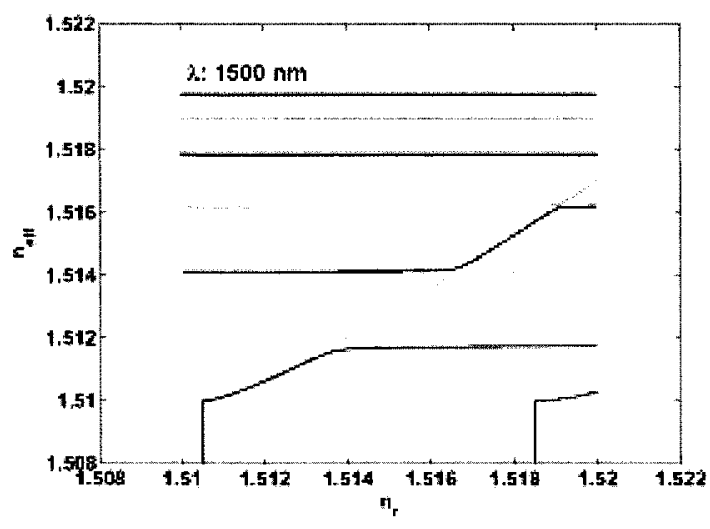

a)

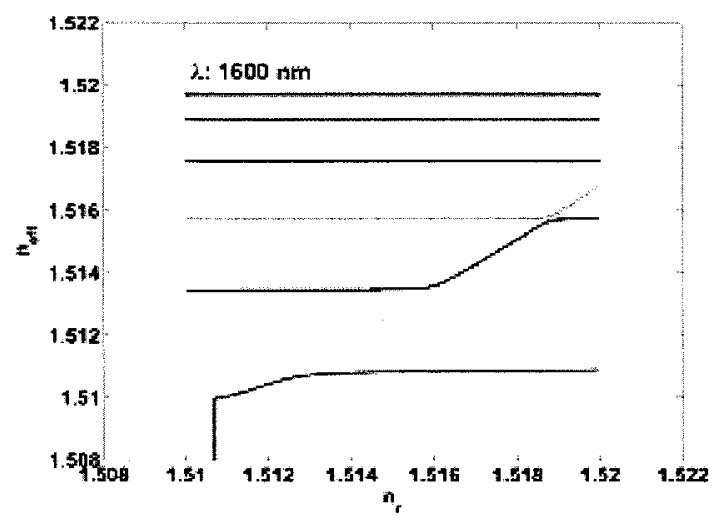

c)

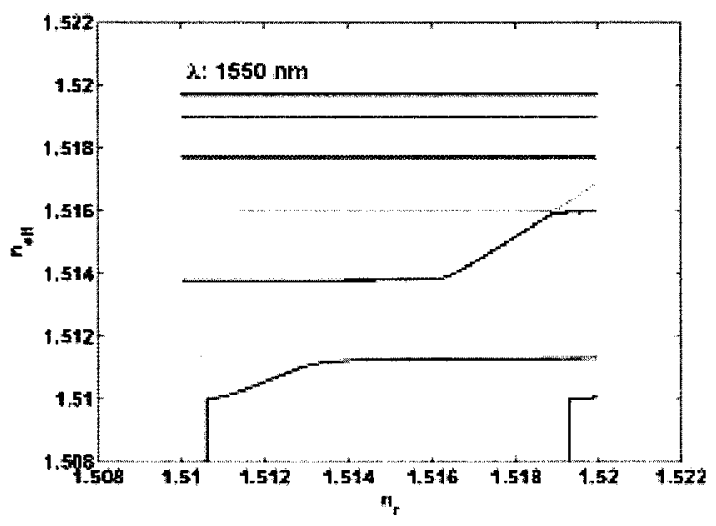

b)

Fig. 4-5. Modal indices versus core refractive index $n_{r}$ for three different wavelengths.

Parameters: $\mathrm{v}=25 \mu \mathrm{m}, \mathrm{w}=5 \mu \mathrm{m}, \mathrm{s}=5 \mu \mathrm{m}, n_{c}=1.52, n_{b}=1.51$
a) $\lambda=1500 \mathrm{~nm}$
b) $\lambda=1550 \mathrm{~nm}$
c) $\lambda=1600 \mathrm{~nm}$ 


\subsection{LPG in five-layer slab}

Modal effective indices, obtained with $\mathrm{C} 2 \mathrm{~V}$ for the example with the following parameters $\mathrm{v}=25 \mu \mathrm{m}, \mathrm{w}=5 \mu \mathrm{m}, \mathrm{s}=5 \mu \mathrm{m}, n_{c}=1.52, n_{b}=1.51, \lambda_{1}=1.5 \mu \mathrm{m}$ and $n_{r}$ going from $n_{b}$ to $n_{c}$ are shown in Fig. 4-5a. As we can see the modal indices do not spread uniformly over the range $n_{b}<n_{e f f}<n_{c}$, near the top $\left(n_{e f f} \sim n_{c}\right)$ the density of modes $\left(\frac{\Delta m}{\Delta n}\right.$, where $\Delta m$ is the number of modes, $\Delta n$ is the local range of indices) is higher and near the bottom $\left(n_{\text {eff }} \sim n_{b}\right)$ the density of modes is lower. This observation suggests, that if the wavelength changes then the modal indices at the top will not change too much, while modal indices at the bottom will. We are interested in weak $\lambda$-dependence because we want the phase matching condition to be almost satisfied over wide range of wavelengths. This 'flat' wavelength dependence would guarantee a large separation between distinct peaks in dual peak resonance. As we have seen from the literature review in chapter 1, the separation between two resonances is one of the most sensitive parameters to any external environmental change. We believe this separation would be also sensitive to internal changes of the refractive index and therefore sensitive to small nonlinear effects. Fig. 4-5b, c show the runs with wavelengths, $\lambda_{2}=1.55 \mu \mathrm{m}, \lambda_{3}=1.6 \mu \mathrm{m}$, which confirms the weak $\lambda$-dependence for $n_{\text {eff }}$ near $n_{c}$. One such mode, $m=3$ also has a 'turning' point, $n_{r 0} \approx 1.519$ near which the first derivative varies substantially. In that simulation the material dispersion $(n=n(\lambda))$ for each layer was assumed equal to the dispersion of fused silica with an appropriate 'shift' accounting for the correct value of $n$.

It is instructive to visualize three dimensional plots of $\left(n_{\text {eff } 0}-n_{\text {eff } 3}\right)$ versus $n_{r}$ and $\lambda$. The surface $\left(n_{\text {eff } 0}-n_{\text {eff } 3}\right)$ has two parts, a 'plateau' with very a gentle slope along the $\lambda$-axis and a 'steep descent' representing the transition region (see Fig. 4-6a). The 'edge' between two surfaces is almost parallel to $\lambda$-axis but not quite. When the value of $n_{r}$ is chosen properly, then $\left(n_{\text {eff } 0}-n_{\text {eff } 3}\right)$ intersects the 'edge' somewhere in the middle of the wavelength range, i.e. at $\lambda_{0} \approx 1.55 \mu \mathrm{m}$. The path, i.e. surface section with constant $n_{r 0}$, starts at $\lambda_{\text {start }}=1.5 \mu \mathrm{m}$ on 
'steep descent', then intersects the 'edge' at around $\lambda_{0} \approx 1.55 \mu \mathrm{m}$ and finishes at $\lambda_{\text {stop }}=1.6 \mu \mathrm{m}$ on the 'plateau'. It is shown in Fig. 4-6b, $\mathrm{c}$ as the boundary between colors and two dashed lines show the path when the value $n_{r}$ is modulated, $n_{r}=n_{r 0} \pm \Delta$. If the 'edge' is sharp, then the $\left(n_{\text {eff } 0}-n_{\text {eff } 3}\right)$-path corresponding to operation at fixed $n_{r 0}$ lies partly on the 'steep descent' (half) and partly on the 'plateau' (second half). However, if the 'edge' is smooth, then we cannot say that the path lies partially on the 'steep descent' and partially on the 'plateau'. It lies entirely on the "edge". In this case $\left(n_{e f f 0}-n_{e f f}\right)$ is weakly depends on $\lambda$ over a wide range of wavelengths. 


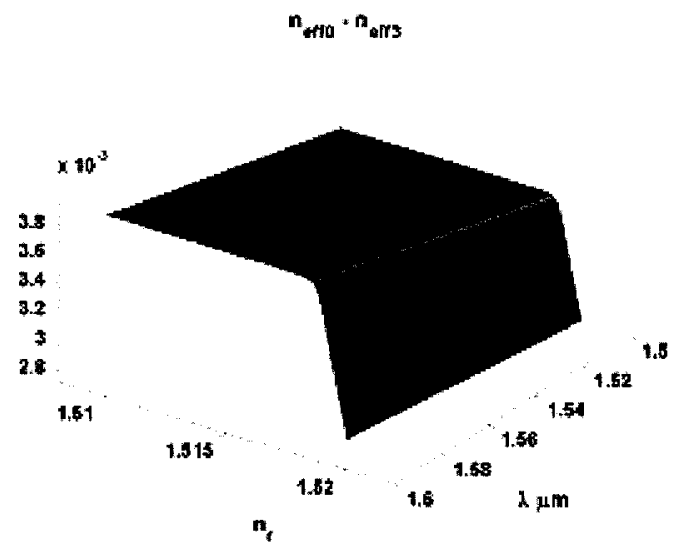

a)

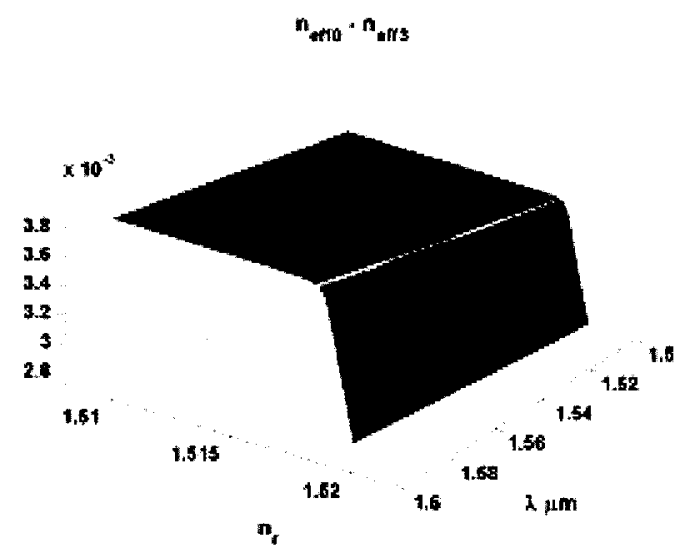

b)

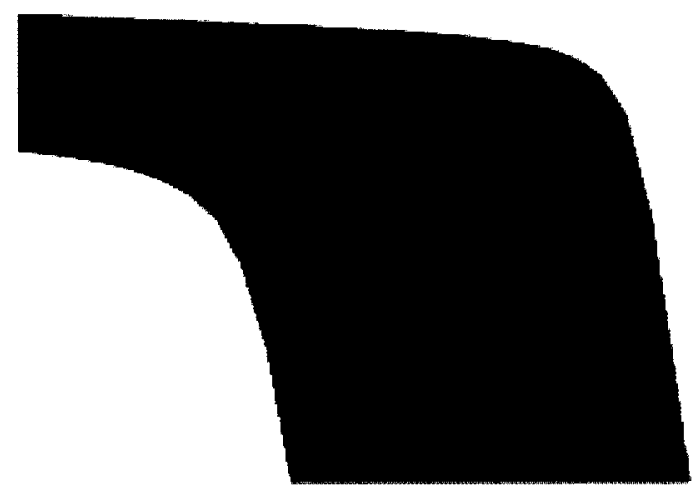

c)

Fig. 4-6. Surface plot $\left(n_{\text {eff } 0}-n_{\text {eff } 3}\right)$ versus core refractive index, $n_{r}$ and wavelength. Parameters: $\mathrm{v}=25 \mu \mathrm{m}, \mathrm{w}=5 \mu \mathrm{m}, \mathrm{s}=5 \mu \mathrm{m}, n_{c}=1.52, n_{b}=1.51$ a) $\left(n_{\text {eff } 0}-n_{\text {eff } 3}\right)$

b) boundary between two colors is an operation line with constant $n_{r} \approx 1.519$. Two dashed lines show path when value $n_{r}$ is modulated, $n_{r}=n_{r 0} \pm \Delta$.

c) Enlarged version of Fig. 4-6b. 
To reduce the $\lambda$-dependence further we have to reduce the gentle slope of the 'plateau' by moving to the lower mode, $\left(m=2,\left(n_{\text {eff } 0}-n_{\text {eff } 2}\right)\right)$ where the density of modes is higher and therefore the $\lambda$-dependence weaker. For comparison we have shown surface plots for a higher mode, $\left(n_{\text {eff } 0}-n_{\text {eff } 5}\right)$ versus $n_{r}$ and $\lambda$ in Fig. $4-7 \mathrm{a}, \mathrm{b}$. 


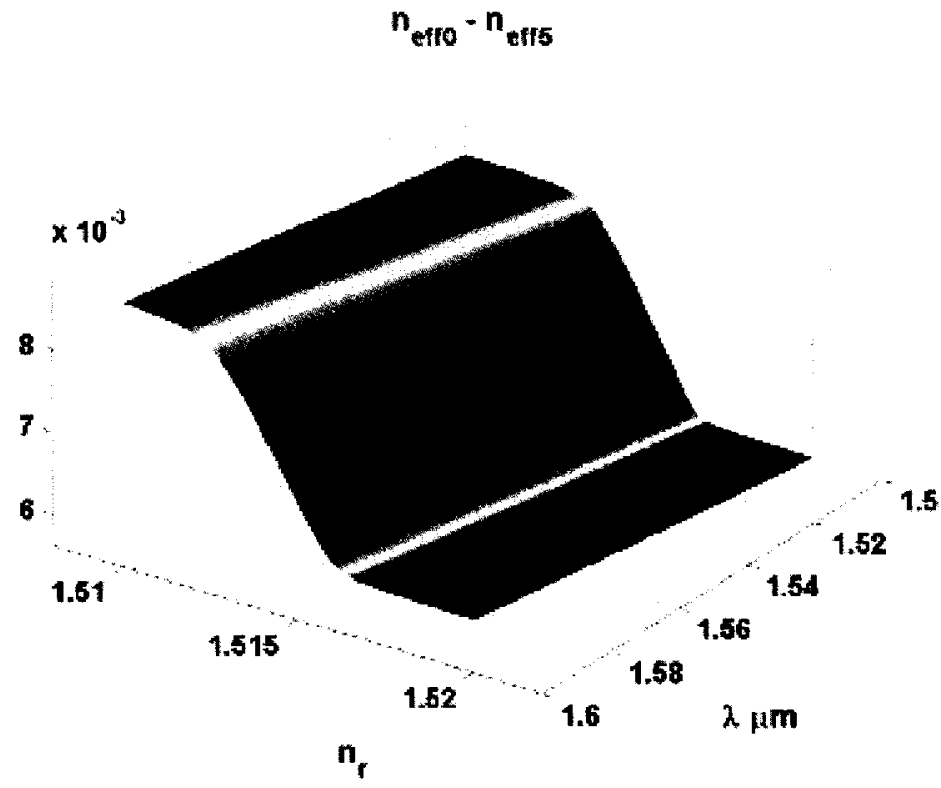

a)

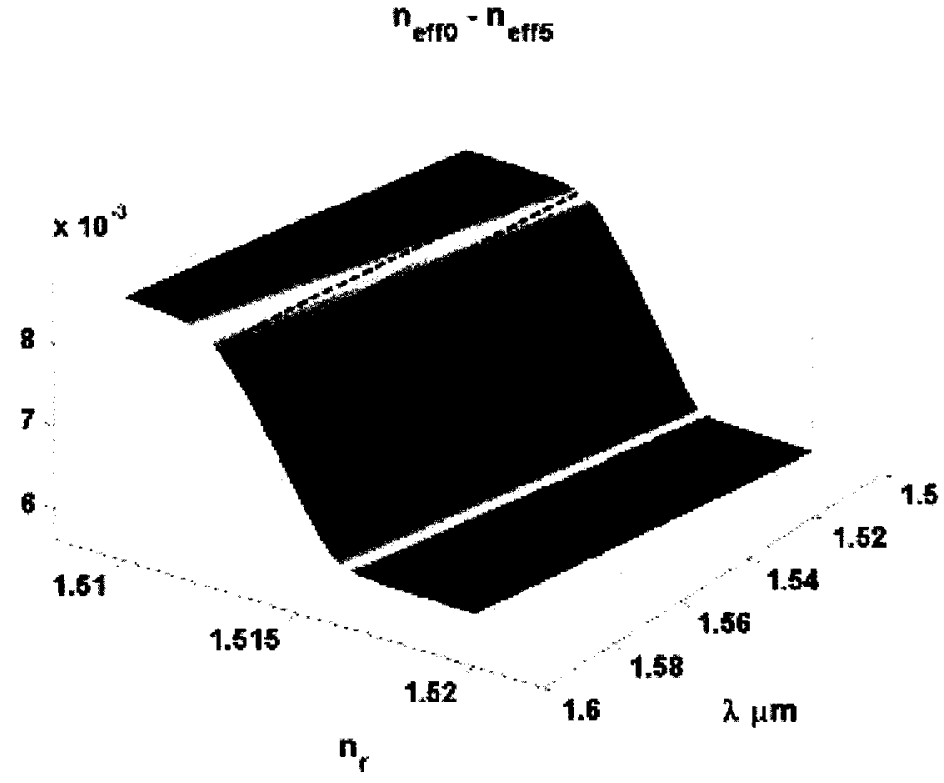

b)

Fig. 4-7. Surface plot $\left(n_{\text {eff } 0}-n_{\text {eff } 5}\right)$ versus core refractive index, $n_{r}$ and wavelength.

Parameters: $\mathrm{v}=25 \mu \mathrm{m}, \mathrm{w}=5 \mu \mathrm{m}, \mathrm{s}=5 \mu \mathrm{m}, n_{c}=1.52, n_{b}=1.51$

a) $\left(n_{\text {eff } 0}-n_{\text {eff } 5}\right)$ 
b) Boundary between two colors is an operation line with constant $n_{r} \approx 1.513$. Two dashed lines show path when value $n_{r}$ is modulated, $n_{r}=n_{r 0} \pm \Delta$.

The corresponding phase matching curves, $\mathrm{LPG}=\Lambda=\frac{\lambda}{n_{\text {eff } 0}-n_{\text {eff } 3}}$ (see chapter 1 for definition) constructed between modes $m=3$ and $m=0$ near $n_{r 0} \approx 1.519$ should have a relatively weak $\lambda$-dependence ('flat' along $\lambda$-axis).

LPG-curves for that example with $n_{r}=n_{r 0}-10^{-6}, n_{r}=n_{r 0}, n_{r}=n_{r 0}+10^{-6}$ are shown in Fig. 4-8a. If we choose a constant value for the LPG period, $\Lambda \approx 423.56 \mu \mathrm{m}$ then the middle curve will have exactly one resonance condition (pure phase matching condition:

$\beta_{0}-\beta_{3}=\frac{2 \pi}{\Lambda}$ ); the bottom curve has two intersections with $\Lambda \approx 423.56 \mu \mathrm{m}$, so it has two distinct wavelengths when the phase matching condition is met; and top curve has no resonance condition over the whole range of wavelengths. 


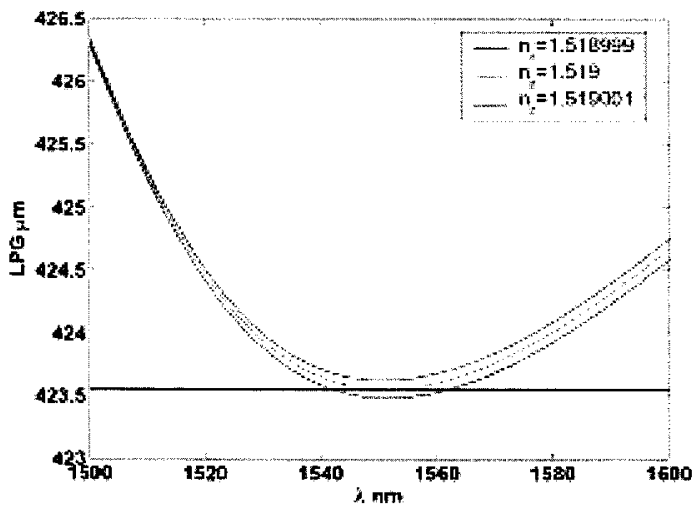

a)

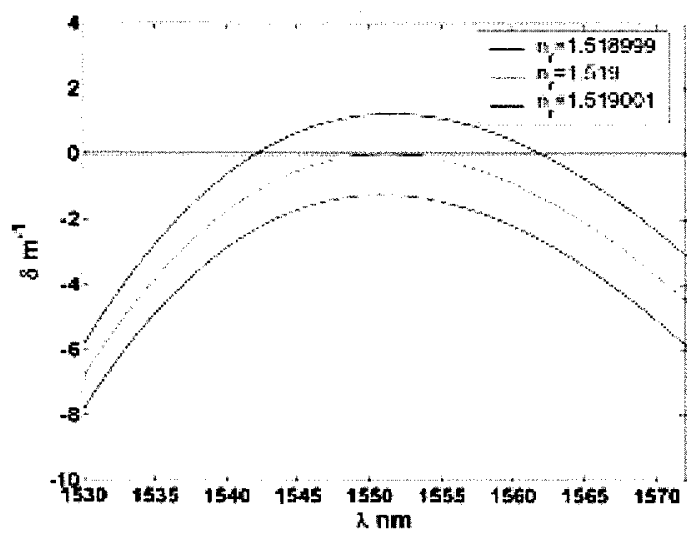

c)

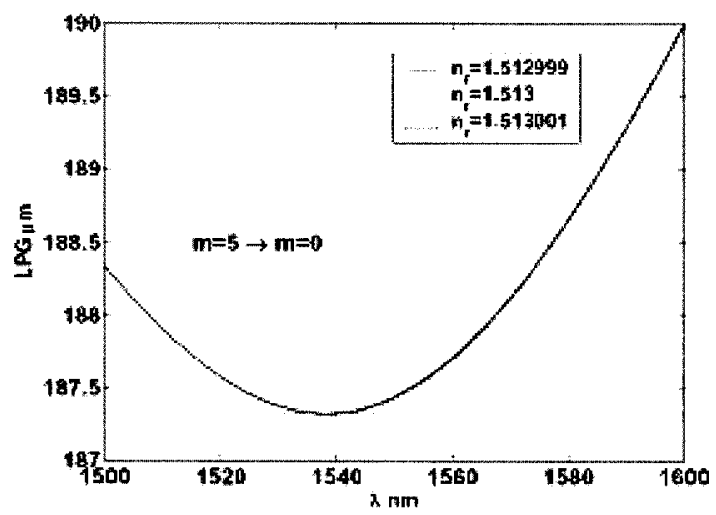

b)

Fig. 4-8. Plot phase matching curves versus wavelength for three values of core refractive index. Parameters: $\mathrm{v}=25 \mu \mathrm{m}, \mathrm{w}=5 \mu \mathrm{m}, \mathrm{s}=5 \mu \mathrm{m}, n_{\mathrm{c}}=1.52$, $n_{b}=1.51$.

a) $\Lambda=\frac{\lambda}{n_{\text {eff } 0}-n_{\text {eff } 3}}$, core refractive index: $n_{r}=1.519-10^{-6}, n_{r}=1.519$, $n_{r}=1.519+10^{-6}$. 
b) $\Lambda=\frac{\lambda}{n_{\text {eff } 0}-n_{\text {eff } 5}}$, core refractive index: $n_{r}=1.513-10^{-6}, n_{r}=1.513$, $n_{r}=1.513+10^{-6}$.

c) $\delta=\frac{1}{2}\left[\left(\beta_{0}-\beta_{3}\right)-\frac{2 \pi}{\Lambda}\right]$, core refractive index: $n_{r}=1.519-10^{-6}$, $n_{r}=1.519, n_{r}=1.519+10^{-6}$.

It is interesting now to present LPG-curves for coupling between $m=5$ and $m=0$ near the 'turning' point $n_{r} \approx 1.513$ (see Fig. $4-8 \mathrm{~b}$ ), the $n_{r}$-dependence is much weaker (all three curves almost overlap) in this case. Corresponding detuning curves for coupling between mode $m=3$ and mode $m=0$ ( $\delta$ curves) are shown in Fig. $4-8 \mathrm{c}$.

The transmission characteristic of that device should have one dip for the middle curve $\left(n_{r}=n_{r 0}\right)$, two distinct dips for the top curve $\left(n_{r}=n_{r 0}-10^{-6}\right)$ and no dip for the bottom curve $\left(n_{r}=n_{r 0}+10^{-6}\right)$. This in general is true, however we have to keep in mind that each resonance has a finite spectral width, and so does each dip in the transmission characteristic. If that width is larger than the spectral separation between resonances, then two distinct dips will overlap and appear as one dip.

To find out under what conditions this may happen we investigate the functional dependence of the transmission characteristic,

$$
T=1-\frac{\sin ^{2}\left(\kappa L_{g} \sqrt{1+\left(\frac{\delta}{\kappa}\right)^{2}}\right)}{1+\left(\frac{\delta}{\kappa}\right)^{2}}
$$

where

$\kappa$ is the coupling coefficient between mode $m=3$ and $m=0$ 
$\delta$ is the detuning from the resonance condition (the phase matching condition),

$\delta=\frac{1}{2}\left[\left(\beta_{0}-\beta_{3}\right)-\frac{2 \pi}{\Lambda}\right]$

$L_{g}$ is the total length of LPG. Assume that the total length is such that provides $100 \%$ coupling between the modes, then $\kappa L_{g}=\frac{\pi}{2}$. The graph of the function $y(x)=1-\frac{\sin ^{2}\left(0.5 \pi \sqrt{1+x^{2}}\right)}{1+x^{2}}$ is shown in Fig. 4-9. When $x=0$, the function takes zero value, $y(x=0)=1-\sin ^{2}(0.5 \pi)=0$. When $x= \pm \sqrt{3}$, it takes a maximum value, 1 : $y( \pm \sqrt{3})=1-\frac{\sin ^{2}(0.5 \pi \sqrt{1+3})}{1+3}=1-\frac{\sin ^{2}(\pi)}{4}=1-0=1$. Therefore to see effect of the modulation, $\kappa$ must not be too large compare to $\delta$, otherwise $\kappa>\delta, \frac{\delta}{\kappa}<<1, x<<1$ and $y<<1$ for all the spectral range, so the modulation of the transmission characteristic practically does not exist. The largest acceptable value of $\kappa$ should be such that $-\sqrt{3} \kappa\left(\lambda_{0}\right)$ be roughly equal to the maximum of the lower phase matching curve ( $\delta$ curve) and $+\sqrt{3} \kappa\left(\lambda_{0}\right)$ be roughly equal to the maximum of the upper phase matching curve (see Fig. 410). Then the modulation effect will be prominent: one dip in transmission characteristic, two distinct dips, none. Two distinct dips in this case are as close to each other as possible before getting overlapped. If the value of $\kappa$ is larger than this, then the two dips start to overlap and at $\lambda=\lambda_{0}$ the transmission characteristic with two dips will not be equal to 1 . If on the other hand the value of $\kappa$ is smaller, then the two dips will be further apart from each other. For our example the limiting value of $\kappa$ is $\frac{1.24}{\sqrt{3}} \approx 0.716 \mathrm{~m}^{-1}$ and the LPG length therefore is $L_{g}=\frac{\pi}{2 \kappa}=\frac{\pi}{2 \times 0.716} \approx 2.194 \mathrm{~m}$. 


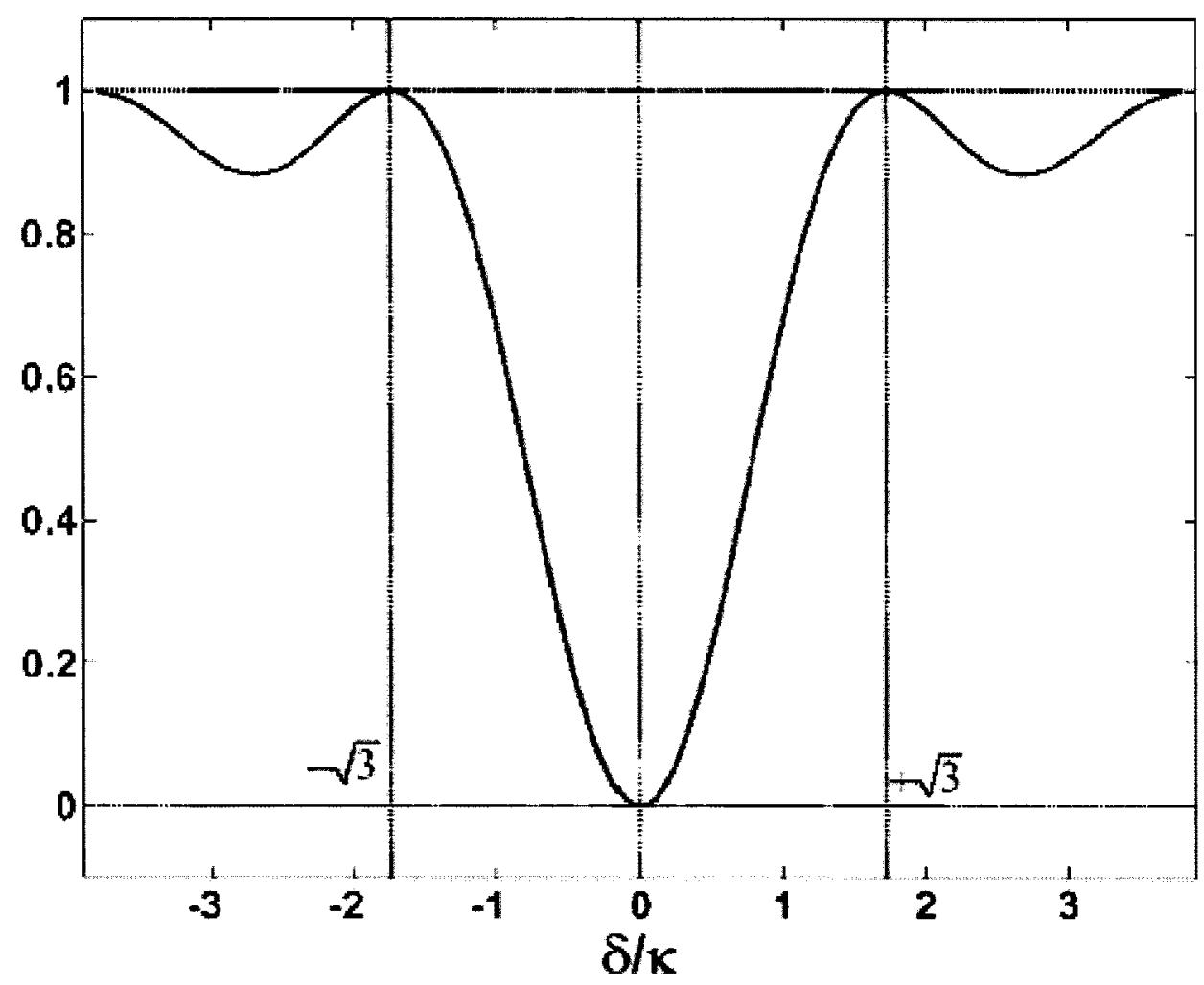

Fig. 4-9. Plot power transmission characteristic for LPG element versus $\left(\frac{\delta}{\kappa}\right)$ $y(x)=T=1-\frac{\sin ^{2}\left(0.5 \pi \sqrt{1+x^{2}}\right)}{1+x^{2}}, x=\frac{\delta}{\kappa}$. Where $\delta$ is detuning from phase matching condition, $\kappa$ is coupling coefficient. 


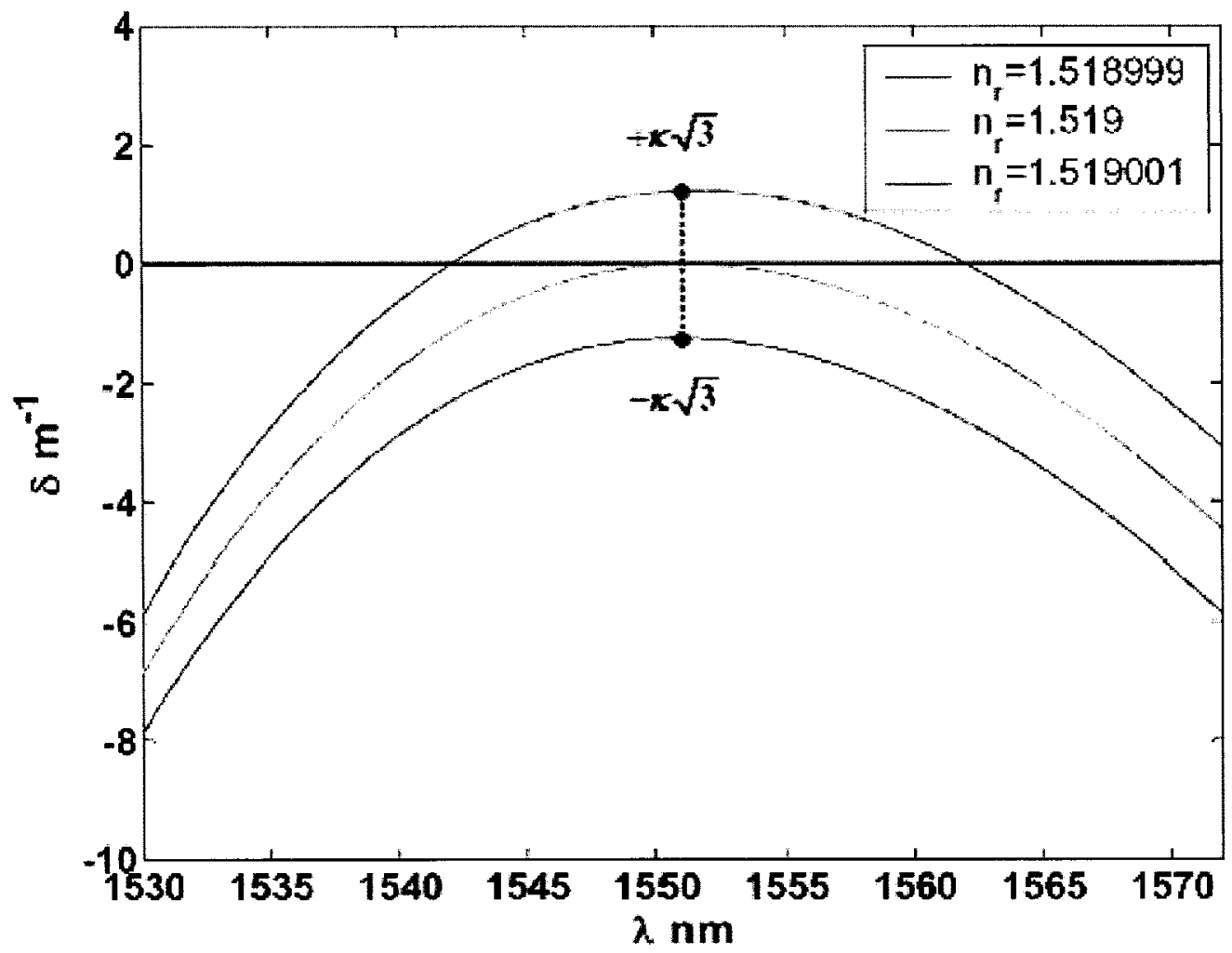

Fig. 4-10. Maximum values of $\delta$ for top curve should be about $+\kappa\left(\lambda_{0}\right) \cdot \sqrt{3}$ and for bottom curve about $-\kappa\left(\lambda_{0}\right) \cdot \sqrt{3}$ for transmission characteristic modulation from 0 to 1

The transmission characteristics for $n_{r}=n_{r 0}-10^{-6}, n_{r}=n_{r 0}+10^{-6}$ and for several intermediate values of $n_{r}$ are presented in Fig. 4-11a-i. 


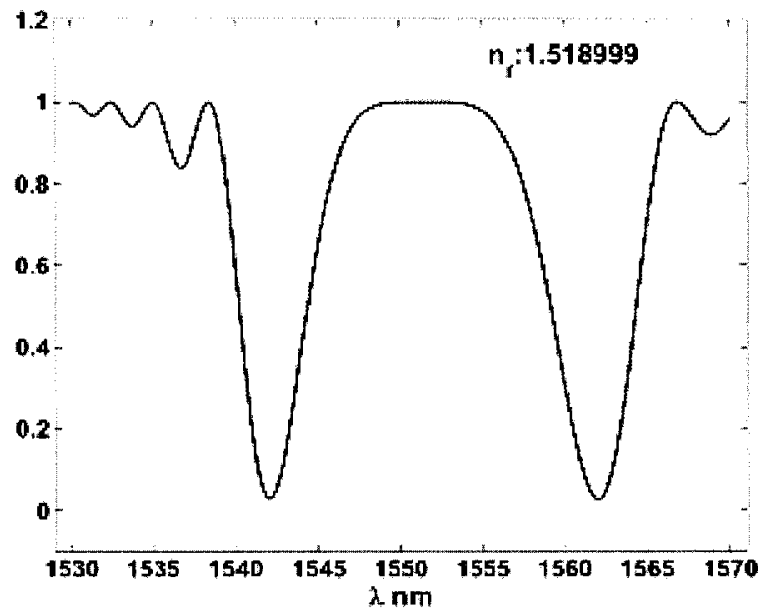

a)

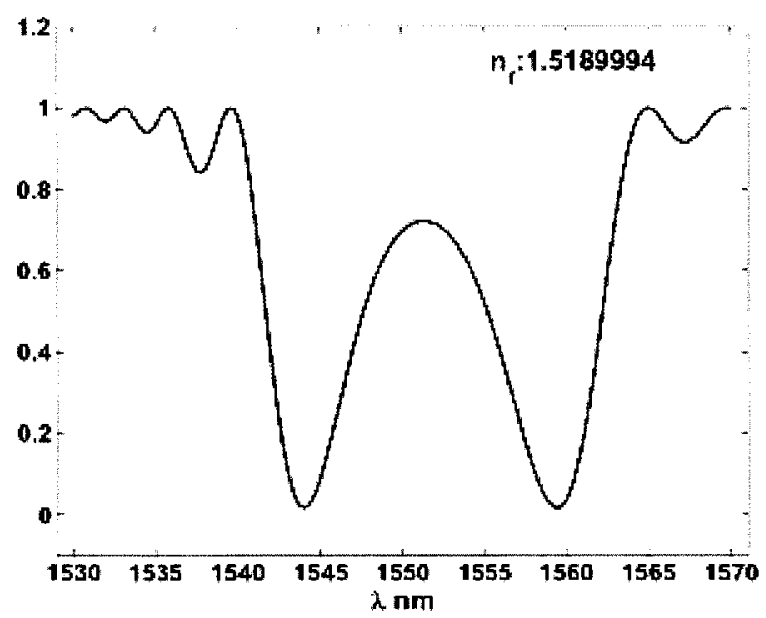

b)

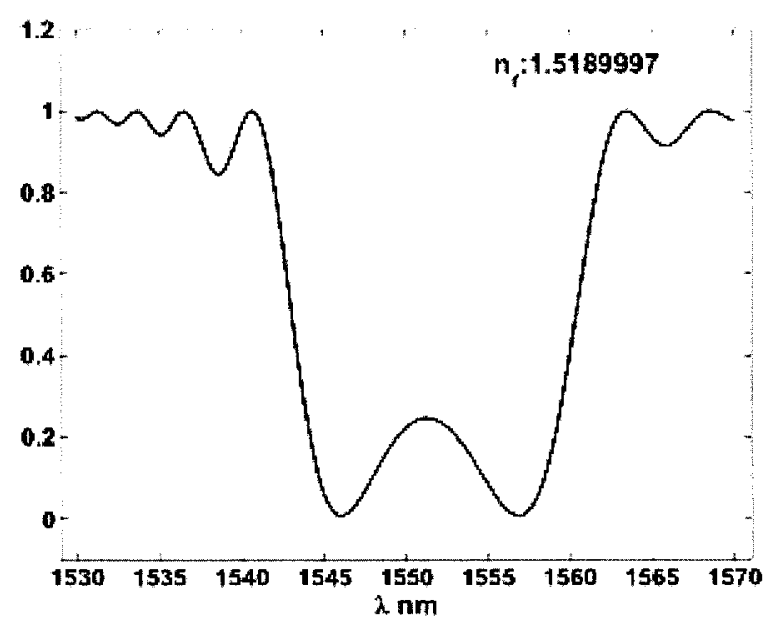

c) 


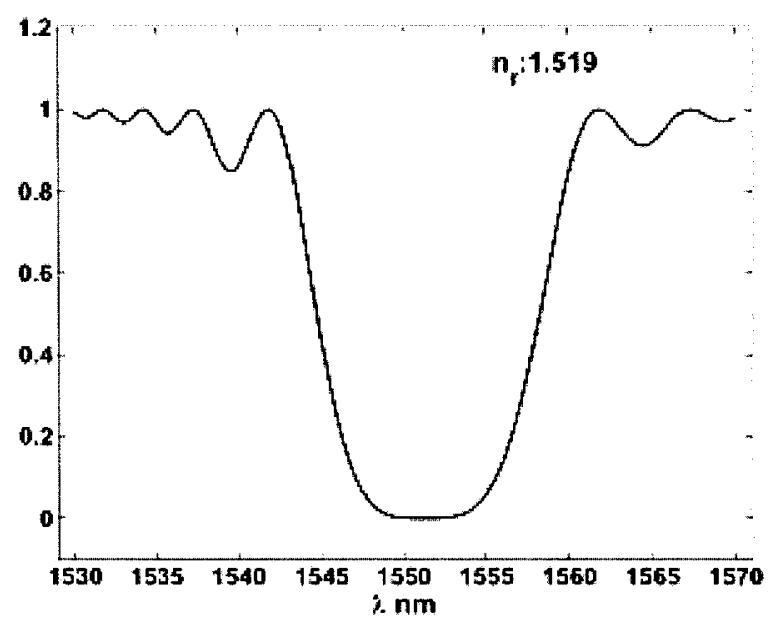

d)

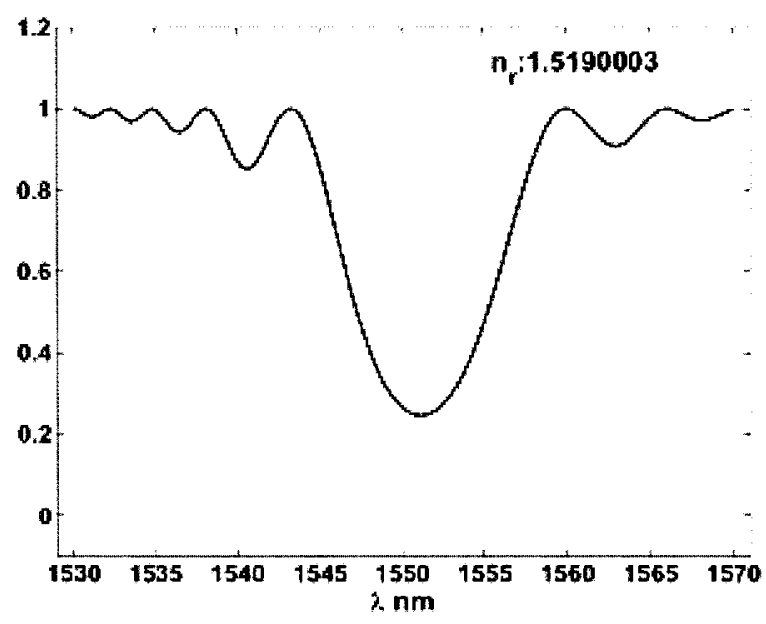

e)

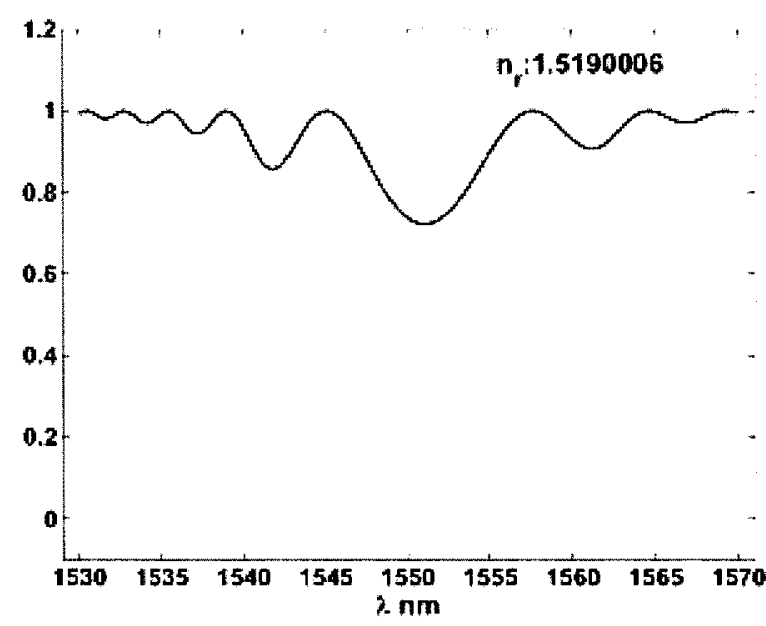

f) 


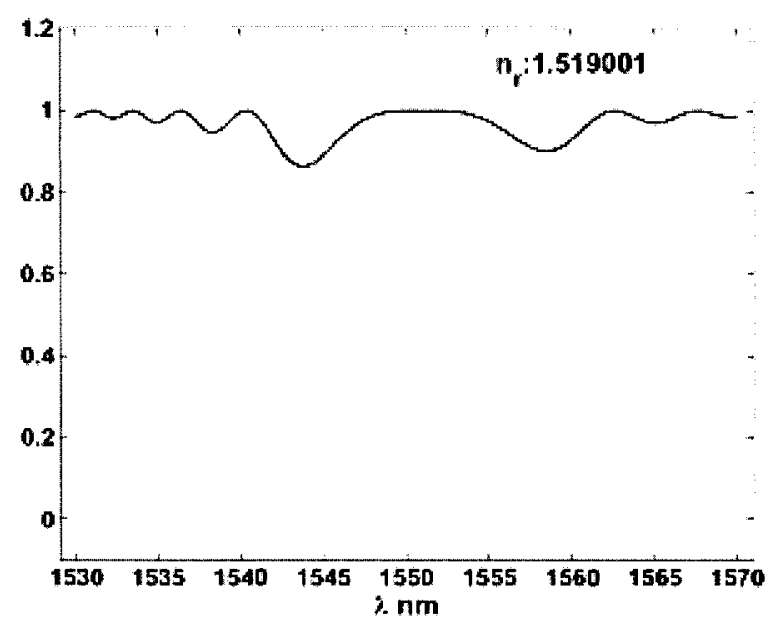

g)

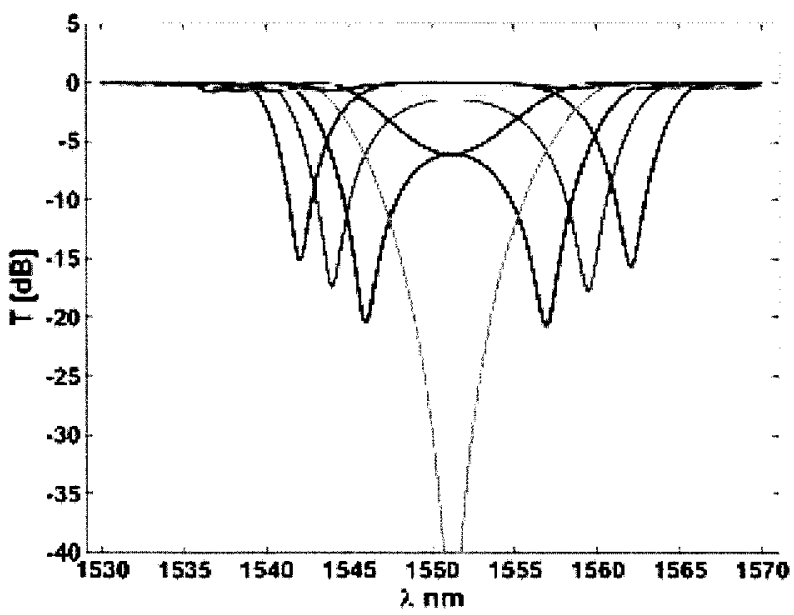

h)

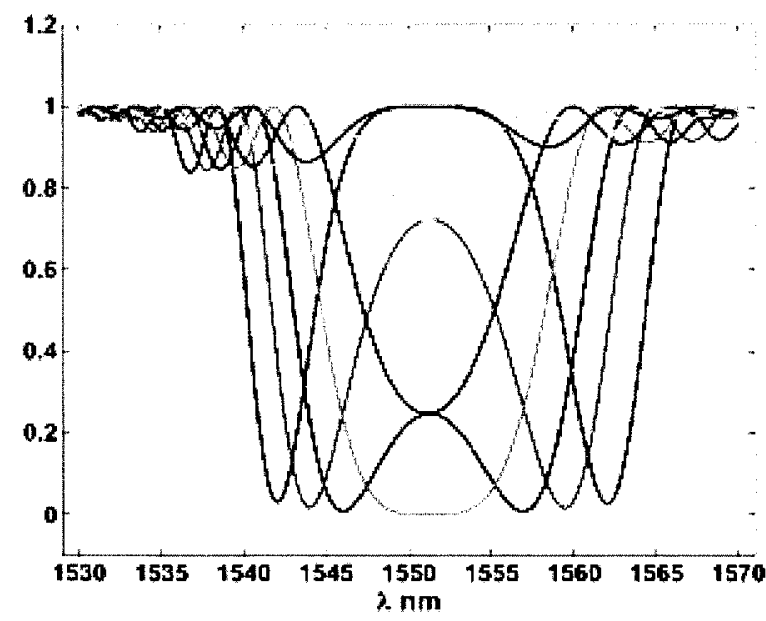

i) 
Fig. 4-11. Transmission characteristic of a five-layer slab with LPG for various core refractive indeces.
a) $n_{r}=1.5189990$
b) $n_{r}=1.5189994$
c) $n_{r}=1.5189997$
d) $n_{r}=1.5190000$
e) $n_{r}=1.5190003$
f) $n_{r}=1.5190006$
g) $n_{r}=1.5190010$
h) all in one plot
i) all in one plot, in units of $[\mathrm{dB}]$.

From these graphs we can estimate the sensitivity parameter $\frac{\Delta T}{\Delta n_{r}}[d B]$ for two cases, when the dips are distinct and they are 'moving sideways' (dotted line in Fig. 4-12a) and when only one dip is left and it is 'moving upwards' (dotted line in Fig. 4-12b). In the first case $\frac{\Delta T}{\Delta n_{r}} \approx 0.0000597\left[d B / 10^{-6}\right.$ u.r.i. $]$ at $\lambda \approx 1542 \mathrm{~nm}$ and in the second case
$\frac{\Delta T}{\Delta n_{r}} \approx 0.000062\left[d B / 10^{-6}\right.$ u.r.i. $]$ at $\lambda \approx 1551.2 \mathrm{~nm}$. 


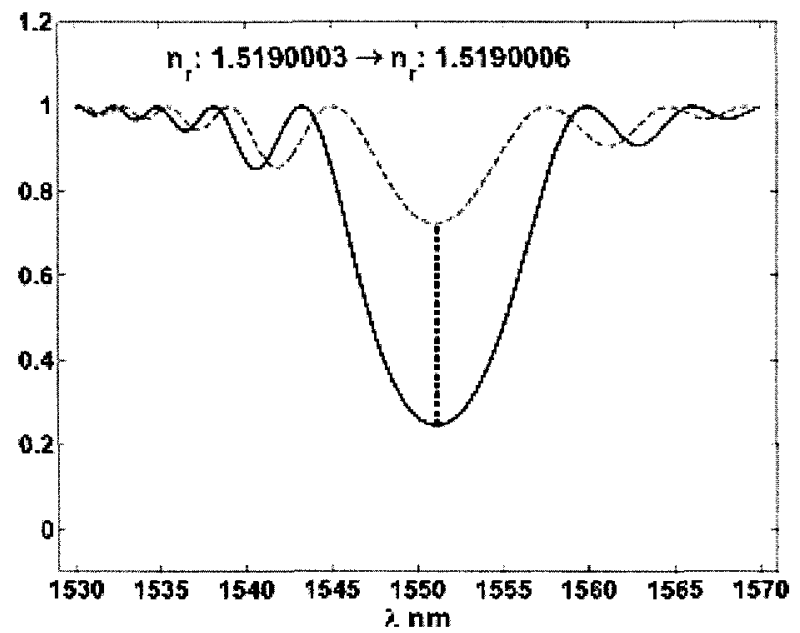

a)

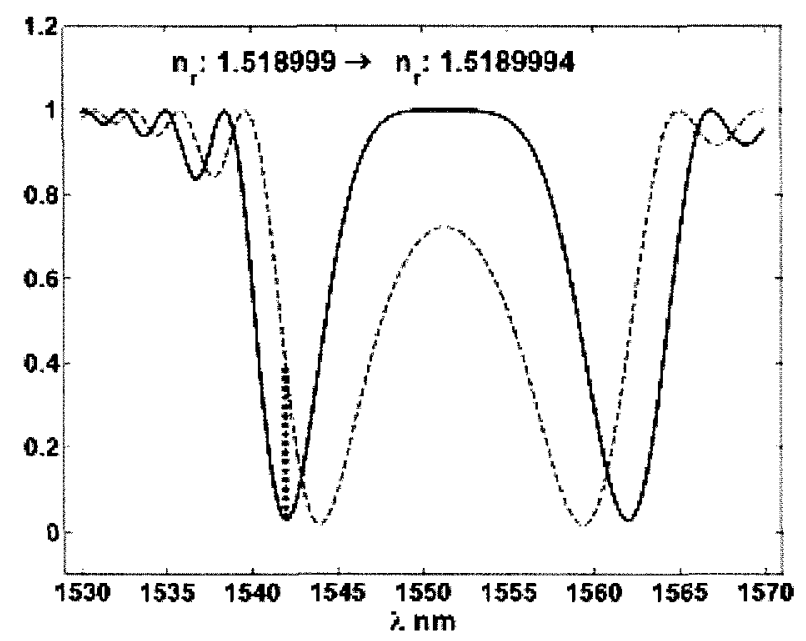

b)

Fig. 4-12. Transmission characteristics, $T(\lambda)$ of a five-layer slab with LPG. Core refractive index is modulated:

a) from $n_{r}=1.5189990$ to $n_{r}=1.5189994$. Transmission characteristic appears to move sideways. At wavelength $\lambda=1542 \mathrm{~nm} T$ varies by $\Delta T \approx 0.37$ and $\frac{\Delta T}{\Delta n_{r}} \approx 0.0000597\left[d B / 10^{-6}\right.$ u.r.i. $]$. 
b) from $n_{r}=1.5190003$ to $n_{r}=1.5190006$. Transmission characteristic appears to move upwards. At wavelength $\lambda=1551.2 \mathrm{~nm} T$ varies by $\Delta T \approx 0.48$ and $\frac{\Delta T}{\Delta n_{r}} \approx 0.000062\left[d B / 10^{-6}\right.$ u.r.i. $]$.

\subsubsection{Influence of geometry and material choice}

Now, we want to investigate how the geometrical parameters of the structure $(\mathrm{v}, \mathrm{s}, \mathrm{w})$ can modify the phase matching curves. First, we want to see if it is possible to 'push' the modal index with turning point closer to a lower order mode, i.e. towards $n_{c}$. If so, then we can expect to get phase matching conditions with even weaker $\lambda$-dependence and possibly with stronger $n_{r}$-dependence near the 'turning point'. 


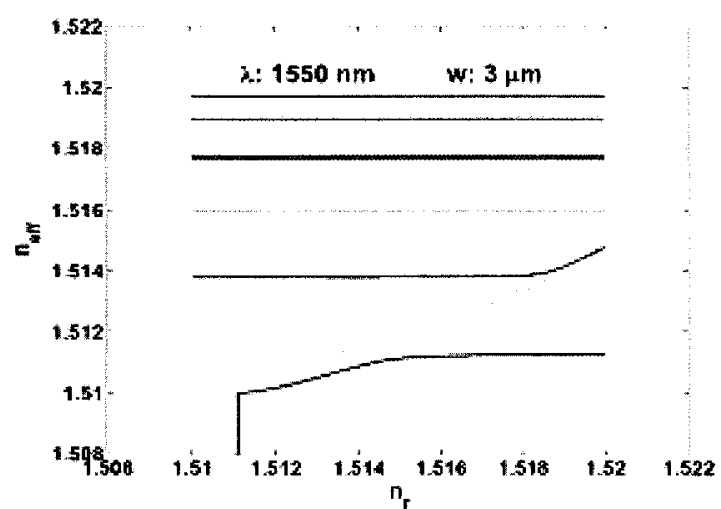

a)

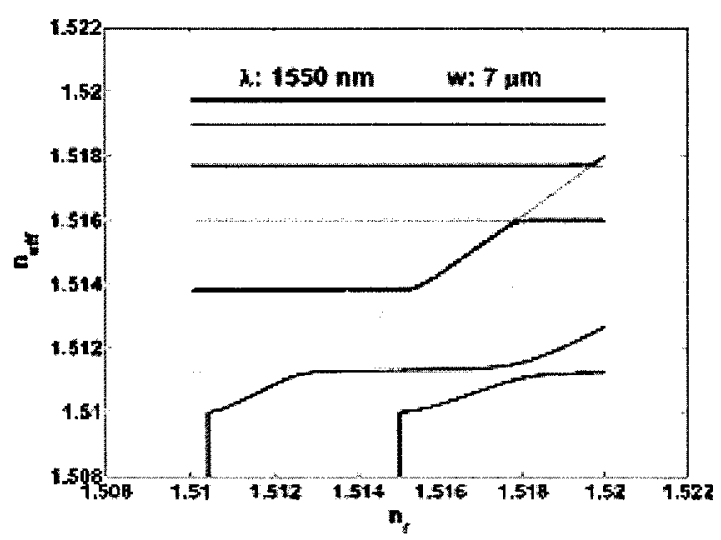

c)

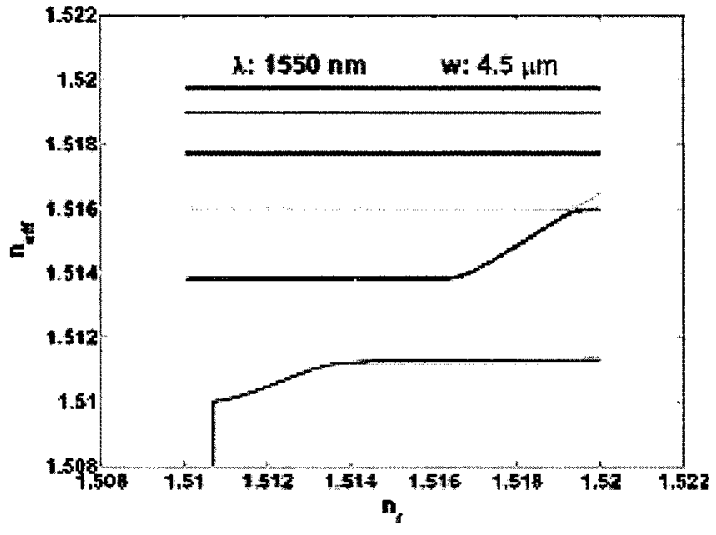

b)

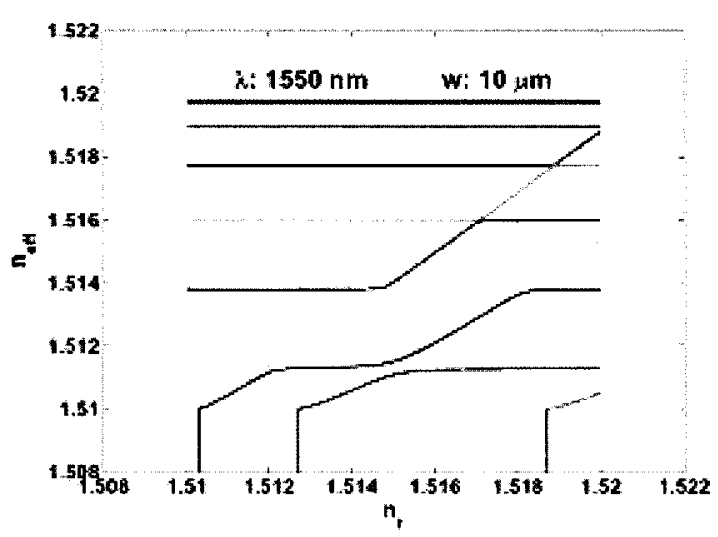

d)

Fig. 4-13. Modal indices versus core refractive index, $n_{r}$ for various widths of the core. Parameters: $\mathrm{v}=25 \mu \mathrm{m}, \mathrm{s}=5 \mu \mathrm{m}, n_{c}=1.52, n_{b}=1.51, \lambda=1550 \mathrm{~nm}$
a) $\mathrm{w}=3 \mu \mathrm{m}$
b) $\mathrm{w}=4.5 \mu \mathrm{m}$
c) $\mathrm{w}=7 \mu \mathrm{m}$
d) $\mathrm{w}=10 \mu \mathrm{m}$ 
Fig. 4-13a-d show modal indeces versus core refractive index for various widths, $w$ of the second channel (core) from $\mathrm{w}=3 \mu \mathrm{m}$ to $\mathrm{w}=10 \mu \mathrm{m}$. For a wider core the transition slope of modal indices gets larger, so it can reach the next higher mode as with $\mathrm{w} \geq 7 \mu \mathrm{m}$. When this happens the correspondent phase matching curves become 'flat' along the $\lambda$-axis (see Fig. 4-15a). The limiting value of $\kappa$ for that condition ( $\mathrm{w}=8 \mu \mathrm{m}, m=2 \rightarrow m=0$ ) is $\frac{1.76}{\sqrt{3}} \approx 1.016 \mathrm{~m}^{-1}$ and the $100 \%$ coupling length is $L_{g}=\frac{\pi}{2 \kappa}=\frac{\pi}{2 \times 1.016} \approx 1.546 \mathrm{~m}$. However, a wider core makes the second channel a multimode channel, which is undesirable. While launching light in a multimode channel, it is very difficult to control which mode exactly is excited, that is why we prefer to deal with a single mode channel. 


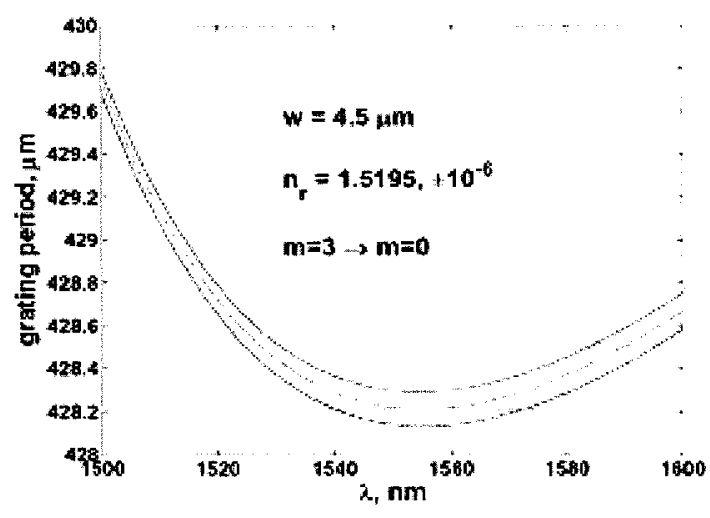

a)

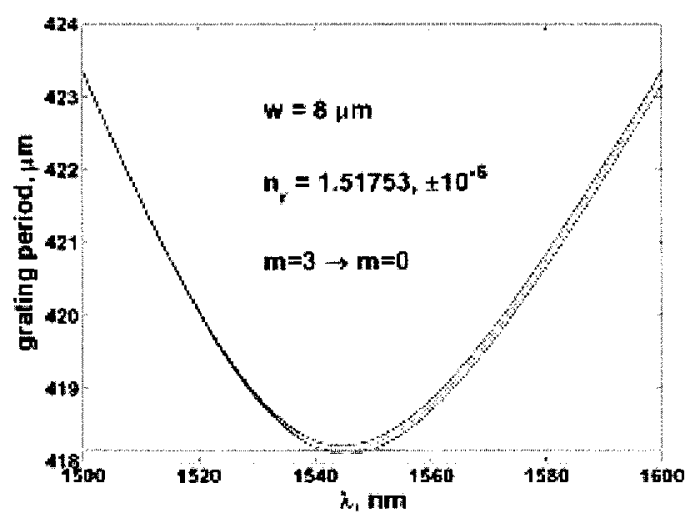

c)

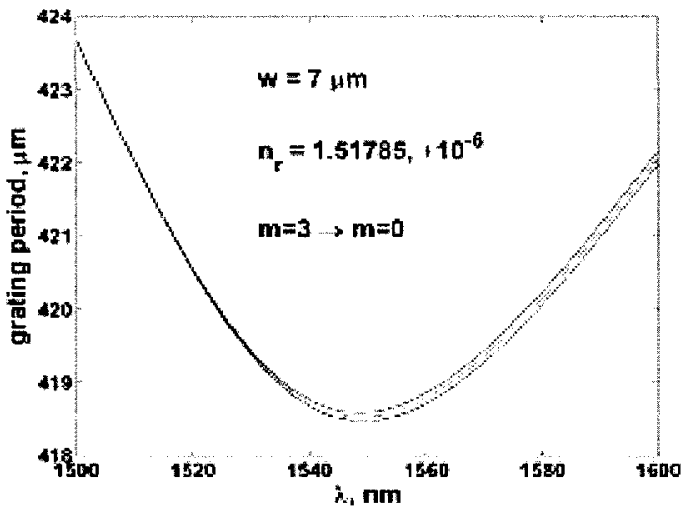

b)

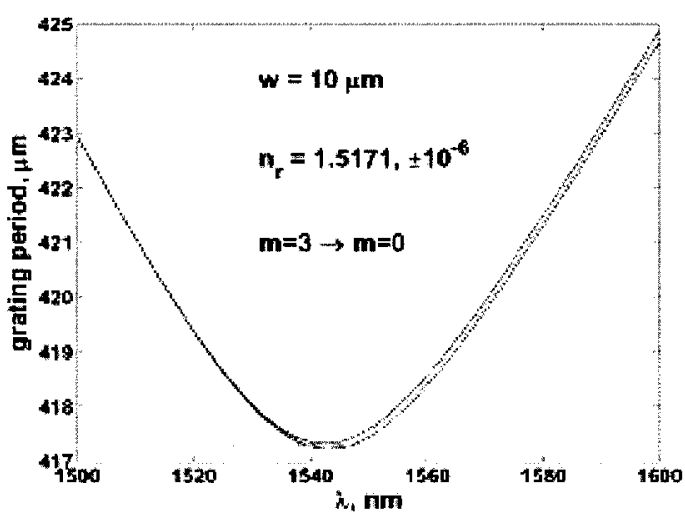

d)

Fig. 4-14. Plot $\Lambda=\frac{\lambda}{n_{\text {eff } 0}-n_{\text {eff } 3}}$ versus wavelength for various widths of the core.

Parameters: $\mathrm{v}=25 \mu \mathrm{m}, \mathrm{w}=4.5 \mu \mathrm{m}, \mathrm{s}=5 \mu \mathrm{m}, n_{c}=1.52, n_{b}=1.51$.

a) $n_{r}=1.5195-10^{-6}$ (bottom), $n_{r}=1.5195$ (middle), $n_{r}=1.5195+10^{-6}$ (top), $\mathrm{w}=4.5 \mu \mathrm{m}$.

b) $n_{r}=1.51785-10^{-6}$ (bottom), $n_{r}=1.51785$ (middle),

$n_{r}=1.51785+10^{-6}$ (top), $\mathrm{w}=7 \mu m$ 
c) $n_{r}=1.51753-10^{-6}$ (bottom),

$n_{r}=1.51753$ (middle), $n_{r}=1.51753+10^{-6}$ (top), $\mathrm{w}=8 \mu m$

d) $n_{r}=1.5171-10^{-6}$ (bottom), $n_{r}=1.5171$ (middle), $n_{r}=1.5171+10^{-6}$ (top), $\mathrm{W}=10 \mu \mathrm{m}$

Fig. 4-14a-d show the phase matching curves for different widths, $w$ of the second channel. Smaller width results in weaker $\lambda$-dependence.

Fig. 4-15a, b show the phase matching curves for a wide core $(\mathrm{w}=8,10 \mu \mathrm{m})$ and coupling between mode $m=2$ and $m=0$. Fine tuning by choosing optimum initial value of the core refractive index allows to achieve widely separated dual peak resonance as seen in Fig. 4$15 \mathrm{a}$.

Fig. 4-16a-d show the phase matching curves for different widths, $v$ of the first channel. For a wider channel the total number of modes increases (whose fields are concentrated mostly in the first, wide channel), but the transition slope stays the same. Matching curves are presented in Fig. 4-17a-d. when two resonance conditions are far apart (Fig. 4-17b), the limiting value for the coupling coefficient is, $\kappa=\frac{1.42}{\sqrt{3}} \approx 0.82 \mathrm{~m}^{-1}$ and the $100 \%$ coupling length is $L_{g}=\frac{\pi}{2 \kappa}=\frac{\pi}{2 \times 0.82} \approx 1.916 \mathrm{~m}$. 


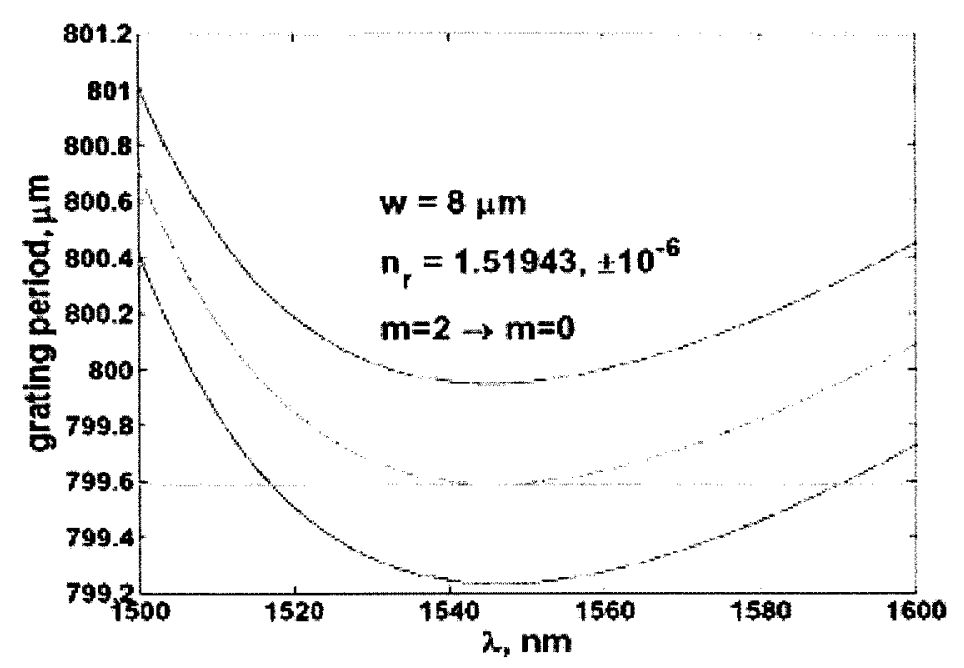

a)

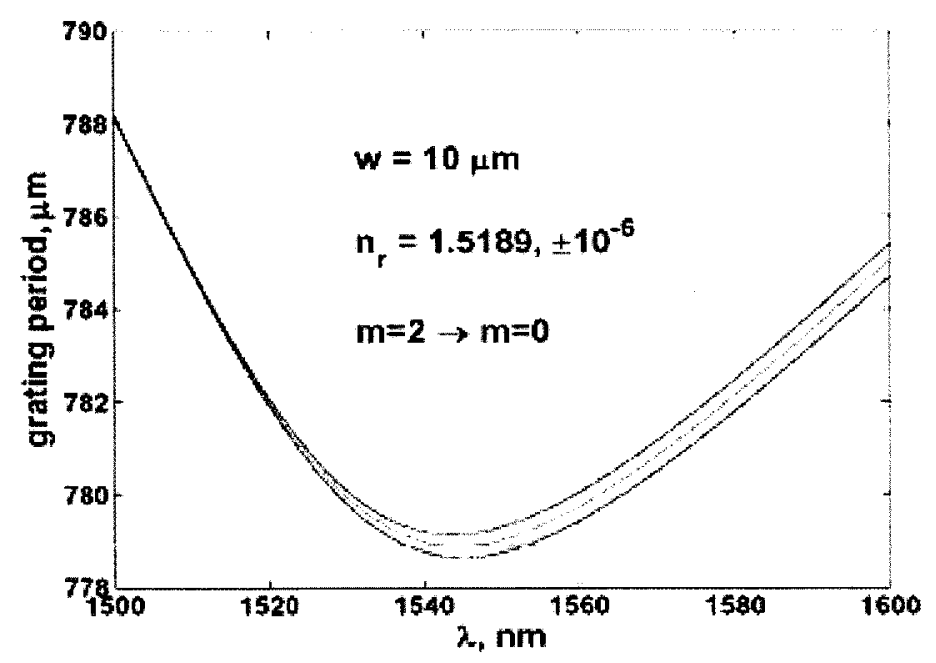

b)

Fig. 4-15. Plot $\Lambda=\frac{\lambda}{n_{\text {eff } 0}-n_{\text {eff } 2}}$ versus wavelength for three values of core refractive indeces. Parameters: $\mathrm{v}=25 \mu \mathrm{m}, \mathrm{s}=5 \mu \mathrm{m}, n_{c}=1.52, n_{b}=1.51$.

a) $n_{r}=1.51943-10^{-6}$ (bottom), $n_{r}=1.51943$ (middle), $n_{r}=1.51943+10^{-6}$ (top), $\mathrm{w}=8 \mu \mathrm{m}$.

b) $n_{r}=1.5189-10^{-6}$ (bottom), $n_{r}=1.5189$ (middle), $n_{r}=1.5189+10^{-6}$ (top), $\mathrm{w}=10 \mu \mathrm{m}$ 


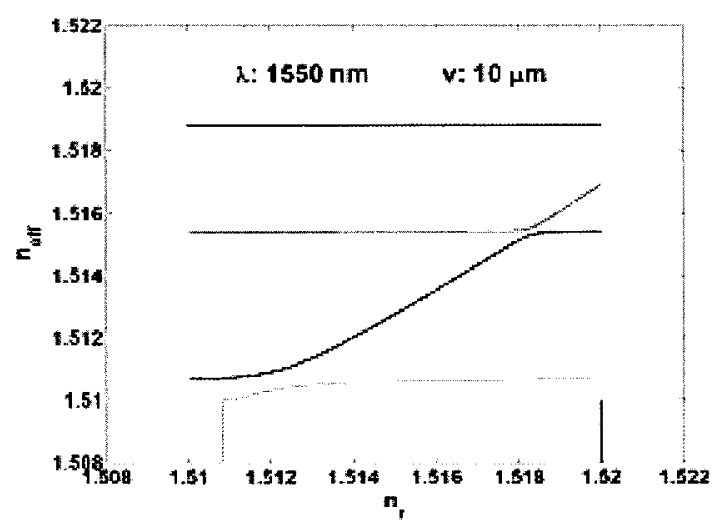

a)

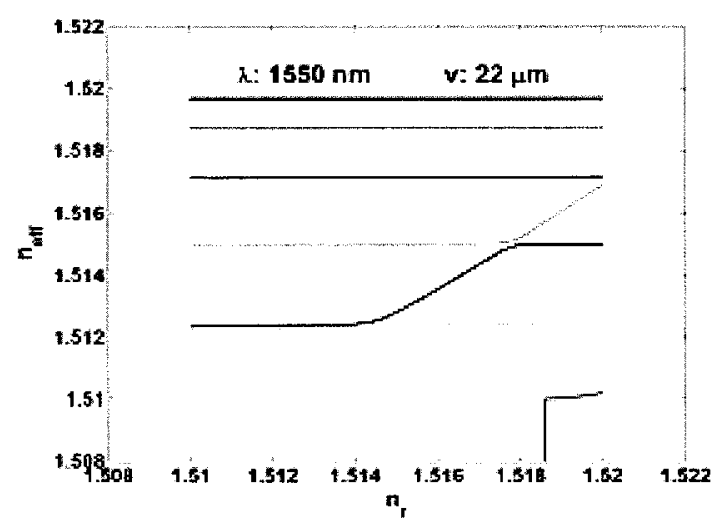

c)

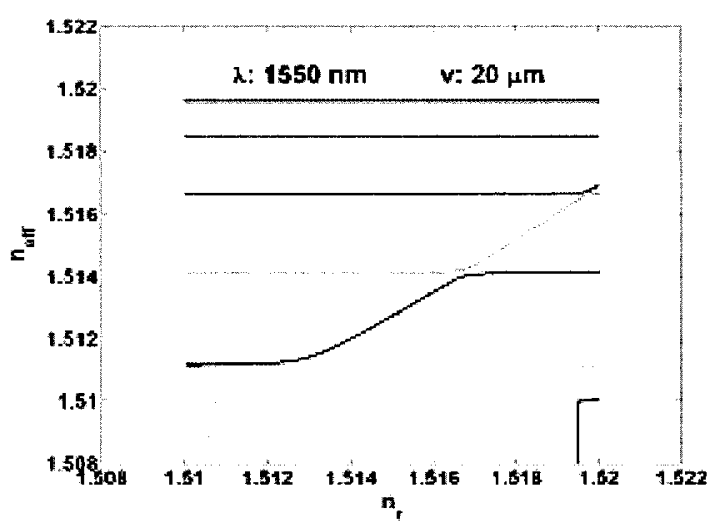

b)

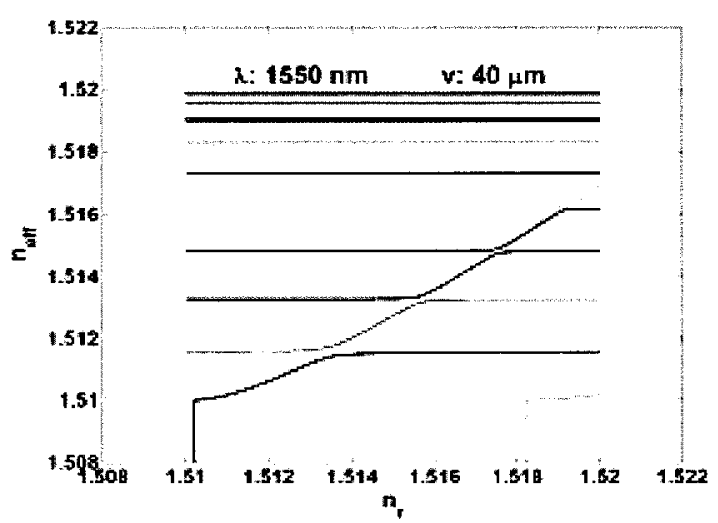

d)

Fig. 4-16. Modal indices versus core refractive index $n_{r}$ for various cladding layer widths. Parameters: $\mathrm{w}=25 \mu \mathrm{m}, \mathrm{s}=5 \mu \mathrm{m}, n_{c}=1.52, n_{b}=1.51, \lambda=1550 \mathrm{~nm}$
a) $\mathrm{v}=10 \mu \mathrm{m}$
b) $\mathrm{v}=20 \mu \mathrm{m}$
c) $\mathrm{v}=22 \mu \mathrm{m}$
d) $\mathrm{v}=40 \mu \mathrm{m}$ 


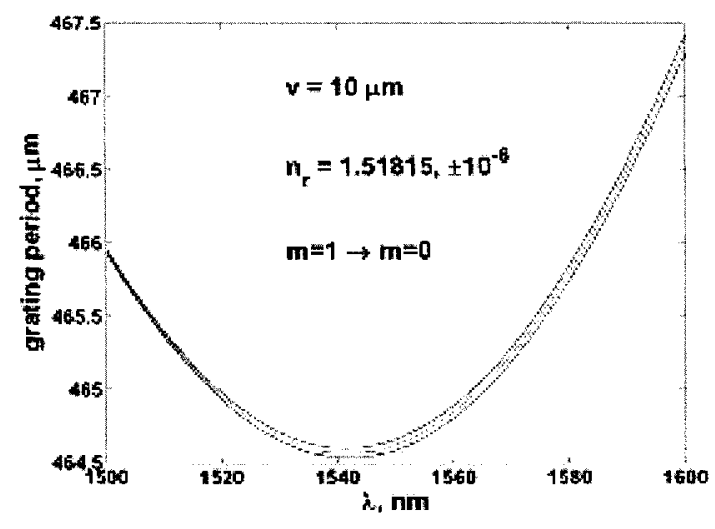

a)

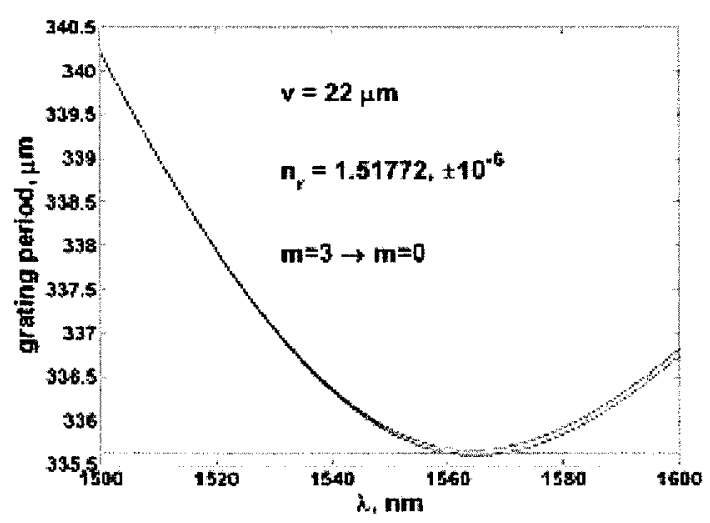

c)

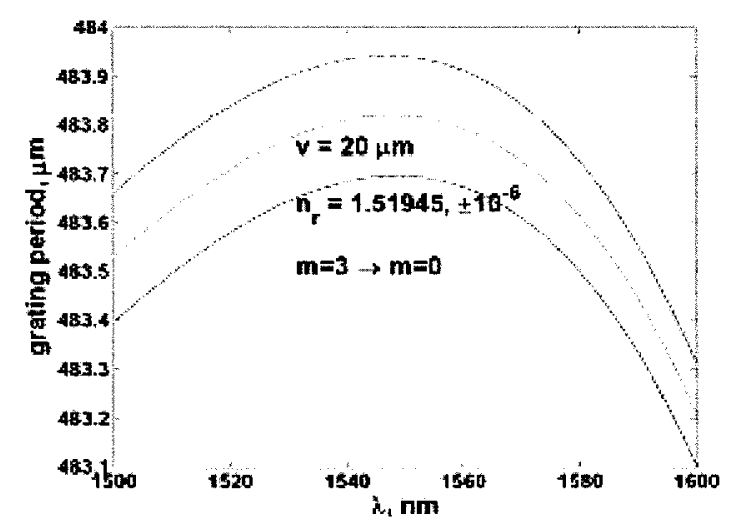

b)

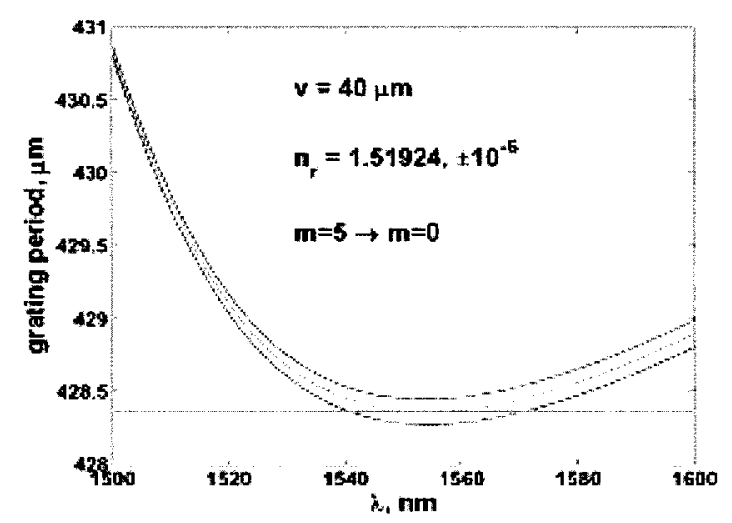

d)

Fig. 4-17. Plot $\Lambda=\frac{\lambda}{n_{\text {eff } 0}-n_{\text {eff_m }}}$ versus wavelength for various cladding layer widths and mode coupling. Parameters:, $\mathrm{w}=5 \mu \mathrm{m}, \mathrm{s}=5 \mu \mathrm{m}, n_{c}=1.52, n_{b}=1.51$. a) $\mathrm{v}=10 \mu \mathrm{m}, n_{r}=1.51815-10^{-6}$ (bottom), $n_{r}=1.51815$ (middle), $n_{r}=1.51815+10^{-6}$ (top), $m=1$ b) $\mathrm{v}=20 \mu \mathrm{m}, n_{r}=1.51945-10^{-6}$ (bottom), $n_{r}=1.51945$ (middle), $n_{r}=1.51945+10^{-6}$ (top), $m=3$

c) $\mathrm{v}=22 \mu \mathrm{m}, n_{r}=1.51772-10^{-6}$ (bottom), $n_{r}=1.51772$ (middle), $n_{r}=1.51772+10^{-6}$ (top), $m=3$ 
d) $\mathrm{v}=40 \mu \mathrm{m}, n_{r}=1.51924-10^{-6}$ (bottom), $n_{r}=1.51924$ (middle),

$$
n_{r}=1.51924+10^{-6} \text { (top), } m=5
$$

Fig. 4-18a-c shows modal indices for different separations between two channels. As the separation gets larger, fields are distributed either in channel one or in channel two and modal transitions are sharper, but steady state values of modal indices are about the same, they are not getting closer towards $n_{c}$. The phase matching curves for coupling between mode $m=3$ and mode $m=0$ for a fixed value of the core refractive index, $n_{r}=1.519$, $n_{r}=1.519 \pm 10^{-6}$ and scanned separation is shown in Fig. 4-19a-c. We observe a weaker $\lambda-$ dependence for smaller separation. 


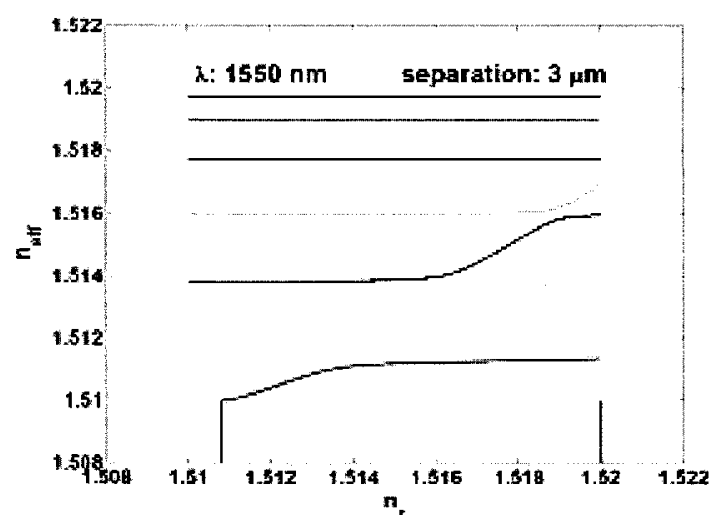

a)

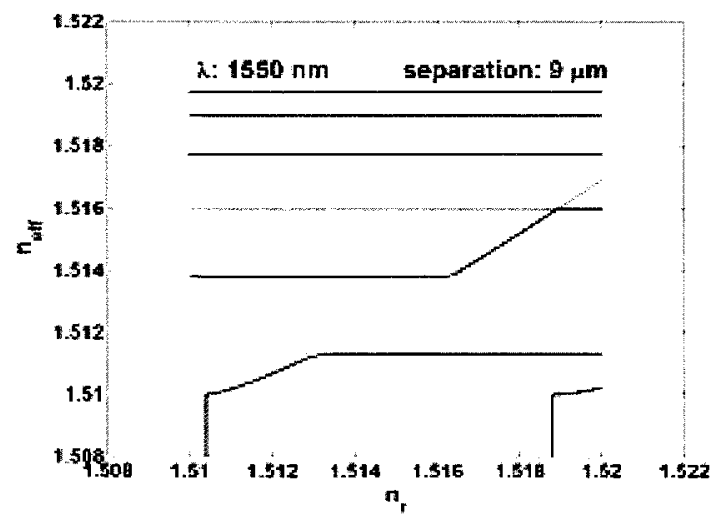

c)

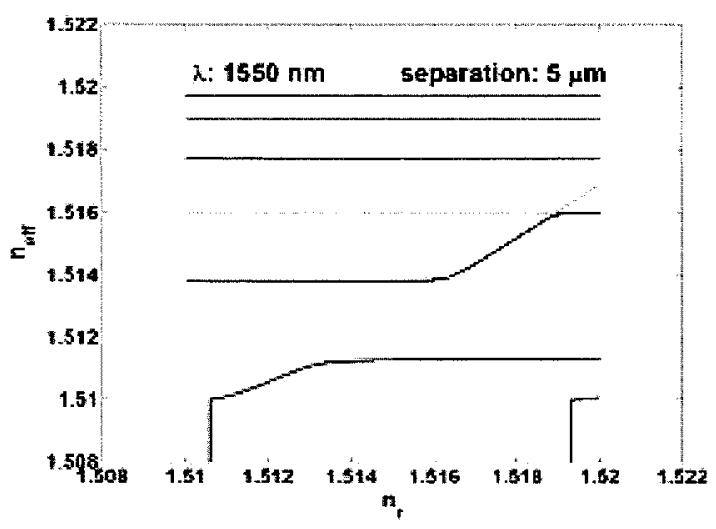

b)

Fig. 4-18. Modal indices versus core refractive index $n_{r}$ for various separations.

Parameters: $\mathrm{v}=25 \mu \mathrm{m}, \mathrm{w}=5 \mu \mathrm{m}, n_{c}=1.52, n_{b}=1.51, \lambda=1550 \mathrm{~nm}$
a) $\mathrm{s}=3 \mu \mathrm{m}$
b) $\mathrm{s}=5 \mu \mathrm{m}$
c) $\mathrm{s}=9 \mu \mathrm{m}$ 


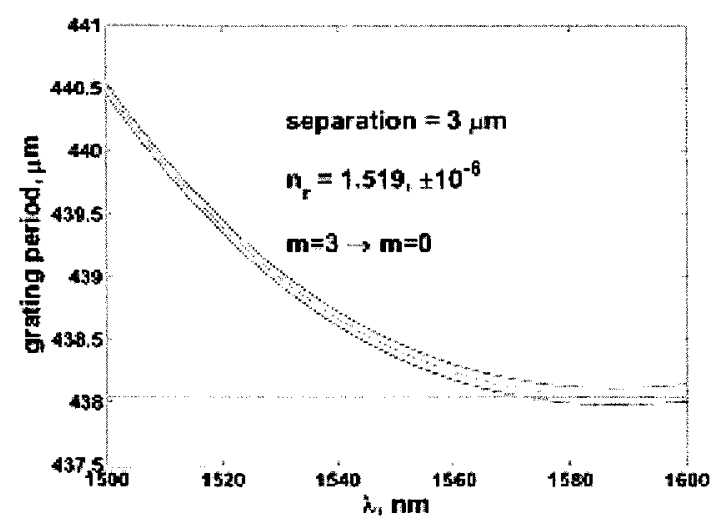

a)

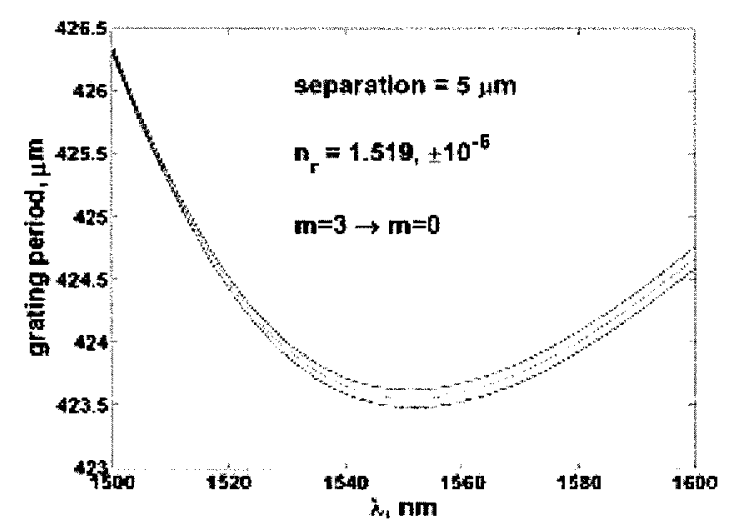

b)

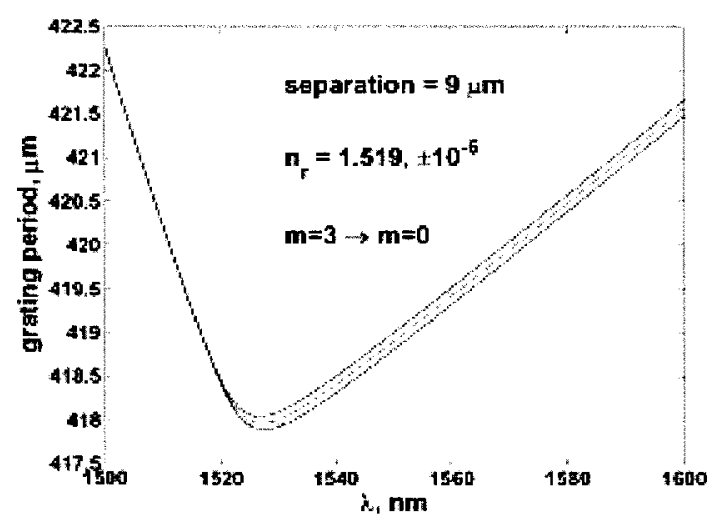

c)

Fig. 4-19. Plot $\Lambda=\frac{\lambda}{n_{\text {eff } 0}-n_{\text {eff } 3}}$ versus wavelength for various separations. Parameters:

$\mathrm{v}=25 \mu \mathrm{m}, \mathrm{W}=5 \mu \mathrm{m}, n_{c}=1.52, n_{b}=1.51, \lambda=1550 \mathrm{~nm}$

a) $\mathrm{s}=3 \mu \mathrm{m}, n_{r}=1.519-10^{-6}$ (bottom), $n_{r}=1.519$ (middle),

$n_{r}=1.519+10^{-6}$ (top).

b) $\mathrm{s}=5 \mu \mathrm{m}, n_{r}=1.519-10^{-6}$ (bottom), $n_{r}=1.519$ (middle),

$n_{r}=1.519+10^{-6}$ (top).

c) $\mathrm{s}=9 \mu \mathrm{m}, n_{r}=1.519-10^{-6}$ (bottom), $n_{r}=1.519$ (middle),

$n_{r}=1.519+10^{-6}$ (top). 
The limiting value for the coupling coefficient with $\mathrm{s}=3 \mu \mathrm{m}, n_{r}=1.5191$ is, $\kappa=\frac{1.17}{\sqrt{3}} \approx 0.675 \mathrm{~m}^{-1}$ and the $100 \%$ coupling length is $L_{g}=\frac{\pi}{2 \kappa}=\frac{\pi}{2 \times 0.675} \approx 2.327 \mathrm{~m}$ (phase matching plot in Fig. 4-20a). The limiting value for the coupling coefficient with $\mathrm{s}=3 \mu \mathrm{m}$, $n_{r}=1.5192$ is, $\kappa=\frac{1.265}{\sqrt{3}} \approx 0.73 \mathrm{~m}^{-1}$ and the $100 \%$ coupling length is $L_{g}=\frac{\pi}{2 \kappa}=\frac{\pi}{2 \times 0.73} \approx 2.152 \mathrm{~m}$ (phase matching plot in Fig. 4-20b). A length of the order of two meters for planar waveguide is too large for practical applications. Later we will examine a possibility to reduce the length. 


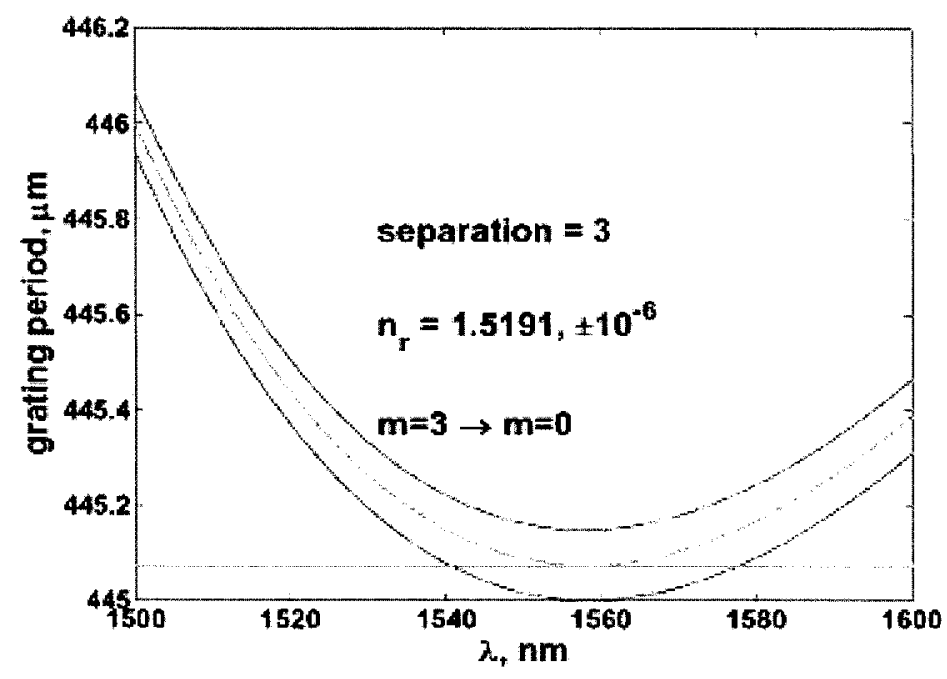

a)

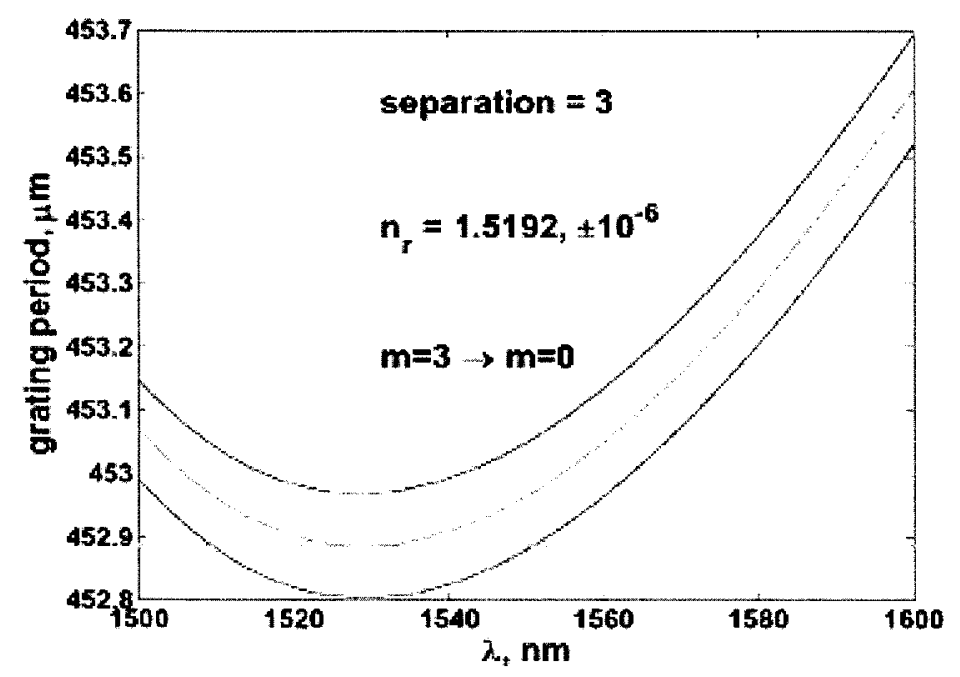

b)

Fig. 4-20. Plot $\Lambda=\frac{\lambda}{n_{\text {eff } 0}-n_{\text {eff } 3}}$ versus wavelength for two initial values of core refractive index. Parameters: $\mathrm{v}=25 \mu \mathrm{m}, \mathrm{w}=5 \mu \mathrm{m}, \mathrm{s}=3 \mu \mathrm{m}, n_{c}=1.52$, $n_{b}=1.51$.
a) $n_{r 0}=1.5191, n_{r}: n_{r 0}-10^{-6}, n_{r 0}, n_{r 0}+10^{-6}$
b) $n_{r 0}=1.5192, n_{r}: n_{r 0}-10^{-6}, n_{r 0}, n_{r 0}+10^{-6}$ 
By varying each of the geometrical parameters we can optimize the phase matching curves to make them flat along the $\lambda$-axis. A general procedure to achieve this is the following. First we change the width of the first channel, $v$ and the width of the second channel, $w$ to push the 'turning' point towards higher modes. By doing this we minimize the $\lambda$-slope of the 'plateau'. Then we reduce the separation between two channels, $s$ to make the 'edge' between 'plateau' and 'steep descent' smooth, so the whole path of operation lies in the smooth 'edge' and does not touch regions of 'plateau' and 'steep descent'. Finally, the influence of the refractive index of the first channel on modal indices has been investigated, it is shown in Fig. 4-21a-c. 


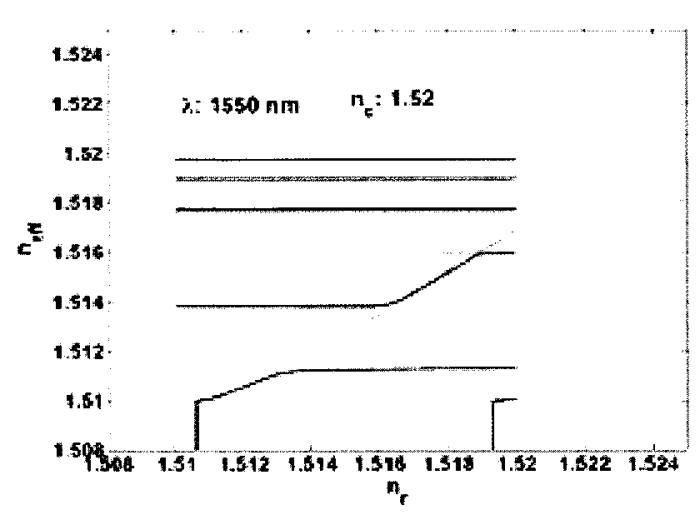

a)

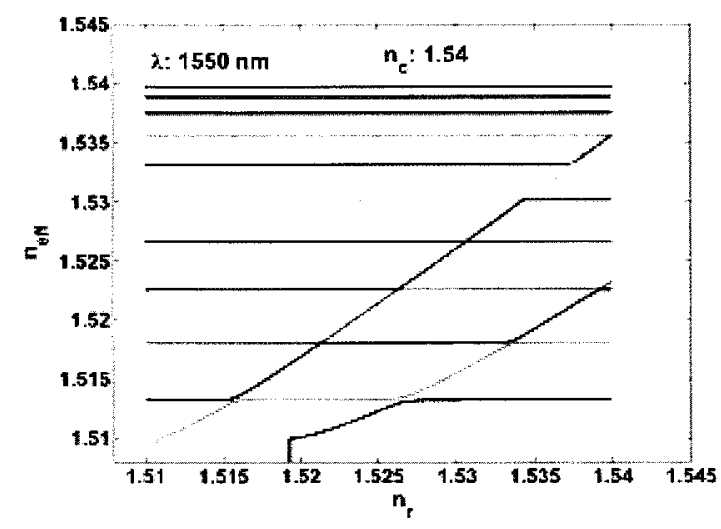

c)

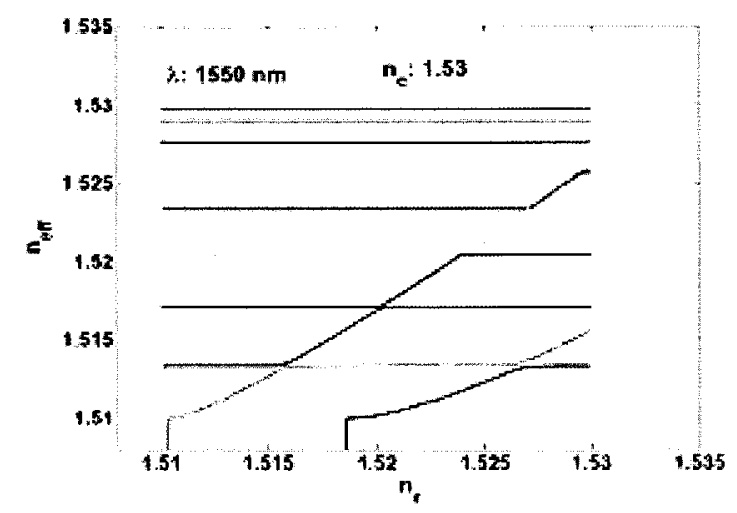

b)

Fig. 4-21. Modal indices versus core refractive index $n_{r}$ for various cladding refractive indeces $n_{c}$. Parameters: $\mathrm{v}=25 \mu \mathrm{m}, \mathrm{w}=5 \mu \mathrm{m}, \mathrm{s}=5 \mu \mathrm{m}, n_{b}=1.51$, $\lambda=1550 \mathrm{~nm}$
a) $n_{c}=1.52$
b) $n_{c}=1.53$
c) $n_{c}=1.54$ 
When the transmission spectrum is optimized to be sensitive to small nonlinear effects, i.e. to small refractive index modulations, $\Delta n_{r}=n_{2}|E|^{2}$ due light intensity modulations, it has some negative impact too. The spectrum becomes also sensitive to the refractive index deviation $\left(\Delta n_{r_{-} \text {FABRICATION }}\right.$ ) due technological parameters fluctuations in the process of waveguide fabrication. With our previous example we want to estimate the effect of such deviation on the transmission characteristics when $\Delta n_{r_{-} \text {FABRICATION }}= \pm 10^{-4}$. Phase matching curves of the device with $n_{r 0}=1.519 \pm 10^{-1}$ are shown in Fig. 4-22a, b. In both cases the minimal grating period has moved outside the range $1520-1570 \mathrm{~nm}$, which corresponds to the fluorescent bandwidth of $\mathrm{Er}^{3+}$-ions. 


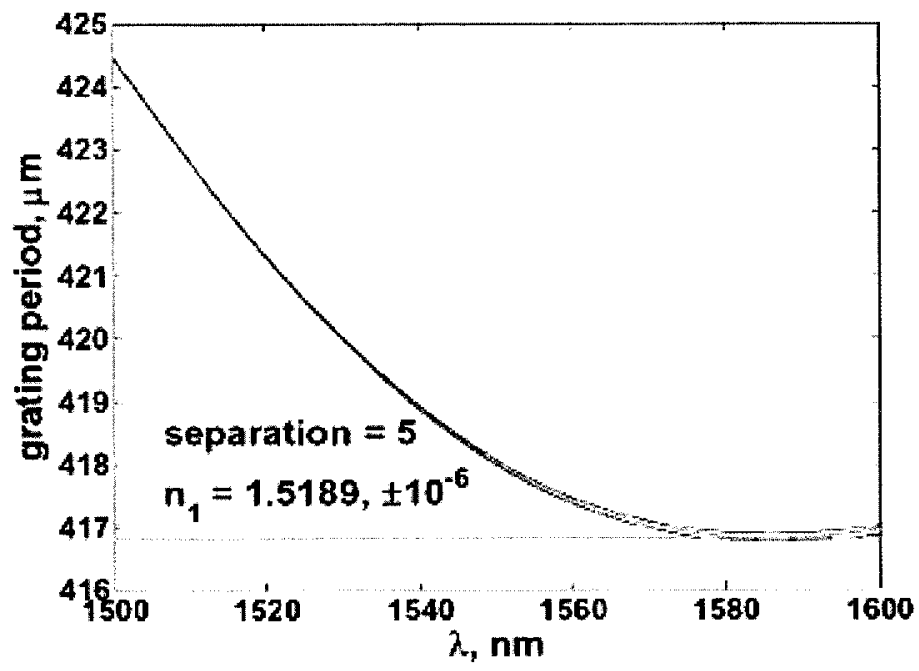

a)

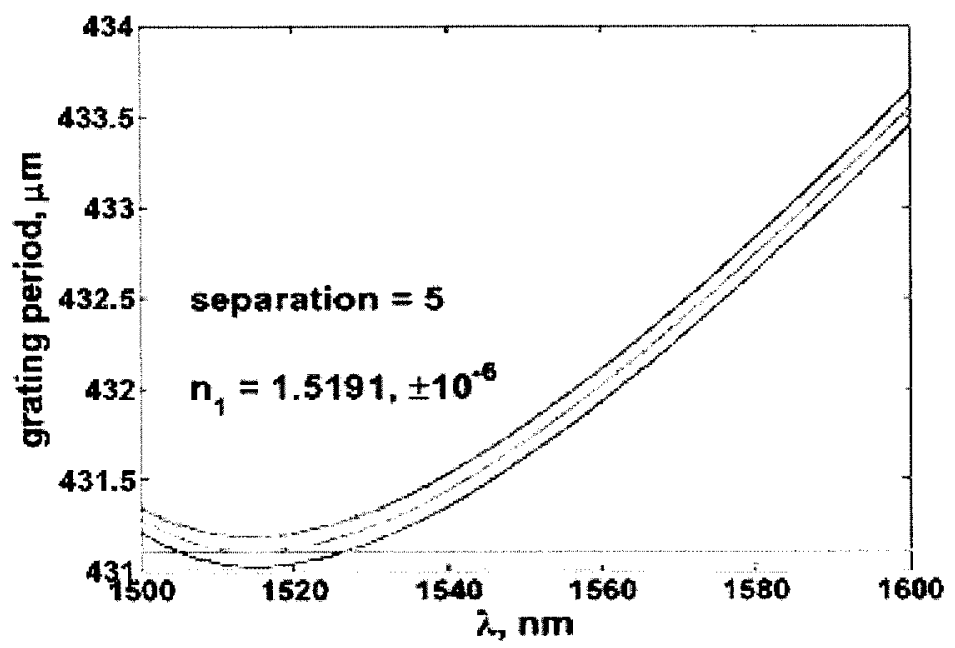

b)

Fig. 4-22. Plot $\Lambda=\frac{\lambda}{n_{\text {eff } 0}-n_{\text {eff } 3}}$ versus wavelength for two initial values of core refractive index. Parameters: $\mathrm{v}=25 \mu \mathrm{m}, \mathrm{w}=5 \mu \mathrm{m}, \mathrm{s}=5 \mu \mathrm{m}, n_{c}=1.52$, $n_{b}=1.51$.

a) $n_{r 0}=1.5189, n_{r}: n_{r 0}-10^{-6}, n_{r 0}, n_{r 0}+10^{-6}$

b) $n_{r 0}=1.5191, n_{r}: n_{r 0}-10^{-6}, n_{r 0}, n_{r 0}+10^{-6}$ 


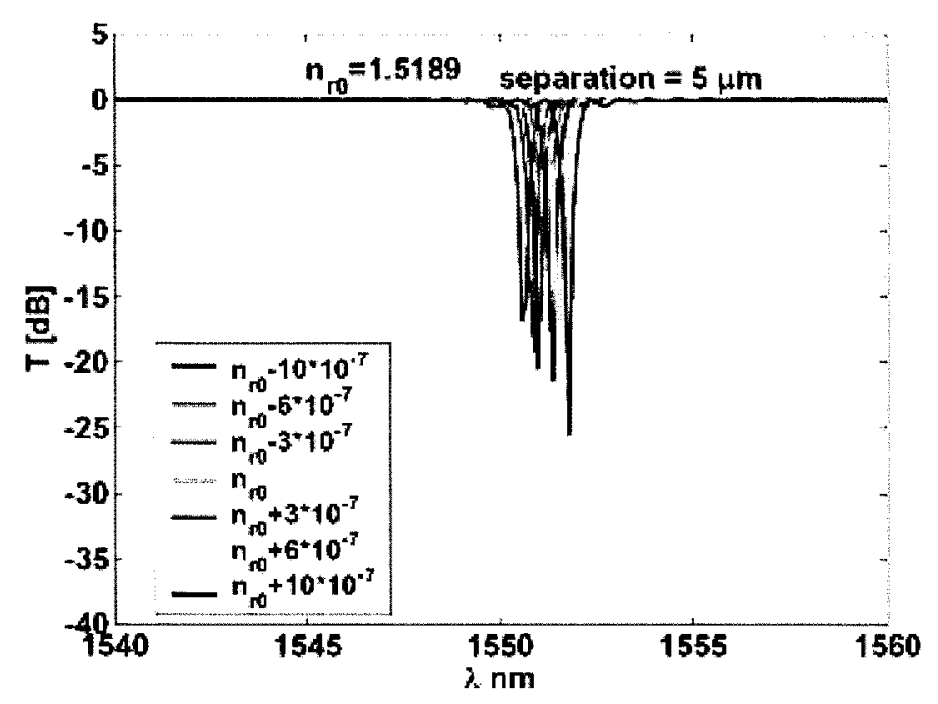

a)

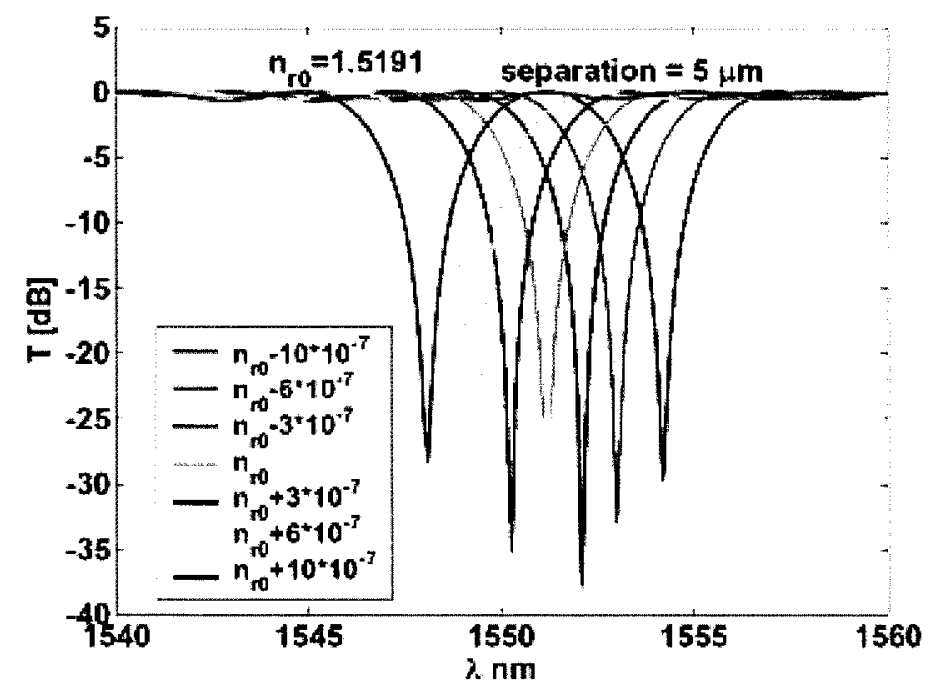

b)

Fig. 4-23. Transmission characteristic wavelength for two initial values of core refractive index. Parameters: $\mathrm{v}=25 \mu \mathrm{m}, \mathrm{w}=5 \mu \mathrm{m}, \mathrm{s}=5 \mu \mathrm{m}, n_{c}=1.52$, $n_{b}=1.51$.
a) $n_{r 0}=1.5189, n_{r}: n_{r 0}-10^{-6}, n_{r 0}, n_{r 0}+10^{-6}$
b) $n_{r 0}=1.5191, n_{r}: n_{r 0}-10^{-6}, n_{r 0}, n_{r 0}+10^{-6}$ 
Therefore, if we want the central resonance to be at the previous position, i.e. at $\lambda_{0} \approx 1551 \mathrm{~nm}$, then transmission spectrum, shown in Fig. 4-23a, b, has no longer a dual peak resonance near that wavelength. The situation might be improved if we choose a smaller separation parameter, $\mathrm{s}=3 \mu \mathrm{m}$ (instead of $\mathrm{s}=5 \mu \mathrm{m}$ ). As we have seen earlier, when two channels are closer to each other the phase matching curves have weaker $\lambda$-dependence and therefore the dual peaks are separated wider along $\lambda$-axis. The transmission spectrum for this case is shown in Fig. 4-24. It still exhibits dual peak resonance in the range $1520-1570$ $\mathrm{nm}$.

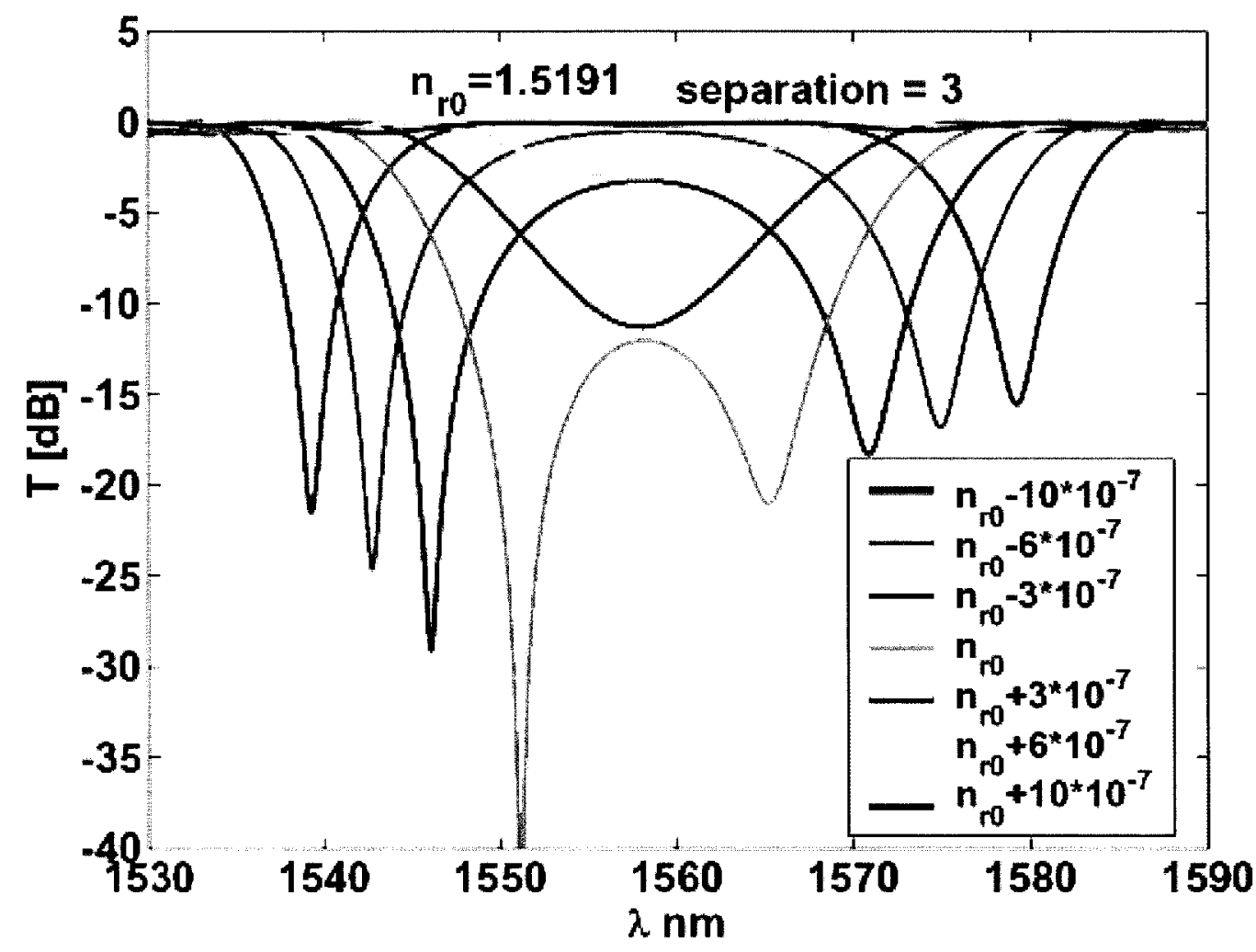

Fig. 4-24. Transmission characteristic for initial value of core refractive index 1.5191, $n_{r 0}=1.5191$. Parameters: $\mathrm{v}=25 \mu \mathrm{m}, \mathrm{w}=5 \mu \mathrm{m}, \mathrm{s}=3 \mu \mathrm{m}, n_{c}=1.52$, $n_{b}=1.51, \lambda=1550 \mathrm{~nm}$ 
As a final comment, if $\Delta n_{r_{-} F A B R I C A T I O N}$ is very small and comparable with nonlinear effects, i.e. $\triangle n_{r_{-} \text {FABRICATION }} \approx \pm 10^{-6}$, then we can compensate for such deviation with an additional light intensity $I_{F}$ :

$$
\begin{aligned}
& n_{r}=n_{r 0}+n_{2} I \rightarrow n_{r}=n_{r 0}+\Delta n_{F}+n_{2}\left(I+I_{F}\right)=n_{r 0}+n_{2} I+\left(\Delta n_{F}+n_{2} I_{F}\right)= \\
& =n_{r 0}+n_{2} I \text { if } \Delta n_{F}+n_{2} I_{F}=0 \text { or } I_{F}=-\frac{\Delta n_{F}}{n_{2}} .
\end{aligned}
$$

\subsection{Summary and discussions}

The LPG element (slab coupler with long period grating) can be used as a controllable filter. If $n_{r}$ is controlled externally (by an active LPG element) the modulation $\delta n_{r}=\delta\left(n_{r 2}\right)-\delta\left(n_{r 1}\right)$ can be large. Then the coupling coefficient can be chosen to be large too, $\kappa \approx \frac{\delta\left(n_{r 2}\right)-\delta\left(n_{r 1}\right)}{\sqrt{3}}$. The total length of element will be relatively small, for instance $\sim 5 \mathrm{~cm}$.

Another way to control $n_{r}$ is by light intensity modulation. When the second channel for instance is doped by $\mathrm{Er}^{3+}$, high pumping light intensity at $980 \mathrm{~nm}$ or $1480 \mathrm{~nm}$ can invert the population (i.e. number of atoms in an excited states is larger than number of atoms in the ground states) and therefore modify the refractive index slightly. Assuming, that the refractive index modulation in this case is of the order $\delta n_{r} \approx 10^{-6}$, as discussed in chapter 1 , the maximum coupling then should be relatively small, $\sim 0.7 \mathrm{~m}^{-1}$ and the total length of LPG is large, $\sim 2.194 \mathrm{~m}$.

From the output point of view a dip in the transmission characteristic may be regarded as an absorption. Controlling the dip by modulation of the core refractive index, $n_{r}$ then makes the LPG element a controllable absorber. To use such an element for laser modelocking requires 
that the element must be fast, i.e. absorption must be controlled (modified) in the time scale of one round trip of the light in the laser cavity. We have seen that placing a LPG slab into the cavity might preserve the original LPG transmission function, but it does not make the coupling larger, therefore the cavity should be $\sim 2.194 \mathrm{~m}$ or more which is not practical. One way to save space is to use a spiral configuration with bend radius much larger than the width of the channel, as shown in Fig. 4-25. However, to fabricate a spiral shape that avoids selfcrossing is difficult. A long optical waveguide with several bends was fabricated on a 5 inch Si wafer [58]. A minimum loss of $3.5 \mathrm{~dB} / \mathrm{m}$ was obtained in a $1.6 \mathrm{~m}$ long waveguide at a wavelength $1.55 \mu \mathrm{m}$.

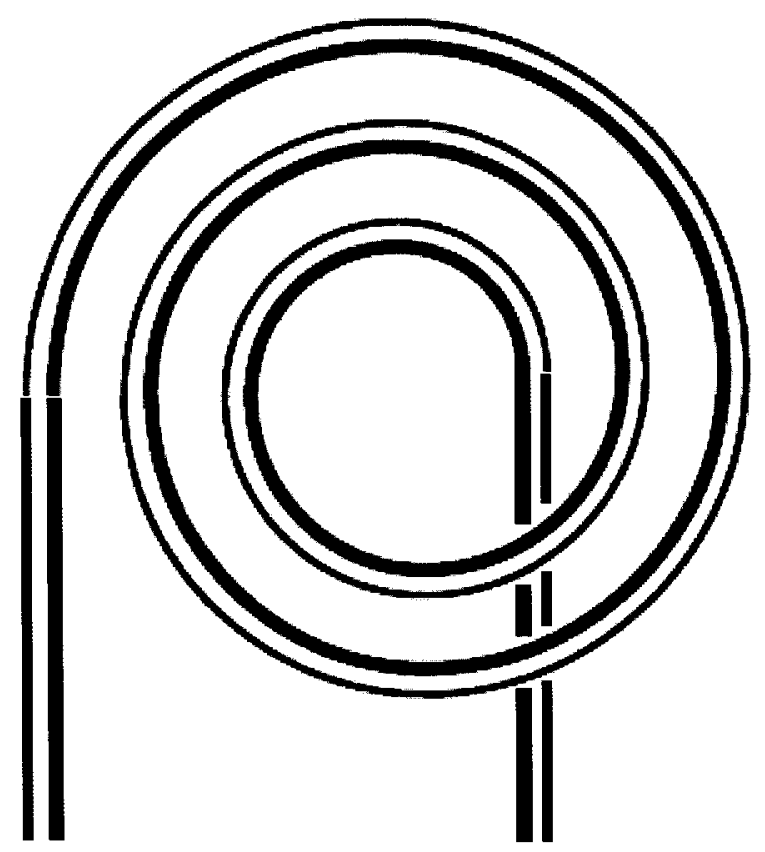

Fig. 4-25. LPG slab made with spiral configuration. 
Using a material with a larger nonlinear refractive index, $n_{2}$ will also increase the coupling and reduce the total length of the LPG. 


\section{Chapter 5}

\section{Discussion and Conclusions}

1. A five-layer non-symmetrical slab waveguide was analyzed. We have applied a perturbation approach to the characteristic equation and have found an analytical approximate expression for the effective modal index (and propagation constant) as a function of geometrical and material parameters. This result has a relatively good agreement with the effective index obtained by using numerical simulation with the software package $\mathrm{C} 2 \mathrm{~V}$. The geometrical structure of the five-layer slab is similar to a fiber with an additional coating that has refractive index higher than in the cladding, shown in Fig. 5-1a. Such fiber with a long period grating was analyzed recently with a numerical method based on long period mode approximation and coupled mode theory [42]. A graph of the effective indices versus deposition thickness, $d$ is shown in Fig. 5-1b. This graph has special points corresponding to a modal transition from one steady state to the next, as in the plot of the effective indices versus material refractive index, $n_{r}$ that we obtained on Fig. 4-2a. Functional dependences $n_{e f f}$ vs. $d$ and $n_{e f f}$ vs. $n_{r}$ are similar, because the effective index in general depends on the transverse optical path, $d \cdot n_{r}$. An agreement with [42] indirectly validates our result in Fig. 4-2a. (thickness, $d$ and material refractive index, $n_{r}$ enter characteristic equation (eigenvalue equation) together as a product $d \cdot n_{r}$, which has a physical meaning of the transverse optical path, therefore functional dependences $n_{\text {eff }}$ vs. $d$ and $n_{e f f}$ vs. $n_{r}$ should be similar)

2. We have found a special (sensitive) value of the core refractive index, $n_{r 0}$. Near $n_{r 0}$ the phase matching curve changes slope along the $\lambda$-axis from positive to negative. The turning point, where the slope is zero, can be found for any mode. Each of the geometrical parameters (widths of both channels $(w, v)$, separation $(s)$ between them) and $n_{r}$ has its 
effect on the phase matching curves. By tailoring $w, v, s, n_{r}$ the phase matching curves can be made 'flat' (weak $\lambda$-dependence), monotonically decreasing or increasing over a wide range of wavelengths. In addition, they can be made very sensitive to $n_{r}$-modulation, or if we desire insensitive.

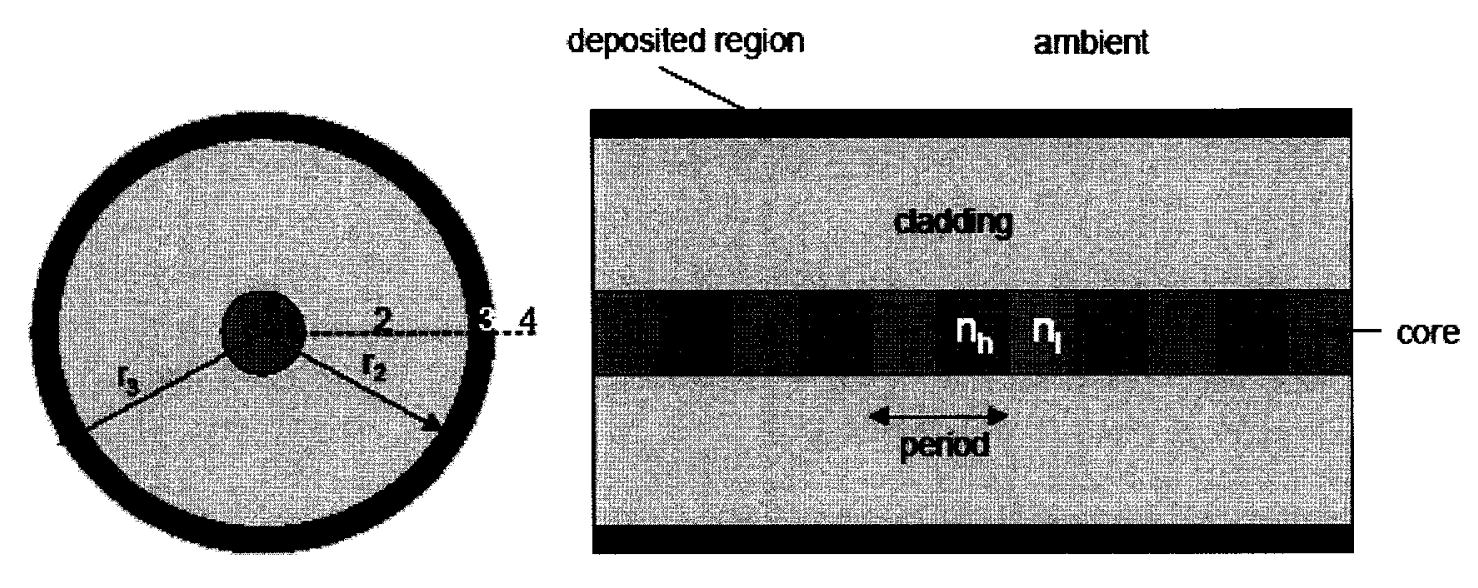

Fig. 5-1a. Transversal and longitudinal section of the fiber with an additional layer deposited on the cladding. Refractive index of the deposited layer is higher than refractive index of the cladding. [42] 


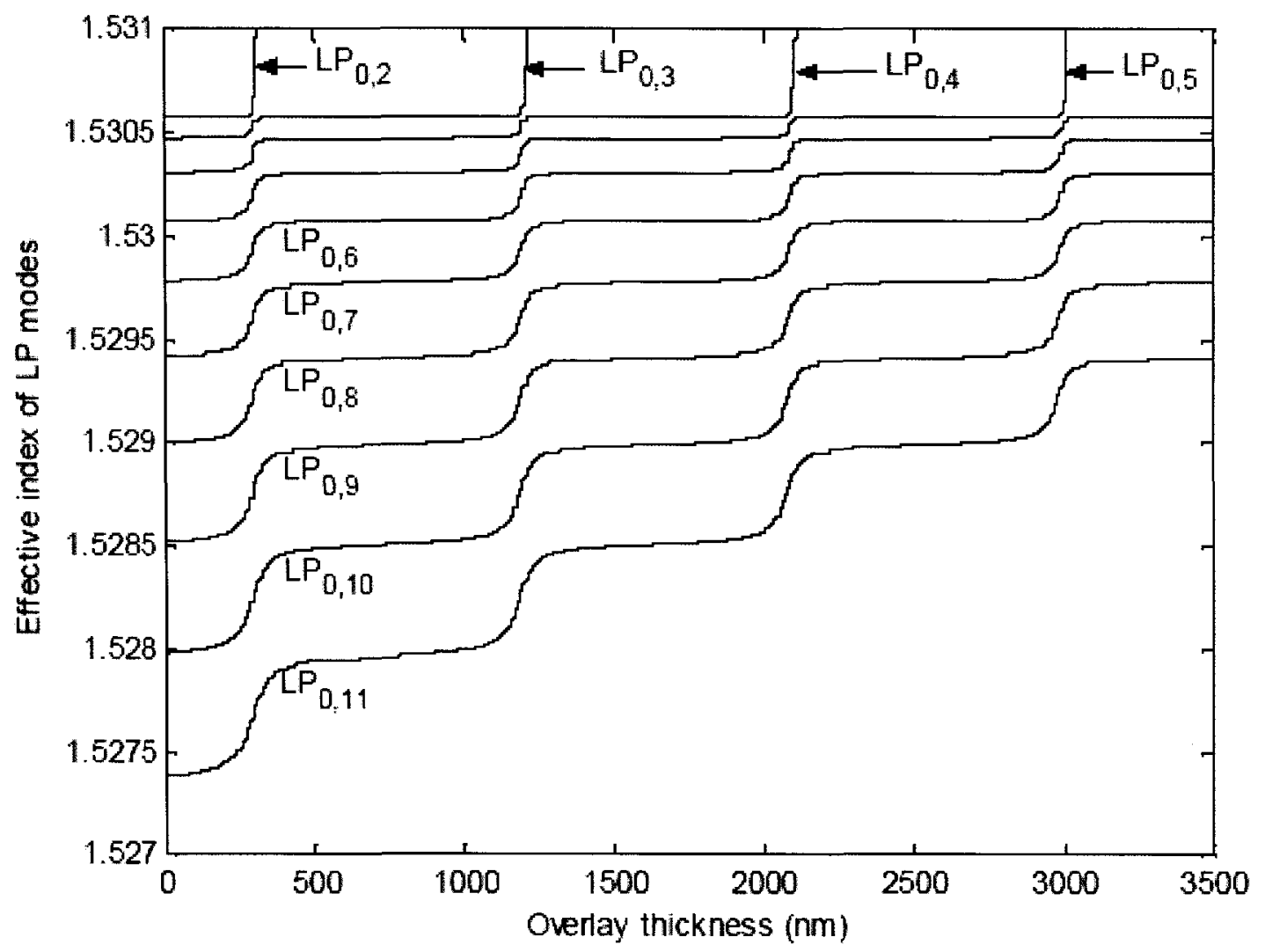

Fig. 5-1b. Effective index as a function of the overlay thickness of cladding modes. [42]

This has an impact on the transmission characteristics of the five-layer slab with long period grating:

- we can control the direction and shift (due to $n_{r}$-modulation) of the resonance wavelength, $\lambda_{0}$ corresponding to the loss peak

- we can control the value of $\lambda_{0}$ and make it coincide with the fluorescence peak of rare-earth doped element, for $\mathrm{Er}^{3+} \sim 1550 \mathrm{~nm}$ 
- we can also control the width of the attenuation band to cover the width of the fluorescence band $\mathrm{Er}^{3+} \sim 40 \mathrm{~nm}$

3. A possible application is as a tunable optical filter in a dynamic DWDM system and network, [43]. Since our filter can be controlled optically it can be used in all optical network which has an advantage of transferring data without unwanted signal conversion from an optical form to an electrical and back to an optical.

If the refractive index modulation is achieved with thermooptic effect, then the filter is relatively slow with switching time up to few milliseconds. Such filter can be suitable for so called circuit switching. When an index modulation is due to electrooptic effect or light intensity the filter is fast with switching time from microseconds to nanoseconds. These filters may be candidates for packet switching which arguably has more advantages. The circuit switching type networking requires, before transferring any data, establishing a dedicated path (called a circuit) between a sender and a receiver through many nodes in the network.

In the case of packet switching, no path between a sender and receiver is established in advance. Information flows are broken into variable-size packets. Each packet consists of an actual data to be transferred and the packet header which contains sender and receiver address and some other information. These packets are sent, one by one, to the nearest router, which will look up the destination address, store them temporarily (buffered), and then forward them to the corresponding next node. Processing at a router node involves determining destination node for a packet, verifying whether or not a given packet has been already received and buffered and calculating the shortest path for forwarding a packet.

Packet switching can accept a greater number of simultaneously active users than circuit switching. Probably because of this it is considered that packet switching is more suitable for the Internet.

A packet switched network can not be implemented right now with all optical components, because there is no easy way to store (buffer) optical signal and do some simple processing as with the electrical signal. 
We have to note that DWDM normally requires pass band type of filters rather than stop band. An ordinary LPG by its nature is a stop band filter. Few proposals are made to turn the LPG fiber into a pass band filter by implementing $\pi$-phase shift in the grating, see [44], [45]. However, in the case of dual peak resonance, the LPG may by viewed as a pass band filter as well. To illustrate this idea let us look closer at Fig. 4-11. Spectrum characteristic between two dips, i.e. around $\lambda: 1550-1555 \mathrm{~nm}$ varies from complete transmission (Fig. 4-11a) to complete stop (Fig. 4-11d) and then to complete transmission again. So, from Fig. 4-11d to Fig. 4-1 la the LPG is acting as a pass band filter in the range of wavelengths $1550-1555 \mathrm{~nm}$. To improve the properties of a pass band filter we can make the two dips slightly wider by chirping the LPG. In principle, the width of the band pass and its position can be made equivalent to the fluorescent band of an erbium-doped amplifier.

4. We have found the limiting value of coupling coefficient, $\kappa_{\lim }$ which guaranties full ONOFF switching by $n_{r}$-modulation. Unfortunately, the length of the device, $L_{g}$ corresponding to $\kappa_{\lim }$ is too large for practical applications, $\sim 2.194 \mathrm{~m}$. Possible approaches to make the device a compact:

- spiral configuration

- use the electro-optic effect to increase the modulated value $\Delta n_{r}$

- use the thermo-optic effect to increase the modulated value $\Delta n_{r}$ In [26] widely tunable long period gratings in fiber, with an additional ultra thin cladding layer, was analyzed. They have found that the notch filter can be tuned over the range $\Delta \lambda=225 \mathrm{~nm}$ by varying the refractive index from 1.43 to 1.33 , so $\Delta n=10^{-2}$. If we estimate the relative range per refractive index change it is: $\frac{\Delta \lambda}{\Delta n}=225 \times 10^{2} \mathrm{~nm}$. In our example in Fig. 4-11a-e the filter is tuned from about 1542 to $1550 \mathrm{~nm}$ by roughly $\Delta n \approx 10^{-6}$ index change, so the relative tenability is much larger: $\frac{\Delta \lambda}{\Delta n}=8 \times 10^{6} \mathrm{~nm}$. 
An increasing value $\Delta n_{r}$ leads to a larger separation between phase matching curves, therefore we can increase value $\kappa_{\lim }$ and reduce length of the device $L_{g}$. In this case the device can be made compact and applicable for integrated optics applications. The geometry of a planar waveguide allows to build an electro-optic element into it, in contrast to the optical fiber.

Alternatively, we can use higher dopant concentration to achieve a larger nonlinear effect. If we apply in our calculation a more optimistic value of enhancement factor given in chapter 1 , $\sim 140000$, then the refractive index modulation due to light intensity can be increased from $10^{-6}$ to $10^{-5}$ and even to $\sim 10^{-4}$. This leads to a significant reduction of the length without having an additional element.

\section{Approximations made.}

in all calculations we have used one dimensional geometry $(1 \mathrm{D}$, slab), however the real structure is two dimensional. Conversion to the $2 \mathrm{D}$ case is straightforward by using the effective refractive index method, [46].

- Modal indices obtained with numerical simulation take into account the dispersion due to waveguide $D_{W}$, by imposing boundary conditions of the structure, and material dispersion $D_{M}$, by introducing a function for the refractive index vs. wavelength $(n(\lambda)$ rather than a fixed number $n$ ) for each layer. The presence of the LPG was accounted by a phase matching condition. This approach however does not 'see' the dispersion of the grating itself $D_{L P G}$. One way to include $D_{L P G}$ in the analysis is to find the dispersion at a resonance wavelength, $D_{L P G}\left(\lambda_{0}\right)$ and multiply it by an 'envelope' function describing the bandwidth of LPG: $D_{L P G}(\lambda)=\operatorname{Env}(\lambda) \cdot D_{L P G}\left(\lambda_{0}\right)$, with $\operatorname{Env}(\lambda)=\frac{1}{\sigma \sqrt{2 \pi}} \exp \left[-\frac{1}{2}\left(\frac{\lambda-\lambda_{0}}{\sigma}\right)^{2}\right]$ 
Then we can use the updated functional dependence of the material refractive index for more accurate numerical simulation: $n(\lambda)_{n e w} \approx n(\lambda)_{\text {old }}+\frac{1}{2 !} D_{L P G}(\lambda) \cdot\left(\lambda-\lambda_{0}\right)^{2}$. In the last expression we have assumed that the dispersion is due to the LPG and the dispersion due to the material can be summed together. For justification of such assumption (total effective dispersion is roughly the sum of its component) see [24]. Intuitively, we can argue that if dispersion due to fiber can be compensated by dispersion of the grating, then total effective dispersion is the sum of both.

Another way to account for LPG dispersion is to apply perturbation techniques. To do this the modal indices of a slab were found, then a periodic variation of the refractive index was introduced and treated as a perturbation. Apply standard perturbation theory and find new modal indices. They will 'see' LPG dispersion.

In our model the nonlinear effects are not built in the underlying waveguide equations. A more accurate way would be to write down nonlinear couple mode equations between appropriate modes with intensity dependent refractive index in the core region and solve them numerically. Then final results, i.e. transmission characteristics, would include nonlinear effects more accurately.

- $\quad$ losses are not included, however they can substantially modify the LPG spectrum. The presence of localized losses in the cladding region of the fiber and their effect on transmission characteristics were analyzed in [47]. It was found that there is an optimal loss factor which makes transmission characteristics wider and sidelobe-free. The important point is that the length which provides $100 \%$ power transfer from the core mode to the cladding, is larger than in the lossless case. Including loss in the accurate model is crucial. Another interesting feature resulting in inclusion of loss and gain is the possibility of a break down of time-reversal symmetry and achievement of unidirectional optical mode interference and coupling, [48]. This concept has been used to design a ring resonator with a grating-assisted coupler which can trap the light, i.e. potential optical memory cell (or optical buffer) [41]. 


\section{Appendix A}

\section{Lemma}

In reference [49] Lemma was proven that propagation constant is monotonic function of the thickness of $i^{\text {th }}$ layer in a multilayer slab. Similar arguments can be applied when free parameter is refractive index of the layer rather than its thickness.

\section{Lemma: propagation constant (or modal effective index) is monotonic function of $\mathbf{n}_{\mathbf{r}}$ (refractive index of the second channel)}

We evaluate propagation constant with the help of perturbation theory. Assume, for the core refractive index $n_{r 0}$ propagation constant $\beta_{0}$ is known (core is defined as a second channel in a five-layer slab). Now the value $n_{r 0}$ has been changed by small amount $\delta n$ :

$n_{r 1}=n_{r 0}+\delta n$, so small that the first order perturbation theory is valid. Then new value of propagation constant evaluated as:

$$
\beta_{1}^{2} \approx \beta_{0}^{2}+\left\langle\psi_{0}|V| \psi_{0}\right\rangle=\beta_{0}^{2}+\left(n_{r 1}^{2}-n_{r 0}^{2}\right) \int_{d}^{p} \psi_{0}^{*} \psi_{0} \cdot d x=\beta_{0}^{2}+\left(n_{r 1}^{2}-n_{r 0}^{2}\right) \cdot \int_{d}^{p}\left|\psi_{0}\right|^{2} \cdot d x
$$

The first term, $\beta_{0}^{2}$ does not depend on $n_{r 1}$. In the second term the integral is always positive $\left(\int_{d}^{p}\left|\psi_{0}\right|^{2} \cdot d x>0\right)$ and does not depend on $n_{r 1}$ either. Therefore we can conclude, if $n_{r}$ is nondecreasing $\left(n_{r 1} \geq n_{r 0}\right)$, then the propagation constant is also non-decreasing, $\beta_{1} \geq \beta_{0}$. And vise versa, if $n_{r}$ is non-increasing $\left(n_{r 1} \leq n_{r 0}\right)$, then the propagation constant is also nonincreasing, $\beta_{1} \leq \beta_{0}$. The function $\beta\left(n_{r}\right)$ is monotonic, it does not have local minima, maxima points for which $\frac{d \beta}{d n_{r}}=0$. 
Whenever perturbation theory (PT) is valid, the result from Lemma is also valid. PT is not valid when perturbation is strong enough such that, perturbed propagation constant significantly different from unperturbed one. In this case we can apply the same perturbation in many steps rather than in one single jump. For example, suppose increment from $n_{r 0}$ to $n_{r 1}$ will change propagation constant by $100 \%$ which is a big number and PT can not handle it. We divide this increment in 100 steps and we expect that the propagation constant will change at each step no more than let's say $2 \%$. Then at each step PT can be applied, therefore at each step the result of the Lemma is valid, and we can say that over the range from $n_{r 0}$ to $n_{r 1}$ propagation constant is monotonic (has no local minima and maxima). Normally we do not know in advance by how much propagation constant will change at the increment from $n_{r 0}$ to $n_{r 1}$, however, in principle, it is always possible to make each step is so small that at each step the propagation constant varies infinitesimally. This last conclusion is actually based on an intuitive assumption, that the propagation constant is a physical quantity that varies continuously with the refractive index of the second channel and should not have steplike jumps or other type of discontinuities. 


\section{Appendix B}

\section{Perturbation theory for stationary states. (Rayleigh- Schrödinger perturbation theory)}

Perturbation theory for stationary states is treated in many textbooks on quantum mechanics, see for example [50], [51], [52].

Notations:

$H_{0} \quad$ Hamiltonian operator for unperturbed system

$\lambda V \quad$ perturbation potential where $\lambda \leq 1$

$\psi_{0} \quad$ wavefunction (solution of the wave equation) for unperturbed system

$\psi \quad$ wavefunction for perturbed system

$E_{0} \quad$ energy eigenvalue for unperturbed system

$E \quad$ energy eigenvalue for perturbed system

$\Delta E \quad$ energy shift for perturbed system, $\Delta E=E-E_{0}$

$P \quad$ projection operator: $P|\psi\rangle=\left|\psi_{0}\right\rangle$

$Q \quad$ operator complement to $P: Q=1-P$. It can also be defined as an operator which projects $|\psi\rangle$ on a state orthogonal to $\left|\psi_{0}\right\rangle:\left\langle\psi_{0}|Q| \psi\right\rangle=0$

For unperturbed and perturbed system we can write Schrödinger equations as follows:

$H_{0}\left|\psi_{0}\right\rangle=E_{0}\left|\psi_{0}\right\rangle$

$\left(H_{0}+\lambda V\right)|\psi\rangle=E|\psi\rangle$

Subtract (B-1) from (B-2):

$\left(H_{0}+\lambda V\right)|\psi\rangle-H_{0}\left|\psi_{0}\right\rangle=E|\psi\rangle-E_{0}\left|\psi_{0}\right\rangle$

Add term $E_{0}|\psi\rangle$ to LHS and RHS: 


$$
\begin{aligned}
& H_{0}|\psi\rangle+\lambda V|\psi\rangle-H_{0}\left|\psi_{0}\right\rangle+E_{0}|\psi\rangle=E|\psi\rangle-E_{0}\left|\psi_{0}\right\rangle+E_{0}|\psi\rangle \\
& H_{0}\left(|\psi\rangle-\left|\psi_{0}\right\rangle\right)+\lambda V|\psi\rangle+E_{0}|\psi\rangle=E|\psi\rangle+E_{0}\left(|\psi\rangle-\left|\psi_{0}\right\rangle\right) \\
& \lambda V|\psi\rangle+\left(E_{0}-E\right)|\psi\rangle=\left(E_{0}-H_{0}\right)\left(|\psi\rangle-\left|\psi_{0}\right\rangle\right) \\
& (\lambda V-\Delta E)|\psi\rangle=\left(E_{0}-H_{0}\right)\left(|\psi\rangle-\left|\psi_{0}\right\rangle\right)
\end{aligned}
$$

In the last equation the RHS is orthogonal to $\left|\psi_{0}\right\rangle$, to verify this we multiply RHS from the left by $\left\langle\psi_{0}\right|$ :

$$
\begin{aligned}
& \left\langle\psi _ { 0 } \left|\left(E_{0}-H_{0}\right)\left(|\psi\rangle-\left|\psi_{0}\right\rangle\right)=\left\langle\psi_{0} \| E_{0} \psi\right\rangle-\left\langle\psi_{0} \| E_{0} \psi_{0}\right\rangle-\left\langle\psi_{0} \| H_{0} \psi\right\rangle+\left\langle\psi_{0} \| H_{0} \psi_{0}\right\rangle=\right.\right. \\
& =\left\langle\psi_{0} \| E_{0} \psi\right\rangle-E_{0}\left\langle\psi_{0} \|_{0} \psi_{0}\right\rangle-\left\langle\psi_{0} \| H_{0} \psi\right\rangle+E_{0}\left\langle\psi_{0} \| \psi_{0}\right\rangle=\left\langle\psi_{0} \| E_{0} \psi\right\rangle-\left\langle\psi_{0} \| H_{0} \psi\right\rangle
\end{aligned}
$$

Operator $H_{0}$ has the property: $\left\langle\psi_{0} \| H_{0} \psi\right\rangle=\left\langle H_{0} \psi_{0} \| \psi\right\rangle$, i.e. $H_{0}$ is Hermitian operator, therefore:

$$
\left\langle\psi_{0} \| E_{0} \psi\right\rangle-\left\langle\psi_{0} \| H_{0} \psi\right\rangle=E_{0}\left\langle\psi_{0} \| \psi\right\rangle-\left\langle H_{0} \psi_{0} \| \psi\right\rangle=E_{0}\left\langle\psi_{0} \| \psi\right\rangle-E_{0}\left\langle\psi_{0} \| \psi\right\rangle=0
$$

So, RHS of (B-7) is orthogonal to $\left|\psi_{0}\right\rangle$, then LHS of (B-7) is also orthogonal to $\left|\psi_{0}\right\rangle$ :

$$
\left\langle\psi_{0}|(\lambda V|\psi\rangle-\Delta E|\psi\rangle)=0\right.
$$

From (B-10) energy shift of perturbed system can be found:

$$
\Delta E=\lambda \frac{\left\langle\psi_{0}|V| \psi\right\rangle}{\left\langle\psi_{0} \| \psi\right\rangle}
$$

If perturbed wavefunction $|\psi\rangle$ normalized in such a way that $\left\langle\psi_{0} \| \psi\right\rangle=1$, then

$$
E=E_{0}+\lambda\left\langle\psi_{0}|V| \psi\right\rangle
$$

So far we have not made any approximation, therefore expression (B-12) for new (perturbed) energy level is exact. (B-12) also tells that if we know new wavefunction of the system $|\psi\rangle$ approximately, let's say with the terms up to the order $\lambda^{n-1}$, then energy level can be determined with the terms up to the order $\lambda^{n}$. Now we want to estimate first order of $E$, 
therefore zero order of $|\psi\rangle$ is needed. We have shown, that RHS of (B-7) is orthogonal to $\left|\psi_{0}\right\rangle$ and so the LHS. Therefore, operator $Q$ will not change LHS. ( $Q$ is a projector on a state orthogonal to $\left|\psi_{0}\right\rangle$, if a given state is already orthogonal to $\left|\psi_{0}\right\rangle$, then applying $Q$ to it does not modify this state, schematic diagram for operators $P$ and $Q$ is shown in Fig. B-1):

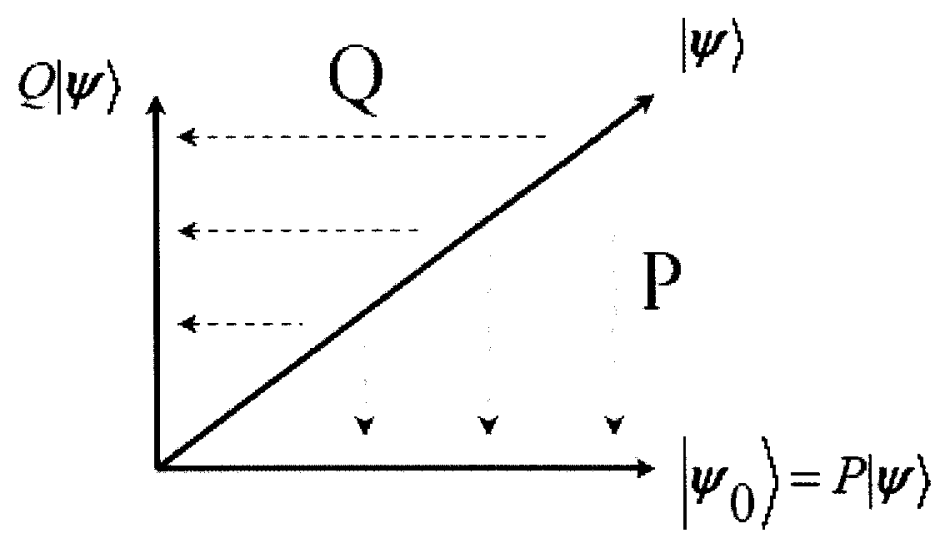

Fig. B-1. Diagram illustrates action of projection operator $P$ and operator $Q$ on a wavefunction $|\psi\rangle$.

$(\lambda V-\Delta E)|\psi\rangle=Q(\lambda V-\Delta E)|\psi\rangle$

State $\left(|\psi\rangle-\left|\psi_{0}\right\rangle\right)$ can be written as $Q|\psi\rangle$ :

$\left(|\psi\rangle-\left|\psi_{0}\right\rangle\right)=|\psi\rangle-P|\psi\rangle=(1-P)|\psi\rangle=Q|\psi\rangle$

Then (B-7) becomes:

$Q(\lambda V-\Delta E)|\psi\rangle=\left(E_{0}-H_{0}\right) Q|\psi\rangle$

from which we can find zero order $|\psi\rangle$ : 
$Q|\psi\rangle=\frac{1}{E_{0}-H_{0}} Q(\lambda V-\Delta E)|\psi\rangle$

$|\psi\rangle=\left|\psi_{0}\right\rangle+\frac{1}{E_{0}-H_{0}} Q(\lambda V-\Delta E)|\psi\rangle$

Zero order $|\psi\rangle$ is just $\left|\psi_{0}\right\rangle$, then first order energy level is:

$E=E_{0}+\lambda\left\langle\psi_{0}|V| \psi_{0}\right\rangle$

Energy level $E$ and modified wavefunction $|\psi\rangle$ can be expanded as a power series in terms of $\lambda$, in this case we can use extreme value for $\lambda: \lambda=1$. ( $\lambda$ is defined as a ratio of perturbed potential $\lambda V$ to some potential $V, \lambda=\frac{\lambda V}{V} \leq 1$.). Potential $V$ should be small enough compare to $H_{0}$, so that the perturbation does not modify system significantly. However how small we do not know, i.e. we do not have quantitative estimation $\frac{V}{H_{0}}$ for which obtained values of $E$ and $|\psi\rangle$ are reasonably close to the true values of $E$ and $|\psi\rangle$. Another words, ideally we want to see $E$ and $|\psi\rangle$ as a power series expansions in terms of parameter $\gamma, \gamma=\frac{V}{H_{0}}$, rather than $\lambda$. This is limitations of perturbation theory.

Each new term in power series expansion is harder to calculate and it becomes too complex, so we will use only first order term but still apply extreme value for $\lambda$, i.e. $\lambda=1$, assuming that our perturbation $V$ is small enough compare to $H_{0}$, so that an estimated first order value of $E$ is reasonably close to the true value of energy level.

$$
\begin{aligned}
& E^{(1)}=E_{0}+\left\langle\psi_{0}|V| \psi_{0}\right\rangle \\
& E_{0}=\left\langle\psi_{0}\left|H_{0}\right| \psi_{0}\right\rangle \\
& E^{(1)}=\left\langle\psi_{0}\left|H_{0}\right| \psi_{0}\right\rangle+\left\langle\psi_{0}|V| \psi_{0}\right\rangle=\left\langle\psi_{0}|H| \psi_{0}\right\rangle=\left\langle\psi_{0}\left(E_{0}\right)|H| \psi_{0}\left(E_{0}\right)\right\rangle \\
& E^{(1)}=\left\langle\psi_{0}\left(E_{0}\right)|H| \psi_{0}\left(E_{0}\right)\right\rangle
\end{aligned}
$$


$E=\langle\psi(E)|H| \psi(E)\rangle$

Expression (B-23) is exact value for perturbed energy level, it requires both modified Hamiltonian and modified wavefunction which depends on new (perturbed) energy level. Expression (B-22) is a first order approximation for perturbed energy level obtained from Rayleigh- Schrödinger perturbation theory. To calculate $E^{(1)}$ we need to know perturbed Hamiltonian $H$ and old unmodified wavefunction which depends on unmodified energy level, $\left|\psi_{0}\left(E_{0}\right)\right\rangle$. It does not require root finding, it is a direct calculation. (B-22) is used when modified wavefunction is not known, or difficult to evaluate. 


\section{Appendix C}

\section{Characteristic equation for symmetric three-layer slab waveguide}

Helmholtz wave equation slab configuration has a form:

$$
\begin{aligned}
& \frac{d^{2}}{d x^{2}} e_{y}+\left(k^{2} n^{2}-\beta^{2}\right) e_{y}=0 \\
& h_{z}=\frac{j}{\omega \mu} e_{y}
\end{aligned}
$$

Its solution depends on relation $k n$ and $\beta$. For guided modes in the slab this relation is:

$k n_{b} \leq \beta \leq k n_{c}$

Define effective refractive index (modal refractive index) as:

$\beta=k n_{\text {eff }}$

Introduce new variables:

$$
\begin{aligned}
& u_{c}^{2}=k^{2} n_{c}^{2}-\beta^{2}=k^{2}\left(n_{c}^{2}-n_{e f f}^{2}\right) \geq 0 \\
& u_{b}^{2}=\beta^{2}-k^{2} n_{b}^{2}=k^{2}\left(n_{e f f}^{2}-n_{b}^{2}\right) \geq 0
\end{aligned}
$$

Then, in each region of the slab solution is:

$$
\begin{aligned}
& x<0 \quad e_{y}=A_{1} \exp \left[u_{b} x\right] \\
& h_{z}=\frac{j}{\omega \mu} u_{b} A_{1} \exp \left[u_{b} x\right] \\
& 0<x<\mathrm{v} \quad e_{y}=A_{2} \sin \left(u_{c} x\right)+B_{2} \cos \left(u_{c} x\right) \\
& h_{z}=\frac{j}{\omega \mu} u_{c}\left(A_{2} \cos \left(u_{c} x\right)-B_{2} \sin \left(u_{c} x\right)\right) \\
& x>\mathrm{v} \quad e_{y}=A_{3} \exp \left[-u_{b} x\right] \\
& h_{z}=\frac{j}{\omega \mu}(-1) u_{b} A_{3} \exp \left[-u_{b} x\right]
\end{aligned}
$$


At the each interface of adjacent layers tangential field components are continuous:

$$
\begin{array}{ll}
x=0 & e_{y}: A_{1}=B_{2} \\
& h_{z}: u_{b} A_{1}=u_{c} A_{2} \\
x=\mathrm{v} & e_{y}: A_{2} \sin \left(u_{c} \mathrm{v}\right)+B_{2} \cos \left(u_{c} \mathrm{v}\right)=A_{3} \exp \left[-u_{b} \mathrm{v}\right] \\
& h_{z}: u_{c}\left(A_{2} \cos \left(u_{c} \mathrm{v}\right)-B_{2} \sin \left(u_{c} \mathrm{v}\right)\right)=-u_{b} A_{3} \exp \left[-u_{b} \mathrm{v}\right]
\end{array}
$$

Eliminating $A_{1}$ from (C-13), (C-14) gives expression for $B_{2}$ :

$$
B_{2}=\frac{u_{c}}{u_{b}} A_{2}
$$

Use above result for $B_{2}$ and find $A_{3}$ from (C-15):

$$
A_{3}=\exp \left[u_{b} \mathrm{v}\right] A_{2}\left(\sin \left(u_{c} \mathrm{v}\right)+\frac{u_{c}}{u_{b}} \cos \left(u_{c} \mathrm{v}\right)\right)
$$

Use result for $B_{2}$ and find $A_{3}$ from (C-16):

$$
A_{3}=-\exp \left[u_{b} \mathrm{v}\right] A_{2} \frac{u_{c}}{u_{b}}\left(\cos \left(u_{c} \mathrm{v}\right)-\frac{u_{c}}{u_{b}} \sin \left(u_{c} \mathrm{v}\right)\right)
$$

From (C-18), (C-19) we obtain characteristic equation for propagation constant $\beta$ (or, for modal refractive index $\left.n_{\text {eff }}\right)$ :

$$
\sin \left(u_{c} \mathrm{v}\right)+\frac{u_{c}}{u_{b}} \cos \left(u_{c} \mathrm{v}\right)=-\frac{u_{c}}{u_{b}}\left(\cos \left(u_{c} \mathrm{v}\right)-\frac{u_{c}}{u_{b}} \sin \left(u_{c} \mathrm{v}\right)\right)
$$

Multiply by $\left(u_{b}\right)^{2}$ and rearrange the terms:

$$
\begin{aligned}
& \left(u_{c}^{2}-u_{b}^{2}\right) \sin \left(u_{c} \mathrm{v}\right)-2 u_{c} u_{b} \cos \left(u_{c} \mathrm{v}\right)=0 \\
& \left(u_{c}^{2}-u_{b}^{2}\right)^{2}+\left(-2 u_{c} u_{b}\right)^{2}=\left(u_{c}^{2}+u_{b}^{2}\right)^{2} \text { therefore we can introduce parameter } \theta \\
& \left(u_{c}^{2}+u_{b}^{2}\right)\left\{\frac{u_{c}^{2}-u_{b}^{2}}{u_{c}^{2}+u_{b}^{2}} \sin \left(u_{c} \mathrm{v}\right)-\frac{2 u_{c} u_{b}}{u_{c}^{2}+u_{b}^{2}} \cos \left(u_{c} \mathrm{v}\right)\right\}=0 \\
& \sin \theta=\frac{2 u_{c} u_{b}}{u_{c}^{2}+u_{b}^{2}}, \quad \cos \theta=\frac{u_{c}^{2}-u_{b}^{2}}{u_{c}^{2}+u_{b}^{2}}
\end{aligned}
$$


$\cos \theta \sin \left(u_{c} \mathrm{v}\right)-\sin \theta \cos \left(u_{c} \mathrm{v}\right)=0$

Possible solutions are:

1): $\cos \left(u_{c} \mathrm{v}\right)=\cos \theta=\frac{u_{c}^{2}-u_{b}^{2}}{u_{c}^{2}+u_{b}^{2}}$

$$
\sin \left(u_{c} \mathrm{v}\right)=\sin \theta=\frac{2 u_{c} u_{b}}{u_{c}^{2}+u_{b}^{2}}
$$

2): $\cos \left(u_{c} \mathrm{v}\right)=-\cos \theta=-\frac{u_{c}^{2}-u_{b}^{2}}{u_{c}^{2}+u_{b}^{2}}$

$$
\sin \left(u_{c} \mathrm{v}\right)=-\sin \theta=-\frac{2 u_{c} u_{b}}{u_{c}^{2}+u_{b}^{2}}
$$

Introduce new variables $P$ and $Q$ :

$$
\begin{aligned}
& P^{2}=\left(u_{c}^{2}+u_{b}^{2}\right) \mathrm{v}^{2}=k^{2} \mathrm{v}^{2}\left(n_{c}^{2}-n_{b}^{2}\right)=\mathrm{const}\left(n_{\text {eff }}\right) \\
& Q^{2}=u_{c}^{2} \mathrm{v}^{2}=k^{2} \mathrm{v}^{2}\left(n_{c}^{2}-n_{e f f}^{2}\right)
\end{aligned}
$$

Then in terms of $P$ and $Q$ solutions are:

$1): \cos (Q)=\frac{2 Q^{2}-P^{2}}{P^{2}}$

$$
\sin (Q)=\frac{2 Q \sqrt{P^{2}-Q^{2}}}{P^{2}}
$$

2): $\cos (Q)=\frac{P^{2}-2 Q^{2}}{P^{2}}$

$$
\sin (Q)=-\frac{2 Q \sqrt{P^{2}-Q^{2}}}{P^{2}}
$$




\section{Appendix D}

\section{Propagation constant in symmetric three-layer slab waveguide}

Variables $P$ and $Q$ defined as:

$$
\begin{aligned}
& P^{2}=\left(u_{c}^{2}+u_{b}^{2}\right) \mathrm{v}^{2}=k^{2} \mathrm{v}^{2}\left(n_{c}^{2}-n_{b}^{2}\right)=\mathrm{const}\left(n_{\text {eff }}\right) \\
& Q^{2}=u_{c}^{2} \mathrm{v}^{2}=k^{2} \mathrm{v}^{2}\left(n_{c}^{2}-n_{e f f}^{2}\right)
\end{aligned}
$$

Then in terms of $P$ and $Q$ characteristic (eigenvalue) equations are:

1) call this set even modes:

$$
\left\{\begin{array}{c}
\cos (Q)=\frac{2 Q^{2}-P^{2}}{P^{2}} \\
\sin (Q)=\frac{2 Q \sqrt{P^{2}-Q^{2}}}{P^{2}}
\end{array}\right.
$$

2) odd modes:

$$
\left\{\begin{array}{c}
\cos (Q)=\frac{P^{2}-2 Q^{2}}{P^{2}} \\
\sin (Q)=-\frac{2 Q \sqrt{P^{2}-Q^{2}}}{P^{2}}
\end{array}\right.
$$

Even modes.

Maximum positive value of $Q$ should not exceed $P$ :

$$
\begin{aligned}
& \cos (Q)=\frac{2 Q^{2}-P^{2}}{P^{2}} \leq|1| \\
& \Rightarrow-P \leq Q \leq P
\end{aligned}
$$

From graphs $y=\cos (x), y=\frac{2 x^{2}-P^{2}}{P^{2}}$ we see that intersections of two plots happen in each $\pi$-interval $n \pi<x<(n+1) \pi, \quad n=0,1,2 \ldots$ along $x$-axis up until $x=P$. However, common 
solutions with $\sin (x)=\frac{2 x \sqrt{P^{2}-x^{2}}}{P^{2}}$ are only those which satisfy

$2 n \pi<x<(2 n+1) \pi, \quad n=0,1,2, \ldots$, i.e. common solutions are located in every other $\pi$ interval. (see Fig. D-1, 2). 

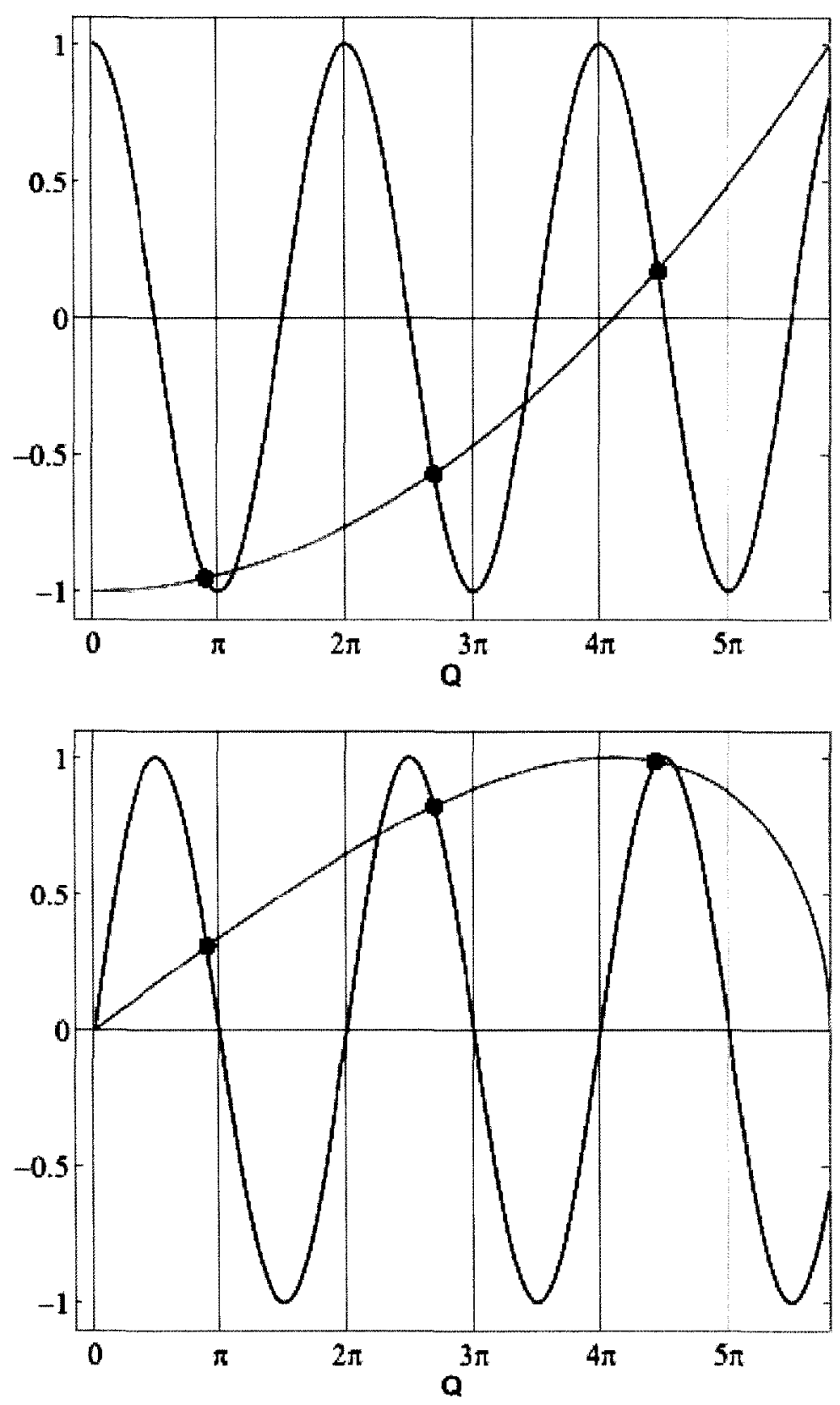

Fig. D-1. Even modes.

top graphs, $y(Q)=\frac{2 Q^{2}-P^{2}}{P^{2}}$ and $y(Q)=\cos (Q)$

bottom graphs, $y(Q)=\frac{2 Q \sqrt{P^{2}-Q^{2}}}{P^{2}}$ and $y(Q)=\sin (Q)$

Proper solutions are marked by black dots. 

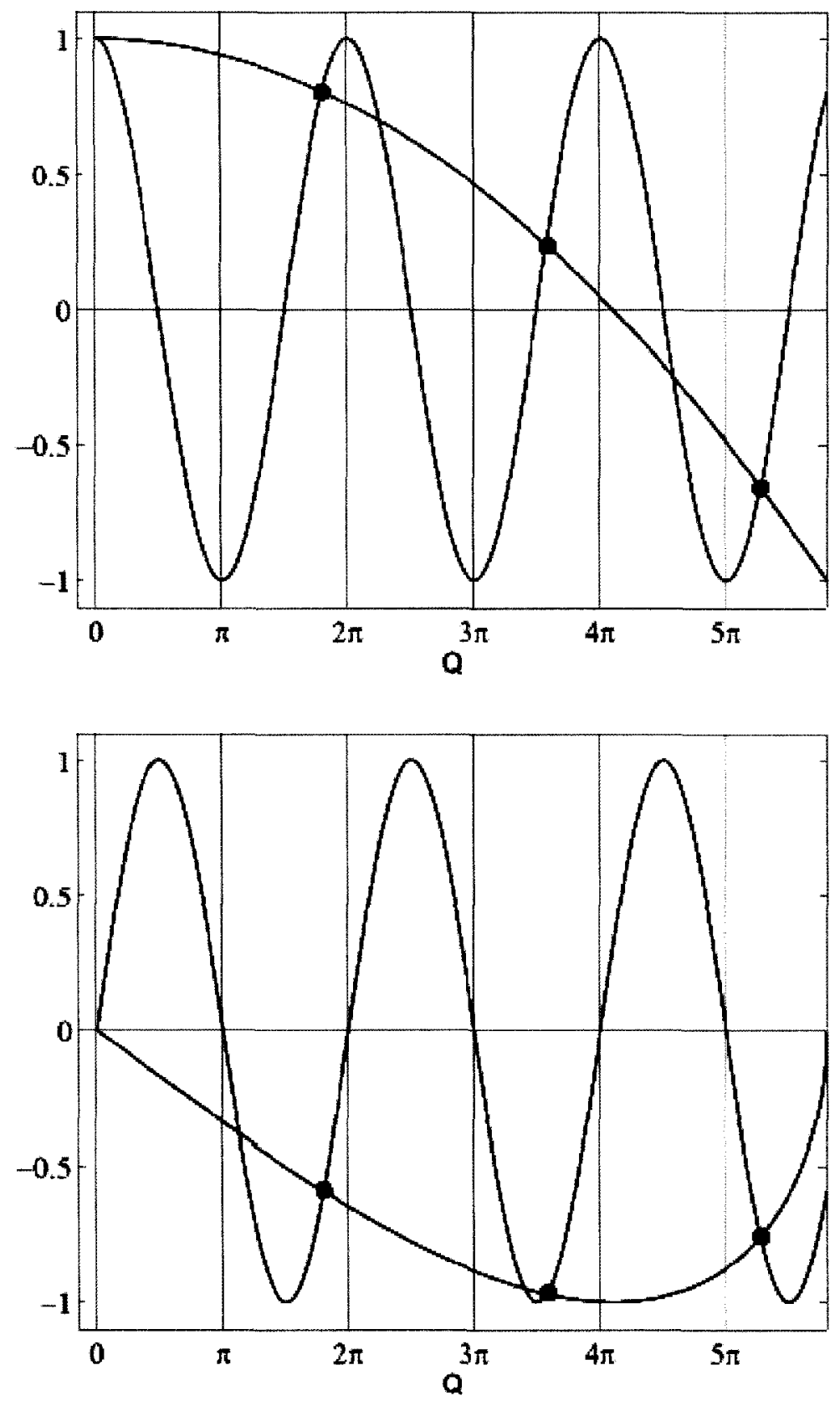

Fig. D-2. Odd modes.

top graphs, $y(Q)=\frac{P^{2}-2 Q^{2}}{P^{2}}$ and $y(Q)=\cos (Q)$

bottom graphs, $y(Q)=-\frac{2 Q \sqrt{P^{2}-Q^{2}}}{P^{2}}$ and $y(Q)=\sin (Q)$

Proper solutions are marked by black dots. 
For odd modes common solutions $\cos (x)=\frac{P^{2}-2 x^{2}}{P^{2}}$ and $\sin (x)=-\frac{2 x \sqrt{P^{2}-x^{2}}}{P^{2}}$ are located in intervals $(2 n+1) \pi<x<(2 n+2) \pi, \quad n=0,1,2, \ldots$ From these observations, total number of modes in symmetric slab waveguide is, $M=$ ceiling $\left(\frac{P}{\pi}\right)$, where function ceiling $(x)$ rounds $x$ to nearest integer greater or equal to $x, M=$ ceiling $\left(\frac{k v \sqrt{n_{c}^{2}-n_{b}^{2}}}{\pi}\right)$. To find approximate analytical solutions we expand $\operatorname{cosine}$ as $\cos (x) \approx 1-\frac{1}{2 !} x^{2}+\frac{1}{4 !} x^{4}$. This expansion works reasonably well in the range of argument $-\frac{\pi}{2} \leq x \leq \frac{\pi}{2}$.

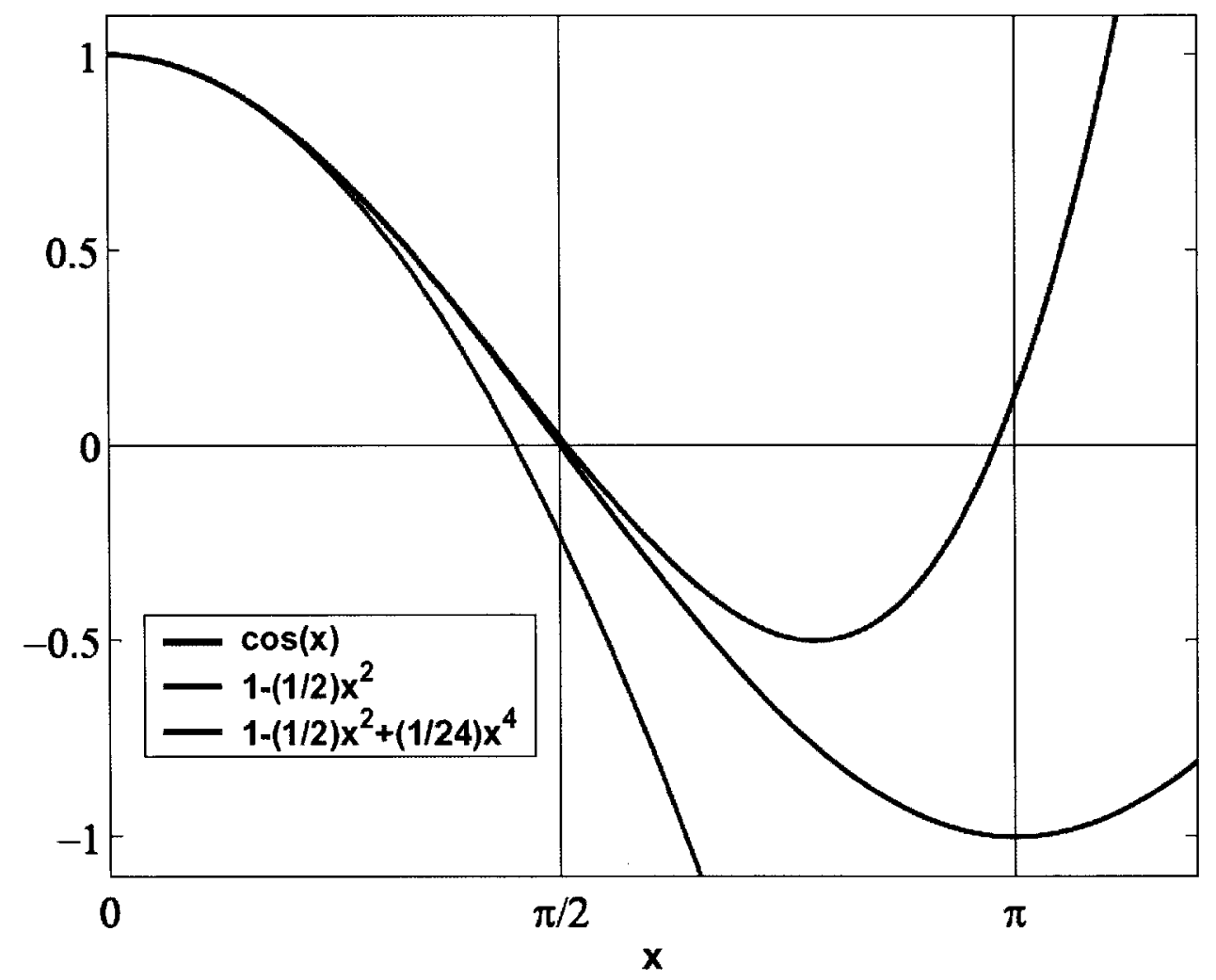

Fig. D-3. Cosine function, its second and forth order expansion. 
In the Fig. D-3 we have shown plots of cos-functions and its 2-order and 4-order expansions. The worst error at the boundary of range $-\frac{\pi}{2} \leq x \leq \frac{\pi}{2}$ is: $\cos \left(\frac{\pi}{2}\right)=0$,

$$
\text { expansion }\left(\frac{\pi}{2}\right) \approx 1-\frac{1}{2 !}\left(\frac{\pi}{2}\right)^{2}+\frac{1}{4 !}\left(\frac{\pi}{2}\right)^{4} \cong 0.01997 \text {. }
$$

The slope of cosine near $\frac{\pi}{2}$ is -1 , therefore about the same absolute error, $\cong 0.01997$ is along $\mathrm{x}$-axis. From this we can estimate the worst error in terms of modal indices:

$Q_{0}=\frac{\pi}{2}$

$Q_{01}=\frac{\pi}{2}+0.01997$

$n_{e f f 0}=\sqrt{n_{c}^{2}-\left(\frac{Q_{0}}{k \mathrm{v}}\right)^{2}}$

$n_{\text {eff } 1}=\sqrt{n_{c}^{2}-\left(\frac{Q_{01}}{k v}\right)^{2}}$

For the example with the following parameters, $\lambda=1.55 \mu \mathrm{m}, v=5 \mu \mathrm{m}, n_{c}=1.52$ we get absolute error in $n_{\text {eff }}: n_{e f f 0}-n_{\text {eff } 01} \cong 5.06 \cdot 10^{-5}$. For higher order modes $\left(Q_{n}=n \frac{\pi}{2}, n>1\right)$ this error is even smaller.

For $x$ outside this range the error between cosine and its 4-order expansion is progressively worse. Therefore, to keep error small we have to expand cosine function close to the root of each mode. This means, we have to expand cosine near $x=0$, then near $x=\pi$, near $x=2 \pi$ and so on up until $x=\pi \cdot\left(\frac{P}{\pi}\right)_{\mathrm{int}}$ 
As seen from the graph (Fig. D-4, 5) for even modes the points of expansion of cosine function should be $x_{0}=\pi, 3 \pi, \ldots(2 n+1) \pi$ whenever $x \leq \frac{P}{\sqrt{2}}$, (value $\frac{P}{\sqrt{2}}$ is the root of function $y=\frac{2 x^{2}-P^{2}}{P^{2}}$ ). And whenever $x \geq \frac{P}{\sqrt{2}}$, expansion points should be $x_{0}=2 n \pi$.

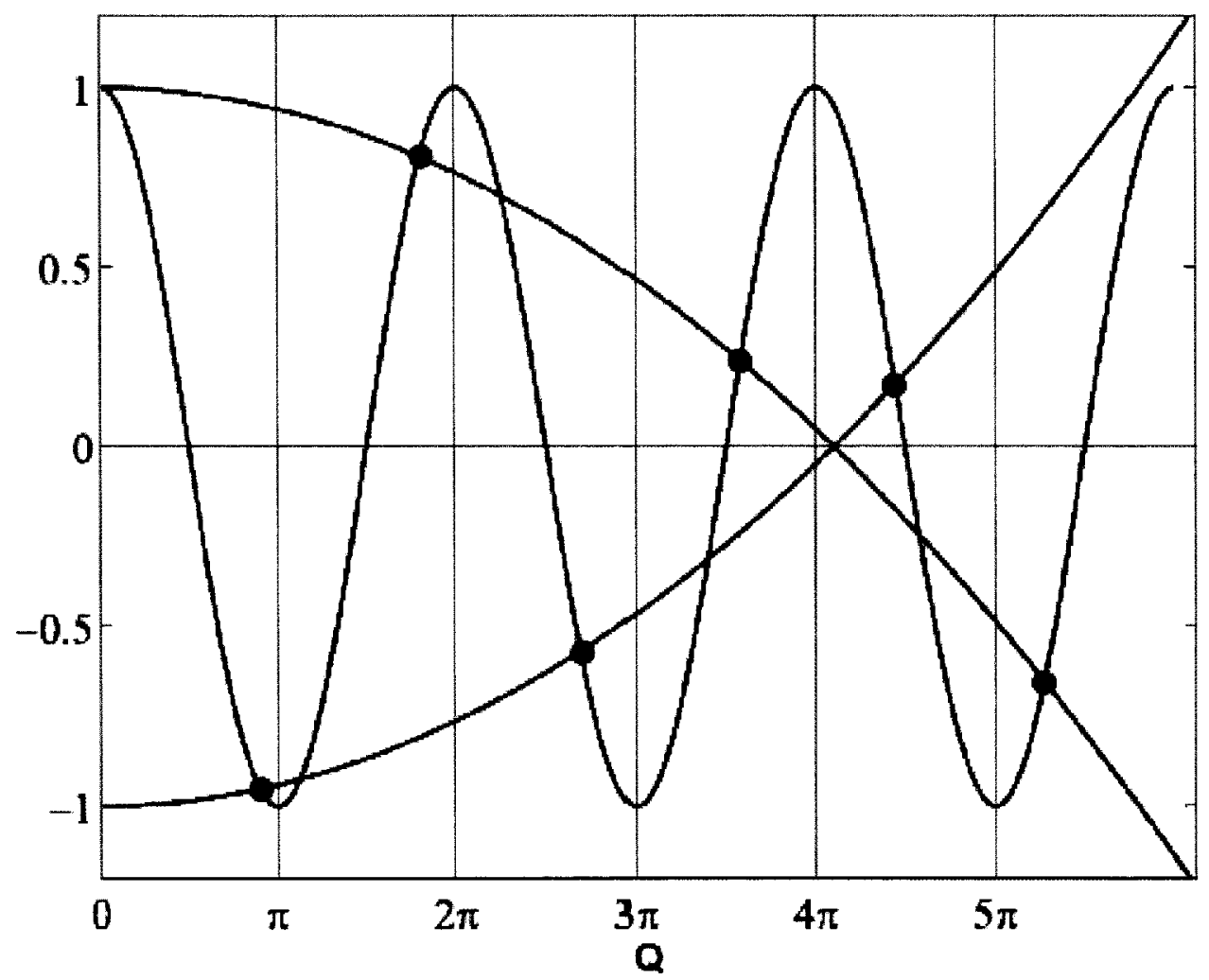

Fig. D-4. Even and odd modes. Graphs $y(Q)=\frac{2 Q^{2}-P^{2}}{P^{2}}, y(Q)=\frac{P^{2}-2 Q^{2}}{P^{2}}$ and $y(Q)=\cos (Q)$. For even modes solutions are on negative slope of cosine, in intervals: $[0.5 \pi, \pi],[2.5 \pi, 3 \pi],[4 \pi, 4.5 \pi]$. For odd modes solutions are on positive slope of cosine, in intervals: $[1.5 \pi, 2 \pi],[3.5 \pi, 4 \pi],[5 \pi, 5.5 \pi]$. 


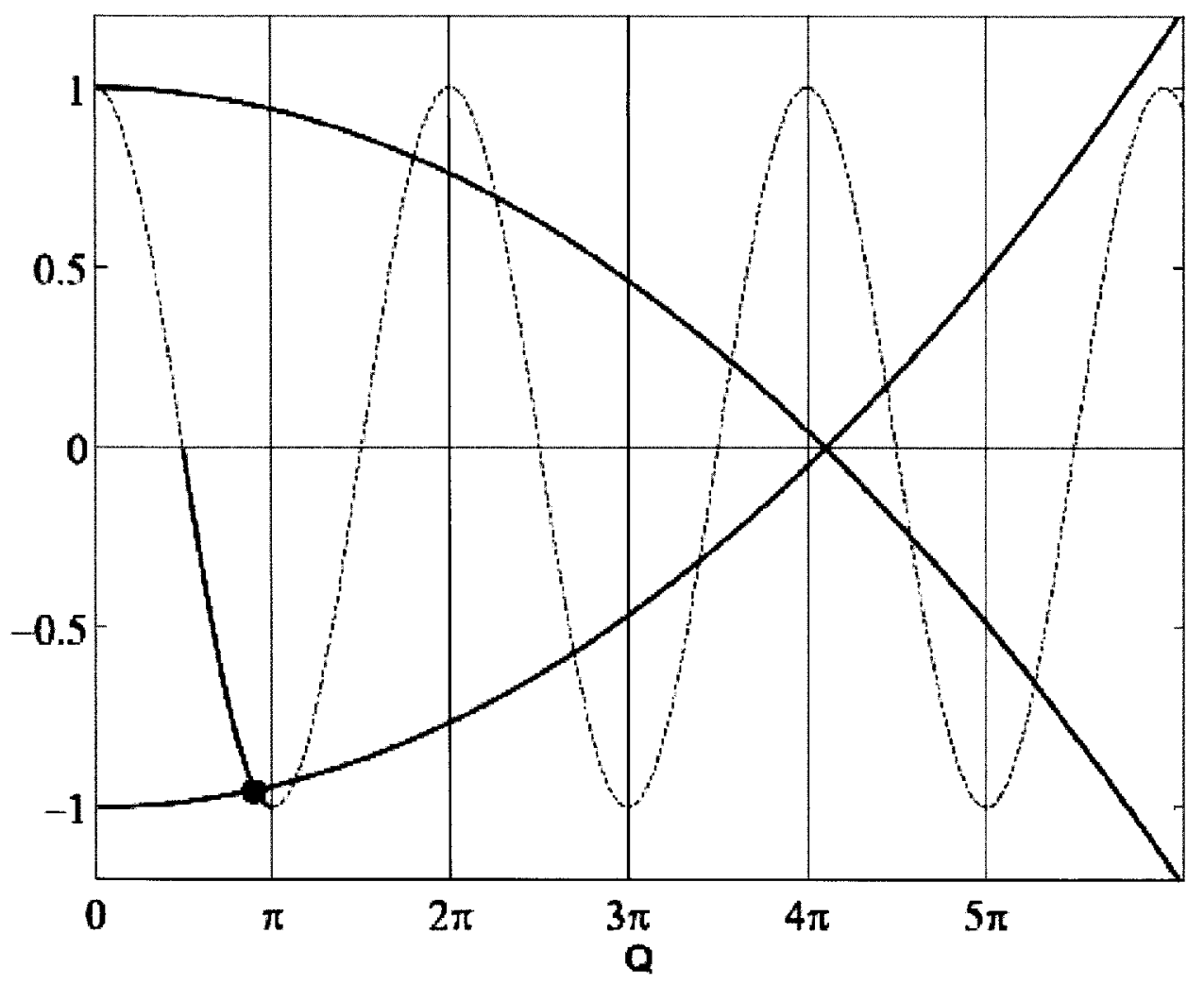

Fig. D-5a. Even mode. Solution is in interval $[0.5 \pi, \pi]$. 


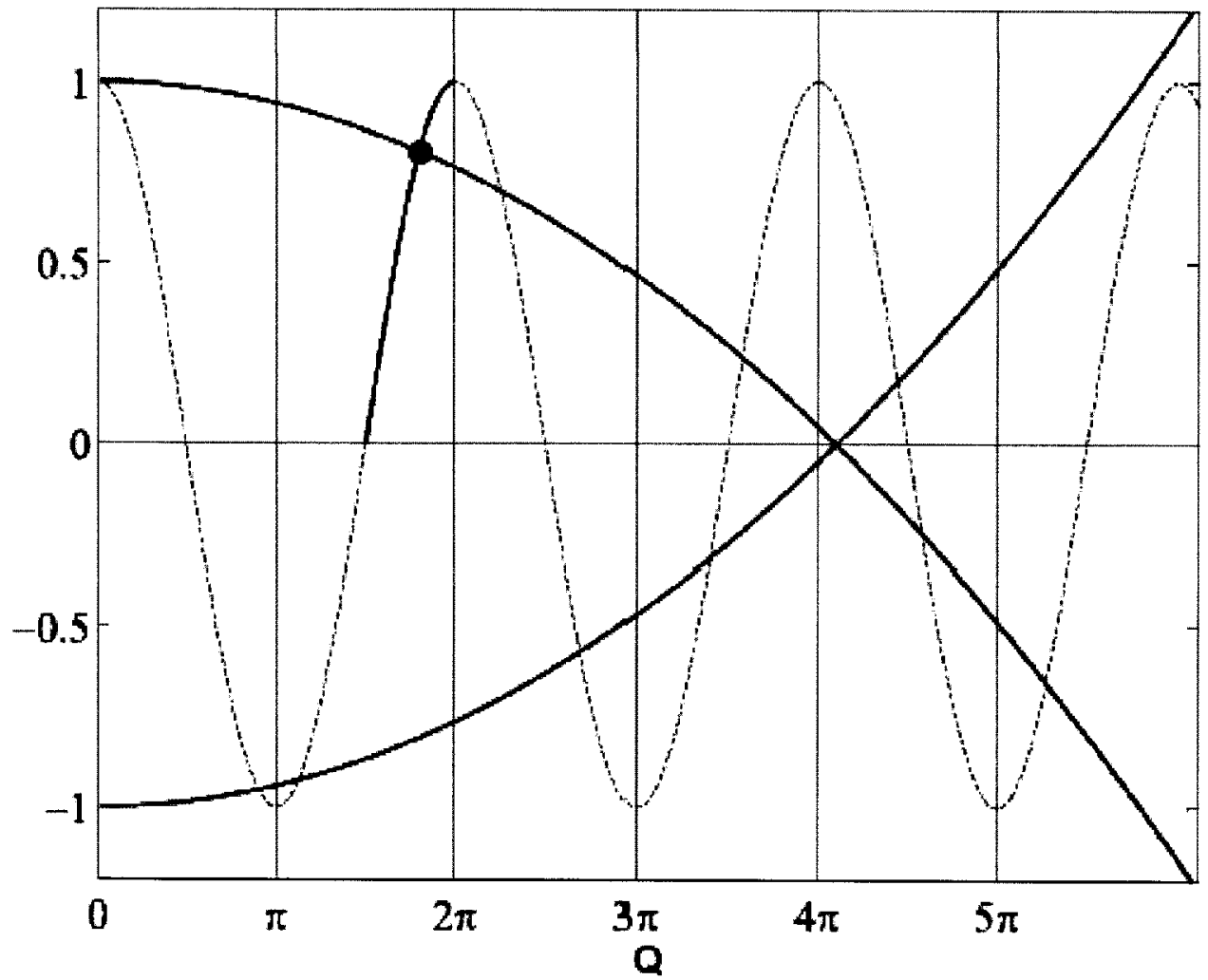

Fig. D-5b. Odd mode. Solution is in interval $[1.5 \pi, 2 \pi]$. 


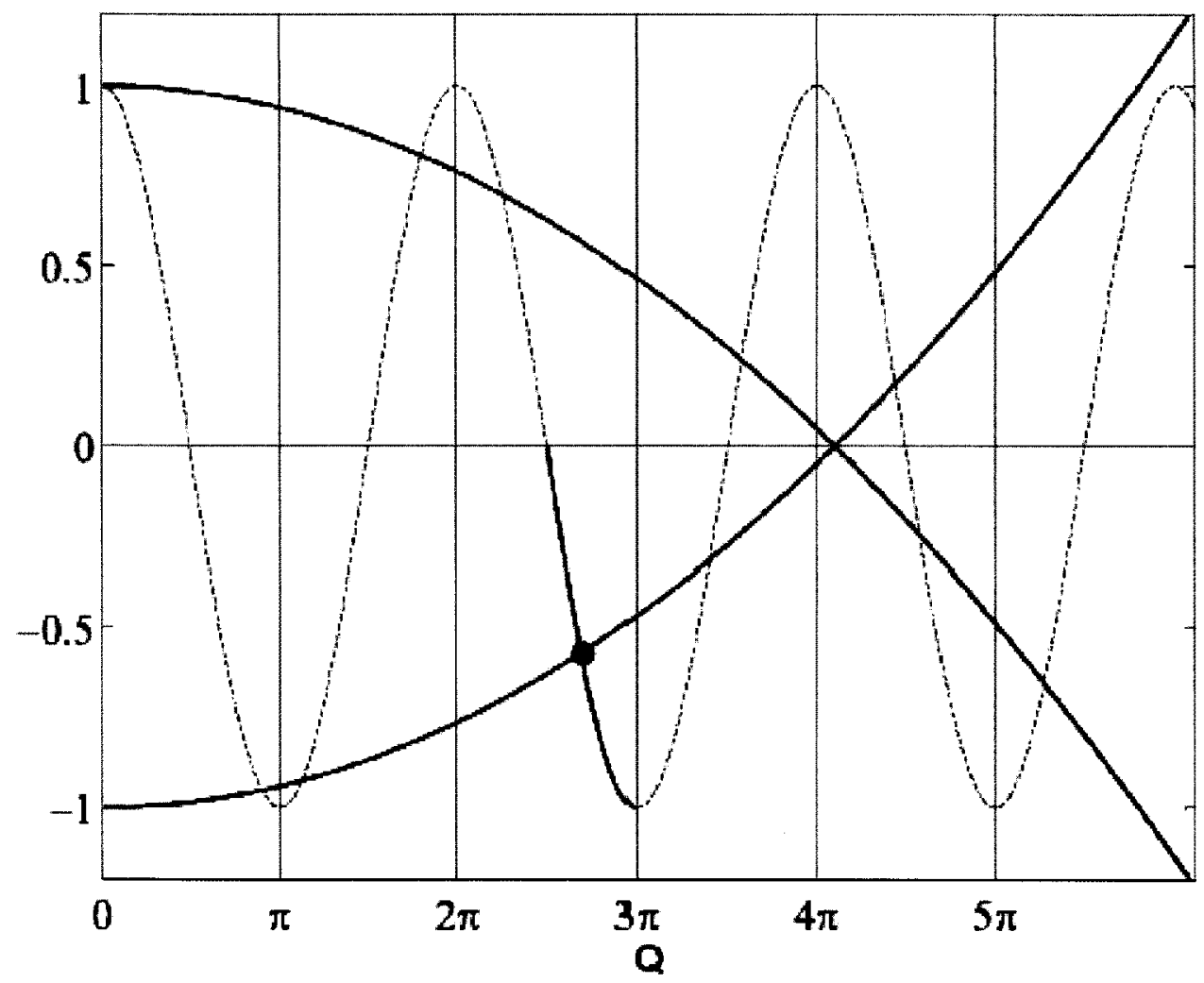

Fig. D-5c. Even mode. Solution is in interval $[2.5 \pi, 3 \pi]$. 


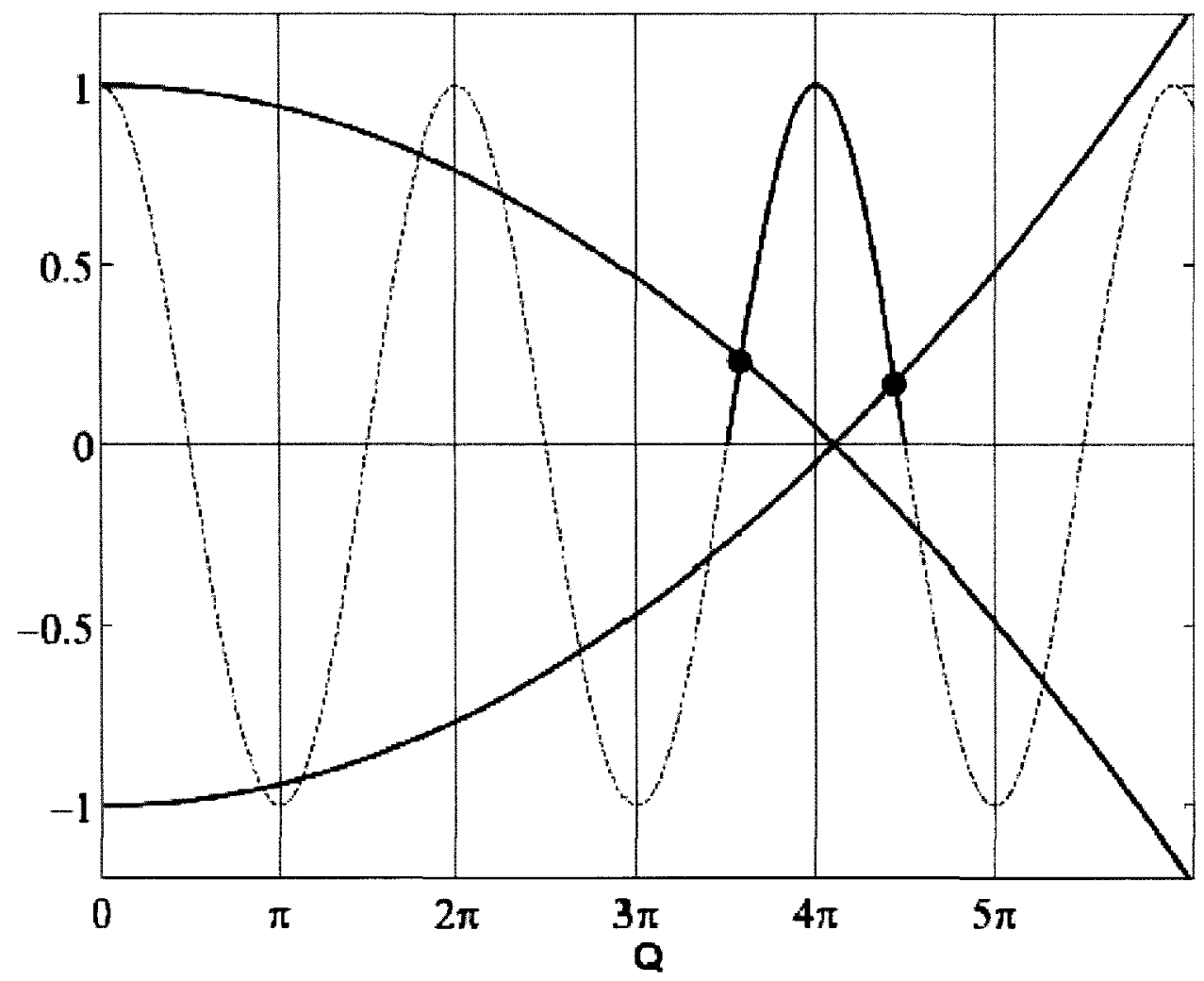

Fig. D-5d. Even mode. Solution is in interval $[4 \pi, 4.5 \pi]$. Odd mode. Solution is in interval $[3.5 \pi, 4 \pi]$. 


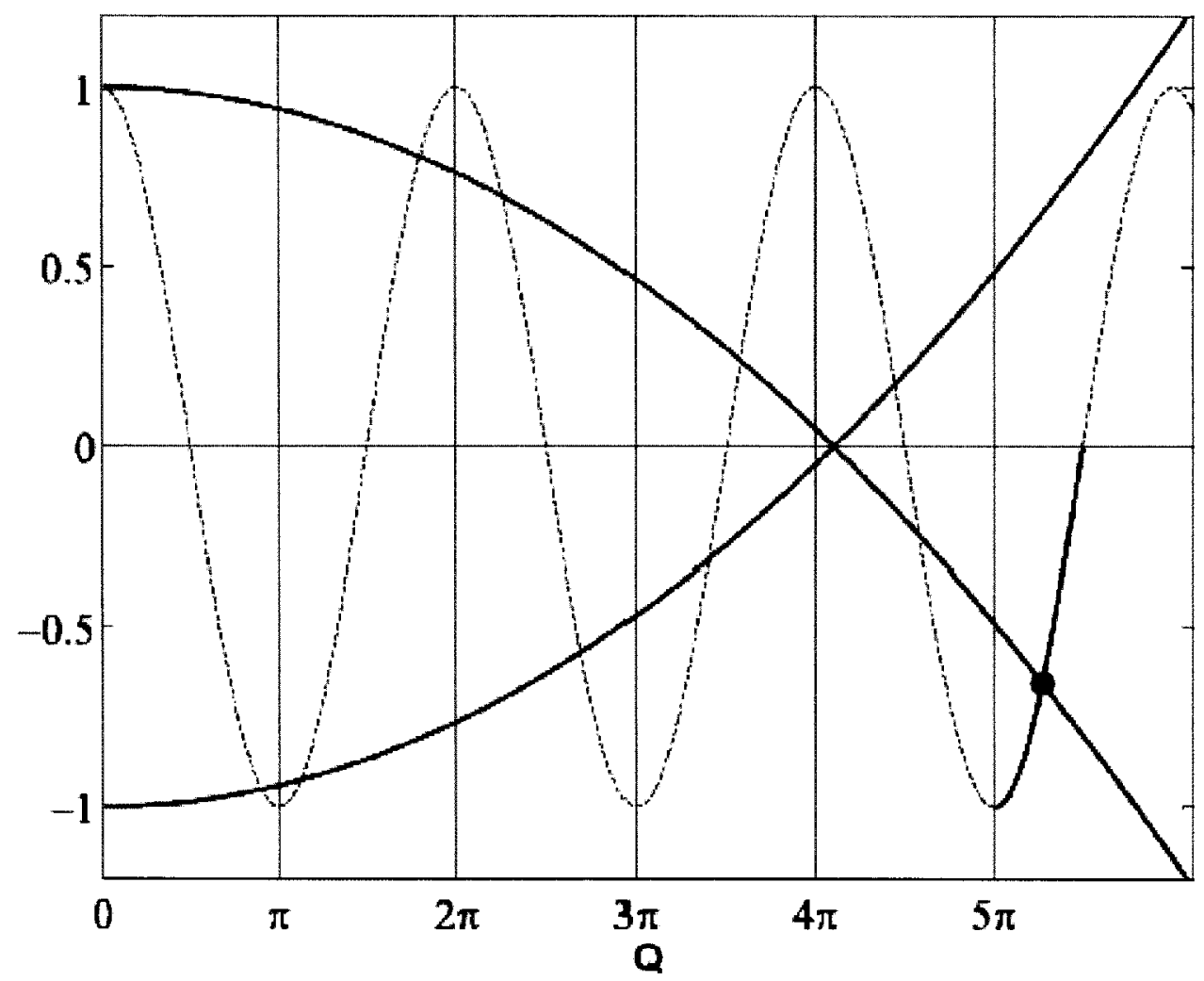

Fig. D-5e. Odd mode. Solution is in interval $[5 \pi, 5.5 \pi]$.

This rule keeps distance between roots and expansion point no more than $\frac{\pi}{2}$. To summarize, for even modes: 


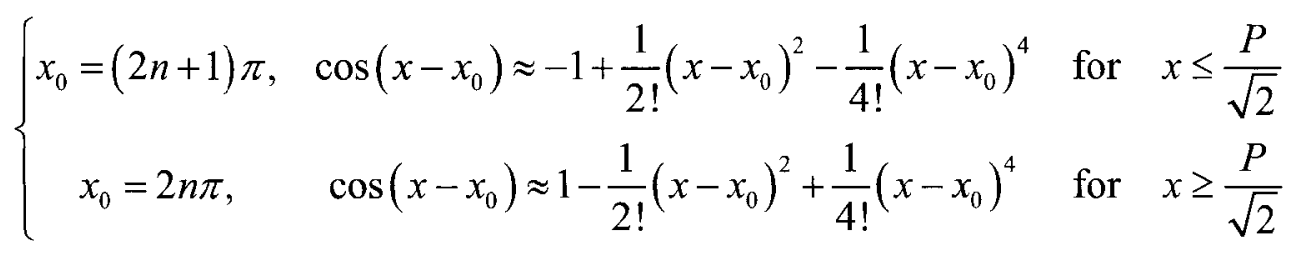

For odd modes expansion points are:

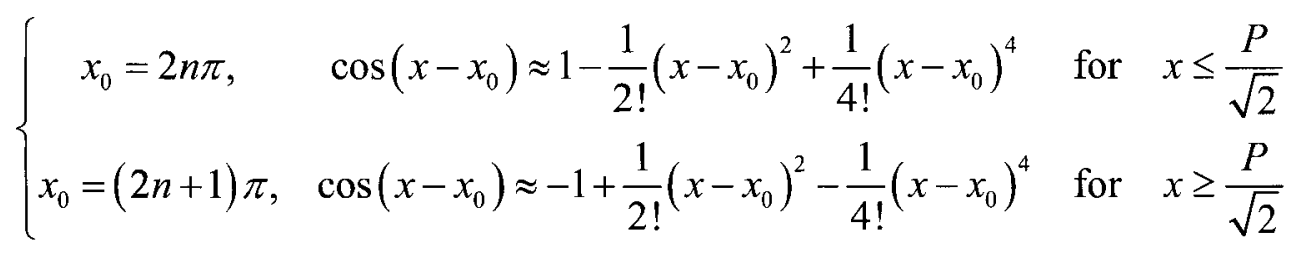

Approximate equations we have to solve are:

\section{even modes}

$-1+\frac{1}{2 !}\left(x-x_{0}\right)^{2}-\frac{1}{4 !}\left(x-x_{0}\right)^{4}=\frac{2 x^{2}-P^{2}}{P^{2}}$

where

$x_{0}=(2 n+1) \pi$

$x_{0}-\frac{\pi}{2} \leq x \leq x_{0} \leq \frac{P}{\sqrt{2}} \overrightarrow{A B}$

and

$1-\frac{1}{2 !}\left(x-x_{0}\right)^{2}+\frac{1}{4 !}\left(x-x_{0}\right)^{4}=\frac{2 x^{2}-P^{2}}{P^{2}}$

where

$x_{0}=2 n \pi$

$\frac{P}{\sqrt{2}} \leq x_{0} \leq x \leq x_{0}+\frac{\pi}{2}$

odd modes 
$1-\frac{1}{2 !}\left(x-x_{0}\right)^{2}+\frac{1}{4 !}\left(x-x_{0}\right)^{4}=\frac{P^{2}-2 x^{2}}{P^{2}}$

where

$x_{0}=2 n \pi$

$x_{0}-\frac{\pi}{2} \leq x \leq x_{0} \leq \frac{P}{\sqrt{2}}$

and

$-1+\frac{1}{2 !}\left(x-x_{0}\right)^{2}-\frac{1}{4 !}\left(x-x_{0}\right)^{4}=\frac{P^{2}-2 x^{2}}{P^{2}}$

where

$x_{0}=(2 n+1) \pi$

$\frac{P}{\sqrt{2}} \leq x_{0} \leq x \leq x_{0}+\frac{\pi}{2}$

Introduce new variable $t=x-x_{0}$, then above equations are:

even modes

$t^{4}+4 !\left(\frac{2}{P^{2}}-\frac{1}{2}\right) t^{2}+4 ! \frac{4}{P^{2}} x_{0} t+4 ! \frac{2}{P^{2}} x_{0}^{2}=0$

where

$x_{0}=(2 n+1) \pi$

$-\frac{\pi}{2} \leq t \leq 0 \leq \frac{P}{\sqrt{2}}-x_{0}$

and

$t^{4}-4 !\left(\frac{2}{P^{2}}+\frac{1}{2}\right) t^{2}-4 ! \frac{4}{P^{2}} x_{0} t+4 ! \cdot 2\left(1-\frac{x_{0}^{2}}{P^{2}}\right)=0$

where

$x_{0}=2 n \pi$ 
$\frac{P}{\sqrt{2}}-x_{0} \leq 0 \leq t \leq \frac{\pi}{2}$

\section{odd modes}

$t^{4}+4 !\left(\frac{2}{P^{2}}-\frac{1}{2}\right) t^{2}+4 ! \frac{4}{P^{2}} x_{0} t+4 ! \frac{2}{P^{2}} x_{0}^{2}=0$

where

$$
\begin{aligned}
& x_{0}=2 n \pi \\
& -\frac{\pi}{2} \leq t \leq 0 \leq \frac{P}{\sqrt{2}}-x_{0}
\end{aligned}
$$

and

$t^{4}-4 !\left(\frac{2}{P^{2}}+\frac{1}{2}\right) t^{2}-4 ! \frac{4}{P^{2}} x_{0} t+4 ! \cdot 2\left(1-\frac{x_{0}^{2}}{P^{2}}\right)=0$

where

$$
\begin{aligned}
& x_{0}=(2 n+1) \pi \\
& \frac{P}{\sqrt{2}}-x_{0} \leq 0 \leq t \leq \frac{\pi}{2}
\end{aligned}
$$

For each equation (D-26), (D-29), (D-32), (D2d) use formulas (E-20) - (E-23) form Appendix $\mathrm{E}$ to find roots $t_{1}, t_{2}, t_{3}, t_{4}$. Then actual root has to be chosen from $t_{1}, t_{2}, t_{3}, t_{4}$ which lays in the interval:

for (D-26), (D-32) $\quad-\frac{\pi}{2} \leq t \leq 0$

for (D-29), (D-35) $0 \leq t \leq \frac{\pi}{2}$

Modal effective index then is found from,

$$
n_{e f f}=\sqrt{n_{c}^{2}-\left(\frac{t_{i}+x_{0}}{k \mathrm{v}}\right)^{2}}
$$




\section{Appendix E}

\section{Quartic equation}

General form of quartic equation has five terms [53]:

$z^{4}+a_{3} z^{3}+a_{2} z^{2}+a_{1} z+a_{0}=0$

Its solutions are:

$z_{1}=-\frac{1}{4} a_{3}+\frac{1}{2} R+\frac{1}{2} D$

$z_{2}=-\frac{1}{4} a_{3}+\frac{1}{2} R-\frac{1}{2} D$

$z_{3}=-\frac{1}{4} a_{3}-\frac{1}{2} R+\frac{1}{2} E$

$z_{4}=-\frac{1}{4} a_{3}-\frac{1}{2} R-\frac{1}{2} E$

where,

$$
\begin{aligned}
& R=\sqrt{\frac{1}{4} a_{3}^{2}-a_{2}+y_{1}} \\
& D=\left\{\begin{array}{ccc}
\sqrt{\frac{3}{4} a_{3}^{2}-R^{2}-2 a_{2}+\frac{4 a_{3} a_{2}-8 a_{1}-a_{3}^{3}}{4 R}} & \text { for } & R \neq 0 \\
\sqrt{\frac{3}{4} a_{3}^{2}-2 a_{2}+2 \sqrt{y_{1}^{2}-4 a_{0}}} & \text { for } & R=0
\end{array}\right. \\
& E=\left\{\begin{array}{ccc}
\sqrt{\frac{3}{4} a_{3}^{2}-R^{2}-2 a_{2}-\frac{4 a_{3} a_{2}-8 a_{1}-a_{3}^{3}}{4 R}} & \text { for } & R \neq 0 \\
\sqrt{\frac{3}{4} a_{3}^{2}-2 a_{2}-2 \sqrt{y_{1}^{2}-4 a_{0}}} & \text { for } R=0
\end{array}\right.
\end{aligned}
$$

$y_{1}$ is a root of cubic equation (E-9),

$y^{3}-a_{2} y^{2}+\left(a_{1} a_{3}-4 a_{0}\right) y+\left(4 a_{2} a_{0}-a_{1}^{2}-a_{3}^{2} a_{0}\right)=0$ 
any root of (E-9) will work, i.e. any root of (E-9) will generate the same set $\left\{z_{1}, z_{2}, z_{3}, z_{4}\right\}$, therefore we can chose for instance the real root.

Roots $y_{1}, y_{2}, y_{3}$ of general cubic equation (E-10) are,

$$
\begin{aligned}
& y^{3}+A_{2} y^{2}+A_{1} y+A_{0}=0 \\
& y_{1}=-\frac{1}{3} A_{2}+(S+T) \\
& y_{2}=-\frac{1}{3} A_{2}-\frac{1}{2}(S+T)+j \frac{1}{2} \sqrt{3}(S-T) \\
& y_{3}=-\frac{1}{3} A_{2}-\frac{1}{2}(S+T)-j \frac{1}{2} \sqrt{3}(S-T)
\end{aligned}
$$

where,

$$
\begin{aligned}
& S=\sqrt[3]{P+\sqrt{B}} \\
& T=\sqrt[3]{P-\sqrt{B}} \\
& B=Q^{3}+P^{2} \\
& Q=\frac{3 A_{1}-A_{2}^{2}}{9} \\
& P=\frac{9 A_{2} A_{1}-27 A_{0}-2 A_{2}^{3}}{54}
\end{aligned}
$$

In order to use general solutions (E-2) - (E-5) we have to check the condition $R=0$.

Our quartic equations in Appendix D are with coefficients $a_{3}=0$ and $a_{1} \neq 0$. Such special form of equation, (E-19) has solutions (E-20) - (E-23), which do not require to test for condition $R=0$.

Special form of quartic equation [54]

$$
\begin{aligned}
& z^{4}+a_{2} z^{2}+a_{1} z+a_{0}=0 \\
& z_{1}=\frac{1}{2}\left(+\sqrt{a_{2}+2 u}+\sqrt{-\left(3 a_{2}+2 u+\frac{2 a_{1}}{\sqrt{a_{2}+2 u}}\right)}\right)
\end{aligned}
$$


$z_{2}=\frac{1}{2}\left(+\sqrt{a_{2}+2 u}-\sqrt{-\left(3 a_{2}+2 u+\frac{2 a_{1}}{\sqrt{a_{2}+2 u}}\right)}\right)$

$z_{3}=\frac{1}{2}\left(-\sqrt{a_{2}+2 u}+\sqrt{-\left(3 a_{2}+2 u-\frac{2 a_{1}}{\sqrt{a_{2}+2 u}}\right)}\right)$

$z_{4}=\frac{1}{2}\left(-\sqrt{a_{2}+2 u}-\sqrt{-\left(3 a_{2}+2 u-\frac{2 a_{1}}{\sqrt{a_{2}+2 u}}\right)}\right)$

where,

$u=-\frac{5}{6} a_{2}+\frac{P}{3 \cdot \sqrt[3]{R}}-\sqrt[3]{R}$

$P=-\frac{a_{2}^{2}}{12}-a_{0}$

$Q=-\frac{a_{2}^{3}}{108}+\frac{a_{2} a_{0}}{3}-\frac{a_{1}^{2}}{8}$

$R=\frac{Q}{2}+\sqrt{\frac{Q^{2}}{4}+\frac{P^{3}}{27}}$

When roots are real, then $z_{1}$ is always a positive root, $z_{4}$ is always a negative root, roots $z_{2}, z_{3}$ might be positive or negative depending on coefficients $a_{2}, a_{1}, a_{0}$. 


\section{Appendix $F$ \\ Optical Kerr effect. (followed by [23], [24], [25])}

When light propagates in the media electromagnetic field acting on the electrons of that media. This results in induced polarization. Normally field which keeps electrons near the atoms are larger than electromagnetic field of propagating light. Then induced polarization is just small perturbation linearly proportional to the field. However, when propagating light is intense, both competing fields can be of comparable magnitude. Higher order terms in the induced polarization can not be ignored any more.

$P=\varepsilon_{0} \chi^{(1)} E+\varepsilon_{0} \chi^{(2)} E^{2}+\varepsilon_{0} \chi^{(3)} E^{3}+\ldots$

where $\chi^{(1)}$ is linear susceptibility, $\chi^{(2)}, \chi^{(3)} \ldots$ are the nonlinear susceptibilities. For anisotropic media (crystal) $\chi^{(1)}, \chi^{(2)}, \chi^{(3)} \ldots$ are tensor quantities, otherwise (for fused silica as in optical fiber) they are scalars. For media, which is symmetric with respect to certain point ( point called center of inversion and media called centrosymmetric), $\chi^{(n)}=0$ for all even numbers $n$. Then, up to the third order of nonlinear effects polarization is,

$$
\begin{aligned}
& P \approx \varepsilon_{0} \chi^{(1)} E+\varepsilon_{0} \chi^{(3)} E^{3} \\
& E=E_{0} \cdot \cos (\omega t) \\
& P \approx \varepsilon_{0} \chi^{(1)} E+\varepsilon_{0} \chi^{(3)} E^{3}=\varepsilon_{0} \chi^{(1)} E_{0} \cos (\omega t)+\varepsilon_{0} \chi^{(3)} E_{0}{ }^{3} \cos ^{3}(\omega t) \\
& \cos ^{3}(\omega t)=\frac{1}{4} \cos (3 \omega t)+\frac{3}{4} \cos (\omega t) \\
& P_{\omega} \approx \varepsilon_{0} \chi_{\omega}^{(1)} E_{0} \cos (\omega t)+\frac{3}{4} \varepsilon_{0} \chi_{\omega}^{(3)} E_{0}{ }^{3} \cos (\omega t)=\varepsilon_{0}\left[\chi_{\omega}^{(1)}+\frac{3}{4} \chi_{\omega}^{(3)}\left|E_{0}\right|^{2}\right] E
\end{aligned}
$$

here $P_{\omega}$ is polarization response at frequency $\omega$ only, we do not consider polarization response at frequency $3 \omega$.

$$
P_{\omega}=\varepsilon_{0}\left[\varepsilon_{r}-1\right] E
$$


Compare (F-6) and (F-7) we find,

$\varepsilon_{r}=1+\chi_{\omega}^{(1)}+\frac{3}{4} \chi_{\omega}^{(3)}\left|E_{0}\right|^{2}$

Effective refractive index, $n_{\text {eff }}$ is:

$$
\begin{aligned}
& n_{e f f}=\sqrt{\varepsilon_{r}}=\sqrt{1+\chi_{\omega}^{(1)}+\frac{3}{4} \chi_{\omega}^{(3)}\left|E_{0}\right|^{2}}=\sqrt{1+\chi_{\omega}^{(1)}} \cdot \sqrt{1+\frac{3}{4} \chi_{\omega}^{(3)}\left|E_{0}\right|^{2} \frac{1}{1+\chi_{\omega}^{(1)}}} \\
& \approx \sqrt{1+\chi_{\omega}^{(1)}} \cdot\left[1+\frac{1}{2} \cdot \frac{3}{4} \chi_{\omega}^{(3)}\left|E_{0}\right|^{2} \frac{1}{1+\chi_{\omega}^{(1)}}\right]=\sqrt{1+\chi_{\omega}^{(1)}}+\frac{1}{2} \cdot \frac{3}{4} \chi_{\omega}^{(3)}\left|E_{0}\right|^{2} \frac{1}{\sqrt{1+\chi_{\omega}^{(1)}}}
\end{aligned}
$$

we have taken into account that

$$
1>>\frac{3}{4} \chi_{\omega}^{(3)}\left|E_{0}\right|^{2} \frac{1}{1+\chi_{\omega}^{(1)}}
$$

therefore:

$$
n_{\text {eff }} \approx n_{0}+n_{2}\left|E_{0}\right|^{2}
$$

the last expression is known as the optical Kerr effect, where

$n_{0}=\sqrt{1+\chi_{\omega}^{(1)}}$ is linear (low intensity) component of refractive index, $n_{2}=\frac{3}{8} \chi_{\omega}^{(3)} \frac{1}{n_{0}}$ is Kerr coefficient. 


\section{Bibliography}

[1] "Long-period fiber gratings as band-rejection filters", A.M. Vengsarkar et al, $J$. Lightwave Technol., vol. 14, pp. 58-65, 1996.

[2] "Band-rejection fibre filter using periodic core deformation", C. Narayanan et al, Electron. Lett., vol. 33, pp. 280-281, 1997.

[3] "All-Fiber Bandpass Filter with Adjustable Transmission Using Cladding-Mode Coupling", D.S. Starodubov et al, IEEE Photonics Technol. Lett., vol. 10, pp. 1590$1592,1998$.

[4] "Long period copper-coated grating as an electrically tunable wavelength-selective filter", O. Duhem et al, Electron. Lett., vol. 35, pp. 1014-1016, 1999.

[5] "Multi-stage Erbium-doped Fiber Amplifier Designs", J.M.P. Delavaux et al, $J$. Lightwave Technol., vol. 13, pp. 703-720, 1995.

[6] "A Low-Noise and Gain-Flattened Amplifier Composed of a Silica-Based and a FluorideBased $\mathrm{Er}^{3+}$-Doped Fiber Amplifier in a Cascade Configuration ", M. Yamada et a1, Electron. Lett., vol. 8, pp. 620-622, 1996.

[7] "Tailoring long period optical fibre gratings for flattening EDFA gain spectra", P. D. Greene et al, Electron. Lett., vol. 35, pp. 1373-1374, 1999.

[8] "Phase shifted and cascaded long-period fiber gratings", Y. Liu et al, Opt. Communic., vol. 164, pp. 27-31, 1999. 
[9] "EDFA gain flattening using phase-shifted long-period grating", Y. Zhu et al, Micrrowave Opt. Technol Lett., vol. 37, pp. 153-157, 2003.

[10] "Gain flattening by long period gratings in erbium doped fibers", R. Singth et al, Opt. Communic., vol. 240, pp. 123-132, 2004.

[11] "Laser-to-Fiber Coupling Scheme by Utilizing a Lensed Fiber Integrated with a LongPeriod Fiber Grating”, W.T. Chen et al, IEEE Photonics Technol. Lett., vol. 12, pp. $501-503,2000$.

[12] "Wavelength-selective coupler and add-drop multiplexer using long-period fiber gratings", V. Grubsky et al, Optical Fiber Communication Conference, 2000, 7-10 March 2000, vol. 4, pp. 28-30, 2000.

[13] "Dispersion control with use of long-period fiber gratings", D.B. Stegall et al, J. Opt. Soc. Am. A., vol. 17, pp. 304-312, 2000.

[14] "Optical fibre long-period grating sensors: characteristics and application", S.W. James et al, Mes. Sci. Technol., vol. 14, pp. R49-R61, 2003.

[15] "Applications of long-period gratings to single and multi-parameter sensing", V. Bhatia, Opt. Express., vol. 4, pp. 457-466, 1999.

[16] "Temperature-insensitive and strain insensitive long-period grating sensors for smart structures", V. Bhatia, Opt. Eng., vol. 36, pp. 1872-1876, 1997.

[17] "Long-period gratings in planar optical waveguides", V. Rastogi, et al, Appl. Opt., vol. 41, pp. 6351-6355, 2002.

[18] "Analysis of Corrugated Long-Period Gratings in Slab Waveguides and Their Polarization Dependence", Q. Liu, et al, J. Lightwave Technol., vol. 21, pp. 3399$3405,2003$. 
[19] "Widely Tunable Long-Period Gratings Fabricated in Polymer-Clad Ion-Exchanged Glass Waveguides", K.S. Chiang, et al, IEEE Photonics Technol. Lett., vol. 15, pp. 1094-1096, 2003.

[20] "Long-Period Waveguide Gratings", K.S. Chiang et al, Japanese Journal of Applied Physics, vol. 43, pp. 5690-5696, 2004.

[21] "Tunable notch filter using a thermo-optic long-period grating,", M.S. Kwon et al, $J$. Lightwave Technol., vol. 22, pp. 1968-1975, 2004.

[22] "Characteristics of Polymer Waveguide Notch Filters Using Thermooptic Long-Period Gratings", M.S. Kwon et al, IEEE Journal of Selected Topics in Quantum Electronics, vol. 11, pp. 190-196, 2005.

[23] "The Elements of Nonlinear Optics", A.M. Butcher, D.Cotter, Cambridge University Press, sec. 6.3, 1990.

[24] "Optical Communication Systems", J. Gowar, Prentice Hall, second edition, sec. 6.3, 1993.

[25] "Resonantly Enhanced Nonlinearity in Doped Fibers for Low-Power All-Optical Switching: A Review", M.J.F. Digonnet et al, Opt. Fiber Technol., vol. 3, pp. 44-64, 1997.

[26] "Analysis of a widely tunable long-period grating by use of an ultrathin cladding layer and higher-order cladding mode coupling", K.W. Chung et al, Opt. Lett., vol. 29, pp. 812-814, 2004.

[27] "Analysis of optical response of long period fiber gratings to nm-thick thin-film coatings", Z. Wang et al, Opt. Express, vol. 13, pp. 2808-2813, 2005.

[28] "Optical Waveguide Theory", A.M. Snider and J.D. Love, Chapman \& Hall, 1983. 
[29] "Theory of Dielectric Optical Waveguide", D. Marcuse, Academic Press, 1991.

[30] "Optical Waves in Crystals", A. Yariv and P. Yeh, John Wiley \& Sons, 2003.

[31] "Fiber Grating Spectra", T. Erdogan, J. Lightwave Technol., vol. 15, pp. 1277-1294, 1997.

[32] "Microwave Engineering”, D.M. Pozar, Addison-Wesley, 1990.

[33] "An Introduction to Optical Waveguides", M.J. Adams, John Wiley \& Sons, 1981.

[34] “Optical Waveguides”, N.S. Kapany, J.J. Burke, Academic Press, 1972.

[35] "Characteristics of five-layer planar dielectric waveguides", A.S. Belanov, Sov. J. Quantum Electron., vol. 7, pp. 219-222, 1977.

[36] "General formulas for the guiding properties of a multilayer slab waveguide", Y.F. Li et al, J. Opt. Soc. Am. A., vol. 4, pp. 671-677, 1987.

[37] "Guiding properties of multilayer slab waveguides", J.W.Y. Lit et al, Can. J. Phys., vol. 66, pp. 914-940, 1988.

[38] "Characteristics of Optical Guided Modes in Multilayer Metal-Clad Planar Optical Guide with Low-Index Dielectric Buffer Layer", Y. Yamamoto et al, IEEE J. Quantum Electron., vol. QE-11, pp. 729-736, 1975.

[39] "Dual resonant peaks of $\mathrm{LP}_{015}$ cladding mode in long-period gratings", X. Shu et al, Electron. Lett., vol. 35, pp. 649-651, 1999.

[40] "Numerical Recipes", W.H. Press et al, Cambridge University Press, vol. 1, ch. 10, 1992.

[41] "Theory and practice of long-period gratings: when a loss becomes a gain", X. Daxhelet et al, Opt. Lett., vol. 28, pp. 686-688, 2003. 
[42] "Optimization of sensitivity in Long Period Fiber Gratings with overlay deposition", I.D. Villar et al, Opt. Express., vol. 13, pp. 56-69, 2005.

[43] "Tunable Optical Filters for Dense WDM Networks", D. Sadot et al, IEEE Communication Magazine, December, pp. 50-55, 1998.

[44] "Bandpass filters based on $\pi$-shifted long period fiber gratings for actively mode-locked erbium fiber lasers", O. Deparis et al, Opt. Lett., vol. 26, pp. 1239-1241, 2001.

[45] "Reconfigurable $\pi$-Shifted and Mach-Zehnder Bandpass Filters on the Basis of Electrooptically Induced Long-Period Gratings in a Planar Waveguide", M. Kulishov et al, J. Lightwave Technol., vol. 21, pp. 854-861, 2003.

[46] "Analysis of the effective-index method for the vector modes of rectangular-core dielectric waveguides", K.S. Chiang, IEEE Trans. Microwave Theory Tech., vol. 44, pp. 692-700, 1996.

[47] "Theory and practice of long-period gratings: when a loss becomes a gain", X. Daxhelet et al, Opt. Lett., vol. 28, pp. 686-688, 2003.

[48] "Irreversible coupling by use of dissipative optics", M. Greenberg et al, Opt. Lett., vol. 29, pp. 451-453, 2004.

[49] "Unified approach for calculating the number of confined modes in multilayered waveguiding structures", S. Ruschin et al, J. Opt. Soc. Am. A., vol. 3, pp. 116-123, 1986.

[50] "Quantum mechanics", C. Cohen-Tannoudyi, John Wiley \& Sons, vol. 2, 1977.

[51] "Quantum theory”, D. Bohm, Dover Publications, 1979.

[52] "Mathematical analysis of physical problems", P.R. Wallace, Dover Publications, 1984.

[53] "CRC Concise Encyclopedia of Mathematics", E.W. Weisstein, CRC Press, 1999. 
[54] http://www.algebra.com/algebra/about/history/Quartic equation.wikipedia

[55] "Resonance Peak Shift and Dual Peak Separation of Long-Period Fiber Gratings for Sensing Applications", Y.G. Han et al, IEEE Photonics Technol. Lett., vol. 13, pp. 699-701, 2001.

[56] "Wave-particle duality", Franco Selleri, Plenum Press, 1992.

[57] "Double-ion-exchange process in glass for the fabrication of computer-generated waveguide holograms", J. Saarinen et al, Appl. Opt., vol. 33, pp. 3353-3359, 1994.

[58] "Propagation loss characteristics of long silica-based optical waveguides on 5 inch $\mathrm{Si}$ wafers", Y. Hibino et al, Electron. Lett., vol. 29, pp. 1847-1848, 1993. 